\title{
[4+2]-Annulations of Aminocyclobutanes.
}

\author{
Daniele Perrotta, Sophie Racine, Jeremy Vuilleumier, Florian de Nanteuil and Jerome Waser.* ${ }^{\dagger}$ \\ ${ }^{\dagger}$ Laboratory of Catalysis and Organic Synthesis, Ecole Polytechnique Fédérale de Lausanne, EPFL SB ISIC LCSO, BCH \\ 4306, 1015 Lausanne, Switzerland.
}

Supporting Information Placeholder

\begin{abstract}
The first [4+2]-annulation between aminocyclobutanes and aldehydes to access tetrahydropyranyl amines is reported. With phthalimido cyclobutane dicarboxylates and aromatic aldehydes, tetrahydropyrans were obtained in 53-92\% yield and 3:117:1 dr using scandium triflate or iron trichloride as catalyst. The use of thymine- or fluorouracil- substituted cyclobutanes gave direct access to six-membered ring nucleoside analogues. Finally, the [4+2]-annulation between aminocyclobutanes and enol ethers led to the corresponding cyclohexylamines.
\end{abstract}

Six-membered nitrogen-substituted carbo- and heterocycles are among the most frequently encountered scaffolds in natural and synthetic bioactive compounds (Figure 1). A cyclohexylamine or a tetrahydropyranylamine ring for example can be found in the core of the natural alkaloids strychnine (1) and staurosporine (2) respectively. The synthetic antiviral drug Tamiflu (3) is constituted by a cyclohexenyldiamine core. Synthetic methods giving access to these important scaffolds with high efficiency and broad scope are desirable to accelerate the discovery of new bioactive compounds. Whereas the Diels-Alder reaction has emerged as a powerful method to synthesize cyclohexenylamines and dihydropyranylamines, ${ }^{1}$ there is currently a lack of transformations giving straightforward access to saturated ring systems with high convergence.

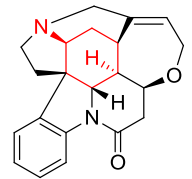

Strychnine, 1 (CNS stimulator)

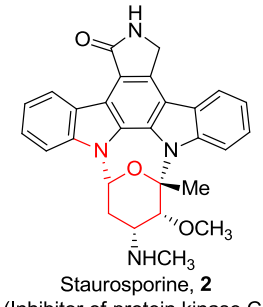

(Inhibitor of protein kinase C)

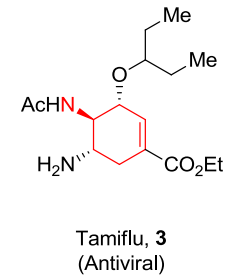

Figure 1. Bioactive compounds containing nitrogensubstituted six-membered rings.

The use of annulation reactions of donor-acceptor substituted strained rings constitutes a valuable alternative for the synthesis of saturated carbo- or heterocycles. In the case of sixmembered rings, the [4+2]-annulation between donor-acceptor cyclobutanes and olefins or carbonyl compounds appears particularly attractive (Scheme 1, A). Nevertheless, the chemistry of donor-acceptor cyclobutanes has been much less developed than for the corresponding cyclopropanes. ${ }^{2}$ It is only very recently that more general catalytic methods have been developed in the groups of Johnson, Christie and Pritchard, and Pagenkopf in particular (Scheme $1, \mathbf{B}) .{ }^{3}$ However, these works focused on the use of oxygen and carbon as electron-donating groups, and the scope of substituents on the cyclobutanes was often limited. In the case of nitrogen as donor, an important pioneering result has been reported by Saigo and co-workers in $1991 .^{4}$ Unfortunately, the precious nitrogen functionality could not be conserved in the final product, as hydrolysis occurred upon work-up.

Scheme 1. [4+2]-Annulations for the Synthesis of SixMembered Rings.

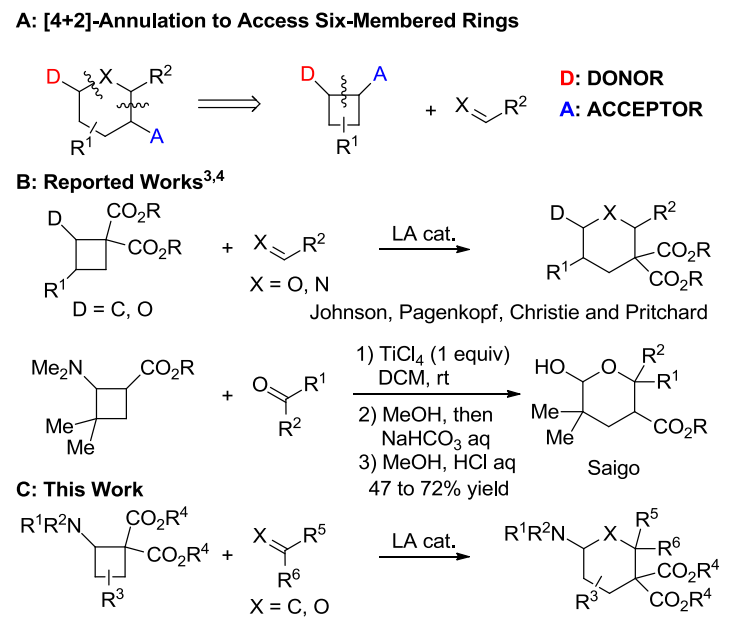

Recognizing the underexploited potential of nitrogensubstituted strained rings for the synthesis of bioactive compounds, ${ }^{5}$ our group has initially focused on the discovery of new types of donor-acceptor systems which could be broadly applied in annulation reactions. In particular, we reported that imido-substituted cyclopropane dicarboxylates can be used in $[3+2]$-annulations with both enol ethers and carbonyl compounds under mild catalytic conditions. ${ }^{6}$ In 2013 , we reported a new method to access the corresponding imido substituted cyclobutane dicarboxylates. ${ }^{7}$ A single example of [4+2]annulation between an aminocyclobutane and an enol ether 
was also described in this work. Herein, we report the first Lewis acid-catalyzed [4+2]-annulation reaction between donor-acceptor aminocyclobutanes and carbonyl compounds and a further extended scope of the reaction with enol ethers (Scheme 1, C). In the case of aldehydes, the reaction was also successful for multi-substituted aminocyclobutanes, leading to tetrahydrofurylamines bearing up to three distinct stereocenters.

We stared our investigations by examining the [4+2]annulation of aminocyclobutane $\mathbf{4 a},{ }^{7}$ and benzaldehyde $\mathbf{5 a}$ (Table 1). The reaction proceeded with $20 \mathrm{~mol} \% \mathrm{FeCl}_{3} \bullet \mathrm{Al}_{2} \mathrm{O}_{3}$ as catalyst, which had been used in the corresponding reaction with aminocyclopropanes, ${ }^{6 c}$ but the product was obtained only with low diastereoselectivity as part of a complex mixture (Table 1, entry 1). A higher diastereoselectivity (6:1) was observed with tin tetrachloride, previously used for the single example reported of [4+2]-annulation of enol ethers and aminocyclobutanes (Table 1 , entry 2$)^{7}$ However, a complex mixture was also observed in this case. As the use of titanium tetrachloride did not lead to any improvement (Table 1, entry 3), we then turned to well-established metal triflates as catalysts. Whereas no reaction was observed with ytterbium triflate (Table 1, entry 4) and a complex mixture was obtained with hafnium triflate (Table 1, entry 5), both indium and scandium triflates led to complete conversion without the formation of side products (Table 1, entries 6 and 7). A better diastereoselectivity (13:1 vs 9:1) was observed in the case of scandium triflate.

Table 1. Optimization of the [4+2]-Annulation.

\begin{tabular}{|c|c|c|c|c|c|}
\hline entry & 4, R & catalyst & time & conversion $^{\mathrm{a}}$ & $\mathrm{dr}^{\mathrm{b}}$ \\
\hline 1 & $\mathbf{4 a}, \mathrm{H}$ & $\mathrm{FeCl}_{3} \bullet \mathrm{Al}_{2} \mathrm{O}_{3}$ & $40 \mathrm{~min}$ & $>95 \%{ }^{\mathrm{c}}$ & $2: 1$ \\
\hline 2 & 4a, $\mathrm{H}$ & $\mathrm{SnCl}_{4}$ & $2.5 \mathrm{~h}$ & $>95 \%^{\mathrm{c}}$ & $6: 1$ \\
\hline 3 & 4a, $\mathrm{H}$ & $\mathrm{TiCl}_{4}$ & $2.5 \mathrm{~h}$ & $88 \%^{\mathrm{c}}$ & $5: 1$ \\
\hline 4 & 4a, $H$ & $\mathrm{Yb}(\mathrm{OTf})_{3}$ & $2.5 \mathrm{~h}$ & $<5 \%{ }^{\mathrm{d}}$ & - \\
\hline 5 & $\mathbf{4 a}, \mathrm{H}$ & $\mathrm{Hf}(\mathrm{OTf})_{4}$ & $2.5 \mathrm{~h}$ & $>95 \%^{\mathrm{c}}$ & $9: 1$ \\
\hline 6 & $4 \mathbf{a}, \mathrm{H}$ & $\operatorname{In}(\mathrm{OTf})_{3}$ & $2.5 \mathrm{~h}$ & $>95 \%$ & $9: 1$ \\
\hline 7 & $\mathbf{4 a}, \mathrm{H}$ & $\mathrm{Sc}(\mathrm{OTf})_{3}$ & $2.5 \mathrm{~h}$ & $>95 \%$ & $13: 1$ \\
\hline $8^{e}$ & $4 \mathrm{~b}, \mathrm{Me}$ & $\mathrm{Sc}(\mathrm{OTf})_{3}$ & $4 \mathrm{~h}$ & $<5 \%{ }^{\mathrm{d}}$ & - \\
\hline 9 & 4b, Me & $\mathrm{FeCl}_{3} \cdot \mathrm{Al}_{2} \mathrm{O}_{3}$ & $2.5 \mathrm{~h}$ & $57 \%$ & $1.5: 1$ \\
\hline 10 & $\mathbf{4 b}, \mathrm{Me}$ & $\mathrm{FeCl}_{3} \cdot \mathrm{Al}_{2} \mathrm{O}_{3}{ }^{\mathrm{f}}$ & $5 \mathrm{~h}$ & $>95 \%$ & $5: 1$ \\
\hline
\end{tabular}

${ }^{a}$ Reaction conditions: $0.05 \mathrm{mmol} \mathrm{4,} 0.075 \mathrm{mmol}$ 5a, $20 \mathrm{~mol} \%$ catalyst in $1.5 \mathrm{~mL} \mathrm{CH}_{2} \mathrm{Cl}_{2}$ at rt. Conversion estimated by the ratio of product 6 to cyclobutane 4 on the ${ }^{1} \mathrm{H}$ NMR of the crude mixture. ${ }^{b}$ Determined on the ${ }^{1} \mathrm{H}$ NMR of the crude mixture. ${ }^{\mathrm{c}}$ Complex mixture of products was observed by ${ }^{1} \mathrm{H}$ NMR. ${ }^{\mathrm{d}}$ No product observed. ${ }^{\mathrm{e}}$ Temperature increased from rt to $40{ }^{\circ} \mathrm{C}$ after $7 \mathrm{~h} .{ }^{\mathrm{f}} 100$ mol \% catalyst loading.

When the methyl-substituted aminocyclobutane $4 \mathbf{b}$ was examined, the reactivity dropped significantly and no conversion was observed with scandium triflate (Table 1, entry 8). However, product 6 ba could be obtained with $57 \%$ conversion and 1.5:1 dr when using $\mathrm{FeCl}_{3} \bullet \mathrm{Al}_{2} \mathrm{O}_{3}$ (Table 1, entry 9). Finally, increasing the amount of this cheap and non-toxic catalyst to
$100 \mathrm{~mol} \%$ allowed us to reach full conversion and 5:1 diastereoselectivity (Table 1 , entry 10). ${ }^{8}$

With optimized conditions in hand, we first examined the scope of the reaction of unsubstituted donor acceptor aminocyclobutanes (Table 2). On a preparative scale, tetrahydropyranyl amine 6aa could be isolated in 92\% yield and 16:1 dr in favor of the cis diastereoisomer (Table 2, entry 1). ${ }^{9}$ Electronwithdrawing and donating groups in para position of the aromatic ring were well tolerated (Table 2, entries 2 and 3), although a lower diastereoselectivity was observed in case of the methoxy substituent (Table 2, entry 3). Product 6ad bearing an ortho-methoxy substituent was obtained in 91\% yield and 9:1 dr (Table 2, entry 4). The [4+2]-annulation with cinnamaldehyde 5e proceeded in nearly quantitative yield, but with only 2:1 dr. Under these reaction conditions, only low yields $(<30 \%)$ and diastereoselectivities were obtained when using aliphatic aldehydes or ketones as partners (results not shown).

We then turned to the more challenging use of substituted aminocyclobutanes. With cyclobutane $\mathbf{4 b}$, the desired tetrahydropyranyl amine 6ba was obtained in 64\% yield as a 5:1 mixture of only two diastereoisomers at the benzylic center (Table 2 , entry 6 ). The main product obtained corresponded to the isomer with all substituents in equatorial position, which is probably the most stable. The reaction was also successful with para-chloro benzaldehyde as partner (Table 2, entry 7). When using phenyl-substituted cyclobutane $\mathbf{4 c}$, the reaction became slower. Nevertheless, the desired product 6ca could still be obtained in $53 \%$ yield (Table 2, entry 8 ). Finally, the annulation of cyclobutane $\mathbf{4 d}$ bearing a substituent in 3 position relative to the phthalimide was examined (Table 2, entries 9-10). This class of cyclobutanes can only be obtained as a mixture of diastereoisomers using our previously reported $[2+2]$-cycloaddition method. ${ }^{7}$ At room temperature, a significant amount of retro [2+2]-cycloaddition was observed, but this side reaction could be suppressed at $0{ }^{\circ} \mathrm{C}$. The desired products could then be obtained in $64-81 \%$ yield as a $3: 1 \mathrm{mix}-$ ture of diastereoisomers starting from a 1:1.1 mixture of cyclobutanes. The annulation reaction is therefore not stereospecific. Interestingly, we observed that the cis cyclobutane reacted faster than the trans isomer in the [4+2]-annulation. This difference in rate could be used to do a resolution of the difficult to separate isomers of aminocyclobutane $\mathbf{4 d}$ : with the less reactive indium triflate catalyst, the trans isomer of aminocyclobutane $\mathbf{1 b}$ could be recovered in quantitative yield and 14:1 dr (Scheme 2).

Scheme 2. Diastereospecific [4+2]-Annulation.

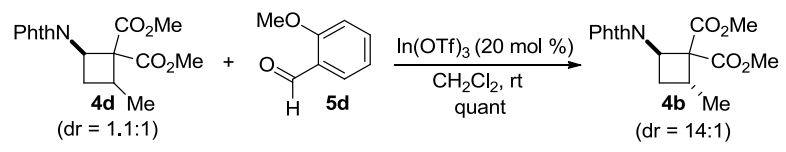

During the investigation of the scope of the [4+2]annulation reaction of unsubstituted aminocyclobutanes, a relatively high catalyst loading of scandium triflate (20 mol \%) has been used for practical reasons. Nevertheless, the catalyst loading could be decreased to $5 \mathrm{~mol} \%$ when the reaction was run on a $1 \mathrm{mmol}$ scale and product 3aa was obtained in $81 \%$ yield and 13:1 dr (Scheme 3). 
Scheme 3. [4+2]-Annulation at $1 \mathrm{mmol}$ Scale.

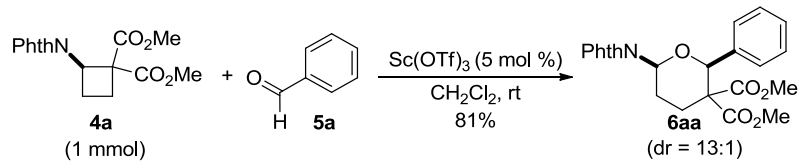

Table 2. Scope of the [4+2]-Annulation.

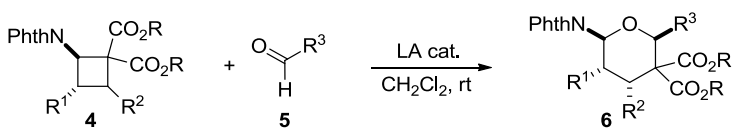

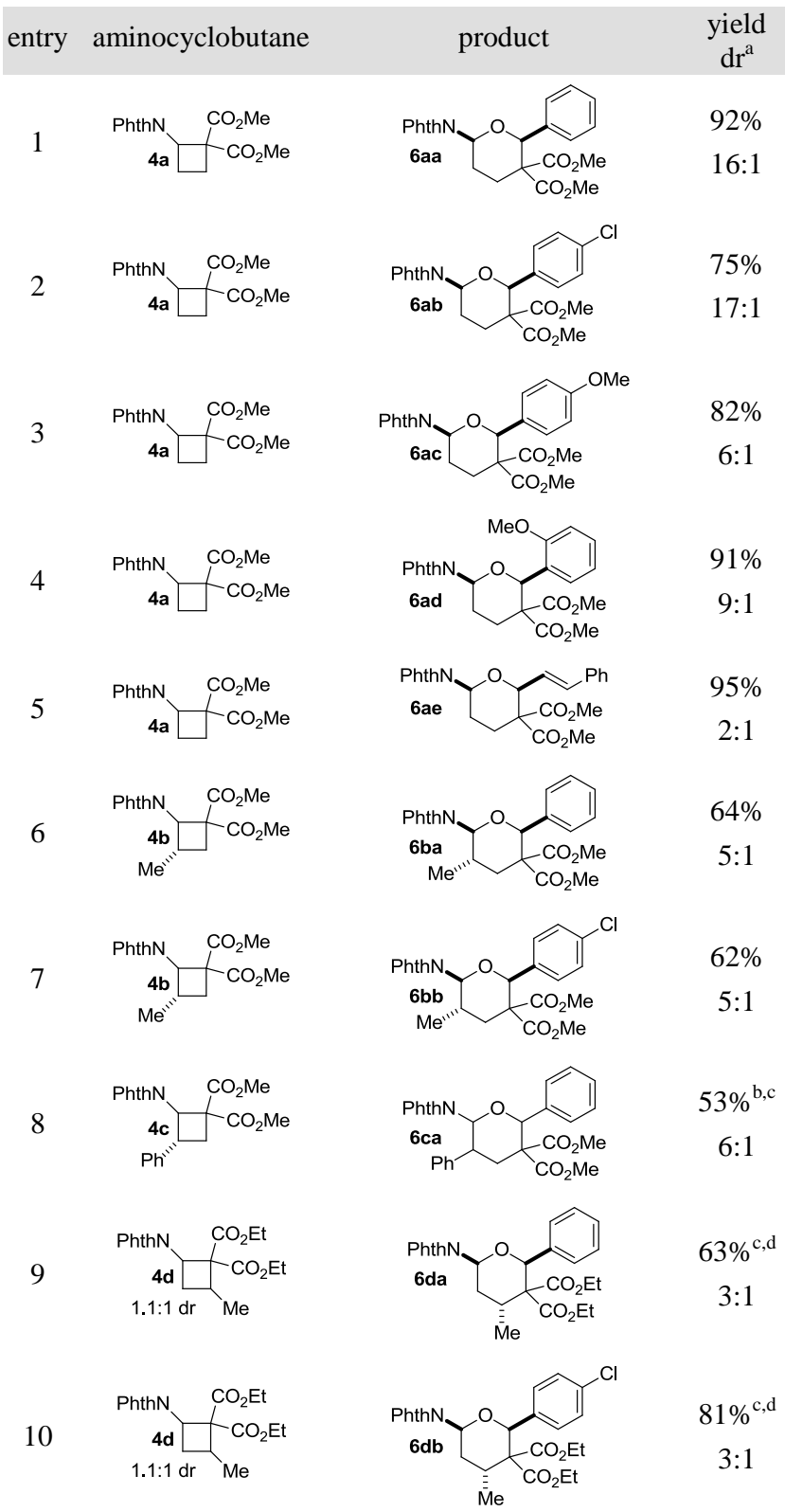

${ }^{a}$ Reaction conditions: With cyclobutane $\mathbf{4 a}$ : $0.20 \mathrm{mmol} \mathbf{4 a}, 0.30$ mmol 5, $0.040 \mathrm{mmol} \mathrm{Sc}(\mathrm{OTf})_{3}$ in $6 \mathrm{~mL} \mathrm{CH}_{2} \mathrm{Cl}_{2}, \mathrm{rt}, 2 \mathrm{~h}$. With cyclobutanes 4b-d: $0.20 \mathrm{mmol} \quad \mathbf{4}, 0.30 \mathrm{mmol}$ 5, $0.20 \mathrm{mmol}$ $\mathrm{FeCl}_{3} \cdot \mathrm{Al}_{2} \mathrm{O}_{3}$ in $6 \mathrm{~mL} \mathrm{CH} \mathrm{Cl}_{2}$, rt, $5 \mathrm{~h}$. Isolated yield after column chromatography are given. ${ }^{\mathrm{b}} 16 \mathrm{~h}$ reaction time. Structure not assigned due to peak overlap in ${ }^{1} \mathrm{H}$ NMR. ${ }^{c}$ Product obtained in $>90 \%$ purity by ${ }^{1} \mathrm{H}$ NMR, ${ }^{\mathrm{d}}$ Reaction mixture stirred for $2 \mathrm{~h}$ at 0 ${ }^{\circ} \mathrm{C}$.
Recently, our group has discovered that phthalimide could be replaced by a protected thymine or uracil derivative in donor-acceptor aminocyclopropanes to access nucleoside analogues. ${ }^{10}$ We wondered if this approach could also be applied to the [4+2]-annulation. In view of the synthesis of bioactive compounds containing a tetrahydropyran ring, this is particularly important, as the phthaloyl group cannot be removed on these substrates, in contrast to the corresponding carbocycles. ${ }^{6,7}$ Indeed, the reaction was successful with aminocyclobutane 4e bearing a benzoyl-protected thymine substituent (Scheme 4). In this case, best results were obtained using hafnium triflate as catalyst. ${ }^{8}$ As partial removal of the benzoyl group was observed during the reaction, the obtained crude mixture was directly treated with ammonium hydroxide in ethanol to obtain the fully deprotected product 6ea in $78 \%$ yield and 20:1 dr. ${ }^{11}$ The annulation with aminocyclobutane $\mathbf{4 e}$ was successful with aromatic aldehydes bearing an electronpoor or electron rich benzene ring or a thiophene heterocycle, giving the desired products 6ec-d and 6f-g in 70-79\% and 5:112:1 dr. Cinnamaldehyde 5e could also be used to give 6ee, although the diastereoselectivity was lower, as in the case of the phthalimide substituted cyclobutane. Finally, the [4+2]annulation also proceeded with benzoyl protected fluorouracil derivative $\mathbf{4 f}$ (product $\mathbf{6 f a}$ ).

Scheme 4. Nucleoside Analogues via [4+2]-Annulation.

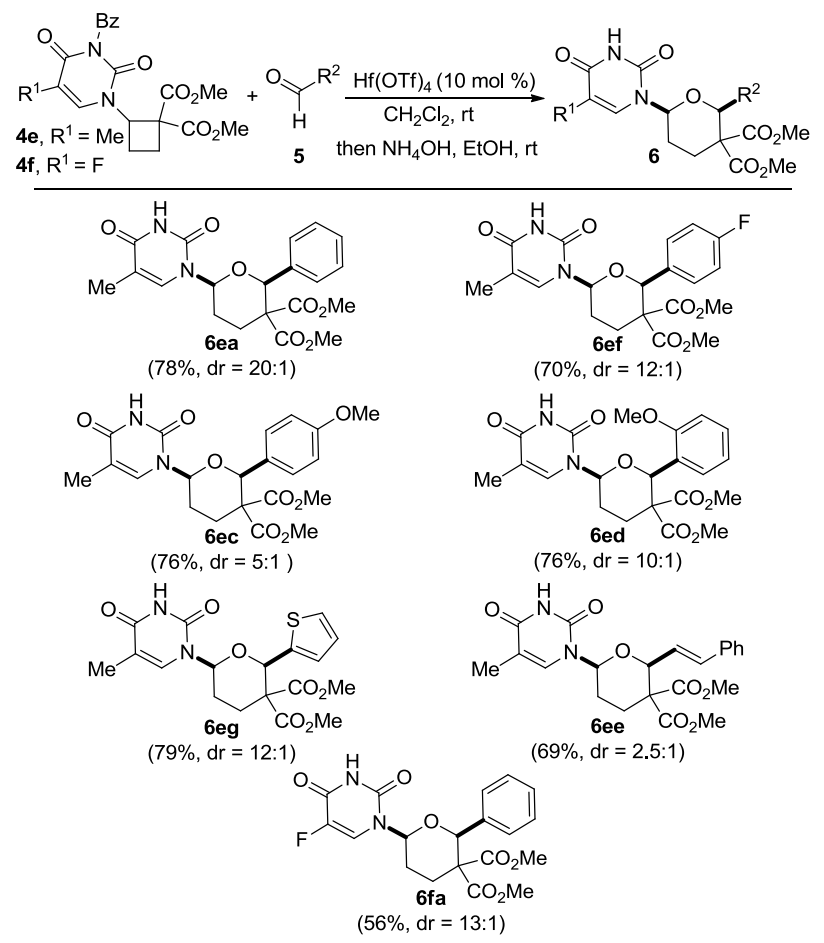

Reaction conditions: $0.30 \mathrm{mmol} \mathrm{4,} 0.45 \mathrm{mmol} \mathrm{5,} 0.030 \mathrm{mmol}$

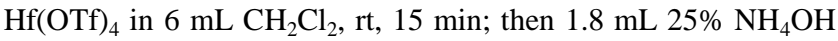
solution, $6 \mathrm{~mL} \mathrm{EtOH}$, rt, $16 \mathrm{~h}$. Isolated yield after column chromatography and crystallization are given.

Finally, we shortly examined the annulation reaction of enol ethers (Scheme 5). In this case, only tin tetrachloride at low temperature was successful as a catalyst. The reaction proceeded in high yield and diastereoselectivity with enol ethers substituted with a benzene derivative (products 8aa-c). However, the diastereoselectivity was lost when a more strongly electron-withdrawing group was present on the benzene ring 
or when a 1,2-disubstituted enol ether was used (products 8ad and 8ae). The latter result demonstrated that the [4+2]annulation is not stereospecific in relation to the geometry of the enol ether, in contrast to the previously reported [3+2]annulation reaction. ${ }^{6 \mathrm{a}}$ The higher sensitivity of the enol ethers in comparison to the aldehydes did not allow the use of substituted cyclobutanes in the annulation process.

Scheme 5. [4+2]-Annulation with Enol Ethers.

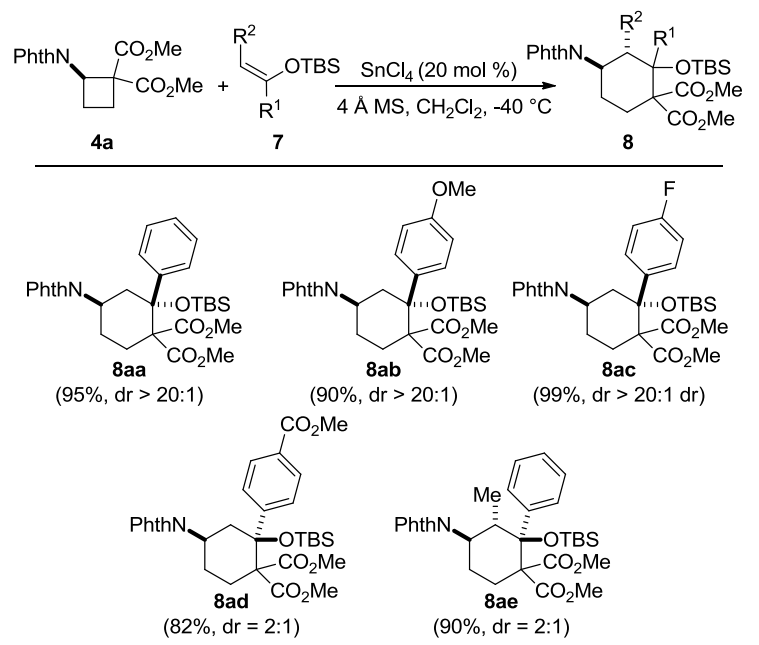

Reaction conditions: $0.20 \mathrm{mmol} \mathrm{4a}, 0.30 \mathrm{mmol}$ 7, $0.040 \mathrm{mmol}$ $\mathrm{SnCl}_{4}, 4 \AA \mathrm{MS}$ in $2 \mathrm{~mL} \mathrm{CH} \mathrm{Cl}_{2},-40{ }^{\circ} \mathrm{C}$. Isolated yields after column chromatography are given.

In conclusion, we have reported the first example of [4+2]annulation between aldehydes and donor-acceptor aminocyclobutanes which proceeds without loss of the precious nitrogen functionality. The reaction proceeded in high yield and diastereoselectivity with aromatic aldehydes when using unsubstituted phthalimido cyclobutane dicarboxylates. The annulation was also successful when introducing substituents in 1,2- or 1,3-positions to the phthalimide on the cyclobutane ring. The transformation could be as well applied to the synthesis of six-membered ring carbonucleoside analogues. The [4+2]-annulation involving enol ethers appears more limited at this stage, as only unsubstituted cyclobutanes could be used successfully. Overall, our work demonstrated that the polarization concepts used in the case of aminocyclopropanes can be extended to their one-carbon homologues. Nevertheless, the lower reactivity of the aminocyclobutanes makes the use of sensitive reaction partners and the control of stereoselectivity more challenging than for the corresponding cyclopropanes.

\section{ASSOCIATED CONTENT}

\section{Supporting Information}

Experimental procedures and analytical data for all new compounds. This material is available free of charge via the Internet at http://pubs.acs.org.

\section{AUTHOR INFORMATION}

\section{Corresponding Author}

* jerome.waser@epfl.ch

\section{ACKNOWLEDGMENT}

We thank F. Hoffmann-La Roche Ltd for an unrestricted research grant and the Swiss National Science Foundation (SNSF, grant numbers 200021_129874 and 200020_149494) and the NCCR chemical biology for financial support.

\section{REFERENCES}

(1) Selected examples: (a) Xu, Y.; Dolbier, W. R. J. Org. Chem 1997, 62, 6503. (b) Kozmin, S. A.; Janey, J. M.; Rawal, V. H. J. Org. Chem 1999, 64, 3039. (c) Huang, Y.; Rawal, V. H. Org. Lett. 2000, 2 , 3321. (d) Kozmin, S. A.; Iwama, T.; Huang, Y.; Rawal, V. H. J. Am. Chem. Soc. 2002, 124, 4628. (e) Xie, H.; Sammis, G. M.; Flamme, E. M.; Kraml, C. M.; Sorensen, E. J. Chem. Eur. J. 2011, 17, 11131.

(2) Selected reviews on donor-acceptor cyclopropanes: (a) Reissig, H. U.; Zimmer, R. Chem. Rev 2003, 103, 1151. (b) Yu, M.; Pagenkopf, B. L. Tetrahedron 2005, 61, 321. (c) De Simone, F.; Waser, J. Synthesis 2009, 3353. (d) Carson, C. A.; Kerr, M. A. Chem. Soc. Rev. 2009, 38, 3051. (e) Schneider, T. F.; Kaschel, J.; Werz, D. B. Angew. Chem., Int. Ed. 2014, 53, 5504.

(3) (a) Parsons, A. T.; Johnson, J. S. J. Am. Chem. Soc. 2009, 131, 14202. (b) Allart, E. A.; Christie, S. D. R.; Pritchard, G. J.; Elsegood, M. R. J. Chem. Commun. 2009, 7339. (c) Moustafa, M. M. A.; Pagenkopf, B. L. Org. Lett. 2010, 12, 4732. (d) Moustafa, M. M. A.; Stevens, A. C.; Machin, B. P.; Pagenkopf, B. L. Org. Lett. 2010, 12, 4736. (e) Stevens, A. C.; Palmer, C.; Pagenkopf, B. L. Org. Lett. 2011, 13, 1528. (f) Vemula, N.; Stevens, A. C.; Schon, T. B.; Pagenkopf, B. L. Chem. Commun. 2014, 50, 1668. (g) Okado, R.; Nowaki, A.; Matsuo, J.; Ishibashi, H. Chem. Pharm. Bull. 2012, 60, 21. (h) Matsuo, J.-I. Tetrahedron Lett. 2014, 55, 2589. (i) Souillart, L.; Cramer, N. Chem. Eur. J. 2015, 21, 1863.

(4) Shimada, S.; Saigo, K.; Nakamura, H.; Hasegawa, M. Chem. Lett. 1991, 1149.

(5) de Nanteuil, F.; De Simone, F.; Frei, R.; Benfatti, F.; Serrano, E.; Waser, J. Chem. Commun. 2014, 50, 10912.

(6) (a) de Nanteuil, F.; Waser, J. Angew. Chem., Int. Ed. 2011, 50, 12075. (b) Benfatti, F.; de Nanteuil, F.; Waser, J. Chem. Eur. J. 2012, 18, 4844. (c) Benfatti, F.; de Nanteuil, F.; Waser, J. Org. Lett. 2012, 14, 386. (d) de Nanteuil, F.; Loup, J.; Waser, J. Org. Lett. 2013, 15, 3738. (e) de Nanteuil, F.; Serrano, E.; Perrotta, D.; Waser, J. J. Am. Chem. Soc. 2014, 136, 6239. (f) Serrano, E.; de Nanteuil, F.; Waser, J. Synlett 2014, 25, 2285.

(7) de Nanteuil, F.; Waser, J. Angew. Chem., Int. Ed. 2013, 52, 9009.

(8) See the Supporting Information for a full list of tested reaction conditions and catalysts.

(9) The configuration of the tetrahydropyran products was assigned via 2D NMR experiments, see Supporting Information.

(10) Racine, S.; de Nanteuil, F.; Serrano, E.; Waser, J. Angew. Chem., Int. Ed. 2014, 53, 8484.

(11) After column chromatography, the obtained products still contained small amounts of benzamide originating from the protecting group. Further purification by crystallization allowed obtaining the pure nucleoside analogues. 
Table of Content Graphic

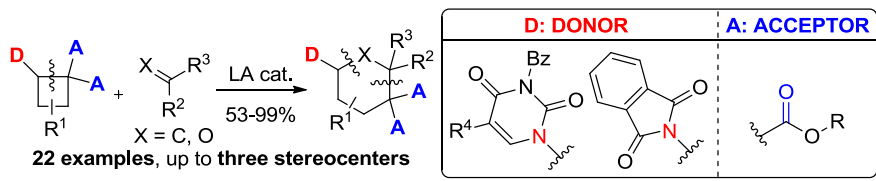




\section{Supporting Information}

\section{[4+2] Annulations of Aminocyclobutanes.}

\section{Daniele Perrotta, Sophie Racine, Jeremy Vuilleumier, Florian de Nanteuil and Jérôme Waser}

Laboratory of Catalysis and Organic Synthesis, Institute of Chemical Sciences and Engineering, Ecole Polytechnique Fédérale de Lausanne, EPFL SB ISIC LCSO, BCH 4306, 1015 Lausanne, Switzerland.

jerome.waser@epfl.ch

(89 pages)

\section{Table of Contents}

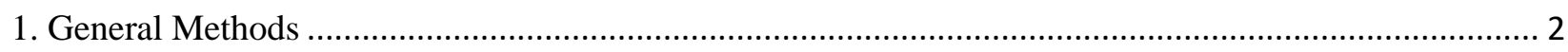

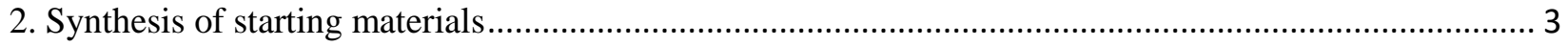

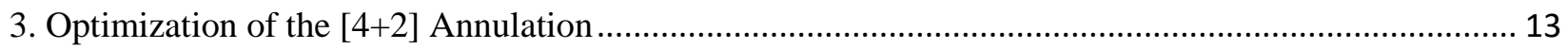

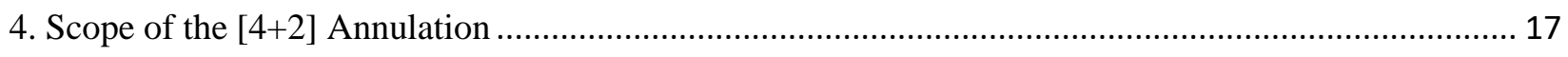

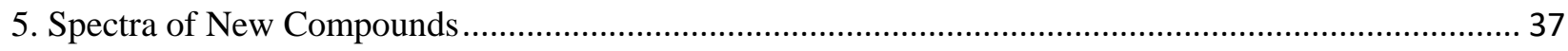




\section{General Methods}

All reactions were carried out in oven- or flame- dried glassware under an atmosphere of nitrogen, unless stated otherwise. For quantitative flash chromatography, distilled technical grade solvents were used. THF, $\mathrm{Et}_{2} \mathrm{O}$, toluene, hexane and $\mathrm{CH}_{2} \mathrm{Cl}_{2}$ were dried by passage over activated alumina under nitrogen atmosphere $\left(\mathrm{H}_{2} \mathrm{O}\right.$ content $<7 \mathrm{ppm}$, Karl-Fischer titration). All chemicals were purchased and used as received unless stated otherwise. Chromatographic purification was performed as flash chromatography using Macherey-Nagel silica 40-63, $60 \AA$ A, using the solvents indicated as eluent with 0.1-0.5 bar pressure. TLC was performed on Merck silica gel 60 F254 TLC plastic or aluminium plates and visualized with UV light, permanganate CAN or p-anisaldehyde stains. Melting points were measured on a calibrated Büchi B-540 melting point apparatus using open glass capillaries. ${ }^{1} \mathrm{H}-\mathrm{NMR}$ spectra were recorded at room temperature on a Brucker DPX-400 $400 \mathrm{MHz}$ spectrometer in chloroform-d, all signals are reported in ppm with the internal chloroform signal at $7.26 \mathrm{ppm}$ or DMSO signal at $2.50 \mathrm{ppm}$ as standard. The data is being reported as $(\mathrm{s}=$ singlet, $\mathrm{d}=$ doublet, $\mathrm{t}=$ triplet, $\mathrm{q}=$ quadruplet, $\mathrm{qi}=$ quintet, $\mathrm{m}=$ multiplet or unresolved, br = broad signal, integration, coupling constant(s) in $\mathrm{Hz}$, interpretation). ${ }^{13} \mathrm{C}-\mathrm{NMR}$ spectra were recorded with 1H-decoupling on a Brucker DPX-400 $100 \mathrm{MHz}$ spectrometer in chloroform-d, all signals are reported in ppm with the internal chloroform signal at $77.0 \mathrm{ppm}$ or DMSO signal at $39.5 \mathrm{ppm}$ as standard. Infrared spectra were recorded on a JASCO FT-IR B4100 spectrophotometer with an ATR PRO410-S and a ZnSe prism and are reported as $\mathrm{cm}-1$ ( $\mathrm{w}=$ weak, $\mathrm{m}=$ medium, $\mathrm{s}=$ strong). High resolution mass spectrometric measurements were performed by the mass spectrometry service of ISIC at the EPFL on a MICROMASS (ESI) Q-TOF Ultima API. Iron-Alumina complex was synthesized following the procedure of Tietze. ${ }^{1}$ Diastereoisomeric mixtures have been assigned by $2 \mathrm{D}$ NMR experiments including COSY/ROESY/HSQC/HMBC. Commercialy available N-Vinyl Phthalimide [3485-84-5], N-Vinyl-2-pyrrolidone [88-12-0], Diethyl Ethylidenemalonate [1462-12-0], Benzaldehyde [100-52-7], 4-Chlorobenzaldehyde [104-88-1], 4-Methoxybenzaldehyde [123-11-5], 2Methoxybenzaldehyde [135-02-4], and trans-Cynnamaldehyde [14371-10-9] were used.

\footnotetext{
${ }^{1}$ Organic Syntheses, Vol. 71, p. 167 (1993); Coll. Vol. 9, p.310 (1998).
} 


\section{Synthesis of starting materials}

\subsection{Synthesis of Enamides and Alkylidene Malonates}

(E)-2-(Prop-1-en-1-yl)isoindoline-1,3-dione (12)

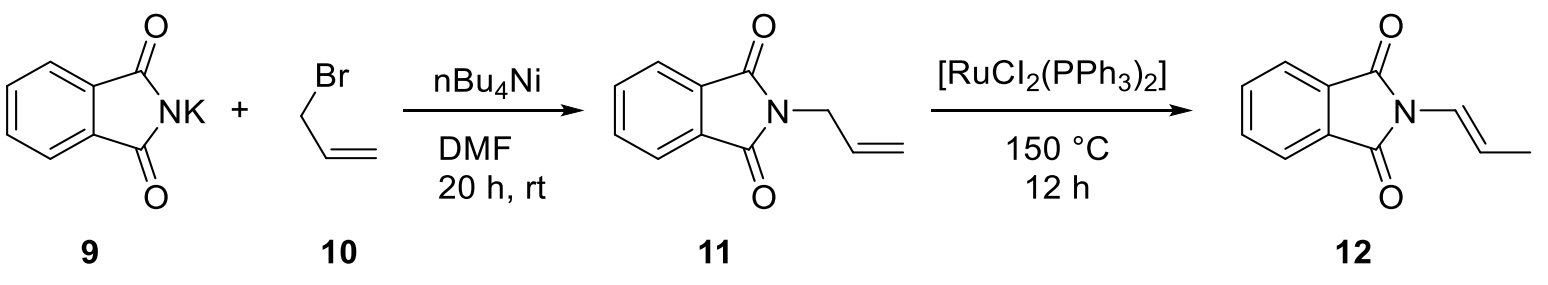

Following a modified procedure, ${ }^{2}$ allyl bromide (10) $(2.6 \mathrm{~mL}, 30 \mathrm{mmol}, 1.1 \mathrm{eq})$ was added dropwise at room temperature to a suspension of potassium phthalimide (9) $(5.0 \mathrm{~g}, 27 \mathrm{mmol}, 1 \mathrm{eq})$ and $\mathrm{Bu}_{4} \mathrm{NI}(0.50 \mathrm{~g}$, $1.4 \mathrm{mmol}, 0.05 \mathrm{eq})$ in DMF $(10 \mathrm{~mL})$. The mixture was stirred for $20 \mathrm{~h}$ at room temperature, and then $\mathrm{H}_{2} \mathrm{O}$ $(20 \mathrm{~mL})$ was added. The precipitate was isolated by filtration, dried, and recrystallized from isopropanol to give 2-allylisoindoline-1,3-dione (11) (3.4 g, $18 \mathrm{mmol}, 68 \%$ yield).

2-Allylisoindoline-1,3-dione (11) $(2.0 \mathrm{~g}, 11 \mathrm{mmol}, 1 \mathrm{eq})$ was added in a sealed tube under nitrogen atmosphere to $\left[\mathrm{RuCI}_{2}\left(\mathrm{PPh}_{3}\right)_{2}\right](0.10 \mathrm{~g}, 0.11 \mathrm{mmol}, 0.01 \mathrm{eq})$. The solids were heated at $150{ }^{\circ} \mathrm{C}$ during $12 \mathrm{~h}$ and the reaction was cooled down to room temperature. The black mixture was dissolved in toluene and filtered on a celite pad. The solvents were evaporated and the brown orange solid was recrystallized in ethanol (20 mL). (E)-2-(prop-1-en-1-yl)isoindoline-1,3-dione (12) (1.15 g, $6.10 \mathrm{mmol}, 58 \%$ yield) was collected as a yellow solid from the first recrystallization.

${ }^{1} \mathbf{H}$ NMR $\left(400 \mathrm{MHz}, \mathrm{CDCl}_{3}\right) \delta 7.86(\mathrm{dd}, 2 \mathrm{H}, J=5.2,3.1 \mathrm{~Hz}, \mathrm{Phth}), 7.72(\mathrm{dd}, 2 \mathrm{H}, J=5.2,3.0 \mathrm{~Hz}, \mathrm{Phth})$, 6.64-6.54 (m, $2 \mathrm{H}, \mathrm{CH}=\mathrm{CH}), 1.85\left(\mathrm{~d}, 3 \mathrm{H}, J=5.1 \mathrm{~Hz}, \mathrm{CH}_{3}\right)$.

${ }^{1} \mathrm{H}$ NMR data match literature report. ${ }^{2}$

\section{(E)-2-Styrylisoindoline-1,3-dione (14)}<smiles>C=CN1C(=O)c2ccccc2C1=O</smiles>

13

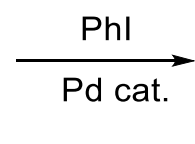

Following a reported procedure, ${ }^{3}$ a mixture of phenyl iodide $(816 \mathrm{mg}, 4.00 \mathrm{mmol}, 1 \mathrm{eq}), \mathrm{N}$ vinylphthalimide (13) $(693 \mathrm{mg}, 4.00 \mathrm{mmol}, 1 \mathrm{eq}), \mathrm{Cy}_{2} \mathrm{NMe}(1.17 \mathrm{~g}, 6.00 \mathrm{mmol}, 1.5 \mathrm{eq}), \mathrm{TBAB}(1.29 \mathrm{~g}$, $4.00 \mathrm{mmol}, 1 \mathrm{eq})$ and palladium acetate $(1.00 \mathrm{mg}, 4.00 \mu \mathrm{mol}, 0.001 \mathrm{eq})$ in DMF $(8 \mathrm{~mL})$ was heated at 120 ${ }^{\circ} \mathrm{C}$ (oil bath temperature) in a pressure tube after 5 cycles of vacuum/ $\mathrm{N}_{2}$. After $1.5 \mathrm{~h}$, the yellow solution was poured into toluene $(40 \mathrm{~mL})$ and rinsed with toluene $(10 \mathrm{~mL})$. This solution was filtered through a pad of celite and concentrated under vacuum. To the yellow oil was added ethanol $(20 \mathrm{~mL})$. The precipitated solid was recovered by filtration and rinsed with ethanol. (E)-2-styrylisoindoline-1,3-dione (14) (700 mg, $2.77 \mathrm{mmol}, 69 \%$ yield) was obtained as a yellow powder.

${ }^{1}$ H NMR (400 MHz, $\mathrm{CDCl}_{3}$ ) $\delta 7.91$ (dd, $2 \mathrm{H}, J=5.5,3.1 \mathrm{~Hz}$, Phth), 7.77 (dd, $2 \mathrm{H}, J=5.4,3.1 \mathrm{~Hz}$, Phth), $7.66(\mathrm{~d}, 1 \mathrm{H}, J=15.2 \mathrm{~Hz}, \mathrm{CH}=\mathrm{CH}), 7.51-7.45(\mathrm{~m}, 2 \mathrm{H}, \mathrm{ArH}), 7.40-7.32(\mathrm{~m}, 3 \mathrm{H}, \mathrm{ArH}), 7.30-7.24(\mathrm{~m}, 1 \mathrm{H}$, $\mathrm{CH}=\mathrm{CH})$.

Data match literature report. ${ }^{3}$

${ }^{2}$ Stojanovic, A.; Renaud, P.; Schenk, K. Helv. Chim. Acta, 2004, 81, 268.

${ }^{3}$ Alacid, E.; Nájera, C. Adv. Synth. Catal. 2008, 350, 1316. 


\section{1-Vinylthymine (16).}<smiles>Cc1c[nH]c(=O)[nH]c1=O</smiles>

15

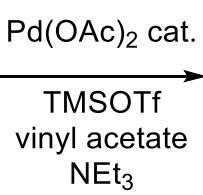

$\mathrm{NEt}_{3}$<smiles>C=Cn1cc(C)c(=O)[nH]c1=O</smiles>

16

Following the described procedure of Racine et $\mathrm{al}^{4}$, thymine (15) $(0.50 \mathrm{~g}, 3.9 \mathrm{mmol}, 1.0 \mathrm{eq})$, diacetoxypalladium $(0.036 \mathrm{~g}, 0.16 \mathrm{mmol}, 0.04 \mathrm{eq})$ were suspended in DMF $(10 \mathrm{~mL})$ in a flame-dried sealed microwave vial under nitrogen atmosphere. Vinyl acetate $(0.82 \mathrm{~g}, 9.5 \mathrm{mmol}$, and $2.4 \mathrm{eq})$, triethylamine $(1.3 \mathrm{~mL}, 9.5 \mathrm{mmol}, 2.4 \mathrm{eq})$ and TMSOTf $(1.7 \mathrm{~mL}, 9.5 \mathrm{mmol}$, and $2.4 \mathrm{eq})$ were added to the reaction mixture and stirred at $70{ }^{\circ} \mathrm{C}$ for 24 hours. The reaction mixture was cooled down to room temperature and the solvent was removed under reduced pressure. The crude mixture was purified by column chromatography, eluting with a mixture of ethyl acetate/ pentane (7:3). The pure 1-vinylthymine (16) ( $0.44 \mathrm{~g}, 2.8 \mathrm{mmol}, 73 \%$ yield) was obtained as white solid.

$\mathbf{R}_{\boldsymbol{f}} 0.32$ (3:7 Pentane/Ethyl acetate).

${ }^{1}$ H NMR (400 MHz, Chloroform- $d$ ) $\delta 8.52(\mathrm{~s}, 1 \mathrm{H}, \mathrm{NH}), 7.34(\mathrm{~d}, J=1.3 \mathrm{~Hz}, 1 \mathrm{H}$, thymine C=C-H), 7.20 $(\mathrm{dd}, J=16.0,9.1 \mathrm{~Hz}, 1 \mathrm{H} \text {, vinyl-CH), } 5.07 \text { (dd, } J=16.0,2.2 \mathrm{~Hz}, 1 \mathrm{H} \text {, vinyl-CH})_{2}, 4.92$ (dd, $J=9.1,2.2$ $\mathrm{Hz}, 1 \mathrm{H}$, vinyl- $\left.\mathrm{CH}_{2}\right), 1.99\left(\mathrm{~d}, J=1.3 \mathrm{~Hz}, 3 \mathrm{H}\right.$, thymine- $\left.\mathrm{CH}_{3}\right)$.

${ }^{13}$ C NMR (101 MHz, Chloroform- $d$ ) $\delta$ 163.4, 149.2, 134.6, 129.7, 112.2, 100.7, 12.7.

NMR values correspond to the literature. ${ }^{4}$

\section{1-Vinylfluorouracil (18)}<smiles>O=c1[nH]cc(F)c(=O)[nH]1</smiles>

17

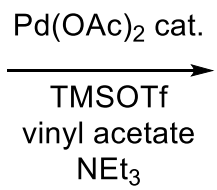

$\mathrm{NEt}_{3}$<smiles>C=Cn1cc(F)c(=O)[nH]c1=O</smiles>

18

Following the described procedure of Racine et al., ${ }^{4}$ fluorouracil (17) $(0.52 \mathrm{~g}, 3.9 \mathrm{mmol}, 1.0 \mathrm{eq})$ and diacetoxypalladium $(0.036 \mathrm{~g}, 0.16 \mathrm{mmol}, 0.04 \mathrm{eq})$ were suspended in DMF $(10 \mathrm{~mL})$ in a flame-dried sealed microwave vial under nitrogen atmosphere. Vinyl acetate $(0.82 \mathrm{~g}, 9.5 \mathrm{mmol}, 2.4 \mathrm{eq})$, triethylamine $(1.3 \mathrm{~mL}, 9.5 \mathrm{mmol}, 2.4 \mathrm{eq})$ and TMSOTf $(1.7 \mathrm{~mL}, 9.5 \mathrm{mmol}, 2.4 \mathrm{eq})$ were added to the reaction mixture and stirred at $70^{\circ} \mathrm{C}$ for 24 hours. The reaction mixture was cooled down to room temperature and the solvent was removed under reduced pressure. The crude mixture was purified by column chromatography, eluting with a mixture of ethyl acetate/ pentane (5:5). The pure 1-vinylfluorouracil (18) $(0.31 \mathrm{~g}, 2.0 \mathrm{mmol}$, $50 \%$ yield) was obtained as white solid.

$\mathbf{R}_{f} 0.35$ (6:4 Pentane/Ethyl acetate).

${ }^{1}$ H NMR (400 MHz, DMSO-d6) $\delta 12.01$ (s, 1H, N H), 8.43 (d, $J=7.1 \mathrm{~Hz}, 1 \mathrm{H}$, F-uracil C=C-H), 7.07 $\left(\mathrm{ddd}, J=16.0,9.2,1.9 \mathrm{~Hz}, 1 \mathrm{H}\right.$, vinyl-CH), $5.36\left(\mathrm{dd}, J=15.9,2.0 \mathrm{~Hz}, 1 \mathrm{H}\right.$, vinyl- $\left.\mathrm{CH}_{2}\right), 4.88(\mathrm{dd}, J=9.2$, $2.0 \mathrm{~Hz}, 1 \mathrm{H}$, vinyl- $\mathrm{CH}_{2}$ ).

\footnotetext{
${ }^{4}$ Racine, S.; de Nanteuil, F.; Serrano, E.; Waser, J. Angew. Chem. Int. Ed. 2014, 53, 8484-8487.
} 
${ }^{1} \mathrm{H}$ NMR values are in accordance with the spectra performed in chloroform in the literature. ${ }^{4}$

Dimethyl 2-methylenemalonate (20)

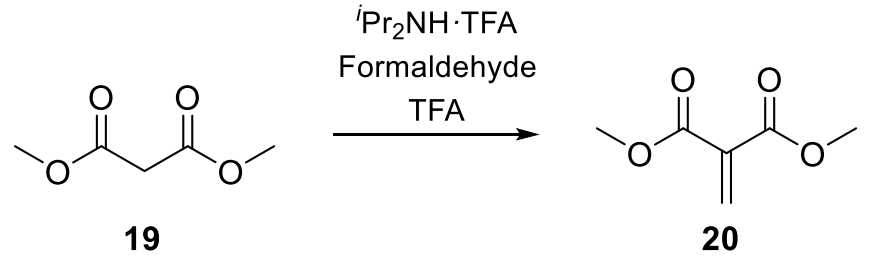

Following the described procedure of De Nanteuil et al., ${ }^{5}$ paraformaldehyde $(2.7 \mathrm{~g}, 90 \mathrm{mmol}, 2.0 \mathrm{eq})$ and diisopropylamine 2,2,2-trifluoroacetate $(9.7 \mathrm{~g}, 45 \mathrm{mmol}, 1.0 \mathrm{eq})$ were added in a flame dried $250 \mathrm{~mL}$ round flask with a condenser. Then, tetrahydrofuran $(65 \mathrm{~mL})$, dimethyl malonate $(19)(5.1 \mathrm{~mL}, 45 \mathrm{mmol}$, $1.0 \mathrm{eq})$ and trifluoroacetic acid $(0.35 \mathrm{~mL}, 4.5 \mathrm{mmol}, 0.1 \mathrm{eq})$ were added into the flask under nitrogen atmosphere and the suspension was stirred to reflux for 2 hours. Paraformaldehyde $(2.7 \mathrm{~g}, 90 \mathrm{mmol}, 2.0$ eq) was added to the reaction mixture and the reflux was restarted for 12 hours. The reaction mixture was cooled to room temperature and THF was removed under reduced pressure. The crude product was dissolved in diethyl ether $(25 \mathrm{~mL})$ and filtrated trough cotton. The organic layer was washed two times with $1 \mathrm{M} \mathrm{HCl}(25 \mathrm{~mL})$, the aqueous layers were combined and washed three times with diethyl ether (50 $\mathrm{mL})$. The combined organic layers were dry over magnesium sulfate, filtrated and concentrated under reduced pressure. The crude mixture containing 20 was obtained as colorless oil (2.5 g).

${ }^{1}$ H NMR (400 MHz, Chloroform- $d$ ) $\delta 6.58\left(\mathrm{~s}, 2 \mathrm{H}, \mathrm{CH}_{2}\right.$ ), 3.83 (s, $6 \mathrm{H}$, ester $\mathrm{CH}_{3}$ ).

${ }^{1} \mathrm{H}$ NMR values correspond to the literature. ${ }^{5}$

\subsection{Synthesis of Cyclobutanes}

\section{Dimethyl 2-(1,3-dioxoisoindolin-2-yl)cyclobutane-1,1-dicarboxylate (4a)}

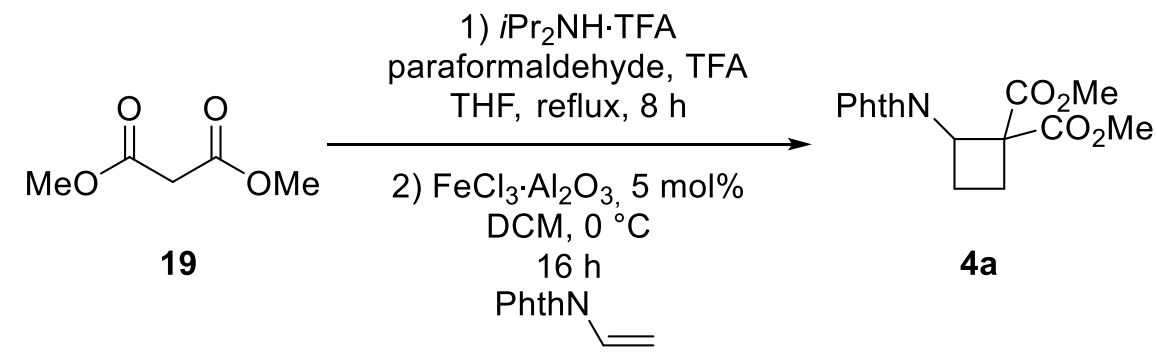

13

Following a reported procedure, ${ }^{5}$ dimethyl malonate (19) $(1.32 \mathrm{~mL}, 11.6 \mathrm{mmol}, 2 \mathrm{eq})$, diisopropylamine 2,2,2-trifluoroacetate $(2.49 \mathrm{~g}, 11.6 \mathrm{mmol}, 2 \mathrm{eq})$, paraformaldehyde $(0.695 \mathrm{mg}, 23.1 \mathrm{mmol}, 4 \mathrm{eq})$ and trifluoroacetic acid $(89.0 \mu \mathrm{L}, 1.16 \mathrm{mmol}, 0.2 \mathrm{eq})$ were added to tetrahydrofuran $(20 \mathrm{~mL})$. A condenser was added and the suspension was stirred at reflux for $2 \mathrm{~h}$. Paraformaldehyde $(0.695 \mathrm{mg}, 23.1 \mathrm{mmol}, 4 \mathrm{eq})$ was added and the reflux was continued for $6 \mathrm{~h}$. The reaction was cooled to room temperature and the tetrahydrofuran was removed under reduced pressure $\left(300\right.$ to $50 \mathrm{mbar}$ at $\left.45^{\circ} \mathrm{C}\right)$. The crude was dissolved in diethyl ether $(25 \mathrm{~mL})$ and filtered through cotton in a separatory funnel. The organic layer was washed twice with $1 \mathrm{M} \mathrm{HCl}(25 \mathrm{~mL})$. The aqueous layers were combined and extracted with diethyl ether (25 $\mathrm{mL}$ ). The organic layers were combined, dried over anhydrous $\mathrm{Na}_{2} \mathrm{SO}_{4}$, filtered and concentrated under reduced pressure to give dimethyl 2-methylenemalonate crude (20) as colorless oil.

${ }^{5}$ De Nanteuil, F.; Waser, J. Angew. Chem. Int. Ed. 2013, 52, 9009-9013. 
The iron trichloride supported on alumina $(1.00 \mathrm{mmol} / \mathrm{g}, 289 \mathrm{mg}, 0.289 \mathrm{mmol}, 0.05 \mathrm{eq})$ was weighted in an oven-dry flask in a glovebox. The flask was closed with a silicon septum, taken out of the glovebox and put under positive pressure of nitrogen and dichloromethane $(5 \mathrm{~mL})$ was added. 2-vinylisoindoline-1,3dione (13) (1.00 g, $5.77 \mathrm{mmol}, 1 \mathrm{eq})$ was dissolved in dichloromethane $(5 \mathrm{~mL})$ and added to the yellow suspension. Finally, the crude dimethyl 2-methylenemalonate (20) was dissolved in dichloromethane (5 $\mathrm{mL}$ ) and added to the reaction in one portion. The reaction was stirred at room temperature for $16 \mathrm{~h}$ and then filtered over a basic alumina plug, eluting with ethyl acetate. The solvents were evaporated and the brown solid was purified by column chromatography on Biotage (SNAP cartridge KP-SIL 50 g, 95:5 to 4:6 Hexane/Ethyl acetate) affording cyclobutane $\mathbf{4 a}(1.55 \mathrm{~g}, 4.89 \mathrm{mmol}, 85 \%$ yield) as a colorless solid.

$\mathbf{R}_{\mathbf{f}} 0.45$ (1:1 Hexane/Ethyl acetate).

${ }^{1} \mathbf{H}$ NMR (400 MHz, $\left.\mathrm{CDCl}_{3}\right) \delta 7.93$ (m, $\left.2 \mathrm{H}, \mathrm{Phth}\right), 7.80$ (m, $\left.2 \mathrm{H}, \mathrm{Phth}\right), 5.17$ (t, $\left.1 \mathrm{H}, J=10.9 \mathrm{~Hz}, \mathrm{~N}-\mathrm{C}-\mathrm{H}\right)$, $3.16\left(\mathrm{~s}, 3 \mathrm{H}, \mathrm{CO}_{2} \mathrm{CH}_{3}\right), 2.98\left(\mathrm{~s}, 3 \mathrm{H}, \mathrm{CO}_{2} \mathrm{CH}_{3}\right), 2.58\left(\mathrm{~m}, 1 \mathrm{H}, \mathrm{CH}_{2}\right), 2.25\left(\mathrm{~m}, 1 \mathrm{H}, \mathrm{CH}_{2}\right), 1.48(\mathrm{~m}, 1 \mathrm{H}$, $\left.\mathrm{CH}_{2}\right), 1.33\left(\mathrm{dt}, 1 \mathrm{H}, J=13.6,10.4 \mathrm{~Hz}, \mathrm{CH}_{2}\right)$.

${ }^{13} \mathbf{C}$ NMR $\left(101 \mathrm{MHz}, \mathrm{CDCl}_{3}\right) \delta 170.6,168.7,168.3,134.3,131.9,123.5,59.0,53.2,53.0,47.9,24.7,21.9$.

Data match literature report. ${ }^{5}$

Dimethyl 2-(1,3-dioxoisoindolin-2-yl)-3-methylcyclobutane-1,1-dicarboxylate (4b)

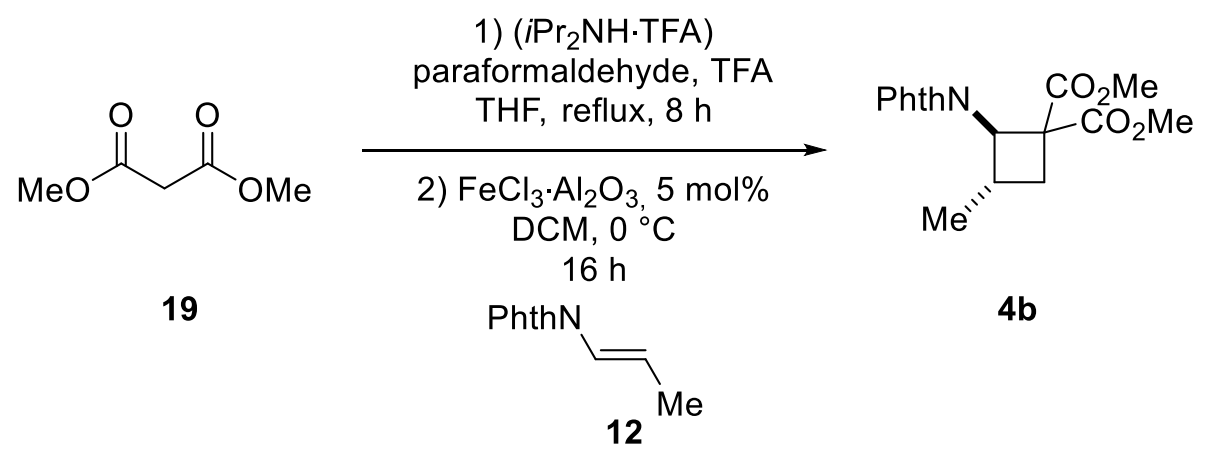

Following a reported procedure, ${ }^{5}$ dimethyl malonate $(\mathbf{1 9})(1.22 \mathrm{~mL}, 10.7 \mathrm{mmol}, 2 \mathrm{eq})$, diisopropylamine 2,2,2-trifluoroacetate $(2.30 \mathrm{~g}, 10.7 \mathrm{mmol}, 2 \mathrm{eq})$, paraformaldehyde $(0.640 \mathrm{mg}, 21.4 \mathrm{mmol}, 4 \mathrm{eq})$ and trifluoroacetic acid $(82.0 \mu 1,1.07 \mathrm{mmol}, 0.2 \mathrm{eq})$ were added to tetrahydrofuran $(20 \mathrm{~mL})$. A condenser was added and the suspension was stirred at reflux for $2 \mathrm{~h}$. Paraformaldehyde $(0.640 \mathrm{mg}, 21.4 \mathrm{mmol})$ was added and the reflux was continued for $6 \mathrm{~h}$. The reaction was cooled to room temperature and the tetrahydrofuran was removed under reduced pressure. The crude was dissolved in diethyl ether $(25 \mathrm{~mL})$ and filtered through cotton in a separatory funnel. The organic layer was washed twice with $1 \mathrm{M} \mathrm{HCl} \mathrm{(25}$ $\mathrm{mL})$. The aqueous layers were combined and extracted with diethyl ether $(25 \mathrm{~mL})$. The organic layers were combined, dried over anhydrous $\mathrm{Na}_{2} \mathrm{SO}_{4}$, filtered and concentrated under reduced pressure to give dimethyl 2-methylenemalonate crude as colorless oil.

The iron trichloride supported on alumina $(1.00 \mathrm{mmol} / \mathrm{g}, 267 \mathrm{mg}, 0.267 \mathrm{mmol}, 0.05 \mathrm{eq})$ was weighted in an oven-dry flask in a glovebox. The flask was closed with a silicon septum, taken out of the glovebox and put under a positive pressure of nitrogen. Dichloromethane $(5 \mathrm{~mL})$ was added. The reaction was cooled to $0{ }^{\circ} \mathrm{C}$ and $(E)$-2-(prop-1-en-1-yl)isoindoline-1,3-dione (12) $(1.00 \mathrm{~g}, 5.34 \mathrm{mmol}, 1 \mathrm{eq})$ was dissolved in dichloromethane $(5 \mathrm{~mL})$ and added to the yellow suspension dropwise. Finally, the crude dimethyl 2methylenemalonate was dissolved in dichloromethane $(5 \mathrm{~mL})$ and added to the reaction mixture dropwise. The reaction mixture was stirred at room temperature for $4 \mathrm{~h}$ and then filtered over a basic alumina plug, eluting with ethyl acetate. The solvents were evaporated and the brown solid was purified by column 
chromatography on Biotage (SNAP cartridge KP-SIL 50 g, 95:5 to 4:6 Hexane/Ethyl acetate) affording cyclobutane $4 \mathbf{b}(1.53 \mathrm{~g}, 4.62 \mathrm{mmol}, 86 \%$ yield $)$ as a colorless solid.

$\mathbf{R}_{\mathbf{f}} 0.26$ (7:3 Hexane/Ethyl acetate).

Mp 112.1-114. ${ }^{\circ} \mathrm{C}$.

${ }^{1} \mathbf{H}$ NMR $\left(400 \mathrm{MHz}, \mathrm{CDCl}_{3}\right) \delta 7.83$ (m, $\left.2 \mathrm{H}, \mathrm{Phth}\right), 7.72$ (m, $\left.2 \mathrm{H}, \mathrm{Phth}\right), 4.96$ (d, $1 \mathrm{H}, J=10.3 \mathrm{~Hz}, \mathrm{~N}-\mathrm{C}-$ $\mathrm{H}), 3.71\left(\mathrm{~m}, 4 \mathrm{H}, \mathrm{CO}_{2} \mathrm{CH}_{3}\right.$ and $\left.\mathrm{C}-\mathrm{H}-\mathrm{CH}_{3}\right), 3.60\left(\mathrm{~s}, 3 \mathrm{H}, \mathrm{CO}_{2} \mathrm{CH}_{3}\right), 3.00\left(\mathrm{dd}, 1 \mathrm{H}, J=10.9,9.3 \mathrm{~Hz}, \mathrm{CH}_{2}\right)$, $1.74\left(\mathrm{dd}, 1 \mathrm{H}, J=11.5,9.5 \mathrm{~Hz}, \mathrm{CH}_{2}\right), 1.16\left(\mathrm{~d}, 3 \mathrm{H}, J=6.6 \mathrm{~Hz}, \mathrm{CH}_{3}\right)$.

${ }^{13}$ C NMR $\left(101 \mathrm{MHz}, \mathrm{CDCl}_{3}\right) \delta 170.4,168.6,168.3,134.2,131.8,123.4,56.3,54.9,53.0,52.9,32.1,29.9$, 19.3 .

Data match literature report. ${ }^{5}$

Dimethyl 2-(1,3-dioxoisoindolin-2-yl)-3-phenylcyclobutane-1,1-dicarboxylate (4c)

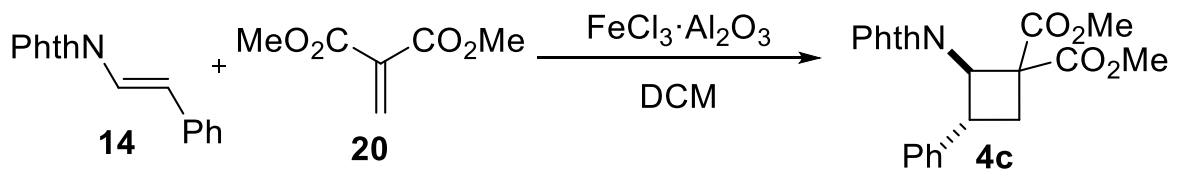

Following a slightly modified procedure, ${ }^{5}$ in the glovebox, iron trichloride supported on alumina (1.00 $\mathrm{mmol} / \mathrm{g}, 282 \mathrm{mg}, 0.282 \mathrm{mmol}, 0.05 \mathrm{eq})$ was added to a microwave vial. The vial was sealed with a Teflon septum and taken out of the glovebox. Dry dichloromethane $(5 \mathrm{~mL})$ was added and $(E)-2-$ styrylisoindoline-1,3-dione (14) (1.41 g, $5.64 \mathrm{mmol}, 1 \mathrm{eq})$ was dissolved in dry dichloromethane $(5 \mathrm{~mL})$ and added to the iron trichloride. Dimethyl 2-methylenemalonate (20) (1.63 g, $11.3 \mathrm{mmol}, 2 \mathrm{eq})$ was dissolved in dry dichloromethane $(5 \mathrm{~mL})$ and added dropwise over $2 \mathrm{~h}$. When the addition was finished, the reaction was stirred for $4 \mathrm{~h}$ at room temperature. Then, dimethyl 2-methylenemalonate (406 mg, 2.82 mmol, 0.5 eq) was dissolved in dry dichloromethane $(2 \mathrm{~mL})$ and added in one portion. The reaction was stirred at reflux for $2 \mathrm{~h}$, and then filtered over a pad of alumina, eluted with ethyl acetate and concentrated under vacuum. The crude product was purified by column chromatography $\left(\mathrm{SiO}_{2}, 9: 1\right.$ to 7:3 Pentane/Ethyl Acetate) affording cyclobutane $\mathbf{4 c}(1.74 \mathrm{~g}, 4.42 \mathrm{mmol}, 78 \%$ yield) as a colorless oil.

$\mathbf{R}_{\mathbf{f}} 0.28$ (7:3 Hexane/Ethyl acetate).

${ }^{1}$ H NMR $\left(400 \mathrm{MHz}, \mathrm{CDCl}_{3}\right) \delta 7.63(\mathrm{dd}, 2 \mathrm{H}, J=3.7,2.1 \mathrm{~Hz}$, Phth), 7.55 (dd, $2 \mathrm{H}, J=3.8,2.1 \mathrm{~Hz}$, Phth), 7.29-7.24 (m, $4 \mathrm{H}, \mathrm{Ph}), 7.23-7.19(\mathrm{~m}, 1 \mathrm{H}, \mathrm{Ph}), 5.52(\mathrm{dd}, 1 \mathrm{H}, J=7.4,0.4 \mathrm{~Hz}, \mathrm{~N}-\mathrm{C}-\mathrm{H}), 4.86-4.96(\mathrm{~m}, 1 \mathrm{H}$, $H$-C-Ph), 3.76 (s, $3 \mathrm{H}, \mathrm{CO}_{2} \mathrm{CH}_{3}$ ), 3.65 (s, $3 \mathrm{H}, \mathrm{CO}_{2} \mathrm{CH}_{3}$ ), 3.25 (ddd, $1 \mathrm{H}, J=7.7,6.5,0.4 \mathrm{~Hz}, \mathrm{CH}_{2}$ ), 2.21 (dd, $1 \mathrm{H}, J=7.8,6.9 \mathrm{~Hz}, \mathrm{CH}_{2}$ ).

${ }^{13}$ C NMR $\left(101 \mathrm{MHz}, \mathrm{CDCl}_{3}\right) \delta 158.8,157.4,157.2,134.0,128.8,126.6,124.1,122.9,122.5,119.7,63.1$, 61.4, 60.7, 60.7, 48.4, 42.9.

Data match literature report. ${ }^{5}$

\section{Diethyl 2-(1,3-dioxoisoindolin-2-yl)-4-methylcyclobutane-1,1-dicarboxylate (4d)}

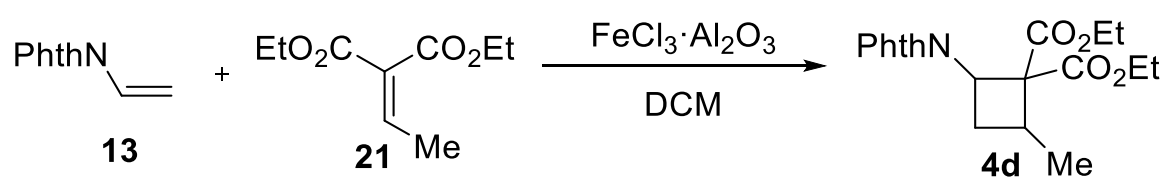

Following a slightly modified procedure, ${ }^{5}$ in the glovebox, iron trichloride supported on alumina (1.00 $\mathrm{mmol} / \mathrm{g}, 289 \mathrm{mg}, 0.289 \mathrm{mmol}, 0.1 \mathrm{eq})$ was added to a microwave vial. The vial was sealed with a Teflon septum and taken out of the glovebox. Dry dichloromethane $(3 \mathrm{~mL})$ was added. Diethyl 2- 
ethylidenemalonate (21) (1.07 g, $5.77 \mathrm{mmol}, 2 \mathrm{eq})$ and 2-vinylisoindoline-1,3-dione (13) (500 mg, 2.89 mmol, $1 \mathrm{eq})$ were dissolved in dry dichloromethane $(4 \mathrm{~mL})$. The solution was then added dropwise to the iron trichloride. The reaction was stirred at $40{ }^{\circ} \mathrm{C}$ for $16 \mathrm{~h}$. Then the reaction was filtered over a pad of alumina, eluted with ethyl acetate and concentrated under vacuum. The crude product was purified by column chromatography $\left(\mathrm{SiO}_{2}, 8: 2\right.$ Pentane/Ethyl Acetate), affording cyclobutane 4d (806 mg, 2.24 mmol, $78 \%$ yield, $1.1: 1 \mathrm{dr}$ determined by integration of the peaks at 5.58 (maj) and 4.91 ( $\mathrm{min}$ ) in the crude ${ }^{1} \mathrm{H}$ NMR, as a pale yellow oil.

$\mathbf{R}_{\mathbf{f}} 0.25$ (7:3 Hexane/Ethyl acetate).

${ }^{1} \mathbf{H}$ NMR $\left(400 \mathrm{MHz}, \mathrm{CDCl}_{3}\right)$ on a 1.1(maj):1(min) mixture $\delta$ 7.84-7.79 (m, $3.8 \mathrm{H}$, Phth maj + min), 7.727.67 (m, 3.8 H, Phth maj + min), 5.58 (ddd, $J=10.0,8.3,1.2 \mathrm{~Hz}, 1 \mathrm{H}, \mathrm{N}-\mathrm{C}-\mathrm{H}$ min), 4.91 (dd, $J=10.9,8.7$ $\mathrm{Hz}, 0.9 \mathrm{H}, \mathrm{N}-\mathrm{C}-\mathrm{H}$ min), 4.27-4.18 (m, 3.8 H, $\mathrm{CO}_{2} \mathrm{CH}_{2}$ maj + min), 4.17- $3.91\left(\mathrm{~m}, 3.8 \mathrm{H}, \mathrm{CO}_{2} \mathrm{CH}_{2}\right.$ maj + min), 3.65-3.54 (m, $1 \mathrm{H}, \mathrm{CH}$ cyclobutane maj), 3.27-3.12 (m, $1.9 \mathrm{H}, \mathrm{CH}_{2}$ cyclobutane maj + min), 2.78 (ddq, $J=10.7,8.1,7.0 \mathrm{~Hz}, 0.9 \mathrm{H}, \mathrm{CH}$ cyclobutane $\min$ ), 2.46 (dt, $J=10.8,8.5 \mathrm{~Hz}, 0.9 \mathrm{H}, \mathrm{CH}_{2}$ cyclobutane min), 1.98 (ddd, $J=11.7,10.0,5.4 \mathrm{~Hz}, 1 \mathrm{H}, \mathrm{CH}_{2}$ cyclobutane maj), $1.32\left(\mathrm{~d}, J=7.0 \mathrm{~Hz}, 2.7 \mathrm{H}, \mathrm{CH}_{3}\right.$ min), 1.28-1.21 (m, $\left.5.7 \mathrm{H}, \mathrm{CO}_{2} \mathrm{CH}_{2} \mathrm{CH}_{3} m a j+m i n\right), 1.14\left(\mathrm{~d}, J=7.3 \mathrm{~Hz}, 3 \mathrm{H}, \mathrm{CH}_{3}\right.$ maj), 1.05 (t, $J=7.1 \mathrm{~Hz}, 2.7$ $\mathrm{H}, \mathrm{CO}_{2} \mathrm{CH}_{2} \mathrm{CH}_{3}$ min), 0.90 (t, $J=7.1 \mathrm{~Hz}, 3 \mathrm{H}, \mathrm{CO}_{2} \mathrm{CH}_{2} \mathrm{CH}_{3}$ maj).

${ }^{13} \mathrm{C}$ NMR $\left(101 \mathrm{MHz}, \mathrm{CDCl}_{3}\right) \delta 170.2,168.5,168.4,168.3,168.1,167.5,134.1,134.0,132.0,131.8$, $123.3,123.2,62.4,62.0,61.7,{ }^{6} 61.6,60.9,47.5,45.1,33.8,31.1,29.6,28.7,16.5,16.2,14.2,14.0,13.9$, 13.6.

Data match literature report. ${ }^{5}$

\section{Dimethyl 2-(5-methyl-2,4-dioxo-3,4-dihydropyrimidin-1(2H)-yl)cyclobutane-1,1-dicarboxylate (22)}

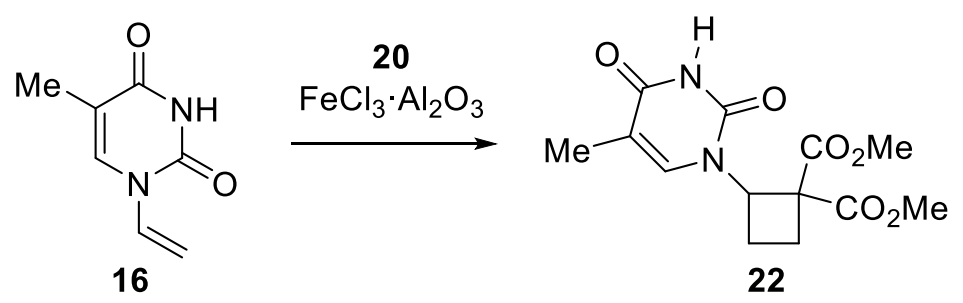

Following an adapted described procedure of De Nanteuil et al., ${ }^{5}$ 5-methyl-1-vinylpyrimidine-2,4(1H,3H)dione (16) (0.43 g, $2.9 \mathrm{mmol}, 1.0 \mathrm{eq})$ and iron trichloride supported on alumina $(1.0 \mathrm{mmol} / \mathrm{g}, 0.57 \mathrm{~g}, 0.57$ mmol, 0.2 eq) were added in a flame dried microwave vial under nitrogen atmosphere. Then, dry dichloromethane $(7 \mathrm{~mL})$ and a solution of crude freshly prepared dimethyl 2-methylenemalonate (20) $(0.82 \mathrm{~g}, 5.7 \mathrm{mmol}, 2.0 \mathrm{eq})$ in dichloromethane $(3 \mathrm{~mL})$ were added into the vial and the reaction mixture was stirred at room temperature for 14 hours. The reaction mixture was filtrated over a pad of alumina eluting with ethyl acetate $(25 \mathrm{~mL})$ and concentrated under reduced pressure. The crude mixture was purified via column chromatography, eluting with a mixture of ethyl acetate/ pentane (6:4 to 9:1). The pure product 22 ( $0.36 \mathrm{~g}, 1.2 \mathrm{mmol}, 43 \%$ yield) was obtained as a white solid.

$\mathbf{R}_{f} 0.21$ ( $2: 8$ Pentane/Ethyl acetate).

Mp $180.4-182.1^{\circ} \mathrm{C}$.

${ }^{1}$ H NMR (400 MHz, Chloroform- $d$ ) $\delta 7.99(\mathrm{~s}, 1 \mathrm{H}, \mathrm{NH}), 7.05$ (d, $J=1.3 \mathrm{~Hz}, 1 \mathrm{H}$, thymine C=C-H), 5.33 (t, $J=9.2 \mathrm{~Hz}, 1 \mathrm{H}$, cyclobutane-CH), $3.77\left(\mathrm{~s}, 3 \mathrm{H}\right.$, ester $\left.\mathrm{CH}_{3}\right), 3.69\left(\mathrm{~s}, 3 \mathrm{H}\right.$, ester $\left.\mathrm{CH}_{3}\right), 2.79-2.67(\mathrm{~m}, 2$ $\mathrm{H}$, cyclobutane- $\left.\mathrm{CH}_{2}\right), 2.35$ (q, $J=8.5 \mathrm{~Hz}, 1 \mathrm{H}$, cyclobutane- $\left.\mathrm{CH}_{2}\right), 2.27-2.16\left(\mathrm{~m}, 1 \mathrm{H}\right.$, cyclobutane- $\left.\mathrm{CH}_{2}\right)$, $1.93\left(\mathrm{~d}, J=1.2 \mathrm{~Hz}, 3 \mathrm{H}\right.$, thymine- $\left.\mathrm{CH}_{3}\right)$.

${ }^{6}$ Two peaks under this signal as determined by HMBC. 
${ }^{13}$ C NMR (101 MHz, Chloroform- $\left.d\right) \delta$ 170.0, 168.8, 163.9, 151.0, 137.7, 110.3, 59.1, 55.0, 53.2, 53.1, $23.7,22.8,12.5$.

IR 3190 (w), 3034 (w), 2958 (w), 2361 (m), 1733 (s), 1690 (s), 1439 (w), 1278 (m), 1113 (w), 915 (w), $735(\mathrm{w})$.

HRMS (ESI) calcd for $\mathrm{C}_{13} \mathrm{H}_{16} \mathrm{~N}_{2} \mathrm{NaO}_{6}{ }^{+}[\mathrm{M}+\mathrm{Na}]^{+} 319.0901$, found 319.0904 .

Dimethyl 2-(3-benzoyl-5-methyl-2,4-dioxo-3,4-dihydropyrimidin-1(2H)-yl)cyclobutane-1,1dicarboxylate (4e).<smiles>COC(=O)C1(C(C)=O)CCC1n1cc(C)c(=O)[nH]c1=O</smiles>

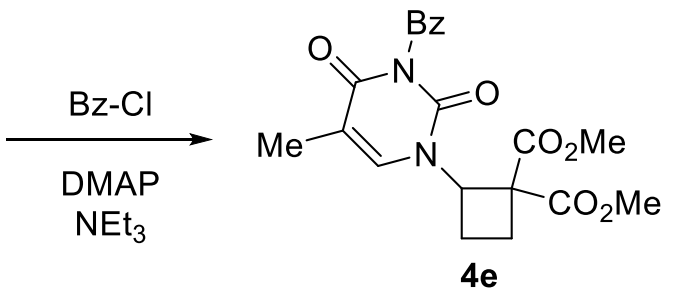

Following an adapted described procedure of Racine et al., ${ }^{4}$ in a flame dried microwave vial under nitrogen atmosphere, cyclobutane $22(0.32 \mathrm{~g}, 1.1 \mathrm{mmol}, 1.0 \mathrm{eq})$ and dimethyl-4-aminopyridine $(0.13 \mathrm{~g}$, $1.1 \mathrm{mmol}, 1.0 \mathrm{eq})$ were dissolved in dry dichloromethane $(10 \mathrm{~mL})$. Then, triethylamine $(0.60 \mathrm{~mL}, 4.3$ mmol, 4.0 eq) was added and the solution was cooled to $0{ }^{\circ} \mathrm{C}$. Benzoyl chloride $(0.38 \mathrm{~mL}, 3.2 \mathrm{mmol}, 3.0$ eq) was added dropwise and the reaction mixture was stirred at room temperature for 14 hours. A saturated solution of $\mathrm{NaHCO}_{3}(8 \mathrm{~mL})$ was added to the reaction mixture and the layers were separated. The aqueous layer was extracted with dichloromethane $(2 \times 20 \mathrm{~mL})$. The combined organic layers were washed with $1 \mathrm{M} \mathrm{HCl}(2 \times 40 \mathrm{~mL})$ and with water $(30 \mathrm{~mL})$, dried over $\mathrm{MgSO}_{4}$ and concentrated under reduced pressure. The crude product was purified by column chromatography, eluting with a mixture of pentane/ ethyl acetate (4:6 to 2:8). The pure product $4 \mathbf{e}(0.37 \mathrm{~g}, 0.93 \mathrm{mmol}, 86 \%$ yield) was obtained as colorless foam.

$\mathbf{R}_{f} 0.32$ (4:6 Pentane/Ethyl acetate).

Mp $153.8-155.4^{\circ} \mathrm{C}$.

${ }^{1}$ H NMR (400 MHz, Chloroform- $d$ ) $\delta 7.96$ - 7.90 (m, $2 \mathrm{H}$, Ar-H), 7.65 - 7.59 (m, $\left.1 \mathrm{H}, \mathrm{Ar}-\mathrm{H}\right), 7.50$ - 7.44 $(\mathrm{m}, 2 \mathrm{H}, \mathrm{Ar}-\mathrm{H}), 7.16(\mathrm{~d}, J=1.2 \mathrm{~Hz}, 1 \mathrm{H}$, thymine $\mathrm{C}=\mathrm{C}-\mathrm{H}), 5.17(\mathrm{t}, J=9.5 \mathrm{~Hz}, 1 \mathrm{H}$, cyclobutane-CH), 3.69 (s, $3 \mathrm{H}$, ester $\left.\mathrm{CH}_{3}\right), 3.66$ (s, $3 \mathrm{H}$, ester $\left.\mathrm{CH}_{3}\right), 2.94-2.82\left(\mathrm{~m}, 1 \mathrm{H}\right.$, cyclobutane- $\left.\mathrm{CH}_{2}\right), 2.74$ (td, $J=11.0,3.1$

$\mathrm{Hz}, 1 \mathrm{H}$, cyclobutane- $\left.\mathrm{CH}_{2}\right), 2.35$ (dtd, $J=11.6,8.8,3.1 \mathrm{~Hz}, 1 \mathrm{H}$, cyclobutane- $\left.\mathrm{CH}_{2}\right), 2.18$ (dt, $J=11.7,8.9$ $\mathrm{Hz}, 1 \mathrm{H}$, cyclobutane- $\left.\mathrm{CH}_{2}\right), 1.95\left(\mathrm{~d}, J=1.2 \mathrm{~Hz}, 3 \mathrm{H}\right.$, thymine- $\left.\mathrm{CH}_{3}\right)$.

${ }^{13}$ C NMR (101 MHz, Chloroform- $d$ ) $\delta$ 170.2, 168.9, 168.7, 162.8, 150.1, 138.7, 135.0, 131.6, 130.7, $129.1,110.4,58.8,56.9,53.3,53.2,23.9,22.7,12.6$.

IR 3006 (w), 2959 (w), 2358 (w), 2257 (w), 1737 (s), 1702 (m), 1655 (s), 1438 (m), 1266 (s), 1107 (m), $984(\mathrm{w}), 914(\mathrm{w}), 733$ (s).

HRMS (ESI) calcd for $\mathrm{C}_{20} \mathrm{H}_{20} \mathrm{~N}_{2} \mathrm{NaO}_{7}{ }^{+}[\mathrm{M}+\mathrm{Na}]^{+} 423.1163$; found 423.1168 .

Dimethyl 2-(5-fluoro-2,4-dioxo-3,4-dihydropyrimidin-1(2H)-yl)cyclobutane-1,1-dicarboxylate (23)

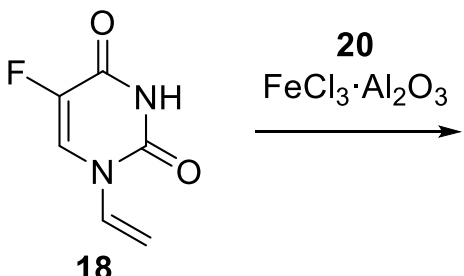

18

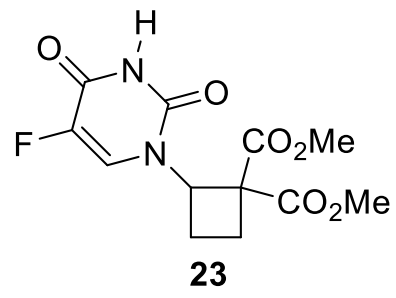

23 
Following an adapted described procedure of De Nanteuil et al., ${ }^{5}$-fluoro-1-vinylpyrimidine-2,4(1H,3H)dione (18) $(0.60 \mathrm{~g}, 3.8 \mathrm{mmol}, 1.0 \mathrm{eq})$ and iron trichloride supported on alumina $(1.0 \mathrm{mmol} / \mathrm{g}, 0.77 \mathrm{~g}, 0.77$ mmol, 0.2 eq) were added in a flame dried microwave vial under nitrogen atmosphere. Then, dry dichloromethane $(8 \mathrm{~mL})$ and a solution of crude freshly prepared dimethyl 2-methylenemalonate (20) (2.2 $\mathrm{g}, 15 \mathrm{mmol}, 4.0 \mathrm{eq})$ in dichloromethane $(2 \mathrm{~mL})$ were added into the vial and the reaction mixture was stirred at room temperature for 14 hours. The reaction mixture was filtrated over a pad of alumina eluting with ethyl acetate $(50 \mathrm{~mL})$ and concentrated under reduced pressure. The crude mixture was purified via column chromatography, eluting with a mixture of ethyl acetate/ pentane (5:5 to 2:8). The pure product 23 $(0.78 \mathrm{~g}, 2.6 \mathrm{mmol}, 67 \%$ yield) was obtained as a colorless oil.

$\mathbf{R}_{f}$ 0.28 (4:6 Pentane/Ethyl acetate).

${ }^{1}$ H NMR (400 MHz, Chloroform- $d$ ) $\delta 9.54(\mathrm{~s}, 1 \mathrm{H}, \mathrm{NH}), 7.36$ (d, $J=6.1 \mathrm{~Hz}, 1 \mathrm{H}$, F-uracil C=C-H), 5.28 $\left(\mathrm{t}, J=9.0 \mathrm{~Hz}, 1 \mathrm{H}\right.$, cyclobutane-CH), $3.78\left(\mathrm{~s}, 3 \mathrm{H}\right.$, ester $\left.\mathrm{CH}_{3}\right), 3.73\left(\mathrm{~s}, 3 \mathrm{H}\right.$, ester $\left.\mathrm{CH}_{3}\right), 2.68-2.61(\mathrm{~m}, 2$ $\mathrm{H}$, cyclobutane- $\left.\mathrm{CH}_{2}\right), 2.41-2.32\left(\mathrm{~m}, 1 \mathrm{H}\right.$, cyclobutane- $\left.\mathrm{CH}_{2}\right), 2.32-2.25\left(\mathrm{~m}, 1 \mathrm{H}\right.$, cyclobutane- $\left.\mathrm{CH}_{2}\right)$.

${ }^{13}$ C NMR (101 MHz, Chloroform- $d$ ) $\delta$ 169.7, 168.8, $156.8(\mathrm{~d}, J=26.7 \mathrm{~Hz}), 149.7,140.2(\mathrm{~d}, J=237.5$ $\mathrm{Hz}), 126.3(\mathrm{~d}, J=33.7 \mathrm{~Hz}), 59.0,55.2,53.3,53.3,23.7,23.0$.

IR 3211 (w), 3085 (w), 2960 (w), 2362 (w), 2336 (w), 1726 (s), 1439 (w), 1361 (w), 1273 (m), 1110 (w), $915(\mathrm{w}), 735(\mathrm{~m})$.

HRMS (ESI) calcd for $\mathrm{C}_{12} \mathrm{H}_{13} \mathrm{FN}_{2} \mathrm{NaO}_{6}{ }^{+}[\mathrm{M}+\mathrm{Na}]^{+} 323.0650$; found 323.0654 .

\section{Dimethyl 2-(3-benzoyl-5-fluoro-2,4-dioxo-3,4-dihydropyrimidin-1(2H)-yl)cyclobutane-1,1- dicarboxylate (4f)}<smiles>COC(=O)C1(C(C)=O)CCC1n1cc(F)c(=O)[nH]c1=O</smiles>

23

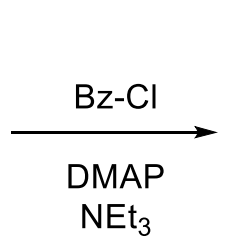

$\mathrm{NEt}_{3}$

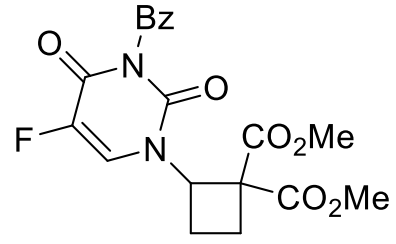

$4 f$

Following an adapted described procedure of Racine et al., ${ }^{4}$ in a flame dried microwave vial under nitrogen atmosphere, $23(0.70 \mathrm{~g}, 2.3 \mathrm{mmol}, 1.0 \mathrm{eq})$ and dimethyl-4-aminopyridine $(0.28 \mathrm{~g}, 2.3 \mathrm{mmol}, 1.0$ eq) were dissolved in dry dichloromethane $(12 \mathrm{~mL})$. Triethylamine $(1.3 \mathrm{~mL}, 9.3 \mathrm{mmol}, 4.0 \mathrm{eq})$ was added and the solution was cooled to $0^{\circ} \mathrm{C}$. Then, benzoyl chloride $(0.81 \mathrm{~mL}, 6.9 \mathrm{mmol}, 3.0 \mathrm{eq})$ was added dropwise and the reaction mixture was stirred at room temperature for 16 hours. A saturated solution of $\mathrm{NaHCO}_{3}(8 \mathrm{~mL})$ was added to the reaction mixture and the layer were separated. The aqueous layer was extracted with dichloromethane $(2 \times 20 \mathrm{~mL})$. The combined organic layers were washed with $1 \mathrm{M} \mathrm{HCl}(2$ x $40 \mathrm{~mL})$ and with water $(30 \mathrm{~mL})$, dried over $\mathrm{MgSO}_{4}$ and concentrated under reduced pressure. The pure product $4 \mathbf{f}(0.61 \mathrm{~g}, 1.5 \mathrm{mmol}, 65 \%)$ was obtained as colorless foam.

$\mathbf{R}_{f}$ 0.55 (4:6 Pentane/Ethyl acetate).

Mp 60.9- $62.5^{\circ} \mathrm{C}$.

${ }^{1}$ H NMR (400 MHz, Chloroform- $d$ ) $\delta 7.93-7.89$ (m, $2 \mathrm{H}$, Ar-H), $7.66-7.59$ (m, $\left.1 \mathrm{H}, \mathrm{Ar}-\mathrm{H}\right), 7.50-7.43$ $\left(\mathrm{m}, 3 \mathrm{H}, \mathrm{Ar}-\mathrm{H}\right.$ and F-uracil C=C-H), $5.12\left(\mathrm{t}, J=9.4 \mathrm{~Hz}, 1 \mathrm{H}\right.$, cyclobutane-CH), $3.69\left(\mathrm{~s}, 3 \mathrm{H}\right.$, ester $\left.\mathrm{CH}_{3}\right)$, $3.64\left(\mathrm{~s}, 3 \mathrm{H}\right.$, ester $\left.\mathrm{CH}_{3}\right), 2.73\left(\mathrm{q}, J=10.0 \mathrm{~Hz}, 1 \mathrm{H}\right.$, cyclobutane- $\left.\mathrm{CH}_{2}\right), 2.66-2.58(\mathrm{~m}, 1 \mathrm{H}$, cyclobutane$\left.\mathrm{CH}_{2}\right), 2.33\left(\mathrm{dtd}, J=10.6,8.4,2.4 \mathrm{~Hz}, 1 \mathrm{H}\right.$, cyclobutane- $\left.\mathrm{CH}_{2}\right), 2.23(\mathrm{dt}, J=11.6,8.9 \mathrm{~Hz}, 1 \mathrm{H}$, cyclobutane$\mathrm{CH}_{2}$ ).

${ }^{13}$ C NMR (101 MHz, Chloroform- $d$ ) $\delta 169.8,168.7,167.1,156.3(\mathrm{~d}, J=27.1 \mathrm{~Hz}), 148.6,139.8(\mathrm{~d}, J=$ $239.4 \mathrm{~Hz}), 135.5,131.0,130.9,129.3,127.1$ (d, $J=33.6 \mathrm{~Hz}), 58.7,56.8,53.4,53.3,23.9,22.8$. 
IR 3090 (w), 3009 (w), 2958 (w), 2361 (w), 2262 (w), 1723 (s), 1668 (s), 1598 (w), 1445 (m), 1357 (w), 1266 (s), 1106 (m), $982(\mathrm{w}), 912(\mathrm{~m}), 851(\mathrm{w}), 732(\mathrm{~s})$.

HRMS (ESI) calcd for $\mathrm{C}_{19} \mathrm{H}_{17} \mathrm{~N}_{2} \mathrm{FNaO}_{7}{ }^{+}[\mathrm{M}+\mathrm{Na}]^{+} 427.0917$; found 427.0920.

\subsection{Synthesis of Enol Ethers}

\section{General procedure for the synthesis of TBS enol ethers (GP1)}

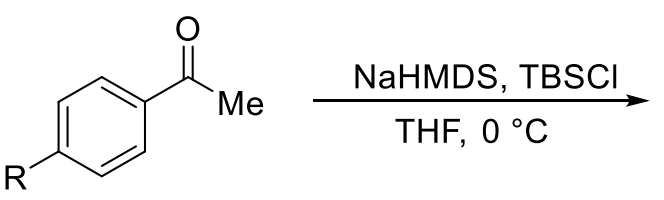

24<smiles></smiles>

7

Ketone $24(1 \mathrm{eq})$ is dissolved in dry THF $(20 \mathrm{~mL})$ under nitrogen atmosphere and cooled down to $0{ }^{\circ} \mathrm{C}$. NaHMDS (1.2 eq, 1.9 M in THF) is added dropwise. The orange solution is stirred at room temperature for 1 hour and then cooled to $0{ }^{\circ} \mathrm{C}$. The chlorosilane $(1.2 \mathrm{eq})$ is dissolved in THF $(3 \mathrm{~mL})$ and added dropwise. The reaction is stirred at room temperature for 2 hours and then concentrated under reduced pressure. The crude oil is filtered through a silica plug, eluting with $250 \mathrm{~mL}$ hexanes, concentrated under reduced pressure and purified by bulb to bulb distillation.

\section{Tert-butyldimethyl((1-phenylvinyl)oxy)silane (7a)}<smiles>C=C([OH2+])c1ccccc1</smiles>

Acetophenone (24a) $(580 \mathrm{mg}, 4.82 \mathrm{mmol}, 1 \mathrm{eq})$ in anhydrous THF $(5 \mathrm{~mL})$ is added in an oven-dried flask sealed with a septum and under $\mathrm{N}_{2}$ atmosphere. The solution is cooled down to $-78{ }^{\circ} \mathrm{C}$ and a $2 \mathrm{M}$ solution of NaHMDS (2.94 mL, $5.88 \mathrm{mmol}, 1.22 \mathrm{eq}$ ) is added dropwise. The cold bath is removed and the pale yellow solution is stirred for 1 hour at room temperature. The reaction is cooled again at $-78{ }^{\circ} \mathrm{C}$ and tertbutylchlorodimethylsilane $(871 \mathrm{mg}, 5.78 \mathrm{mmol}, 1.2 \mathrm{eq}$ ) is added dropwise. The reaction is stirred at room temperature for 5 hours after what the solvent is directly removed under reduced pressure. The resulting orange oil is purified by column chromatography on triethylamine-deactivated silica (100\% Hexane). Tert-butyldimethyl((1-phenylvinyl)oxy)silane (7a) $(960 \mathrm{mg}, 4.10 \mathrm{mmol}, 85 \%$ yield) is obtained as a colorless oil which can be re-purified by short path distillation in case of degradation with time.

${ }^{1} \mathbf{H}$ NMR $\left(400 \mathrm{MHz}, \mathrm{CDCl}_{3}\right) \delta$ 7.65-7.60 (m, $\left.2 \mathrm{H}, \mathrm{Ar}\right), 7.39-7.29(\mathrm{~m}, 3 \mathrm{H}, \mathrm{Ar}), 4.89(\mathrm{~d}, 1 \mathrm{H}, J=1.7 \mathrm{~Hz}$, $\left.\mathrm{C}=\mathrm{CH}_{2}\right), 4.42\left(\mathrm{~d}, 1 \mathrm{H}, J=1.7 \mathrm{~Hz}, \mathrm{C}=\mathrm{CH}_{2}\right), 1.00\left(\mathrm{~s}, 9 \mathrm{H}, \mathrm{Si}\left(\mathrm{CH}_{3}\right)_{2} \mathrm{C}\left(\mathrm{CH}_{3}\right)_{3}\right), 0.21 \quad(\mathrm{~s}, 6 \mathrm{H}$, $\left.\mathrm{Si}\left(\mathrm{CH}_{3}\right)_{2} \mathrm{C}\left(\mathrm{CH}_{3}\right)_{3}\right)$.

${ }^{13} \mathbf{C}$ NMR $\left(101 \mathrm{MHz}, \mathrm{CDCl}_{3}\right) \delta 156.0,137.8,128.2,128.1,125.3,90.9,25.9,18.4,-4.6$.

Data match literature report. ${ }^{7}$

Tert-butyl(1-(4-methoxyphenyl)vinyl)oxy)dimethylsilane (7b)

${ }^{7}$ J.-F. Zhao, B.-H. Tan, T.-P. Loh Chem. Sci. 2011, 2, 349. 


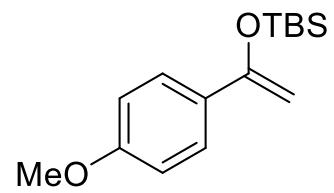

Following GP1, 1-(4-methoxyphenyl)ethanone (24b) (2.0 g, 13 mmol, 1 eq), NaHMDS (8.4 mL, 16 mmol, 1.2 eq and tert-butylchlorodimethylsilane $(2.4 \mathrm{~g}, 16 \mathrm{mmol}, 1.2 \mathrm{eq})$ were reacted. Tert-butyl(1-(4methoxyphenyl)vinyl)oxy)dimethylsilane (7b) $(2.5 \mathrm{~g}, 9.5 \mathrm{mmol}, 71 \%)$ was obtained as a colorless oil.

${ }^{1}$ H NMR $\left(400 \mathrm{MHz}, \mathrm{DMSO}-d_{6}\right) \delta 7.54(\mathrm{~d}, J=8.9 \mathrm{~Hz}, 2 \mathrm{H}, \mathrm{Ar}), 6.93(\mathrm{~d}, J=8.9 \mathrm{~Hz}, 2 \mathrm{H}, \mathrm{Ar}), 4.89(\mathrm{~d}, J=$ $1.8 \mathrm{~Hz}, 1 \mathrm{H}, \mathrm{CH}_{2}$ vinyl), 4.32 (d, $J=1.8 \mathrm{~Hz}, 1 \mathrm{H}, \mathrm{CH}_{2}$ vinyl), 3.77 (s, $3 \mathrm{H}, \mathrm{OMe}$ ), 0.98 (s, $9 \mathrm{H}, \mathrm{OTBS}$ ), 0.20 (s, $6 \mathrm{H}, \mathrm{OTBS})$.

${ }^{13}$ C NMR (101 MHz, DMSO) $\delta$ 159.4, 154.7, 129.5, 126.2, 113.5, 89.4, 55.1, 25.7, 18.0, -4.7.

NMR data of $\mathbf{7 b}$ corresponded to the reported spectra. ${ }^{8}$

\section{Tert-butyl(1-(4-fluorophenyl)vinyl)oxy)dimethylsilane (7c)}

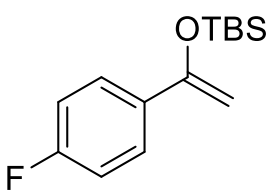

Following GP1, 1-(4-fluorophenyl)ethanone (24c) (2.0 g, 15 mmol, 1 eq), NaHMDS (9.1 mL, 17 mmol, $1.2 \mathrm{eq}$ and tert-butylchlorodimethylsilane $(2.6 \mathrm{~g}, 17 \mathrm{mmol}, 1.2 \mathrm{eq})$ were reacted. Tert-butyl(1-(4fluorophenyl)vinyl)oxy)dimethylsilane (7c) $(0.80 \mathrm{~g}, 3.2 \mathrm{mmol}, 22 \%)$ was obtained as a colorless oil.

${ }^{1} \mathbf{H}$ NMR $\left(400 \mathrm{MHz}\right.$, DMSO- $\left.d_{6}\right) \delta 7.65(\mathrm{dd}, J=9.0,5.5 \mathrm{~Hz}, 2 \mathrm{H}, \mathrm{Ar}), 7.21(\mathrm{t}, J=8.9 \mathrm{~Hz}, 2 \mathrm{H}, \mathrm{Ar}), 5.00$ (d, $J=2.1 \mathrm{~Hz}, 1 \mathrm{H}, \mathrm{CH}_{2}$ vinyl), $4.43\left(\mathrm{~d}, J=2.0 \mathrm{~Hz}, 1 \mathrm{H}, \mathrm{CH}_{2}\right.$ vinyl), $0.98(\mathrm{~d}, J=0.8 \mathrm{~Hz}, 9 \mathrm{H}, \mathrm{OTBS})$, $0.21(\mathrm{~d}, J=0.9 \mathrm{~Hz}, 6 \mathrm{H}$, OTBS$)$.

${ }^{13}$ C NMR (101 MHz, DMSO) $\delta 162.1(\mathrm{~d}, J=245.5 \mathrm{~Hz}), 153.9,133.5(\mathrm{~d}, J=3.2 \mathrm{~Hz}), 126.9(\mathrm{~d}, J=8.4$ $\mathrm{Hz}), 115.1(\mathrm{~d}, J=21.6 \mathrm{~Hz}), 91.1,25.6,17.9,-4.8$.

IR 2891 (w), 2866 (w), $2821(\mathrm{w}), 2788$ (w), 1564 (w), 1470 (w), 1456 (m), 1456 (m), 1351 (m), $1311(\mathrm{w})$, 1144 (s), 1144 (s), 1122 (s), 1085 (m), 1060 (s), 1060 (s), 823 (s).

HRMS (ESI) calcd for $\mathrm{C}_{14} \mathrm{FH}_{21} \mathrm{KOSi}^{+}[\mathrm{M}+\mathrm{K}]^{+}$291.0977; found 291.1180.

\section{Methyl 4-(1-(tert-butyldimethylsilyl)oxy)vinyl)benzoate (7d)}<smiles>C=C([O+])OC(=C)c1ccc(C(C)=O)cc1</smiles>

Following GP1, methyl 4-acetylbenzoate (24d) (1.0 g, 5.6 mmol, 1 eq), NaHMDS (3.5 mL, 6.7 mmol, 1.2 eq and tert-butylchlorodimethylsilane $(1.0 \mathrm{~g}, 6.7 \mathrm{mmol}, 1.2 \mathrm{eq})$ were reacted. Methyl 4-(1-(tertbutyldimethylsilyl)oxy)vinyl)benzoate (7d) $(0.50 \mathrm{~g}, 1.7 \mathrm{mmol}, 31 \%)$ was obtained as a colorless oil.

${ }^{1} \mathbf{H}$ NMR $\left(400 \mathrm{MHz}, \mathrm{DMSO}-d_{6}\right) \delta 8.07-7.86(\mathrm{~m}, 2 \mathrm{H}, \mathrm{Ar}), 7.86-7.68(\mathrm{~m}, 2 \mathrm{H}, \mathrm{Ar}), 5.20(\mathrm{~d}, J=2.2 \mathrm{~Hz}$, $1 \mathrm{H}, \mathrm{CH}_{2}$ vinyl), 4.59 (d, $J=2.2 \mathrm{~Hz}, 1 \mathrm{H}, \mathrm{CH}_{2}$ vinyl), 3.86 (s, $3 \mathrm{H}, \mathrm{OMe}$ ), 0.98 (s, $9 \mathrm{H}, \mathrm{OTBS}$ ), 0.22 (s, 6 H, OTBS).

\footnotetext{
${ }^{8}$ Ventura, D. L.; Li, Z.; Coleman, M. G.; Davies, H. M. L. Tetrahedron 2009, 65, 3052.
} 
${ }^{13}$ C NMR (101 MHz, DMSO) $\delta$ 165.8, 153.9, 141.4, 129.2, 125.0, 93.8, 52.1, 30.7, 25.6, 18.0, -4.8.

IR 2888 (w), 2866 (w), 2821 (w), 2820 (w), 2787 (w), 1583 (s), 1464 (w), 1146 (m), 1106 (s), 937 (m), $926(\mathrm{~m}), 828(\mathrm{~m})$.

HRMS (ESI) calcd for $\mathrm{C}_{16} \mathrm{H}_{25} \mathrm{O}_{3} \mathrm{Si}^{+}[\mathrm{M}+\mathrm{H}]^{+} 293.1567$; found 293.1560.

\section{(Z)-Tert-butyldimethyl((1-phenylprop-1-en-1-yl)oxy)silane (7e)}<smiles>CC=C(O[Sb])c1ccccc1</smiles>

Following GP1, propiophenone (24e) $(1.0 \mathrm{~g}, 7.5 \mathrm{mmol}, 1 \mathrm{eq})$, NaHMDS (4.7 mL, $8.9 \mathrm{mmol}, 1.2 \mathrm{eq})$ and tert-butylchlorodimethylsilane $(1.3 \mathrm{~g}, 8.9 \mathrm{mmol}, 1.2 \mathrm{eq})$ were reacted. (Z)-Tert-butyldimethyl((1phenylprop-1-en-1-yl)oxy)silane (7e) $(0.51 \mathrm{~g}, 2.0 \mathrm{mmol}, 27 \%)$ was obtained as a colorless oil, which was used directly in the [4+2] annulation step.

\section{Optimization of the $[4+2]$ Annulation}

\section{Optimization procedure for $\mathrm{SnCl}_{4}$ and $\mathrm{TiCl}_{4}$ :}

Preparation of the $\mathrm{SnCl}_{4}$ solution: in an oven dried flask, under nitrogen atmosphere, $\mathrm{SnCl}_{4}(0.22 \mathrm{~g}, 0.10$ $\mathrm{mL}, 0.86 \mathrm{mmol})$ were added to dry dichloromethane $(1.9 \mathrm{~mL})$ to make a $0.43 \mathrm{M}$ solution.

Preparation of the $\mathrm{TiCl}_{4}$ solution: in an oven dried flask, under nitrogen atmosphere, $\mathrm{TiCl}_{4}(0.17 \mathrm{~g}, 0.10$ $\mathrm{mL}, 0.91 \mathrm{mmol})$ were added to dry dichloromethane $(1.9 \mathrm{~mL})$ to make a $0.46 \mathrm{M}$ solution.

The cyclobutane $4(0.050 \mathrm{mmol}, 1 \mathrm{eq})$ was dissolved in dry dichloromethane $(1.0 \mathrm{~mL})$, and added in an oven dried microwave vial under nitrogen atmosphere. The aldehyde 5a $(0.075 \mathrm{mmol}, 1.5 \mathrm{eq})$ was then dissolved in dry dichloromethane $(0.50 \mathrm{~mL})$ and added. The Lewis acid solution was then added dropwise to the reaction mixture. The reaction was quenched with triethylamine $(0.10 \mathrm{~mL})$, and concentrated under vacuum. The crude mixture was then dissolved in $\mathrm{CDCl}_{3}$ and analyzed by ${ }^{1} \mathrm{H} \mathrm{NMR}$.

\section{Optimization procedure for other Lewis acids:}

In the glovebox, the Lewis acid was added to a microwave vial. The vial was sealed with a Teflon septum and taken out of the glovebox. Dry dichloromethane $(0.50 \mathrm{~mL})$ was added. The cyclobutane $4(0.050$ mmol, 1 eq) was dissolved in dry dichloromethane $(0.50 \mathrm{~mL})$ and added. The aldehyde $\mathbf{5 a}(0.075 \mathrm{mmol}$, $1.5 \mathrm{eq})$ was then dissolved in dry dichloromethane $(0.50 \mathrm{~mL})$ and added. The reaction was then filtered over a pad of alumina, eluted with ethyl acetate and concentrated under vacuum. The crude mixture was then dissolved in $\mathrm{CDCl}_{3}$ and analyzed by ${ }^{1} \mathrm{H} \mathrm{NMR}$.

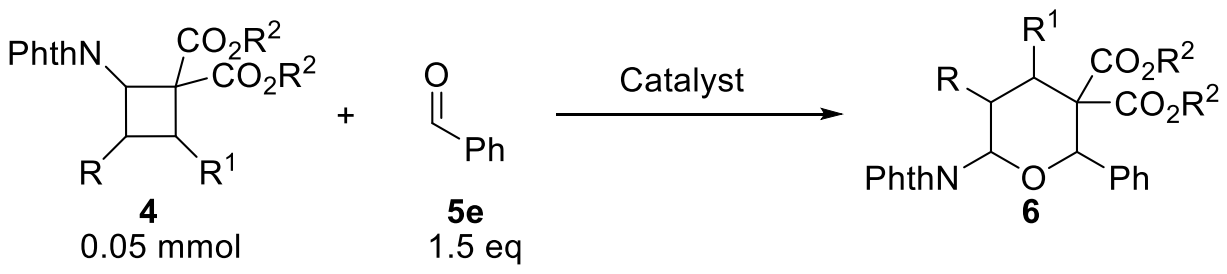




\begin{tabular}{|c|c|c|c|c|c|c|c|}
\hline Entry & Catalyst (mol\%) & Time & $\mathbf{T}$ & Solvent & Conversion $^{\mathrm{a}}$ & $d r^{b}$ & $\mathbf{R}, \mathbf{R}^{1}, \mathbf{R}^{2}$ \\
\hline 1 & $\mathrm{FeCl}_{3} \cdot \mathrm{Al}_{2} \mathrm{O}_{3}(20 \mathrm{~mol} \%)$ & $40 \mathrm{~min}$ & $\mathrm{rt}$ & $\mathrm{DCM}$ & $>95 \%^{\mathrm{c}}$ & $2: 1$ & $\mathrm{H}, \mathrm{H}, \mathrm{Me}$ \\
\hline 2 & $\mathrm{SnCl}_{4}\left(20 \mathrm{~mol}^{2}\right)$ & $2.5 \mathrm{~h}$ & $\mathrm{rt}$ & DCM & $>95 \%{ }^{\mathrm{c}}$ & $6: 1$ & $\mathrm{H}, \mathrm{H}, \mathrm{Me}$ \\
\hline 3 & $\mathrm{Hf}(\mathrm{OTf})_{4}(20 \mathrm{~mol} \%)$ & $2.5 \mathrm{~h}$ & $\mathrm{rt}$ & DCM & $>95 \%{ }^{\mathrm{c}}$ & $9: 1$ & $\mathrm{H}, \mathrm{H}, \mathrm{Me}$ \\
\hline 4 & $\mathrm{Yb}(\mathrm{OTf})_{3}(20 \mathrm{~mol} \%)$ & $2.5 \mathrm{~h}$ & $\mathrm{rt}$ & $\mathrm{DCM}$ & $<5 \%{ }^{\mathrm{d}}$ & - & $\mathrm{H}, \mathrm{H}, \mathrm{Me}$ \\
\hline 5 & $\mathrm{Sn}(\mathrm{OTf})_{2}(20 \mathrm{~mol} \%)$ & $2.5 \mathrm{~h}$ & $\mathrm{rt}$ & DCM & $<5 \%$ & - & $\mathrm{H}, \mathrm{H}, \mathrm{Me}$ \\
\hline 6 & $\mathrm{Cu}(\mathrm{OTf})_{2}(20 \mathrm{~mol} \%)$ & $2.5 \mathrm{~h}$ & $\mathrm{rt}$ & DCM & $<5 \%$ & - & $\mathrm{H}, \mathrm{H}, \mathrm{Me}$ \\
\hline 7 & $\mathrm{AuCl}(20 \mathrm{~mol} \%)$ & $2.5 \mathrm{~h}$ & $\mathrm{rt}$ & DCM & $<5 \%$ & - & $\mathrm{H}, \mathrm{H}, \mathrm{Me}$ \\
\hline 8 & $\mathrm{AuCl}_{3}(20 \mathrm{~mol} \%)$ & $2.5 \mathrm{~h}$ & $\mathrm{rt}$ & $\mathrm{DCM}$ & $>95 \%{ }^{\mathrm{e}}$ & - & $\mathrm{H}, \mathrm{H}, \mathrm{Me}$ \\
\hline 9 & $\mathrm{TiCl}_{4}(20 \mathrm{~mol} \%)$ & $2.5 \mathrm{~h}$ & $\mathrm{rt}$ & DCM & $88 \%^{\mathrm{c}}$ & $5: 1$ & $\mathrm{H}, \mathrm{H}, \mathrm{Me}$ \\
\hline 10 & $\operatorname{In}(\mathrm{OTf})_{3}(20 \mathrm{~mol} \%)$ & $2.5 \mathrm{~h}$ & $\mathrm{rt}$ & DCM & $>95 \%$ & $9: 1$ & $\mathrm{H}, \mathrm{H}, \mathrm{Me}$ \\
\hline 11 & $\mathrm{Sc}(\mathrm{OTf})_{3}(20 \mathrm{~mol} \%)$ & $2.5 \mathrm{~h}$ & $\mathrm{rt}$ & DCM & $>95 \%$ & $13: 1$ & $\mathrm{H}, \mathrm{H}, \mathrm{Me}$ \\
\hline 12 & $\mathrm{Sc}(\mathrm{OTf})_{3}(20 \mathrm{~mol} \%)$ & $7 \mathrm{~h}$ & reflux & $\mathrm{DCM}$ & $<5 \%{ }^{\mathrm{d}}$ & - & $\mathrm{Me}, \mathrm{H}, \mathrm{Me}$ \\
\hline 13 & $\operatorname{In}(\mathrm{OTf})_{3}(20 \mathrm{~mol} \%)$ & $18 \mathrm{~h}$ & reflux & DCM & $34 \%$ & $\begin{array}{l}4.5: 1 \\
: 1\end{array}$ & $\mathrm{Me}, \mathrm{H}, \mathrm{Me}$ \\
\hline 14 & $\mathrm{FeCl}_{3}(100 \mathrm{~mol} \%)$ & $18 \mathrm{~h}$ & $\mathrm{rt}$ & DCM & $>95 \%$ & $\begin{array}{l}6: 1: 1 \\
f\end{array}$ & $\mathrm{Me}, \mathrm{H}, \mathrm{Me}$ \\
\hline 15 & $\mathrm{SnCl}_{4}(20 \mathrm{~mol} \%)$ & $2.5 \mathrm{~h}$ & $\mathrm{rt}$ & $\mathrm{DCM}$ & $<5 \%{ }^{\mathrm{d}}$ & - & $\mathrm{Me}, \mathrm{H}, \mathrm{Me}$ \\
\hline 16 & $\mathrm{SnCl}_{4}(100 \mathrm{~mol} \%)$ & $2.5 \mathrm{~h}$ & $-40^{\circ} \mathrm{C}$ & DCM & $>95 \%{ }^{\mathrm{e}}$ & - & $\mathrm{Me}, \mathrm{H}, \mathrm{Me}$ \\
\hline 17 & $\mathrm{FeCl}_{3} \cdot \mathrm{SiO}_{2}(100 \mathrm{~mol} \%)$ & $18 \mathrm{~h}$ & $\mathrm{rt}$ & DCM & $>95 \% \mathrm{c}$ & - & $\mathrm{Me}, \mathrm{H}, \mathrm{Me}$ \\
\hline 18 & $\mathrm{FeBr}_{3}(100 \mathrm{~mol} \%)$ & $18 \mathrm{~h}$ & $\mathrm{rt}$ & DCM & $>95 \%^{\mathrm{c}}$ & - & $\mathrm{Me}, \mathrm{H}, \mathrm{Me}$ \\
\hline 19 & $\mathrm{FeCl}_{3} \cdot \mathrm{Al}_{2} \mathrm{O}_{3}(100 \mathrm{~mol} \%)$ & $2.5 \mathrm{~h}$ & $\mathrm{rt}$ & $\mathrm{ACN}$ & $<5 \%{ }^{\mathrm{d}}$ & - & $\mathrm{Me}, \mathrm{H}, \mathrm{Me}$ \\
\hline 20 & $\mathrm{FeCl}_{3} \cdot \mathrm{Al}_{2} \mathrm{O}_{3}(100 \mathrm{~mol} \%)$ & $2.5 \mathrm{~h}$ & $\mathrm{rt}$ & $\mathrm{MeOH}$ & $<5 \%{ }^{\mathrm{d}}$ & - & $\mathrm{Me}, \mathrm{H}, \mathrm{Me}$ \\
\hline 21 & $\mathrm{FeCl}_{3} \cdot \mathrm{Al}_{2} \mathrm{O}_{3}(100 \mathrm{~mol} \%)$ & $2.5 \mathrm{~h}$ & $\mathrm{rt}$ & $\overline{\mathrm{DMF}}$ & $<5 \%{ }^{\mathrm{d}}$ & - & $\mathrm{Me}, \mathrm{H}, \mathrm{Me}$ \\
\hline 22 & $\mathrm{FeCl}_{3} \cdot \mathrm{Al}_{2} \mathrm{O}_{3}(100 \mathrm{~mol} \%)$ & $2.5 \mathrm{~h}$ & $\mathrm{rt}$ & THF & $<5 \%{ }^{\mathrm{d}}$ & - & $\mathrm{Me}, \mathrm{H}, \mathrm{Me}$ \\
\hline 23 & $\mathrm{FeCl}_{3} \cdot \mathrm{Al}_{2} \mathrm{O}_{3}(100 \mathrm{~mol} \%)$ & $18 \mathrm{~h}$ & $\mathrm{rt}$ & Toluene & $76 \%{ }^{\mathrm{c}}$ & - & $\mathrm{Me}, \mathrm{H}, \mathrm{Me}$ \\
\hline 24 & $\mathrm{FeCl}_{3} \cdot \mathrm{Al}_{2} \mathrm{O}_{3}(100 \mathrm{~mol} \%)^{\mathrm{g}}$ & $5 \mathrm{~h}$ & $\mathrm{rt}$ & $\mathrm{DCM}$ & $>95 \%{ }^{\mathrm{e}}$ & - & $\mathrm{Me}, \mathrm{H}, \mathrm{Me}$ \\
\hline 25 & $\mathrm{FeCl}_{3} \cdot \mathrm{Al}_{2} \mathrm{O}_{3}(100 \mathrm{~mol} \%)$ & $5 \mathrm{~h}$ & $\mathrm{rt}$ & $\mathrm{DCM}$ & $>95 \%$ & $5: 1$ & $\mathrm{Me}, \mathrm{H}, \mathrm{Me}$ \\
\hline 26 & $\mathrm{InOTf}_{3}(20 \mathrm{~mol} \%)$ & $5 \mathrm{~h}$ & reflux & DCM & $77 \%$ & $5: 1$ & $\mathrm{H}, \mathrm{Me}, \mathrm{Et}$ \\
\hline 27 & $\mathrm{AlCl}_{3}(20 \mathrm{~mol} \%)$ & $24 \mathrm{~h}$ & reflux & $\mathrm{DCM}$ & $45 \%{ }^{\mathrm{c}}$ & $4: 1$ & $\mathrm{H}, \mathrm{Me}, \mathrm{Et}$ \\
\hline
\end{tabular}




\begin{tabular}{|l|l|l|l|l|l|l|l|}
\hline 28 & $\mathrm{TfOH}(20 \mathrm{~mol} \%)$ & $18 \mathrm{~h}$ & reflux & DCM & $61 \%^{\mathrm{c}}$ & $1.7: 1$ & $\mathrm{H}, \mathrm{Me}, \mathrm{Et}$ \\
\hline 29 & $\mathrm{FeCl}_{3} \cdot \mathrm{Al}_{2} \mathrm{O}_{3}(20 \mathrm{~mol} \%)$ & $30 \mathrm{~h}$ & reflux & DCM & $89 \%^{\mathrm{c}}$ & $3: 1$ & $\mathrm{H}, \mathrm{Me}, \mathrm{Et}$ \\
\hline 30 & $\mathrm{FeCl}_{3} \cdot \mathrm{Al}_{2} \mathrm{O}_{3}(20 \mathrm{~mol} \%)$ & $1 \mathrm{~h}$ & reflux & DCE & $>95 \%^{\mathrm{e}}$ & - & $\mathrm{H}, \mathrm{Me}, \mathrm{Et}$ \\
\hline 31 & $\mathrm{FeCl}_{3} \cdot \mathrm{Al}_{2} \mathrm{O}_{3}(20 \mathrm{~mol} \%)^{\mathrm{h}}$ & $2.5 \mathrm{~h}$ & $\mathrm{rt}$ & $\mathrm{DCM}$ & $86 \%^{\mathrm{c}}$ & $3: 1$ & $\mathrm{H}, \mathrm{Me}, \mathrm{Et}$ \\
\hline 33 & $\mathrm{FeCl}_{3} \cdot \mathrm{Al}_{2} \mathrm{O}_{3}(100 \mathrm{~mol} \%)$ & $2.5 \mathrm{~h}$ & $\mathrm{rt}$ & $\mathrm{DCM}$ & $>95 \%^{\mathrm{c}}$ & $1.2: 1$ & $\mathrm{H}, \mathrm{Me}, \mathrm{Et}$ \\
\hline 34 & $\mathrm{FeCl}_{3} \cdot \mathrm{Al}_{2} \mathrm{O}_{3}(100 \mathrm{~mol} \%)$ & $18 \mathrm{~h}$ & $-30{ }^{\circ} \mathrm{C}$ & $\mathrm{DCM}$ & $<5 \%^{\mathrm{d}}$ & - & $\mathrm{H}, \mathrm{Me}, \mathrm{Et}$ \\
\hline 35 & $\mathrm{FeCl}_{3} \cdot \mathrm{Al}_{2} \mathrm{O}_{3}(100 \mathrm{~mol} \%)$ & $20 \mathrm{~h}$ & $-20^{\circ} \mathrm{C}$ & $\mathrm{DCM}$ & $>95 \%$ & $4: 1$ & $\mathrm{H}, \mathrm{Me}, \mathrm{Et}$ \\
\hline 36 & $\mathrm{FeCl}_{3} \cdot \mathrm{Al}_{2} \mathrm{O}_{3}(50 \mathrm{~mol} \%)$ & $5.5 \mathrm{~h}$ & $0{ }^{\circ} \mathrm{C}$ & $\mathrm{DCM}$ & $90 \%$ & $4: 1$ & $\mathrm{H}, \mathrm{Me}, \mathrm{Et}$ \\
\hline 37 & $\mathrm{FeCl}_{3} \cdot \mathrm{Al}_{2} \mathrm{O}_{3}(100 \mathrm{~mol} \%)$ & $18 \mathrm{~h}$ & $\mathrm{rt}$ & $\mathrm{DCM}$ & $>95 \%$ & $5: 1$ & $\mathrm{Ph}, \mathrm{H}, \mathrm{Me}$ \\
\hline 38 & $\mathrm{ScOTf}_{3}(20 \mathrm{~mol} \%)$ & $4 \mathrm{~h}$ & $\mathrm{rt}$ & $\mathrm{DCM}$ & $<5 \%^{\mathrm{d}}$ & - & $\mathrm{Ph}, \mathrm{H}, \mathrm{Me}$ \\
\hline 39 & $\mathrm{YbOTf}_{3}(20 \mathrm{~mol} \%)$ & $4 \mathrm{~h}$ & $\mathrm{rt}$ & $\mathrm{DCM}$ & $<5 \%^{\mathrm{d}}$ & - & $\mathrm{Ph}, \mathrm{H}, \mathrm{Me}$ \\
\hline 40 & $\mathrm{SnCl}_{4}(100 \mathrm{~mol} \%)$ & $4 \mathrm{~h}$ & $\mathrm{rt}$ & $\mathrm{DCM}$ & $<5 \%^{\mathrm{d}}$ & - & $\mathrm{Ph}, \mathrm{H}, \mathrm{Me}$ \\
\hline 41 & $\mathrm{FeCl}_{3}(100 \mathrm{~mol} \%)$ & $18 \mathrm{~h}$ & $\mathrm{rt}$ & $\mathrm{DCM}$ & $>95 \%^{\mathrm{c}}$ & - & $\mathrm{Ph}, \mathrm{H}, \mathrm{Me}$ \\
\hline 42 & $\mathrm{FeBr}_{3}(100 \mathrm{~mol} \%)$ & $18 \mathrm{~h}$ & $\mathrm{rt}$ & $\mathrm{DCM}$ & $>95 \%^{\mathrm{c}}$ & - & $\mathrm{Ph}, \mathrm{H}, \mathrm{Me}$ \\
\hline
\end{tabular}

${ }^{a}$ Estimated by ${ }^{1} \mathrm{H}$ NMR of the crude mixture based on the ratio of product to starting material; ${ }^{b}$ Determined by ${ }^{1} \mathrm{H}$ NMR of the crude; ${ }^{\mathrm{c}}$ Complex mixture of products was observed in the ${ }^{1} \mathrm{H}$ NMR of the crude; ${ }^{\mathrm{d}}$ No product detected; ${ }^{\mathrm{e}}$ Degradation; ${ }^{\mathrm{f}}$ Not reproducible; ${ }^{\mathrm{g}}$ Open flask; ${ }^{\mathrm{h}} 3$ equivalents of aldehyde.

\section{Optimization procedure for cyclobutane $4 \mathrm{e}$ and $4 \mathrm{f}$}

The Lewis acid was added in a flame dried microwave vial and under nitrogen atmosphere. Then, dry dichloromethane and benzaldehyde $(\mathbf{5 a})(7.7 \mu \mathrm{L}, 0.076 \mathrm{mmol}, 1.5 \mathrm{eq})$ were added into the flask. A solution of cyclobutane $4(20 \mathrm{mg}, 0.050 \mathrm{mmol}, 1.0 \mathrm{eq})$ in dry dichloromethane was added dropwise to the reaction mixture. The reaction mixture was filtrated on a pad of silica eluting with ethyl acetate $(10 \mathrm{~mL})$ and concentrated under reduce pressure. The crude product was dissolved in ethanol $(1.8 \mathrm{~mL})$ and ammonium hydroxide solution $(0.6 \mathrm{~mL}, 60 \mathrm{eq}, 25 \%)$ was added. The resulting solution was stirred for 16 hours and the solvent was removed under reduced pressure. The crude mixture was purified by column chromatography eluting with a mixture of ethyl acetate/ pentane (5:5 to 9:1).

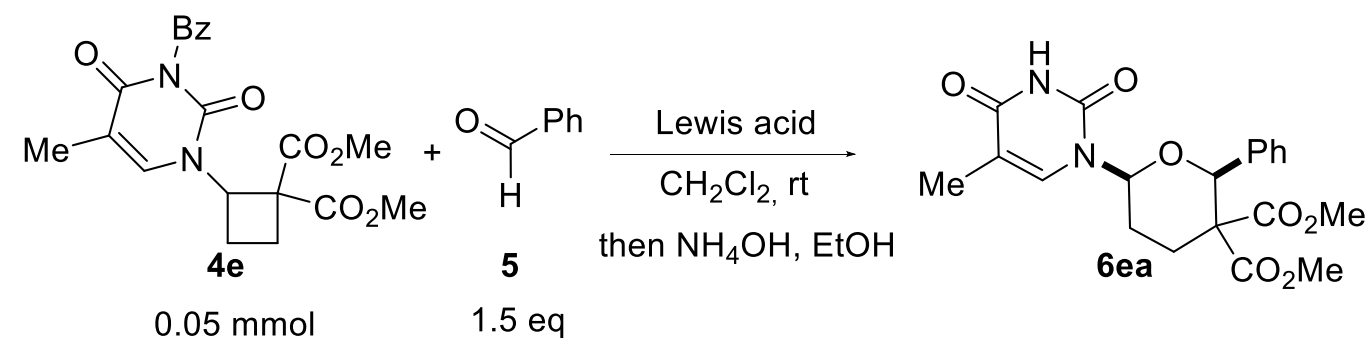




\begin{tabular}{|l|l|l|l|l|}
\hline Entry & Catalyst (mol\%) & Time & Conversion $^{\mathbf{a}}$ & Yield $^{\mathbf{b}}$ \\
\hline 1 & $\mathrm{Hf}(\mathrm{OTf})_{4}(20 \mathrm{~mol} \%)$ & $14 \mathrm{~h}$ & $>95 \%$ & $51 \%$ \\
\hline 2 & $\mathrm{FeCl}_{3} \cdot \mathrm{Al}_{2} \mathrm{O}_{3}(20 \mathrm{~mol} \%)$ & $14 \mathrm{~h}$ & $>95 \%$ & $59 \%$ \\
\hline 3 & $\mathrm{Hf}(\mathrm{OTf})_{4}(20 \mathrm{~mol} \%)$ & $30 \mathrm{~min}$ & $>95 \%$ & $76 \%$ \\
\hline 4 & $\mathrm{FeCl}_{3} \cdot \mathrm{Al}_{2} \mathrm{O}_{3}(100 \mathrm{~mol} \%)$ & $30 \mathrm{~min}$ & $>95 \%$ & $62 \%$ \\
\hline 5 & $\mathrm{Hf}(\mathrm{OTf})_{4}(20 \mathrm{~mol} \%)$ & $15 \mathrm{~min}$ & $>95 \%$ & $84 \%$ \\
\hline 6 & $\mathrm{FeCl}_{3} \cdot \mathrm{Al}_{2} \mathrm{O}_{3}(100 \mathrm{~mol} \%)$ & $15 \mathrm{~min}$ & $>95 \%$ & $70 \%$ \\
\hline 7 & $\mathrm{Hf}(\mathrm{OTf})_{4}(10 \mathrm{~mol} \%)$ & $15 \mathrm{~min}$ & $>95 \%$ & $87 \%$ \\
\hline
\end{tabular}

${ }^{\mathrm{a}}$ Estimated by ${ }^{1} \mathrm{H}$ NMR of the crude mixture based on the ratio of product to starting material, ${ }^{\mathrm{b}}{ }^{1} \mathrm{H} \mathrm{NMR}$ yield (traces of benzamide originated from the protecting group) 


\section{Scope of the [4+2] Annulation}

\subsection{General Procedures}

General Procedure for the [4+2] annulation reaction of dimethyl 2-(1,3-dioxoisoindolin-2yl)cyclobutane-1,1-dicarboxylate (4a). (GP2)

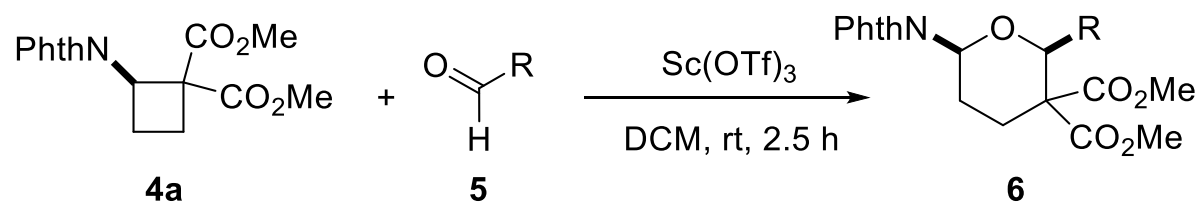

In a glovebox, scandium triflate $(19.7 \mathrm{mg}, 0.0400 \mathrm{mmol}, 0.2 \mathrm{eq})$ was added to a microwave vial. The vial was sealed with a Teflon septum and taken out of the glovebox. Dry dichloromethane $(2 \mathrm{~mL})$ was added. The cyclobutane 4a $(0.200 \mathrm{mmol}, 1 \mathrm{eq})$ was dissolved in dry dichloromethane $(2 \mathrm{~mL})$ and added. The aldehyde 5 (0.300 mmol, $1.5 \mathrm{eq})$ was then dissolved in dry dichloromethane $(2 \mathrm{~mL})$ and added. The reaction was stirred at room temperature for $2.5 \mathrm{~h}$, and then filtered over a pad of basic alumina, eluted with ethyl acetate and concentrated under vacuum. The crude product was purified by column chromatography using the indicated solvents.

General Procedure for the [4+2] annulation reaction of dimethyl 2-(1,3-dioxoisoindolin-2-yl)-3methylcyclobutane-1,1-dicarboxylate (4b). (GP3)

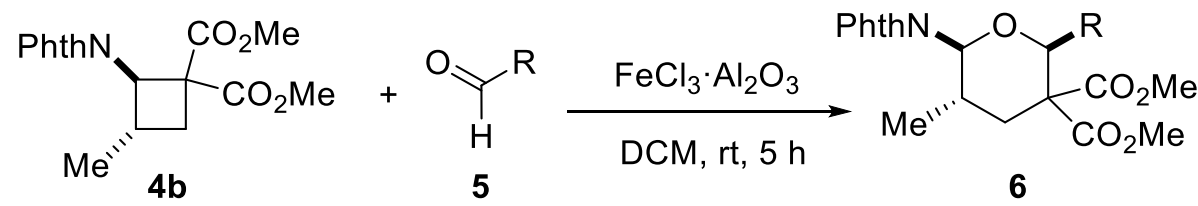

In a glovebox, iron trichloride supported on alumina $(1.00 \mathrm{mmol} / \mathrm{g}, 200 \mathrm{mg}, 0.200 \mathrm{mmol}, 1 \mathrm{eq})$ was added to a microwave vial. The vial was sealed with a Teflon septum and taken out of the glovebox. Dry dichloromethane $(2 \mathrm{~mL})$ was added. The cyclobutane $\mathbf{4 b}(0.200 \mathrm{mmol}, 1 \mathrm{eq})$ was dissolved in dry dichloromethane $(2 \mathrm{~mL})$ and added. The aldehyde $5(0.300 \mathrm{mmol}, 1.5 \mathrm{eq})$ was then dissolved in dry dichloromethane $(2 \mathrm{~mL})$ and added. The reaction was stirred at room temperature for $5 \mathrm{~h}$, and then filtered over a pad of basic alumina, eluted with ethyl acetate and concentrated under vacuum. The crude product was purified by column chromatography using the indicated solvents.

General Procedure for the [4+2] annulation reaction of diethyl 2-(1,3-dioxoisoindolin-2-yl)-4methylcyclobutane-1,1-dicarboxylate (4d). (GP4)

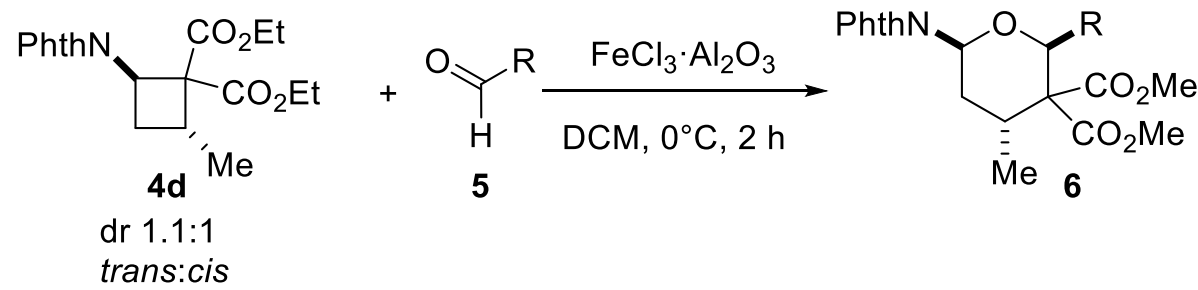

In a glovebox, iron trichloride supported on alumina $(1.00 \mathrm{mmol} / \mathrm{g}, 200 \mathrm{mg}, 0.200 \mathrm{mmol}, 1 \mathrm{eq})$ was added to a microwave vial. The vial was sealed with a Teflon septum and taken out of the glovebox. Dry dichloromethane $(2 \mathrm{~mL})$ was added. The vial was cooled down at $0{ }^{\circ} \mathrm{C}$ with an ice/water bath. The cyclobutane 4d $(0.200 \mathrm{mmol}, 1 \mathrm{eq})$ was dissolved in dry dichloromethane $(2 \mathrm{~mL})$ and added dropwise. 
The aldehyde $5(0.300 \mathrm{mmol}, 1.5 \mathrm{eq})$ was then dissolved in dry dichloromethane $(2 \mathrm{~mL})$ and added dropwise. The reaction was stirred at $0{ }^{\circ} \mathrm{C}$ for $2 \mathrm{~h}$, and then filtered over a pad of basic alumina, eluted with ethyl acetate and concentrated under vacuum. The crude product was purified by column chromatography using the indicated solvents.

General Procedure for the [4+2] annulation for the synthesis of nucleoside analogues (GP5)

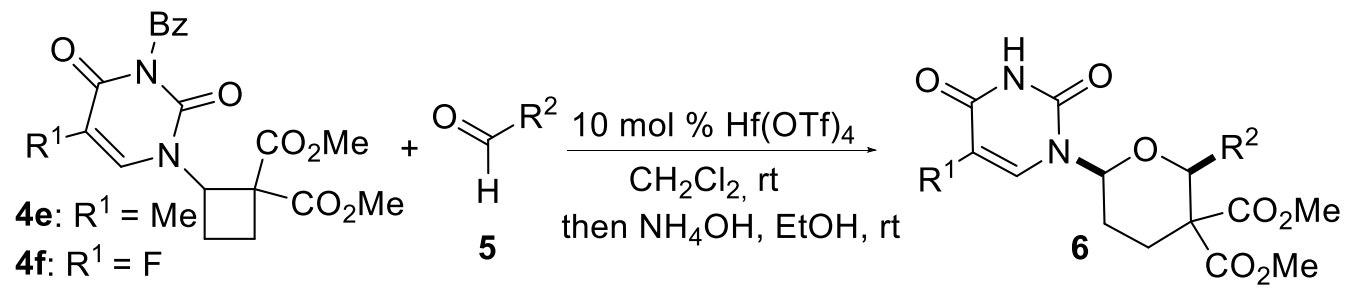

$\mathrm{Hf}(\mathrm{OTf})_{4}(21 \mathrm{mg}, 0.030 \mathrm{mmol}, 0.1 \mathrm{eq})$ was added in a flame dried $25 \mathrm{~mL}$ flask and under nitrogen atmosphere. Then, dry dichloromethane $(4.5 \mathrm{~mL})$ and aldehyde $5(0.45 \mathrm{mmol}, 1.5 \mathrm{eq})$ were added into the flask. A solution of cyclobutane $4(0.30 \mathrm{mmol}, 1.0 \mathrm{eq})$ in dry dichloromethane $(1.5 \mathrm{~mL})$ was added dropwise to the reaction mixture and stirred at room temperature for 15 minutes. The reaction mixture was filtrated on a pad of silica eluting with a mixture of ethyl acetate/ pentane (8:2) and concentrated under reduce pressure. The crude product was dissolved in ethanol $(6 \mathrm{~mL})$ and ammonium hydroxide solution $(1.8 \mathrm{~mL}, 60 \mathrm{eq}, 25 \%)$ was added. The resulting solution was stirred for 16 hours. The solvent was removed under reduced pressure and the crude product was dissolved in ethyl acetate $(25 \mathrm{~mL})$ and extracted with water $(3 \times 50 \mathrm{~mL})$. The organic layer was dried over magnesium sulfate, filtrated and concentrated under reduced pressure. The crude mixture was purified by column chromatography eluting with a mixture of ethyl acetate/ pentane (5:5 to 9:1). The product was recrystallized in ethanol (2 mL).

\section{General Procedure for the [4+2] annulation with enol ethers (GP6)}

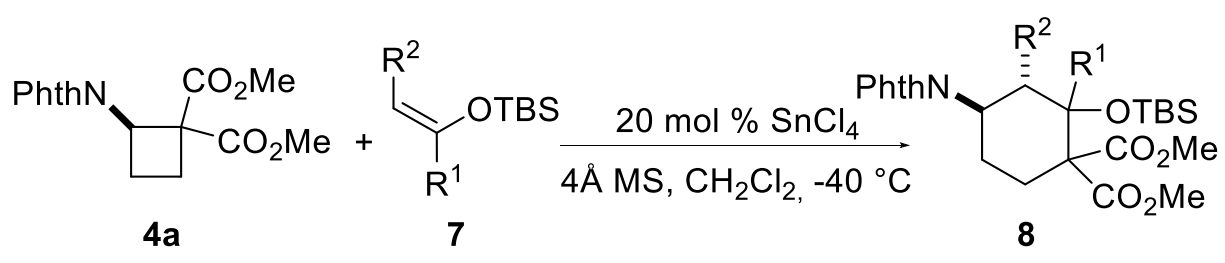

$4 \AA$ MS pellets (ca $20 \mathrm{mg}$ ) were added in an oven dried $5 \mathrm{~mL}$ round bottom flask. The flask was closed with a silicon septum and three cycles of vacuum/ $\mathrm{N}_{2}$ were performed. Aminocyclobutane $\mathbf{4 a}(0.200 \mathrm{mmol}$, $1 \mathrm{eq})$ and silyl enol ether $7(0.300 \mathrm{mmol}, 1.5 \mathrm{eq})$ were dissolved in dichloromethane $(2 \mathrm{~mL})$ and added to the reaction flask. The solution was cooled to $-40{ }^{\circ} \mathrm{C}$ using an acetonitrile/ $\mathrm{N}_{2}$ bath. A solution of tin tetrachloride $(0.43 \mathrm{~mol} / \mathrm{L}, 93.0 \mu \mathrm{L}, 0.0400 \mathrm{mmol}, 0.2 \mathrm{eq}$,) was added dropwise and the reaction was stirred at $-40{ }^{\circ} \mathrm{C}$ until full conversion was observed by NMR. The reaction was then quenched by adding triethylamine $(0.1 \mathrm{~mL})$ and the solvent was removed under reduced pressure. The reaction was purified by column chromatography on Biotage (SNAP cartridge KP-SIL 25 g, 95:5 to 4:6 Hexane/Ethyl acetate) affording the aminocyclohexanes.

\subsection{Scope of the $[4+2]$ annulation}

\section{Dimethyl 6-(1,3-dioxoisoindolin-2-yl)-2-phenyldihydro-2H-pyran-3,3(4H)-dicarboxylate (6aa)}




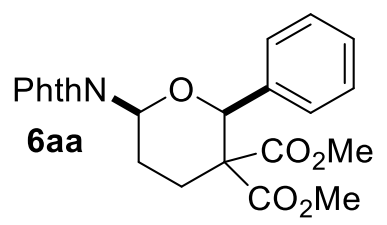

Following the general procedure GP2, dimethyl 2-(1,3-dioxoisoindolin-2-yl)cyclobutane-1,1dicarboxylate (4a) $(63.5 \mathrm{mg}, 0.200 \mathrm{mmol}, 1 \mathrm{eq})$ and benzaldehyde (5a) $(31.8 \mathrm{mg}, 0.300 \mathrm{mmol}, 1.5 \mathrm{eq})$ were stirred at room temperature for $2.5 \mathrm{~h}$. The crude oil was purified by column chromatography $\left(\mathrm{SiO}_{2}\right.$, 8:2 Pentane/Ethyl Acetate) affording dimethyl 6-(1,3-dioxoisoindolin-2-yl)-2-phenyldihydro-2H-pyran3,3(4H)-dicarboxylate (6aa) $(78.0 \mathrm{mg}, 0.184 \mathrm{mmol}, 92 \%$ yield) as a white solid.

dr 16:1, diastereoisomeric ratio was calculated in ${ }^{1} \mathrm{H}$ NMR of the isolated product integrating peaks at 5.82 and 5.67.

The major cis diastereoisomer was characterized. A strong correlation between the signals at 5.67 and 5.21 was observed. In addition, a large coupling constant $(J=11.6 \mathrm{~Hz})$ of the signal at 5.67 suggests that this proton is in axial position in anti-periplanar relationship with one proton of the neighboring $\mathrm{CH}_{2}$.

\section{$1 \mathrm{mmol}$ scale reaction :}

In a glovebox, scandium triflate $(24.6 \mathrm{mg}, 0.0500 \mathrm{mmol}, 0.05 \mathrm{eq})$ was added to a microwave vial. The vial was sealed with a Teflon septum and taken out of the glovebox. Dry dichloromethane $(4 \mathrm{~mL})$ was added. The cyclobutane $4 \mathbf{a}$ ( $317 \mathrm{mg}, 1 \mathrm{mmol}, 1 \mathrm{eq})$ was dissolved in dry dichloromethane $(3 \mathrm{~mL})$ and added. The benzaldehyde (5a) $(159 \mathrm{mg}, 1.50 \mathrm{mmol}, 1.5 \mathrm{eq})$ was then dissolved in dry dichloromethane $(3 \mathrm{~mL})$ and added. The reaction was stirred at room temperature for $5 \mathrm{~h}$, and then was filtered over a pad of alumina, eluted with ethyl acetate and concentrated under vacuum. The crude product was purified by column chromatography ( $\mathrm{SiO}_{2}, 8: 2$ Pentane/Ethyl Acetate) affording dimethyl 6-(1,3-dioxoisoindolin-2-yl)-2phenyldihydro-2H-pyran-3,3(4H)-dicarboxylate (6aa) $(342 \mathrm{mg}, 0.808 \mathrm{mmol}, 81 \%$ yield) as a white solid.

dr 13:1, diastereoisomeric ratio was calculated in ${ }^{1} \mathrm{H}$ NMR of the isolated product integrating peaks at 5.82 and 5.67 .

$\mathbf{R}_{f} 0.25$ (7:3 Pentane/Ethyl Acetate).

Mp $167-170{ }^{\circ} \mathrm{C}$.

${ }^{1} \mathbf{H}$ NMR $\left(400 \mathrm{MHz}, \mathrm{CDCl}_{3}\right)^{9} \delta 7.87(\mathrm{dd}, J=5.5,3.1 \mathrm{~Hz}, 2 \mathrm{H}, \mathrm{Phth}), 7.73(\mathrm{dd}, J=5.5,3.0 \mathrm{~Hz}, 2 \mathrm{H}, \mathrm{Phth})$, 7.40 - 7.36 (m, $2 \mathrm{H}, \mathrm{Ph}), 7.27-7.20$ (m, $3 \mathrm{H}, \mathrm{Ph}), 5.67$ (dd, J = 11.6, 3.0 Hz, $1 \mathrm{H}, \mathrm{N}-\mathrm{C}(H)-\mathrm{O}), 5.21$ (s, 1 $\mathrm{H}, \mathrm{O}-\mathrm{C}(H)-\mathrm{Ph}), 3.68\left(\mathrm{~s}, 3 \mathrm{H}, \mathrm{OCH}_{3}\right), 3.63\left(\mathrm{~s}, 3 \mathrm{H}, \mathrm{OCH}_{3}\right), 3.35-3.21\left(\mathrm{~m}, 1 \mathrm{H}, \mathrm{CH}_{2}\right), 2.79$ (ddd, $J=13.7$, 4.4, 3.1 Hz, $1 \mathrm{H}, \mathrm{CH}_{2}$ ), 2.29 (ddd, $J=13.6,13.6,4.3 \mathrm{~Hz}, 1 \mathrm{H}, \mathrm{CH}_{2}$ ), 1.78 (dddd, $J=13.5,4.3,3.0,3.0 \mathrm{~Hz}$, $1 \mathrm{H}, \mathrm{CH}_{2}$ ).

${ }^{13}$ C NMR $\left(101 \mathrm{MHz}, \mathrm{CDCl}_{3}\right) \delta 170.9,168.6,167.0,138.3,134.3,131.8,127.8,127.4,127.3,123.6,82.2$, 79.3, 58.1, 52.6, 51.9, 32.3, 23.8 .

IR 2953 (w), 1781 (w), 1722 (s), 1450 (w), 1440 (w), 1375 (m), 1262 (m), 1209 (w), 1082 (m), 1050 (m), $1012(\mathrm{w})$.

HRMS (ESI) calcd for $\mathrm{C}_{23} \mathrm{H}_{21} \mathrm{NNaO}_{7}^{+}[\mathrm{M}+\mathrm{Na}]^{+} 446.1216$; found 446.1213.

\footnotetext{
${ }^{9}$ Small amount of other compound (less than 5\%) observed by ${ }^{1} \mathrm{H}$ NMR (probably other diastereoisomer).
} 


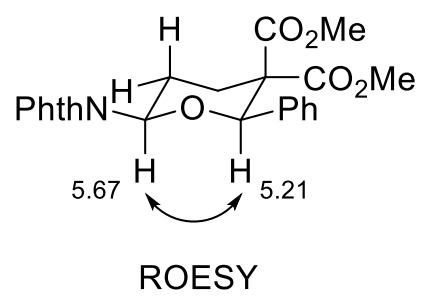

Dimethyl 2-(4-chlorophenyl)-6-(1,3-dioxoisoindolin-2-yl)dihydro-2H-pyran-3,3(4H)-dicarboxylate (6ab)

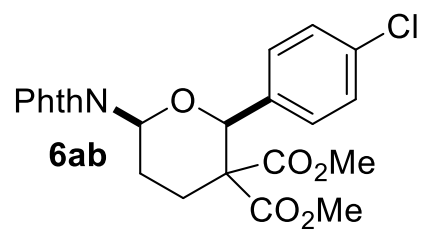

Following the general procedure GP2, dimethyl 2-(1,3-dioxoisoindolin-2-yl)cyclobutane-1,1dicarboxylate (4a) $(63.5 \mathrm{mg}, 0.200 \mathrm{mmol}, 1 \mathrm{eq})$ and 4-chlorobenzaldehyde (5b) $(42.2 \mathrm{mg}, 0.300 \mathrm{mmol}$, $1.5 \mathrm{eq})$ were stirred at room temperature for $2.5 \mathrm{~h}$. The crude oil was purified by column chromatography $\left(\mathrm{SiO}_{2}, 8: 2\right.$ Pentane/Ethyl Acetate) affording dimethyl 2-(4-chlorophenyl)-6-(1,3-dioxoisoindolin-2yl)dihydro-2H-pyran-3,3(4H)-dicarboxylate (6ab) $(69.0 \mathrm{mg}, 0.151 \mathrm{mmol}, 75 \%$ yield) as a white solid.

dr 17:1, diastereoisomeric ratio was calculated in ${ }^{1} \mathrm{H}$ NMR of the isolated product integrating peaks at 5.86 and 5.6.

The major diastereoisomer is the cis, in analogy with the ${ }^{1} \mathrm{H}$ NMR spectrum of $6 \mathbf{a a}$.

$\mathbf{R}_{f} 0.33$ (7:3 Pentane/Ethyl Acetate).

Mp $169-176^{\circ} \mathrm{C}$.

${ }^{1} \mathbf{H}$ NMR $\left(400 \mathrm{MHz}, \mathrm{CDCl}_{3}\right)^{9}$ only maj diastereoisomer characterized $\delta 7.87(\mathrm{dd}, J=5.5,3.0 \mathrm{~Hz}, 2 \mathrm{H}$, Phth), 7.74 (dd, $J=5.5,3.0 \mathrm{~Hz}, 2 \mathrm{H}, \mathrm{Phth}), 7.34-7.28$ (m, $2 \mathrm{H}, \mathrm{Ph}), 7.23-7.18$ (m, $2 \mathrm{H}, \mathrm{Ph}), 5.66$ (dd, $J$ $=11.7,3.0 \mathrm{~Hz}, 1 \mathrm{H}, \mathrm{N}-\mathrm{C}(H)-\mathrm{O}), 5.16(\mathrm{~s}, 1 \mathrm{H}, \mathrm{O}-\mathrm{C}(H)-\mathrm{Ph}), 3.68\left(\mathrm{~s}, 3 \mathrm{H}, \mathrm{OCH}_{3}\right), 3.65\left(\mathrm{~s}, 3 \mathrm{H}, \mathrm{OCH}_{3}\right), 3.27$ (dddd, $J=13.5,13.5,11.6,4.4 \mathrm{~Hz}, 1 \mathrm{H}), 2.78$ (ddd, $J=13.7,4.3,2.9 \mathrm{~Hz}, 1 \mathrm{H}$ ), 2.27 (ddd, $J=13.6,13.6$, $4.3 \mathrm{~Hz}, 1 \mathrm{H}$ ), 1.78 (dddd, $J=13.5,4.3,3.0,3.0 \mathrm{~Hz}, 1 \mathrm{H}$ ).

${ }^{13} \mathbf{C}$ NMR $\left(101 \mathrm{MHz}, \mathrm{CDCl}_{3}\right)$ only maj diastereoisomer characterized $\delta 170.8,168.3,167.0,136.8,134.4$, $133.5,131.7,128.9,127.4,123.7,81.6,79.3,58.0,52.7,52.0,32.2,23.7$.

IR 2953 (w), 1782 (w), 1724 (s), 1439 (w), 1375 (s), 1344 (w), 1262 (m), 1210 (m), 1075 (m), 1052 (m), $1014(\mathrm{w})$.

HRMS (ESI) calcd for $\mathrm{C}_{23} \mathrm{H}_{20} \mathrm{ClNNaO}_{7}^{+}[\mathrm{M}+\mathrm{Na}]^{+} 480.0820$; found 480.0820 .

Dimethyl 6-(1,3-dioxoisoindolin-2-yl)-2-(4-methoxyphenyl)dihydro-2H-pyran-3,3(4H)-dicarboxylate (6ac)

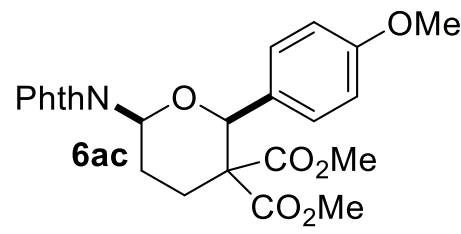

Following the general procedure GP2, dimethyl 2-(1,3-dioxoisoindolin-2-yl)cyclobutane-1,1dicarboxylate (4a) $(63.5 \mathrm{mg}, 0.200 \mathrm{mmol}, 1 \mathrm{eq})$ and 4-methoxybenzaldehyde (5c) $(40.8 \mathrm{mg}, 0.300 \mathrm{mmol}$, $1.5 \mathrm{eq})$ were stirred at room temperature for $2.5 \mathrm{~h}$. The crude oil was purified by column chromatography 
$\left(\mathrm{SiO}_{2}, \quad 8: 2\right.$ to 6:4 Pentane/Ethyl Acetate) affording dimethyl 6-(1,3-dioxoisoindolin-2-yl)-2-(4methoxyphenyl)dihydro-2H-pyran-3,3(4H)-dicarboxylate (6ac) $(74.6 \mathrm{mg}, 0.165 \mathrm{mmol}, 82 \%$ yield) as a white solid.

dr 6:1, diastereoisomeric ratio was calculated in ${ }^{1} \mathrm{H}$ NMR of the isolated product integrating peaks at 5.79 and 5.65 .

The major diastereoisomer is the cis, in analogy with the ${ }^{1} \mathrm{H}$ NMR spectrum of $6 \mathbf{a a}$.

$\mathbf{R}_{f} 0.22$ (7:3 Pentane/Ethyl Acetate).

Mp $154-161^{\circ} \mathrm{C}$.

${ }^{1} \mathbf{H}$ NMR $\left(400 \mathrm{MHz}, \mathrm{CDCl}_{3}\right)^{9}$ on a $6($ maj $): 1(\mathrm{~min})$ mixture $\delta 7.89-7.77(\mathrm{~m}, 2.3 \mathrm{H}$, Phth $\mathrm{maj}+$ min $), 7.75-$ 7.65 (m, 2.3 H, Phth maj + min), 7.37 - 7.33 (m, $0.3 \mathrm{H}, \mathrm{Ph}$ min), 7.33 - 7.27 (m, $2 \mathrm{H}, \mathrm{Ph}$ maj), 6.86 - 6.80 (m, $0.3 \mathrm{H}, \mathrm{Ph}$ min), $6.80-6.73$ (m, $2 \mathrm{H}, \mathrm{Ph}$ min), 6.08 (s, $0.15 \mathrm{H}, \mathrm{O}-\mathrm{C}(H)-\mathrm{Ph}$ min), 5.79 (dd, $J=10.4,5.3$ $\mathrm{Hz}, 0.15 \mathrm{H}, \mathrm{N}-\mathrm{C}(H)-\mathrm{O}$ min), 5.65 (dd, $J=11.7,3.0 \mathrm{~Hz}, 1 \mathrm{H}, \mathrm{N}-\mathrm{C}(H)-\mathrm{O}$ maj), 5.13 (s, $1 \mathrm{H}, \mathrm{O}-\mathrm{C}(H)-\mathrm{Ph}$ maj), 3.87 (s, $0.45 \mathrm{H}, \mathrm{OCH}_{3}$ min), 3.76 (s, $0.45 \mathrm{H}, \mathrm{OCH}_{3}$ min), 3.74 (s, $3 \mathrm{H}, \mathrm{OCH}_{3}$ maj), 3.67(s, $3 \mathrm{H}$, $\mathrm{OCH}_{3}$ maj), $3.66\left(\mathrm{~s}, 3 \mathrm{H}, \mathrm{OCH}_{3}\right.$ maj), $3.40\left(\mathrm{~s}, 0.45 \mathrm{H}, \mathrm{OCH}_{3} \mathrm{~min}\right), 3.28\left(\mathrm{~m}, 1 \mathrm{H}, \mathrm{CH}_{2}\right.$ maj), $2.85-2.69$ (m, $1.15 \mathrm{H}, \mathrm{CH}_{2}$ maj + min), $2.69-2.58\left(\mathrm{~m}, 0.3 \mathrm{H}, \mathrm{CH}_{2} \min \right), 2.26\left(\mathrm{ddd}, J=13.6,13.6,4.3 \mathrm{~Hz}, 1 \mathrm{H}, \mathrm{CH}_{2}\right.$ maj), $1.97-1.88\left(\mathrm{~m}, 0.15 \mathrm{H}, \mathrm{CH}_{2}\right.$ min), $1.81-1.72\left(\mathrm{~m}, 1 \mathrm{H}, \mathrm{CH}_{2}\right.$ maj). ${ }^{10}$

${ }^{13} \mathbf{C}$ NMR $\left(101 \mathrm{MHz}, \mathrm{CDCl}_{3}\right)$ only maj diastereoisomer characterized $\delta 170.9,168.6,167.0,159.1,134.3$, $131.8,130.4,128.7,123.6,112.6,82.0,79.3,58.1,55.1,52.6,52.0,32.2,23.8$.

IR 2954 (w), 1782 (w), 1725 (s), 1615 (w), 1515 (w), 1462 (w), 1440 (w), 1376 (m), 1347 (w), 1253 (m), $1210(\mathrm{w}), 1076(\mathrm{~m}), 1045(\mathrm{~m})$.

HRMS (ESI) calcd for $\mathrm{C}_{24} \mathrm{H}_{23} \mathrm{NNaO}_{8}{ }^{+}[\mathrm{M}+\mathrm{Na}]^{+}$476.1316; found 476.1319.

Dimethyl 6-(1,3-dioxoisoindolin-2-yl)-2-(2-methoxyphenyl)dihydro-2H-pyran-3,3(4H)-dicarboxylate (6ad)

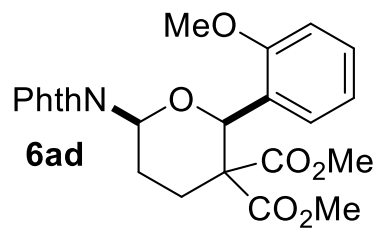

Following the general procedure GP2, dimethyl 2-(1,3-dioxoisoindolin-2-yl)cyclobutane-1,1dicarboxylate (4a) $(63.5 \mathrm{mg}, 0.200 \mathrm{mmol}, 1 \mathrm{eq})$ and 2-methoxybenzaldehyde (5d) (40.8 mg, $0.300 \mathrm{mmol}$, $1.5 \mathrm{eq})$ were stirred at room temperature for $2.5 \mathrm{~h}$. The crude oil was purified by column chromatography $\left(\mathrm{SiO}_{2}, \quad 65: 35\right.$ Pentane/Ethyl Acetate) affording dimethyl 6-(1,3-dioxoisoindolin-2-yl)-2-(2methoxyphenyl)dihydro-2H-pyran-3,3(4H)-dicarboxylate (6ad) $(82.8 \mathrm{mg}, 0.183 \mathrm{mmol}, 91 \%$ yield) as a white solid.

dr 8.5:1, diastereoisomeric ratio was calculated in ${ }^{1} \mathrm{H}$ NMR of the isolated product integrating peaks at 6.37 and 5.25 .

The major diastereoisomer is the cis, in analogy with the ${ }^{1} \mathrm{H}$ NMR spectrum of $6 \mathbf{a a}$.

$\mathbf{R}_{f} 0.18$ (7:3 Pentane/Ethyl Acetate).

Mp $87-97^{\circ} \mathrm{C}$.

${ }^{1} \mathbf{H}$ NMR $\left(400 \mathrm{MHz}, \mathrm{CDCl}_{3}\right)^{9}$ only maj diastereoisomer characterized $\delta 7.90-7.81$ (m, $\left.2 \mathrm{H}, \mathrm{Phth}\right), 7.81-$ 7.66 (m, 2 H, Phth, 1 H, Ph), $7.24-7.16$ (m, 1 H, Ph), 6.93 (dd, J = 7.5 Hz, 1 H, Ph), 6.75 (d, J = 8.1 Hz,

${ }^{10}$ Over-integrated due to overlap with water in $\mathrm{CDCl}_{3}$. 
$1 \mathrm{H}, \mathrm{Ph}), 5.64(\mathrm{dd}, J=11.5,3.0 \mathrm{~Hz}, 1 \mathrm{H}, \mathrm{N}-\mathrm{C}(H)-\mathrm{O}), 5.25$ (s, $1 \mathrm{H}, \mathrm{O}-\mathrm{C}(H)-\mathrm{Ph}), 3.76\left(\mathrm{~s}, 3 \mathrm{H}, \mathrm{OCH}_{3}\right), 3.75$ $\left(\mathrm{s}, 3 \mathrm{H}, \mathrm{OCH}_{3}\right), 3.55\left(\mathrm{~s}, 3 \mathrm{H}, \mathrm{OCH}_{3}\right), 3.03-2.85\left(\mathrm{~m}, 1 \mathrm{H}, \mathrm{CH}_{2}\right), 2.72$ (ddd, $J=14.1,3.8,3.8 \mathrm{~Hz}, 1 \mathrm{H}$, $\mathrm{CH}_{2}$ ), $2.61-2.50\left(\mathrm{~m}, 1 \mathrm{H}, \mathrm{CH}_{2}\right), 1.80$ (dddd, $J=13.5,3.5,3.5,3.5 \mathrm{~Hz}, 1 \mathrm{H}, \mathrm{CH}_{2}$ ).

${ }^{13} \mathbf{C}$ NMR $\left(101 \mathrm{MHz}, \mathrm{CDCl}_{3}\right)$ only maj diastereoisomer characterized $\delta 169.4,168.9,167.1,155.8,134.3$, $131.8,130.5,128.8,126.3,123.6,119.9,108.8,79.4,76.4,57.6,55.2,52.3,52.1,32.1,24.2$.

IR 2954 (w), 1765 (w), 1724 (s), 1600 (w), 1495 (w), 1464 (w), 1439 (w), 1376 (m), 1293 (w), 1256 (m), $1200(\mathrm{w}), 1111(\mathrm{w}), 1073(\mathrm{~m}), 1050(\mathrm{~m})$.

HRMS (ESI) calcd for $\mathrm{C}_{24} \mathrm{H}_{23} \mathrm{NNaO}_{8}{ }^{+}[\mathrm{M}+\mathrm{Na}]^{+}$476.1316; found 476.1312.

(E)-Dimethyl 6-(1,3-dioxoisoindolin-2-yl)-2-styryldihydro-2H-pyran-3,3(4H)-dicarboxylate (6ae)

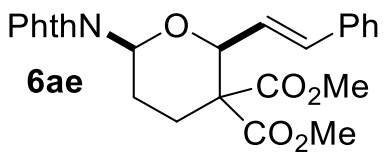

Following the general procedure GP2, dimethyl 2-(1,3-dioxoisoindolin-2-yl)cyclobutane-1,1dicarboxylate (4a) $(63.5 \mathrm{mg}, 0.200 \mathrm{mmol}, 1 \mathrm{eq})$ and cinnamaldehyde (5e) $(39.6 \mathrm{mg}, 0.300 \mathrm{mmol}, 1.5 \mathrm{eq})$ were stirred at room temperature for $2.5 \mathrm{~h}$. The crude oil was purified by column chromatography $\left(\mathrm{SiO}_{2}\right.$, 9:1 to 8:2 Pentane/Ethyl Acetate) affording (E)-dimethyl 6-(1,3-dioxoisoindolin-2-yl)-2-styryldihydro-2Hpyran-3,3(4H)-dicarboxylate (6ae) $(85.7 \mathrm{mg}, 0.191 \mathrm{mmol}, 95 \%$ yield) as a white solid.

dr 2:1, diastereoisomeric ratio was calculated in ${ }^{1} \mathrm{H}$ NMR of the isolated product integrating peaks at 5.84 and 5.63 .

The major diastereoisomer is the cis, in analogy with the ${ }^{1} \mathrm{H}$ NMR spectrum of $6 \mathbf{a a}$.

$\mathbf{R}_{f} 0.3$ (7:3 Pentane/Ethyl Acetate).

Mp 88-92 ${ }^{\circ} \mathrm{C}$.

${ }^{1} \mathbf{H}$ NMR $\left(400 \mathrm{MHz}, \mathrm{CDCl}_{3}\right)^{9}$ on a $2(\mathrm{maj}): 1(\mathrm{~min})$ mixture $\delta 7.90-7.81(\mathrm{~m}, 3 \mathrm{H}$, Phth maj + min), $7.79-$ 7-68 (m, $3 \mathrm{H}$, Phth maj + min), $7.45-7.39$ (m, $1 \mathrm{H}, \mathrm{Ph}$ min), $7.38-7.31$ (m, $3 \mathrm{H}, \mathrm{Ph}$ maj + min), $7.30-$ $7.17\left(\mathrm{~m}, 3.5 \mathrm{H}, \mathrm{Ph}\right.$ maj + min) ${ }^{11} 6.85(\mathrm{dd}, J=15.8,1.5 \mathrm{~Hz}, 0.5 \mathrm{H}, \mathrm{CH}$ vinyl $\min$ ), 6.67 (dd, $J=16.0,7.2$ $\mathrm{Hz}, 1 \mathrm{H}, \mathrm{CH}$ vinyl maj), $6.54(\mathrm{~d}, J=16.1 \mathrm{~Hz}, 1 \mathrm{H}, \mathrm{CH}$ vinyl maj), $6.28(\mathrm{dd}, J=15.8,6.4 \mathrm{~Hz}, 0.5 \mathrm{H}, \mathrm{CH}$ vinyl min), $5.84(\mathrm{dd}, J=11.2,3.4 \mathrm{~Hz}, 0.5 \mathrm{H}, \mathrm{N}-\mathrm{C}(H)-\mathrm{O}$ min), $5.63(\mathrm{dd}, J=11.8,3.0 \mathrm{~Hz}, 1 \mathrm{H}, \mathrm{N}-\mathrm{C}(H)-\mathrm{O}$ maj), 5.47 (ddd, $J=6.4,1.5,1.5 \mathrm{~Hz}, 0.5 \mathrm{H}, \mathrm{O}-\mathrm{C}(H)$-vinyl $\min$ ), 4.60 (dd, $J=7.2,0.9 \mathrm{~Hz}, 1 \mathrm{H}, \mathrm{O}-\mathrm{C}(H)$ vinyl maj), $3.94\left(\mathrm{~s}, 1.5 \mathrm{H}, \mathrm{OCH}_{3} \min \right), 3.90\left(\mathrm{~s}, 3 \mathrm{H}, \mathrm{OCH}_{3}\right.$ maj), $3.70\left(\mathrm{~s}, 1.5 \mathrm{H}, \mathrm{OCH}_{3} \min \right), 3.67(\mathrm{~s}, 3 \mathrm{H}$, $\mathrm{OCH}_{3}$ maj), 3.08 (dddd, $J=13.7,13.7,11.7,4.4 \mathrm{~Hz}, 1 \mathrm{H}, \mathrm{CH}_{2}$ maj), $2.88-2.78$ (m, $0.5 \mathrm{H}, \mathrm{CH}_{2}$ min), 2.75 (ddd, $J=13.6,4.4,2.8 \mathrm{~Hz}, 1 \mathrm{H}, \mathrm{CH}_{2}$ maj), 2.67 (dddd, $J=14.3,3.9,3.9,1.6 \mathrm{~Hz}, 0.5 \mathrm{H}, \mathrm{CH}_{2} \min$ ), 2.37 (ddd, $J=13.9,13.9,4.1 \mathrm{~Hz}, 0.5 \mathrm{H}, \mathrm{CH}_{2} \min$ ), 2.23 (ddd, $J=13.7,13.7,4.2 \mathrm{~Hz}, 1 \mathrm{H}, \mathrm{CH}_{2}$ maj), 1.82 (dddd, $J=13.8,3.9,3.9,3.9 \mathrm{~Hz}, 0.5 \mathrm{H}, \mathrm{CH}_{2}$ min), 1.75 (dddd, $J=13.6,13.6,4.2,2.8 \mathrm{~Hz}, 1 \mathrm{H}, \mathrm{CH}_{2}$ maj).

${ }^{13}$ C NMR $\left(101 \mathrm{MHz}, \mathrm{CDCl}_{3}\right) \delta 170.4,169.5,168.9,168.8,167.4,167.1,136.5,136.2,135.9,134.4$, 134.3, 132.9, 131.8, 131.7, 128.6, 128.4, 128.2, 127.8, 126.8, 126.8, 125.9, 123.6, 123.6, 121.7, 82.8, 79.0, $77.2,72.9,58.1,57.1,53.3,53.0,52.8,52.6,31.6,25.2,23.8,23.7$.

IR 3027 (w), 2953 (w), 1781 (w), 1724 (s), 1452 (w), 1442 (w), 1376 (m), 1269 (w), 1253 (w), 1209 (w), $1071(\mathrm{~m}), 1010(\mathrm{w})$.

HRMS (ESI) calcd for $\mathrm{C}_{25} \mathrm{H}_{23} \mathrm{NNaO}_{7}^{+}[\mathrm{M}+\mathrm{Na}]^{+}$472.1367; found 472.1371.

\section{Dimethyl 6-(1,3-dioxoisoindolin-2-yl)-5-methyl-2-phenyldihydro-2H-pyran-3,3(4H)-dicarboxylate (6ba)}

${ }^{11}$ Over-integrated due to overlap with $\mathrm{CHCl}_{3}$ peak. 


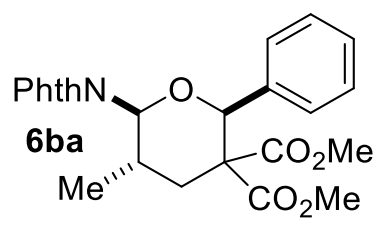

Following the general procedure GP3, dimethyl 2-(1,3-dioxoisoindolin-2-yl)-3-methylcyclobutane1,1-dicarboxylate (4b) $(66.3 \mathrm{mg}, 0.200 \mathrm{mmol}, 1 \mathrm{eq})$ and benzaldehyde (5a) $(31.8 \mathrm{mg}, 0.300 \mathrm{mmol}, 1.5 \mathrm{eq})$ were stirred at room temperature for $5 \mathrm{~h}$. The crude oil was purified by column chromatography $\left(\mathrm{SiO}_{2}, 8: 2\right.$ Pentane/Ethyl Acetate) affording dimethyl 6-(1,3-dioxoisoindolin-2-yl)-5-methyl-2-phenyldihydro-2Hpyran-3,3(4H)-dicarboxylate (6ba) $(56.0 \mathrm{mg}, 0.128 \mathrm{mmol}, 64 \%$ yield) as a white solid.

dr 5:1, diastereoisomeric ratio was calculated in ${ }^{1} \mathrm{H}$ NMR of the isolated product integrating peaks at 2.73 and 2.56 .

The major diastereoisomer is the cis-trans, in analogy with the ${ }^{1} \mathrm{H}$ NMR spectrum of $\mathbf{6 b b}$.

$\mathbf{R}_{f} 0.36$ ( $7: 3$ Pentane/Ethyl Acetate).

Mp 79-86 ${ }^{\circ} \mathrm{C}$.

${ }^{1} \mathbf{H}$ NMR $\left(400 \mathrm{MHz}, \mathrm{CDCl}_{3}\right)$ on a $5(\mathrm{maj}): 1(\mathrm{~min})$ mixture $\delta 7.88(\mathrm{dd}, J=5.4,3.0 \mathrm{~Hz}, 2 \mathrm{H}$, Phth maj), 7.82 (dd, $J=5.5,3.1 \mathrm{~Hz}, 0.4 \mathrm{H}$, Phth $\mathrm{min}$ ), 7.74 (dd, $J=5.5,3.0 \mathrm{~Hz}, 2 \mathrm{H}$, Phth maj), 7.71 (dd, $J=5.5,3.1 \mathrm{~Hz}$, $0.4 \mathrm{H}$, Phth min) 7.45 - 7.41 (m, 0.4 H, Ph min), 7.40 - 7.35 (m, 2 H, Ph maj), $7.34-7.18$ (m, 3.6 H, Ph maj + min $){ }^{11} 6.24(\mathrm{~s}, 0.2 \mathrm{H}, \mathrm{O}-\mathrm{C}(H)-\mathrm{Ph} \min ), 5.33(\mathrm{~d}, J=10.5 \mathrm{~Hz}, 0.2 \mathrm{H}, \mathrm{N}-\mathrm{C}(H)-\mathrm{O} \min ), 5.26(\mathrm{~d}, J=$ $10.6 \mathrm{~Hz}, 1 \mathrm{H}, \mathrm{N}-\mathrm{C}(H)-\mathrm{O}$ maj), 5.18 (s, $1 \mathrm{H}, \mathrm{O}-\mathrm{C}(H)-\mathrm{Ph}$ maj), 3.89 (s, $0.6 \mathrm{H}, \mathrm{OCH}_{3}$ min), 3.68 (s, $3 \mathrm{H}$, $\mathrm{OCH}_{3}$ maj), 3.62 (s, $3 \mathrm{H}, \mathrm{OCH}_{3}$ maj), 3.47 - 3.34 (m, $1 \mathrm{H}, \mathrm{Me}-\mathrm{CH}$ maj), 3.33 (s, $0.6 \mathrm{H}, \mathrm{OCH}_{3}$ min), 3.09 $2.96(\mathrm{~m}, 0.2 \mathrm{H}, \mathrm{Me}-\mathrm{CH} \min ), 2.72\left(\mathrm{dd}, J=13.7,4.2 \mathrm{~Hz}, 1 \mathrm{H}, \mathrm{CH}_{2}\right.$ maj), 2.55 (dd, $J=14.2,3.6 \mathrm{~Hz}, 0.2 \mathrm{H}$, $\mathrm{CH}_{2}$ min), 2.42 (dd, $J=14.2,13.1 \mathrm{~Hz}, 0.2 \mathrm{H}, \mathrm{CH}_{2}$ min), 1.95 (dd, $J=13.7,12.4 \mathrm{~Hz}, 1 \mathrm{H}, \mathrm{CH}_{2}$ maj), 0.95 (d, $J=8.0 \mathrm{~Hz}, 0.6 \mathrm{H}, \mathrm{CH}_{3}$ min), 0.93 (d, $J=6.8 \mathrm{~Hz}, 3 \mathrm{H}, \mathrm{CH}_{3}$ maj).

${ }^{13}$ C NMR $\left(101 \mathrm{MHz}, \mathrm{CDCl}_{3}\right)^{12} \delta 170.7,169.5,168.7,167.9,167.1,138.2,137.5,134.34,134.30,131.7$, 128.1, 128.0, 127.8, 127.6, 127.4, 127.3, 123.6, 123.5, 84.9, 82.2, 81.0, 77.3, 76.2, 59.9, 59.3, 53.2, 52.6, $52.5,51.9,40.5,34.4,27.7,27.0,16.9,16.2$.

IR 2956 (w), 1774 (w), 1727 (s), 1461 (w), 1439 (w), 1376 (m), 1261 (m), 1214 (w), 1090 (w), 1026 (w).

HRMS (ESI) calcd for $\mathrm{C}_{24} \mathrm{H}_{23} \mathrm{NNaO}_{7}^{+}[\mathrm{M}+\mathrm{Na}]^{+} 460.1367$; found 460.1367 .

\section{Dimethyl 2-(4-chlorophenyl)-6-(1,3-dioxoisoindolin-2-yl)-5-methyldihydro-2H-pyran-3,3(4H)- dicarboxylate (6bb)}

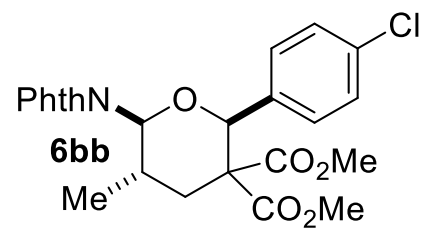

Following the general procedure GP3, dimethyl 2-(1,3-dioxoisoindolin-2-yl)-3-methylcyclobutane1,1-dicarboxylate (4b) $(66.3 \mathrm{mg}, 0.200 \mathrm{mmol}, 1 \mathrm{eq})$ and 4-chlorobenzaldehyde (5b) (42.2 $\mathrm{mg}, 0.300$ mmol, $1.5 \mathrm{eq})$ were stirred at room temperature for $5 \mathrm{~h}$. The crude oil was purified by column chromatography $\left(\mathrm{SiO}_{2}, 8: 2\right.$ Pentane/Ethyl Acetate) affording dimethyl 2-(4-chlorophenyl)-6-(1,3dioxoisoindolin-2-yl)-5-methyldihydro-2H-pyran-3,3(4H)-dicarboxylate (6bb) (58.0 mg, $0.123 \mathrm{mmol}$, $62 \%$ yield) as a white solid.

dr 5:1, diastereoisomeric ratio was calculated in ${ }^{1} \mathrm{H}$ NMR of the isolated product integrating peaks at 5.35 and 5.25 . 
The major cis-trans diastereoisomer was characterized. Strong correlations between the signals at 5.25 and 5.14, and between 5.25 and 0.92 were observed. In addition, a large coupling constant $(J=10.6 \mathrm{~Hz})$ of the signal at 5.25 suggests that this proton is in axial position in anti-periplanar relationship with the proton of the neighboring $\mathrm{CH}$.

$\mathbf{R}_{f} 0.38$ ( $7: 3$ Pentane/Ethyl Acetate).

Mp 81-90 ${ }^{\circ} \mathrm{C}$.

${ }^{1} \mathbf{H}$ NMR $\left(400 \mathrm{MHz}, \mathrm{CDCl}_{3}\right)^{9}$ on a $5(\mathrm{maj}): 1(\mathrm{~min})$ mixture $\delta 7.87(\mathrm{dd}, J=5.6,3.2 \mathrm{~Hz}, 2 \mathrm{H}$, Phth maj), 7.83 (dd, $J=5.5,3.1 \mathrm{~Hz}, 0.4 \mathrm{H}$, Phth $\mathrm{min}$ ), 7.75 (dd, $J=5.5,3.1 \mathrm{~Hz}, 2 \mathrm{H}$, Phth maj) $_{\text {) }} 7.71$ (dd, $J=5.6,2.8 \mathrm{~Hz}$, 0.4 H, Phth min), $7.41-7.35$ (m, $0.4 \mathrm{H}, \mathrm{Ph}$ min), $7.34-7.24$ (m, 2.4 H, Ph maj + min), $7.23-7.16$ (m, 2 H, Ph maj), 6.22 (s, 0.2 H, O-C (H)-Ph min), 5.35 (d, J=10.4 Hz, 0.2 H, N-C(H)-O min), 5.25 (d, J= 10.6 $\mathrm{Hz}, 1 \mathrm{H}, \mathrm{N}-\mathrm{C}(H)-\mathrm{O}$ maj), 5.14 (s, $1 \mathrm{H}, \mathrm{O}-\mathrm{C}(H)-\mathrm{Ph} m a j), 3.87$ (s, $\left.0.6 \mathrm{H}, \mathrm{OCH}_{3} \min \right), 3.68\left(\mathrm{~s}, 3 \mathrm{H}, \mathrm{OCH}_{3}\right.$ maj), 3.64 (s, $3 \mathrm{H}, \mathrm{OCH}_{3}$ maj), $3.44-3.35$ (m, $1 \mathrm{H}, \mathrm{Me}-\mathrm{CH}$ maj), 3.34 (s, $0.6 \mathrm{H}, \mathrm{OCH}_{3}$ min), $3.05-2.94$ (m, $0.2 \mathrm{H}, \mathrm{Me}-\mathrm{CH}$ min), 2.71 (dd, $J=13.7,4.2 \mathrm{~Hz}, 1 \mathrm{H}, \mathrm{CH}_{2}$ maj), 2.50 (dd, $J=14.2,3.5 \mathrm{~Hz}, 0.2 \mathrm{H}, \mathrm{CH}_{2}$ min), 2.40 (dd, $J=14.3,13.1 \mathrm{~Hz}, 0.2 \mathrm{H}, \mathrm{CH}_{2} \min$ ), 1.93 (dd, $J=13.7,12.4 \mathrm{~Hz}, 1 \mathrm{H}, \mathrm{CH}_{2}$ min), 0.95 (d, $J=$ $\left.6.6 \mathrm{~Hz}, 0.6 \mathrm{H}, \mathrm{CH}_{3} \min \right), 0.92$ (d, $J=6.6 \mathrm{~Hz}, 3 \mathrm{H}, \mathrm{CH}_{3}$ maj).

${ }^{13}$ C NMR $\left(101 \mathrm{MHz}, \mathrm{CDCl}_{3}\right)^{12} \delta 170.7,170.5,169.4,168.4,167.9,167.1,136.7,136.2,134.4,133.8$, $133.5,131.7,129.0,128.9,128.2,127.4,123.7,123.6,84.8,81.5,81.3,75.4,60.2,59.2,53.2,52.7,52.5$, $52.0,40.4,34.5,27.6,26.7,17.0,16.2$.

IR 2975 (w), 2882, (w), 1780 (w), 1726 (s), 1377 (m), 1263 (m), 1215 (w), 1084 (m), 1021 (w).

HRMS (ESI) calcd for $\mathrm{C}_{24} \mathrm{H}_{22} \mathrm{ClKNO}_{7}{ }^{+}[\mathrm{M}+\mathrm{K}]^{+}$510.0716; found 510.0715.

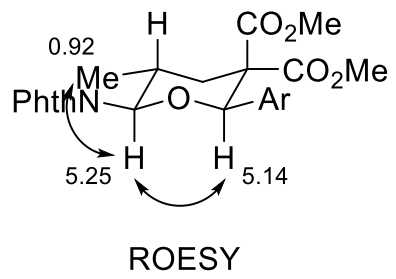

\section{Dimethyl 6-(1,3-dioxoisoindolin-2-yl)-2,5-diphenyldihydro-2H-pyran-3,3(4H)-dicarboxylate (6ca)}

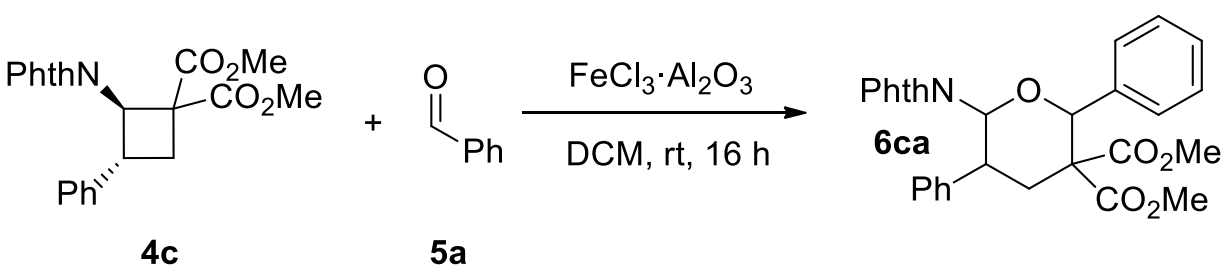

In a glovebox, iron trichloride supported on alumina $(1.00 \mathrm{mmol} / \mathrm{g}, 200 \mathrm{mg}, 0.200 \mathrm{mmol}, 1 \mathrm{eq})$ was added to a microwave vial. The vial was sealed with a Teflon septum and taken out of the glovebox. Dry dichloromethane $(2 \mathrm{~mL})$ was added. Dimethyl 2-(1,3-dioxoisoindolin-2-yl)-3-phenylcyclobutane-1,1dicarboxylate (4c) $(79.0 \mathrm{mg}, 0.200 \mathrm{mmol}, 1 \mathrm{eq})$ was dissolved in dry dichloromethane $(2 \mathrm{~mL})$ and added. Benzaldehyde (5a) (31.8 mg, $0.300 \mathrm{mmol}, 1.5 \mathrm{eq})$ was then dissolved in dry dichloromethane $(2 \mathrm{~mL})$ and added. The mixture was stirred at room temperature for $16 \mathrm{~h}$ and then filtered over a pad of basic alumina, eluted with ethyl acetate and concentrated under vacuum. The crude oil was purified by column chromatography ( $\mathrm{SiO}_{2}, 8: 2$ Pentane/Ethyl Acetate) affording dimethyl 6-(1,3-dioxoisoindolin-2-yl)-2,5-

12 Some peaks are not resolved in the aromatic region. 
diphenyldihydro-2H-pyran-3,3(4H)-dicarboxylate (6ca) $(53.0 \mathrm{mg}, 0.106 \mathrm{mmol}, 53 \%$ yield) as a white solid.

dr 6:1, diastereoisomeric ratio was calculated in ${ }^{1} \mathrm{H}$ NMR of the isolated product integrating peaks at 4.70 and 4.25 .

$\mathbf{R}_{f} 0.16$ (8:2 Pentane/Ethyl Acetate).

Mp 222-228 ${ }^{\circ} \mathrm{C}$.

${ }^{1} \mathbf{H}$ NMR $\left(400 \mathrm{MHz}, \mathrm{CDCl}_{3}\right)$ on a $5(\mathrm{maj}): 1(\mathrm{~min})$ mixture $\delta 7.94-7.57\left(\mathrm{~m}, 4.8 \mathrm{H}\right.$, Phth maj + min) ${ }^{13} 7.54$ -7.10 (m, $12 \mathrm{H}, 2$ x Ph maj + min), ${ }^{13} 6.34$ (s, $0.2 \mathrm{H}, \mathrm{O}-\mathrm{C}(H)-\mathrm{Ph}$ min), 5.83 (d, J=10.8 Hz, $1 \mathrm{H}, \mathrm{N}-\mathrm{C}(H)-$ O maj), 5.81 (d, $J=10.4 \mathrm{~Hz}, 0.2 \mathrm{H}, \mathrm{N}-\mathrm{C}(H)-\mathrm{O}$ min $) 5.42\left(\mathrm{~s}, 1 \mathrm{H}, \mathrm{O}-\mathrm{C}(H)-\mathrm{Ph}\right.$ maj), ${ }^{13} 4.70$ (ddd, $J=12.8$, 10.9, 4.1 Hz, $1 \mathrm{H}, \mathrm{Ph}-\mathrm{C}(H)-\mathrm{CH}_{2}$ maj), 4.25 (ddd, $J=13.9,10.8,3.3 \mathrm{~Hz}, 0.2 \mathrm{H}, \mathrm{Ph}-\mathrm{C}(H)-\mathrm{CH}_{2}$ min), 3.92 (s, $\left.0.6 \mathrm{H}, \mathrm{OCH}_{3} \min \right),{ }^{13} 3.68\left(\mathrm{~s}, 3 \mathrm{H}, \mathrm{OCH}_{3}\right.$ maj) ${ }^{13} 3.66\left(\mathrm{~s}, 3 \mathrm{H}, \mathrm{OCH}_{3}\right.$ maj), ${ }^{13} 3.34\left(\mathrm{~s}, 0.6 \mathrm{H}, \mathrm{OCH}_{3} \min \right),{ }^{13}$ $2.89\left(\mathrm{dd}, J=13.7,4.2 \mathrm{~Hz}, 1 \mathrm{H}, \mathrm{CH}_{2}\right.$ maj), 2.75 (dd, $J=14.2,3.4 \mathrm{~Hz}, 0.2 \mathrm{H}, \mathrm{CH}_{2}$ min), 2.42 (dd, $J=13.2$, $13.2 \mathrm{~Hz}, 1 \mathrm{H}, \mathrm{CH}_{2}$ maj), ${ }^{13} 2.01-1.91$ (m, $0.6 \mathrm{H}, \mathrm{CH}_{2}$ min).

${ }^{13} \mathbf{C}$ NMR $\left(101 \mathrm{MHz}, \mathrm{CDCl}_{3}\right)$ only maj diastereoisomer characterized $\delta 170.5,168.6,166.9,139.0,138.1$, 134.2, 128.9, 127.9, 127.7, 127.6, 127.5, 127.4, 127.3, 123.5, 83.0, 82.3, 59.1, 52.7, 52.0, 40.7, 39.1.

IR 3064 (w), 3034 (w), 2953 (w), 1723 (s), 1438 (w), 1374 (m), 1254 (m), 1216 (m), 1065 (m).

HRMS (ESI) calcd for $\mathrm{C}_{29} \mathrm{H}_{25} \mathrm{KNO}_{7}{ }^{+}[\mathrm{M}+\mathrm{K}]^{+}$538.1268; found 538.1277.

Diethyl 6-(1,3-dioxoisoindolin-2-yl)-4-methyl-2-phenyldihydro-2H-pyran-3,3(4H)-dicarboxylate (6da)

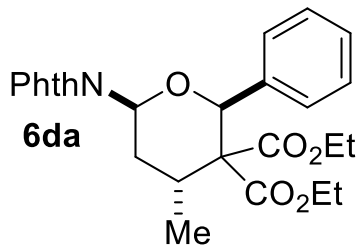

Following the general procedure GP4, diethyl 2-(1,3-dioxoisoindolin-2-yl)-4-methylcyclobutane-1,1dicarboxylate (4d) $(71.9 \mathrm{mg}, 0.200 \mathrm{mmol}, 1 \mathrm{eq})$ and benzaldehyde (5a) $(31.8 \mathrm{mg}, 0.300 \mathrm{mmol}, 1.5 \mathrm{eq})$ were stirred at $0{ }^{\circ} \mathrm{C}$ for $2 \mathrm{~h}$. The crude oil was purified by column chromatography $\left(\mathrm{SiO}_{2}, 8: 2\right.$ Pentane/Ethyl Acetate) affording diethyl 6-(1,3-dioxoisoindolin-2-yl)-4-methyl-2-phenyldihydro-2Hpyran-3,3(4H)-dicarboxylate (6da) $(58.8 \mathrm{mg}, 0.126 \mathrm{mmol}, 63 \%$ yield, $95 \%$ pure $)$ as a white solid.

dr 3:1, diastereoisomeric ratio was calculated in ${ }^{1} \mathrm{H}$ NMR of the isolated product integrating peaks at 5.82 and 5.71 .

The major diastereoisomer is the cis-trans, in analogy with the ${ }^{1} \mathrm{H}$ NMR spectrum of (6db)

$\mathbf{R}_{f} 0.41$ (7:3 Pentane/Ethyl Acetate).

Mp $133-139{ }^{\circ} \mathrm{C}$.

${ }^{1} \mathbf{H}$ NMR $\left(400 \mathrm{MHz}, \mathrm{CDCl}_{3}\right)^{14}$ on a $3(\mathrm{maj}): 1(\mathrm{~min})$ mixture $\delta 7.85(\mathrm{ddd}, J=8.4,5.4,3.1 \mathrm{~Hz}, 2.6 \mathrm{H}$, Phth maj + min), $7.71(\mathrm{ddd}, J=8.5,5.5,3.1 \mathrm{~Hz}, 2.6 \mathrm{H}, \mathrm{Phth}$ maj + min), $7.53-7.43(\mathrm{~m}, 2 \mathrm{H}, \mathrm{Ph}$ maj), 7.33 (m, $0.6 \mathrm{H}, \mathrm{Ph}$ min), $7.25-7.13$ (m, $3.9 \mathrm{H}, \mathrm{Ph}$ maj + min), 5.82 (dd, $J=11.8,3.0 \mathrm{~Hz}, 1 \mathrm{H}, \mathrm{N}-\mathrm{C}(H)-\mathrm{O}$ maj), 5.71 (dd, $J=11.8,2.9 \mathrm{~Hz}, 0.3 \mathrm{H}, \mathrm{N}-\mathrm{C}(H)-\mathrm{O}$ min), 5.48 (s, $1 \mathrm{H}, \mathrm{O}-\mathrm{C}(H)-\mathrm{Ph}$ maj), 5.17 (s, $0.3 \mathrm{H}, \mathrm{O}-\mathrm{C}(H)-$ $\mathrm{Ph}$ min), $4.26-3.90\left(\mathrm{~m}, 5.2 \mathrm{H}, 2 \times \mathrm{OCH}_{2}\right.$ maj + min), 3.69 (dddd, $J=10.7,7.0,7.0,7.0 \mathrm{~Hz}, 1 \mathrm{H}, \mathrm{CH}_{2}$ maj), 3.40 (m, 0.3, $\mathrm{CH}_{2}$ min), ${ }^{17} 3.06-2.94$ (m, $1 \mathrm{H}, \mathrm{Me}-\mathrm{CH}$ maj), ${ }^{17} 2.60-2.48$ (m, $0.3 \mathrm{H}, \mathrm{Me}-\mathrm{CH}$ min) ${ }^{17}$

\footnotetext{
${ }^{13}$ Overlapping with signals of impurities (probably other diastereoisomers present in less than $10 \%$ each).

$1495 \%$ pure (other diastereoisomer not characterized and 5\% impurity).
} 
$1.75-1.63$ (m, $\left.0.3 \mathrm{H}, \mathrm{CH}_{2} \min \right),{ }^{10,17} 1.56$ (ddd, $J=13.4,3.1,3.1 \mathrm{~Hz}, 1 \mathrm{H}, \mathrm{CH}_{2}$ maj), ${ }^{17} 1.26$ (d, $J=7.2 \mathrm{~Hz}$, $3 \mathrm{H}, \mathrm{CH}_{3}$ maj), $1.25\left(\mathrm{~d}, J=6.8 \mathrm{~Hz}, 0.9 \mathrm{H}, \mathrm{CH}_{3} \min \right), 1.20\left(\mathrm{~m}, 3.9 \mathrm{H}, \mathrm{OCH}_{2} \mathrm{CH}_{3}\right.$ maj + min), $1.09(\mathrm{t}, J=$ $\left.7.1 \mathrm{~Hz}, 0.9 \mathrm{H}, \mathrm{OCH}_{2} \mathrm{CH}_{3} \min \right), 0.88$ (t, $J=7.2 \mathrm{~Hz}, 3 \mathrm{H}, \mathrm{OCH}_{2} \mathrm{CH}_{3}$ maj).

${ }^{13} \mathbf{C}$ NMR $\left(101 \mathrm{MHz}, \mathrm{CDCl}_{3}\right)^{12} \delta 170.2,169.5,168.6,167.1,167.0,166.7,138.8,138.5,134.2,131.8$, 128.2 , 127.6, 127.3, 127.2, 127.1, 123.5, 123.5, 83.2, 79.3, 77.2, 76.7, 75.3, 62.9, 61.4, 60.9, 60.7, 60.5, $60.3,39.2,32.8,31.2,30.5,17.5,16.4,14.0,13.7,13.3$.

IR 2982 (w), 2983 (w), 2904 (w), 1780 (w), 1723 (s), 1464 (w), 1373 (s), 1287 (w), 1258 (m), 1230 (m), 1201 (m), 1090 (m), 1048 (m), 1033 (m).

HRMS (ESI) calcd for $\mathrm{C}_{26} \mathrm{H}_{28} \mathrm{NO}_{7}^{+}[\mathrm{M}+\mathrm{H}]^{+} 466.1860$; found 466.1852 .

Diethyl 2-(4-chlorophenyl)-6-(1,3-dioxoisoindolin-2-yl)-4-methyldihydro-2H-pyran-3,3(4H)dicarboxylate (6db)<smiles>CCOC(=O)C1(C)[C@H](c2ccc(Cl)cc2)O[C@H](Nc2ccccc2)C[C@H]1C</smiles>

Following the general procedure GP4, diethyl 2-(1,3-dioxoisoindolin-2-yl)-4-methylcyclobutane-1,1dicarboxylate (4d) $(71.9 \mathrm{mg}, 0.200 \mathrm{mmol}, 1 \mathrm{eq})$ and 4-chlorobenzaldehyde (5b) $(42.2 \mathrm{mg}, 0.300 \mathrm{mmol}$, $1.5 \mathrm{eq})$ were stirred at $0{ }^{\circ} \mathrm{C}$ for $2 \mathrm{~h}$. The crude oil was purified by column chromatography $\left(\mathrm{SiO}_{2}, 8: 2\right.$ Pentane/Ethyl Acetate) affording dimethyl 2-(4-chlorophenyl)-6-(1,3-dioxoisoindolin-2-yl)-5methyldihydro-2H-pyran-3,3(4H)-dicarboxylate (6db) $(81 \mathrm{mg}, 0.162 \mathrm{mmol}, 81 \%$ yield, $90 \%$ pure $)$ as a colorless oil.

dr 3:1, diastereoisomeric ratio was calculated in ${ }^{1} \mathrm{H}$ NMR of the isolated product integrating peaks at 5.80 and 5.69.

The major cis-trans diastereoisomer was characterized. Strong correlations between the signals at 5.80 and 5.44, 5.80 and 1.27-1.17, and 5.44 and 1.27-1.17 were observed. This suggests an axial orientation for the three substituents. In addition, a large coupling constant $(J=12 \mathrm{~Hz})$ of the signal at 5.80 suggests that this proton is in axial position in anti-periplanar relationship with a proton of the neighboring $\mathrm{CH}_{2}$.

$\mathbf{R}_{f} 0.43$ ( $7: 3$ Pentane/Ethyl Acetate).

${ }^{1} \mathbf{H}$ NMR $\left(400 \mathrm{MHz}, \mathrm{CDCl}_{3}\right)^{15}$ on a $3\left(\right.$ maj): $1(\min )$ mixture $\delta 7.92-7.77\left(\mathrm{~m}, 2.6 \mathrm{H}\right.$, Phth maj + min) ${ }^{16}$ 7.77 - 7.65 (m, $2.6 \mathrm{H}$, Phth maj + min), $7.48-7.38$ (m, $2 \mathrm{H}, \mathrm{Ph}$ maj), $7.31-7.23$ (m, 0.6 H, Ph min), ${ }^{11}$ $7.23-7.13(\mathrm{~m}, 2.6 \mathrm{H}, \mathrm{Ph} m a j+\min ),{ }^{16} 5.80(\mathrm{dd}, J=12.0,2.8 \mathrm{~Hz}, 1 \mathrm{H}, \mathrm{N}-\mathrm{C}(H)-\mathrm{O}$ maj), 5.69 (dd, $J=11.8$, $2.9 \mathrm{~Hz}, 0.3 \mathrm{H}, \mathrm{N}-\mathrm{C}(H)-\mathrm{O}$ min $), 5.44(\mathrm{~s}, 1 \mathrm{H}, \mathrm{O}-\mathrm{C}(H)-\mathrm{Ph}$ maj), 5.13 (s, $0.3 \mathrm{H}, \mathrm{O}-\mathrm{C}(H)-\mathrm{Ph}$ min), $4.28-3.89$ $\left(\mathrm{m}, 5.2 \mathrm{H}, 2 \times \mathrm{OCH}_{2} m a j+\right.$ min $), 3.82-3.67\left(\mathrm{~m}, 1 \mathrm{H}, \mathrm{CH}_{2}\right.$ maj), ${ }^{16,17} 3.43-3.30\left(\mathrm{~m}, 0.3 \mathrm{H}, \mathrm{CH}_{2}\right.$ min) ${ }^{17}$ $3.04-2.91$ (m, $1 \mathrm{H}, \mathrm{Me}-\mathrm{CH}$ maj), ${ }^{17} 2.57-2.45$ (m, $0.3 \mathrm{H}, \mathrm{Me}-\mathrm{CH}$ min) ${ }^{17} 1.68$ (ddd, $J=13.5,3.6,3.6 \mathrm{~Hz}$, $0.3 \mathrm{H}, \mathrm{CH}_{2}$ min), ${ }^{17} 1.55$ (ddd, $J=13.4,2.8,2.8 \mathrm{~Hz}, 1 \mathrm{H}, \mathrm{CH}_{2}$ maj), ${ }^{17} 1.27-1.17$ (m, $7.8 \mathrm{H}, 2$ x CH $\mathrm{CH}_{3}$ maj + $\min , 2 \times \mathrm{OCH}_{2} \mathrm{CH}_{3}$ maj + min) $,{ }^{16} 1.12\left(\mathrm{t}, J=7.1 \mathrm{~Hz}, 0.6 \mathrm{H}, \mathrm{OCH}_{2} \mathrm{CH}_{3} \min \right),{ }^{16} 0.94(\mathrm{t}, J=7.2 \mathrm{~Hz}, 3 \mathrm{H}$, $\mathrm{OCH}_{2} \mathrm{CH}_{3}$ maj).

\footnotetext{
$1590 \%$ pure.

${ }^{16}$ Overlapping with signals of another diastereoisomer present in less than $10 \%$ and impurity.

${ }^{17}$ Attributed by HSQC.
} 
${ }^{13} \mathrm{C}$ NMR $\left(101 \mathrm{MHz}, \mathrm{CDCl}_{3}\right)^{18} \delta 170.2,169.4,168.3,167.1,167.0,166.6,137.4,137.1,134.3,134.3$, 133.4, 131.7, 129.7, 128.8, 128.3, 127.8, 127.4, 127.2, 123.6, 82.6, 79.3, 76.5, 75.4, 62.8, 61.5, 61.1, 60.9, $60.7,60.2,39.3,32.8,31.1,30.4,17.6,16.3,14.04,13.98,13.8,13.5$.

IR $2982(\mathrm{w}), 2983(\mathrm{w}), 2904$ (w), 1780 (w), 1723 (s), 1464 (w), 1374 (s), 1287 (w), 1258 (m), 1230 (m), 1201 (m), 1090 (m), 1043 (m), 1033 (m).

HRMS (ESI) calcd for $\mathrm{C}_{26} \mathrm{H}_{26} \mathrm{NO}_{7} \mathrm{ClNa}^{+}[\mathrm{M}+\mathrm{Na}]^{+}$522.1290; found 522.1277.

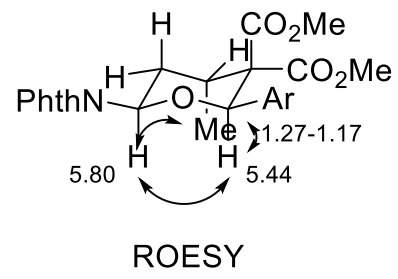

\section{Diastereoisomeric resolution of diethyl 2-(1,3-dioxoisoindolin-2-yl)-4-methylcyclobutane- 1,1-dicarboxylate (86)}

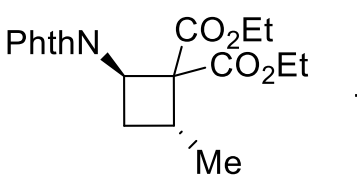

4d<smiles>COc1ccccc1C=O</smiles>

$5 d$

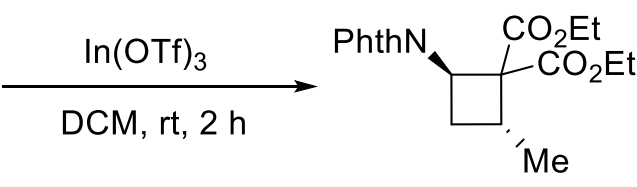

4d

dr 1.1:1

In a glovebox, indium triflate $(22.5 \mathrm{mg}, 0.0400 \mathrm{mmol}, 0.2 \mathrm{eq})$ was added to a microwave vial. The vial was sealed with a Teflon septum and taken out of the glovebox. Dry dichloromethane $(2 \mathrm{~mL})$ was added. Diethyl 2-(1,3-dioxoisoindolin-2-yl)-4-methylcyclobutane-1,1-dicarboxylate (4d) $(71.9 \mathrm{mg}, 0.200 \mathrm{mmol}$, dr 1.1:1, $1 \mathrm{eq})$ was dissolved in dry dichloromethane $(2 \mathrm{~mL})$ and added. 2-Methoxybenzaldehyde (5d) $(22.5 \mathrm{mg}, 0.300 \mathrm{mmol}, 1.5 \mathrm{eq})$ was then dissolved in dry dichloromethane $(2 \mathrm{~mL})$ and added. The reaction was stirred at room temperature for $2 \mathrm{~h}$, and then was filtered over a pad of alumina, eluted with ethyl acetate and concentrated under vacuum. The crude product was purified by column chromatography $\left(\mathrm{SiO}_{2}, 85: 15\right.$ Pentane/Ethyl Acetate) affording diethyl 2-(1,3-dioxoisoindolin-2-yl)-4-methylcyclobutane1,1-dicarboxylate (4d) $(42.4 \mathrm{mg}, 0.117 \mathrm{mmol}$, quantitative yield for the major diastereoisomer) as a pale yellow oil.

dr 14:1, diastereoisomeric ratio was calculated in ${ }^{1} \mathrm{H}$ NMR of the isolated product integrating peaks at 5.58 and 4.91 .

The major trans diastereoisomer was characterized. Strong correlations between the signals at 5.58 and $1.98,5.58$ and 1.14 , and 1.98 and 1.14 were observed.

$\mathbf{R}_{f} 0.44$ (6:4 Pentane/Ethyl Acetate).

${ }^{1} \mathbf{H}$ NMR $\left(400 \mathrm{MHz}, \mathrm{CDCl}_{3}\right)$ only maj diastereoisomer characterized $\delta 7.82(\mathrm{dd}, J=5.4,3.0 \mathrm{~Hz}, 2 \mathrm{H}$, Phth), 7.70 (dd, $J=5.5,3.0 \mathrm{~Hz}, 2 \mathrm{H}, \mathrm{Phth}), 5.58$ (ddd, $J=9.9,8.3,1.2 \mathrm{~Hz}, 1 \mathrm{H}, \mathrm{N}-\mathrm{CH}), 4.21$ (q, $J=7.1$ $\left.\mathrm{Hz}, 2 \mathrm{H}, \mathrm{OCH}_{2}\right),{ }^{19} 3.97\left(\mathrm{~m}, 2 \mathrm{H}, \mathrm{OCH}_{2}\right){ }^{19} 3.65-3.54\left(\mathrm{~m}, 1 \mathrm{H}, \mathrm{CH}_{3}-\mathrm{CH}\right), 3.20$ (ddd, $J=11.7,10.0,8.3$ $\mathrm{Hz}, 1 \mathrm{H}, \mathrm{CH}_{2}$ ), 1.98 (ddd, $\left.J=11.7,10.0,5.4 \mathrm{~Hz}, 1 \mathrm{H}, \mathrm{CH}_{2}\right), 1.23$ (t, $\left.J=7.1 \mathrm{~Hz}, 3 \mathrm{H}, \mathrm{OCH}_{2} \mathrm{CH}_{3}\right), 1.14$ (d, $J$ $\left.=7.3 \mathrm{~Hz}, 3 \mathrm{H}, \mathrm{CH}_{3}\right), 0.90\left(\mathrm{t}, J=7.1 \mathrm{~Hz}, 3 \mathrm{H}, \mathrm{OCH}_{2} \mathrm{CH}_{3}\right)$.

\footnotetext{
${ }^{18}$ One aromatic carbon not resolved.

${ }^{19}$ Overlapping with signals of the minor diastereoisomer.
} 
${ }^{13} \mathrm{C}$ NMR $\left(101 \mathrm{MHz}, \mathrm{CDCl}_{3}\right)$ only maj diastereoisomer characterized $\delta 168.5,168.4,168.1,134.1,131.8$, 123.3, 62.0, 61.7, 61.6, 45.1, 31.1, 28.7, 16.2, 14.2, 13.6.

IR 2980 (w), 2943 (w), $2911(\mathrm{w}), 2876(\mathrm{w}), 1779$ (w), 1719 (s), 1465 (w), 1376 (s), 1335 (w), 1256 (s), $1216(\mathrm{~m}), 1153$ (w), $1116(\mathrm{w}), 1091(\mathrm{w}), 1041(\mathrm{~m})$.

HRMS (ESI) calcd for $\mathrm{C}_{19} \mathrm{H}_{21} \mathrm{NNaO}_{6}{ }^{+}[\mathrm{M}+\mathrm{Na}]^{+}$382.1261; found 382.1265.

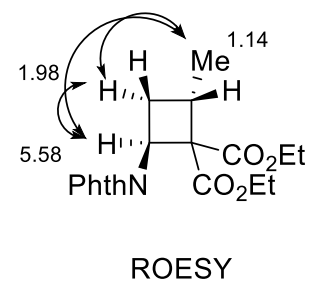

Dimethyl (5-methyl-2,4-dioxo-3,4-dihydropyrimidin-1(2H)-yl)-2-phenyldihydro-2H-pyran-3,3(4H)dicarboxylate (6ea)<smiles>COC(=O)C1(C(C)=O)CC[C@@H](n2cc(C)c(=O)[nH]c2=O)O[C@H]1c1ccccc1</smiles>

Following the general procedure GP5, using benzaldehyde (5a) (46 $\mu \mathrm{L}, 0.45 \mathrm{mmol}, 1.5 \mathrm{eq})$ cyclobutane $4 \mathbf{e}(120 \mathrm{mg}, 0.300 \mathrm{mmol}, 1.0 \mathrm{eq})$, a mixture of diastereoisomers (20:1 by integration of methyl esters at $3.52 \mathrm{ppm}$ and $3.49 \mathrm{ppm})$ 6ea $(102 \mathrm{mg}, 0.255 \mathrm{mmol}, 85 \% \mathrm{NMR} \text { yield })^{\mathbf{2 0}}$ was obtained after column chromatography as a colorless foam. After recrystallization in ethanol, the pure product 6ea (94 mg, 0.23 mmol, 78\% isolated yield) was obtained as a colorless foam and as a mixture of diastereoisomers (> 20:1 by integration of methyl esters at $3.52 \mathrm{ppm}$ and $3.49 \mathrm{ppm}$ ).

$\mathbf{R}_{\boldsymbol{f}}$ 0.27 (3:7 Pentane/Ethyl acetate).

Mp 226.2- $227.9^{\circ} \mathrm{C}$.

${ }^{1}$ H NMR (400 MHz, Chloroform- $d$, major diastereoisomer) $\delta 8.38$ (s, $1 \mathrm{H}, \mathrm{NH}$ ), 7.40 - 7.35 (m, $2 \mathrm{H}, \mathrm{Ar}-$ $\mathrm{H}), 7.34-7.27(\mathrm{~m}, 4 \mathrm{H}$, Ar-H and thymine C=C-H), $5.96(\mathrm{dd}, J=11.1,2.9 \mathrm{~Hz}, 1 \mathrm{H}$, tetrahydropyran$\mathrm{NCH}), 5.23(\mathrm{~s}, 1 \mathrm{H}$, tetrahydropyran- $\mathrm{CH}), 3.68\left(\mathrm{~s}, 3 \mathrm{H}\right.$, ester $\left.\mathrm{CH}_{3}\right), 3.52\left(\mathrm{~s}, 3 \mathrm{H}\right.$, ester $\left.\mathrm{CH}_{3}\right), 2.74-2.64$ $\left(\mathrm{m}, 1 \mathrm{H}\right.$, tetrahydropyran- $\left.\mathrm{CH}_{2}\right), 2.38\left(\mathrm{tdd}, J=12.6,11.0,4.1 \mathrm{~Hz}, 1 \mathrm{H}\right.$, tetrahydropyran- $\left.\mathrm{CH}_{2}\right), 2.25(\mathrm{td}, J=$ $13.4,4.1 \mathrm{~Hz}, 1 \mathrm{H}$, tetrahydropyran- $\left.\mathrm{CH}_{2}\right), 1.99\left(\mathrm{~d}, J=1.2 \mathrm{~Hz}, 3 \mathrm{H}\right.$, thymine- $\left.\mathrm{CH}_{3}\right), 1.97-1.90(\mathrm{~m}, 1 \mathrm{H}$, tetrahydropyran- $\mathrm{CH}_{2}$ ).

${ }^{13}$ C NMR (101 MHz, Chloroform- $d$, major diastereoisomer) $\delta$ 170.3, 169.2, 163.4, 149.9, 137.5, 135.4, $128.5,127.7,127.5,111.6,82.3,82.1,57.1,52.8,52.0,31.6,26.6,12.8$.

IR 3191 (w), 3041 (w), 2954 (w), 2361 (w), 1725 (s), 1693 (s), 1461 (w), 1271 (s), 1096 (w), 1043 (w), $915(\mathrm{w}), 735(\mathrm{~m})$.

HRMS (ESI) calcd for $\mathrm{C}_{20} \mathrm{H}_{22} \mathrm{~N}_{2} \mathrm{NaO}_{7}{ }^{+}[\mathrm{M}+\mathrm{Na}]^{+} 425.1319$, found 425.1324 .

\section{Dimethyl 2-(4-fluorophenyl)-6-(5-methyl-2,4-dioxo-3,4-dihydropyrimidin-1(2H)-yl)dihydro-2H- pyran-3,3(4H)-dicarboxylate (6ef).}

\footnotetext{
${ }^{20}$ Benzamide as impurity which is not possible to separate by column chromatography.
} 
<smiles>COC(=O)C1(C(C)=O)CCC(n2cc(C)c(=O)[nH]c2=O)OC1c1ccc(F)cc1</smiles>

Following the general procedure GP5, using 4-fluorobenzaldehyde (5f) (48 $\mu \mathrm{L}, 0.45 \mathrm{mmol}, 1.5 \mathrm{eq}$ ) cyclobutane $4 \mathbf{e}(120 \mathrm{mg}, 0.300 \mathrm{mmol}, 1.0 \mathrm{eq})$, a mixture of diastereoisomers (12:1 by integration of methyl esters at $3.54 \mathrm{ppm}$ and $3.49 \mathrm{ppm})$ 6ef $(99 \mathrm{mg}, 0.24 \mathrm{mmol}, 79 \% \text { NMR yield })^{20}$ was obtained after column chromatography as a colorless foam. After recrystallization in ethanol, the pure product $\mathbf{6 e f}$ ( 88 $\mathrm{mg}, 0.21 \mathrm{mmol}, 70 \%$ isolated yield) was obtained as a colorless foam and as a mixture of diastereoisomers (12:1 by integration of methyl esters at $3.54 \mathrm{ppm}$ and $3.49 \mathrm{ppm})$.

$\mathbf{R}_{f} 0.30$ (3:7 Pentane/Ethyl acetate).

Mp 94.8- $96.9^{\circ} \mathrm{C}$.

${ }^{1}$ H NMR (400 MHz, Chloroform- $d$, major diastereoisomer) $\delta 8.06$ (s, $\left.1 \mathrm{H}, \mathrm{NH}\right), 7.40-7.33$ (m, $2 \mathrm{H}, \mathrm{Ar}-$ $\mathrm{H}), 7.29(\mathrm{~d}, J=1.3 \mathrm{~Hz}, 1 \mathrm{H}$, thymine $\mathrm{C}=\mathrm{C}-\mathrm{H}), 7.03-6.95(\mathrm{~m}, 2 \mathrm{H}, \mathrm{Ar}-\mathrm{H}), 5.95(\mathrm{dd}, J=11.3,2.9 \mathrm{~Hz}, 1 \mathrm{H}$, tetrahydropyran- $\mathrm{NCH}), 5.21(\mathrm{~s}, 1 \mathrm{H}$, tetrahydropyran- $\mathrm{CH}), 3.69\left(\mathrm{~s}, 3 \mathrm{H}\right.$, ester $\left.\mathrm{CH}_{3}\right), 3.54(\mathrm{~s}, 3 \mathrm{H}$, ester $\left.\mathrm{CH}_{3}\right), 2.74-2.63\left(\mathrm{~m}, 1 \mathrm{H}\right.$, tetrahydropyran- $\left.\mathrm{CH}_{2}\right), 2.48-2.32\left(\mathrm{~m}, 1 \mathrm{H}\right.$, tetrahydropyran- $\left.\mathrm{CH}_{2}\right), 2.32-2.20$ $\left(\mathrm{m}, 1 \mathrm{H}\right.$, tetrahydropyran- $\left.\mathrm{CH}_{2}\right), 1.99\left(\mathrm{~d}, J=1.2 \mathrm{~Hz}, 3 \mathrm{H}\right.$, thymine- $\left.\mathrm{CH}_{3}\right), 1.93(\mathrm{dq}, J=9.3,3.3 \mathrm{~Hz}, 1 \mathrm{H}$, tetrahydropyran- $\mathrm{CH}_{2}$ ).

${ }^{13}$ C NMR (101 MHz, Chloroform- $d$, major diastereoisomer) $\delta$ 170.3, 169.1, 163.6, $162.5(\mathrm{~d}, J=225.5$ $\mathrm{Hz}), 150.2,135.2,133.4(\mathrm{~d}, J=3.3 \mathrm{~Hz}), 129.3(\mathrm{~d}, \mathrm{~J}=8.1 \mathrm{~Hz}), 114.6(\mathrm{~d}, J=21.4 \mathrm{~Hz}), 111.8,82.0,81.6$, $57.0,52.8,52.1,31.4,26.5,12.8$.

IR 3203 (w), 3055 (w), 2961 (w), 2361 (w), 2255 (w), 1724 (s), 1691 (s), 1515 (w), 1464 (w), 1267 (s), 1229 (m), $1100(\mathrm{~m}), 1050(\mathrm{~m}), 913(\mathrm{~m}), 735$ (m).

HRMS (ESI) calcd for $\mathrm{C}^{20} \mathrm{H}^{21} \mathrm{FN}^{2} \mathrm{NaO}^{7+}[\mathrm{M}+\mathrm{Na}]^{+} 443.1225$; found 443.1224 .

\section{Dimethyl 2-(4-methoxyphenyl)-6-(5-methyl-2,4-dioxo-3,4-dihydropyrimidin-1(2H)-yl)dihydro-2H- pyran-3,3(4H)-dicarboxylate (6ec).}<smiles>COc1ccc(C2OC(n3cc(C)c(=O)[nH]c3=O)CCC2(C)OC)cc1</smiles>

Following the general procedure GP5, using $p$-anisaldehyde (5c) $(55 \mu \mathrm{L}, 0.45 \mathrm{mmol}, 1.5 \mathrm{eq})$ cyclobutane $4 \mathbf{e}(120 \mathrm{mg}, 0.300 \mathrm{mmol}, 1.0 \mathrm{eq})$, a mixture of diastereoisomers (5:1 by integration of methyl esters at $3.55 \mathrm{ppm}$ and $3.49 \mathrm{ppm})$ 6ec $(115 \mathrm{mg}, 0.264 \mathrm{mmol}, 88 \% \text { NMR yield })^{20}$ was obtained after column chromatography as a slightly yellow foam. After recrystallization in ethanol, the pure product 6ec $(99 \mathrm{mg}$, $0.22 \mathrm{mmol}, 76 \%$ isolated yield) was obtained as a slightly yellow foam and as a mixture of diastereoisomers (7:1 by integration of methyl esters at $3.55 \mathrm{ppm}$ and $3.49 \mathrm{ppm}$ ).

$\mathbf{R}_{\boldsymbol{f}}$ 0.26 (3:7 Pentane/Ethyl acetate).

Mp 97.4- $99.5^{\circ} \mathrm{C}$. 
${ }^{1} \mathrm{H}$ NMR (400 MHz, Chloroform- $d$, major diastereoisomer) $9.03(\mathrm{~s}, 1 \mathrm{H}, \mathrm{NH}), 7.31(\mathrm{~d}, J=1.3 \mathrm{~Hz}, 1 \mathrm{H}$, thymine $\mathrm{C}=\mathrm{C}-\mathrm{H}), 7.30-7.27(\mathrm{~m}, 2 \mathrm{H}, \mathrm{Ar}-\mathrm{H}), 6.86-6.78(\mathrm{~m}, 2 \mathrm{H}, \mathrm{Ar}-\mathrm{H}), 5.95(\mathrm{dd}, J=11.1,2.9 \mathrm{~Hz}, 1 \mathrm{H}$, tetrahydropyran-NCH), $5.16(\mathrm{~s}, 1 \mathrm{H}$, tetrahydropyran- $\mathrm{CH}), 3.78\left(\mathrm{~s}, 3 \mathrm{H}, \mathrm{O}-\mathrm{Me} \mathrm{CH}_{3}\right), 3.67(\mathrm{~s}, 3 \mathrm{H}$, ester $\left.\mathrm{CH}_{3}\right), 3.55\left(\mathrm{~s}, 3 \mathrm{H}\right.$, ester $\left.\mathrm{CH}_{3}\right), 2.74-2.61\left(\mathrm{~m}, 1 \mathrm{H}\right.$, tetrahydropyran- $\left.\mathrm{CH}_{2}\right), 2.35$ (tdd, $J=12.6,11.0,4.1$ $\mathrm{Hz}, 1 \mathrm{H}$, tetrahydropyran- $\left.\mathrm{CH}_{2}\right), 2.22\left(\mathrm{td}, J=13.4,4.1 \mathrm{~Hz}, 1 \mathrm{H}\right.$, tetrahydropyran- $\left.\mathrm{CH}_{2}\right), 1.97(\mathrm{~d}, J=1.3 \mathrm{~Hz}$, $3 \mathrm{H}$, thymine- $\left.\mathrm{CH}_{3}\right), 1.95-1.88\left(\mathrm{~m}, 1 \mathrm{H}\right.$, tetrahydropyran- $\left.\mathrm{CH}_{2}\right)$.

${ }^{13}$ C NMR (101 MHz, Chloroform- $d$, major diastereoisomer) $\delta 170.3,169.3,163.7,159.5,150.2,135.4$, 129.6, 128.8, 113.0, 111.6, 82.0, 82.0, 57.0, 55.3, 52.7, 52.1, 31.4, 26.6, 12.8.

IR 3191 (w), 3049 (w), 2954 (w), 2839 (w), 2360 (w), 2257 (w), 1687 (s), 1517 (w), 1461 (m), 1255 (s), $1109(\mathrm{~m}), 1044(\mathrm{~m}), 910(\mathrm{~m}), 817(\mathrm{w}), 730(\mathrm{~s})$.

HRMS (ESI) calcd for for $\mathrm{C}_{21} \mathrm{H}_{24} \mathrm{~N}_{2} \mathrm{NaO}_{8}{ }^{+}[\mathrm{M}+\mathrm{Na}]^{+}$455.1425; found 455.1428.

Dimethyl 2-(2-methoxyphenyl)-6-(5-methyl-2,4-dioxo-3,4-dihydropyrimidin-1(2H)-yl)dihydro-2Hpyran-3,3(4H)-dicarboxylate (6ed):

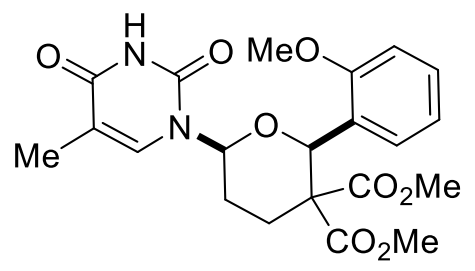

Following the general procedure GP5, using o-anisaldehyde (5d) $(61 \mathrm{mg}, 0.45 \mathrm{mmol}, 1.5 \mathrm{eq})$ cyclobutane $4 \mathbf{e}(120 \mathrm{mg}, 0.300 \mathrm{mmol}, 1.0 \mathrm{eq})$, a mixture of diastereoisomers (10:1 by integration of methyl esters at $3.54 \mathrm{ppm}$ and $3.40 \mathrm{ppm})$ 6ed $(108 \mathrm{mg}, 0.252 \mathrm{mmol}, 84 \% \mathrm{NMR} \text { yield })^{20}$ was obtained after column chromatography as a white solid. After recrystallization in ethanol, the pure product 6ed $(98 \mathrm{mg}, 0.23$ mmol, 76\% isolated yield) was obtained as white solid and as a mixture of diastereoisomers (10:1 by integration of methyl esters at $3.54 \mathrm{ppm}$ and $3.40 \mathrm{ppm})$.

$\mathbf{R}_{f} 0.29$ (3:7 Pentane/Ethyl acetate).

Mp 193.9- $196.2^{\circ} \mathrm{C}$.

${ }^{1}$ H NMR (400 MHz, Chloroform- $d$, major diastereoisomer) $\delta 8.05(\mathrm{~s}, 1 \mathrm{H}, \mathrm{NH}), 7.77$ (dd, $J=7.8,1.7 \mathrm{~Hz}$, $1 \mathrm{H}, \mathrm{Ar}-\mathrm{H}), 7.39(\mathrm{~d}, J=1.4 \mathrm{~Hz}, 1 \mathrm{H}$, thymine $\mathrm{C}=\mathrm{C}-\mathrm{H}), 7.31-7.27(\mathrm{~m}, 1 \mathrm{H}, \mathrm{Ar}-\mathrm{H}), 7.01(\mathrm{td}, J=7.6,1.1$ $\mathrm{Hz}, 1 \mathrm{H}, \mathrm{Ar}-\mathrm{H}), 6.80(\mathrm{dd}, J=8.3,1.1 \mathrm{~Hz}, 1 \mathrm{H}, \mathrm{Ar}-\mathrm{H}), 5.92(\mathrm{dd}, J=11.0,3.0 \mathrm{~Hz}, 1 \mathrm{H}$, tetrahydropyran$\mathrm{NCH}), 5.29$ (s, $1 \mathrm{H}$, tetrahydropyran- $\mathrm{CH}), 3.77\left(\mathrm{~s}, 3 \mathrm{H}, \mathrm{O}-\mathrm{Me} \mathrm{CH}_{3}\right), 3.69\left(\mathrm{~s}, 3 \mathrm{H}\right.$, ester $\left.\mathrm{CH}_{3}\right), 3.54(\mathrm{~s}, 3 \mathrm{H}$, ester $\left.\mathrm{CH}_{3}\right), 2.63-2.55\left(\mathrm{~m}, 2 \mathrm{H}\right.$, tetrahydropyran- $\left.\mathrm{CH}_{2}\right), 2.05-2.01\left(\mathrm{~m}, 1 \mathrm{H}\right.$, tetrahydropyran- $\left.\mathrm{CH}_{2}\right), 1.99$ $\left(\mathrm{d}, J=1.3 \mathrm{~Hz}, 3 \mathrm{H}\right.$, thymine- $\left.\mathrm{CH}_{3}\right), 1.85-1.74\left(\mathrm{~m}, 1 \mathrm{H}\right.$, tetrahydropyran- $\left.\mathrm{CH}_{2}\right)$.

${ }^{13}$ C NMR (101 MHz, Chloroform- $d$, major diastereoisomer) $\delta 169.0,168.9,163.8,156.2,150.0,135.7$, $129.9,129.4,125.6,120.3,111.5,109.4,82.2,76.4,56.9,55.5,52.5,52.3,31.1,27.3,12.9$.

IR 3191 (w), 3045 (w), 2955 (w), 2841 (w), 2361 (w), 2338 (w), 2253 (w), 1731 (s), 1690 (s), 1464 (m), 1377 (w), 1259 (s), 1104 (m), 1047 (m), 914 (m), 733 (s).

HRMS (ESI) calcd for $\mathrm{C}_{21} \mathrm{H}_{24} \mathrm{~N}_{2} \mathrm{NaO}_{8}{ }^{+}[\mathrm{M}+\mathrm{Na}]^{+} 455.1425$; found 455.1429.

\section{Dimethyl 6-(5-methyl-2,4-dioxo-3,4-dihydropyrimidin-1(2H)-yl)-2-(thiophen-2-yl)dihydro-2H- pyran-3,3(4H)-dicarboxylate $(6 \mathrm{eg})$.}




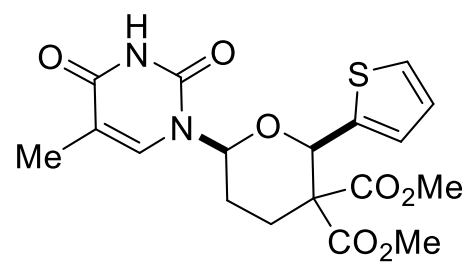

Following the general procedure GP5, using thiophene-2-carbaldehyde (5g) (42 $\mu \mathrm{L}, .45 \mathrm{mmol}, 1.5 \mathrm{eq})$ cyclobutane $4 \mathbf{e}(120 \mathrm{mg}, 0.300 \mathrm{mmol}, 1.0 \mathrm{eq})$, a mixture of diastereoisomers (12:1 by integration of methyl esters at $3.56 \mathrm{ppm}$ and $3.54 \mathrm{ppm}) 6 \mathrm{eg}(111 \mathrm{mg}, 0.270 \mathrm{mmol}, 90 \% \text { NMR yield })^{20}$ was obtained after column chromatography as a white foam. After recrystallization in ethanol, the pure product $\mathbf{6 e g}(97 \mathrm{mg}$, $0.24 \mathrm{mmol}, 79 \%$ isolated yield) was obtained as white foam and as a mixture of diastereoisomers (7:1 by integration of methyl esters at $3.56 \mathrm{ppm}$ and $3.54 \mathrm{ppm}$ ).

$\mathbf{R}_{f} 0.24$ (4:6 Pentane/Ethyl acetate).

Mp $186.4-188.3^{\circ} \mathrm{C}$.

${ }^{1}$ H NMR (400 MHz, Chloroform-d, major diastereoisomer) $\delta 9.74(\mathrm{~s}, 1 \mathrm{H}, \mathrm{NH}), 7.27$ (d, J=1.4 Hz, $1 \mathrm{H}$, , thymine $\mathrm{C}=\mathrm{C}-\mathrm{H}), 7.23(\mathrm{dd}, J=5.1,1.2 \mathrm{~Hz}, 1 \mathrm{H}$, thiophene-H), $7.04(\mathrm{dd}, J=3.5,1.1 \mathrm{~Hz}, 1 \mathrm{H}$, thiophene $\mathrm{H}), 6.92(\mathrm{dd}, J=5.1,3.6 \mathrm{~Hz}, 1 \mathrm{H}$, thiophene $-\mathrm{H}), 5.95(\mathrm{dd}, J=10.9,2.8 \mathrm{~Hz}, 1 \mathrm{H}$, tetrahydropyran-NCH), $5.48\left(\mathrm{~d}, J=0.8 \mathrm{~Hz}, 1 \mathrm{H}\right.$, tetrahydropyran-CH), $3.71\left(\mathrm{~s}, 3 \mathrm{H}\right.$, ester $\left.\mathrm{CH}_{3}\right), 3.56\left(\mathrm{~s}, 3 \mathrm{H}\right.$, ester $\left.\mathrm{CH}_{3}\right), 2.70-$ $2.61\left(\mathrm{~m}, 1 \mathrm{H}\right.$, tetrahydropyran- $\left.\mathrm{CH}_{2}\right), 2.32-2.24\left(\mathrm{~m}, 1 \mathrm{H}\right.$, tetrahydropyran- $\left.\mathrm{CH}_{2}\right), 2.23-2.14(\mathrm{~m}, 1 \mathrm{H}$, tetrahydropyran- $\left.\mathrm{CH}_{2}\right), 1.95\left(\mathrm{~d}, J=1.3 \mathrm{~Hz}, 3 \mathrm{H}\right.$, thymine- $\left.\mathrm{CH}_{3}\right), 1.92(\mathrm{dd}, J=7.9,2.3 \mathrm{~Hz}, 1 \mathrm{H}$, tetrahydropyran- $\mathrm{CH}_{2}$ ).

${ }^{13}$ C NMR (101 MHz, Chloroform-d, major diastereoisomer) $\delta 170.2,168.9,163.6,150.1,139.8,135.2$, $126.2,126.2,125.5,111.7,82.1,79.1,57.4,52.9,52.5,31.1,26.5,12.8$.

IR 3195 (w), 3042 (w), 2954 (w), 2832 (w), 2362 (w), 2252 (w), 2252 (w), 1690 (s), 1441 (w), 1375 (w), $1271(\mathrm{~s}), 1103$ (m), $1050(\mathrm{w}), 913(\mathrm{~m}), 734(\mathrm{~m})$.

HRMS (ESI) calcd for $\mathrm{C}_{18} \mathrm{H}_{20} \mathrm{~N}_{2} \mathrm{NaO}_{7} \mathrm{~S}^{+}[\mathrm{M}+\mathrm{Na}]^{+} 431.0883$; found 431.0879 .

\section{(E)-dimethyl 6-(5-methyl-2,4-dioxo-3,4-dihydropyrimidin-1(2H)-yl)-2-styryldihydro-2H-pyran- 3,3(4H)-dicarboxylate (6ee).}

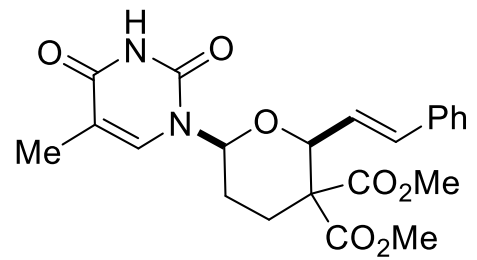

Following the general procedure GP5, using cinnamaldehyde (5e) $(57 \mu \mathrm{L}, .45 \mathrm{mmol}, 1.5 \mathrm{eq})$ cyclobutane $4 \mathbf{e}(120 \mathrm{mg}, 0.300 \mathrm{mmol}, 1.0 \mathrm{eq})$, a mixture of diastereoisomers (2.5:1 by integration of methyl esters at $3.72 \mathrm{ppm}$ and $3.67 \mathrm{ppm}$ ) 6ee (104 mg, $0.243 \mathrm{mmol}, 81 \%$ NMR yield $)^{20}$ was obtained after column chromatography as a yellow foam. After recrystallization in ethanol, the pure product 6ee $(89 \mathrm{mg}, 0.21$ mmol, $69 \%$ isolated yield) was obtained as yellow foam and as a mixture of diastereoisomers (2.5:1 by integration of methyl esters at $3.72 \mathrm{ppm}$ and $3.67 \mathrm{ppm}$ ).

$\mathbf{R}_{\boldsymbol{f}}$ 0.37 (3:7 Pentane/Ethyl acetate).

Mp 73.7-75-5 ${ }^{\circ} \mathrm{C}$. 
${ }^{1}$ H NMR (400 MHz, Chloroform- $d$ ) $\delta 9.03$ (s, 1H, N H, major diastereoisomer), 8.91 (s, $1 \mathrm{H}, \mathrm{NH}$, minor diastereoisomer), $7.39-7.21(\mathrm{~m}, 10 \mathrm{H}, \mathrm{Ar}-\mathrm{H}$, major and minor diastereoisomers), 7.18 (dd, $J=3.4,2.0$ $\mathrm{Hz}, 2 \mathrm{H}$, thymine $\mathrm{C}=\mathrm{C}-\mathrm{H}$, , major and minor diastereoisomers), $6.79(\mathrm{dd}, J=15.7,1.1 \mathrm{~Hz}, 1 \mathrm{H}$, vinyl $\mathrm{CH}$, minor diastereoisomer), $6.61-6.49(\mathrm{~m}, 2 \mathrm{H}$, vinyl $\mathrm{CH}$, major diastereoisomer), 6.31 (dd, $J=15.7,8.0 \mathrm{~Hz}$, $1 \mathrm{H}$, , vinyl $\mathrm{CH}$, minor diastereoisomer), $6.02(\mathrm{dd}, J=11.2,2.8 \mathrm{~Hz}, 1 \mathrm{H}$, tetrahydropyran- $\mathrm{NCH}$, minor diastereoisomer), 5.91 (dd, $J=11.0,3.0 \mathrm{~Hz}, 1 \mathrm{H}$, tetrahydropyran-NCH, major diastereoisomer), 5.40 (d, $J$ $=8.0 \mathrm{~Hz}, 1 \mathrm{H}$, tetrahydropyran- $\mathrm{CH}$, minor diastereoisomer $), 4.68(\mathrm{~d}, \mathrm{~J}=5.3 \mathrm{~Hz}, 1 \mathrm{H}$, tetrahydropyran-CH, major diastereoisomer), $3.87\left(\mathrm{~s}, 3 \mathrm{H}\right.$, ester $\mathrm{CH}_{3}$, minor diastereoisomer), 3.78 (s, $3 \mathrm{H}$, ester $\mathrm{CH}_{3}$, major diastereoisomer), $3.71\left(\mathrm{~s}, 3 \mathrm{H}\right.$, ester $\mathrm{CH}_{3}$, major diastereoisomer), $3.66\left(\mathrm{~s}, 3 \mathrm{H}\right.$, ester $\mathrm{CH}_{3}$, minor diastereoisomer), 2.65 (ddd, $J=13.6,4.2,2.7 \mathrm{~Hz}, 1 \mathrm{H}$, tetrahydropyran- $\mathrm{CH}_{2}$, major diastereoisomer), 2.58 $-2.35\left(\mathrm{~m}, 2 \mathrm{H}\right.$, tetrahydropyran- $\mathrm{CH}_{2}$, minor diastereoisomer), $2.26-2.15\left(\mathrm{~m}, 1 \mathrm{H}\right.$, tetrahydropyran- $\mathrm{CH}_{2}$, major diastereoisomer), $2.15-1.98\left(\mathrm{~m}, 2 \mathrm{H}\right.$, tetrahydropyran- $\mathrm{CH}_{2}$, major diastereoisomer and minor diastereoisomer), 1.95 (d, $J=1.2 \mathrm{~Hz}, 3 \mathrm{H}$, thymine- $\mathrm{CH}_{3}$, major diastereoisomer), 1.93 (d, $J=1.3 \mathrm{~Hz}, 3 \mathrm{H}$, thymine- $\mathrm{CH}_{3}$, minor diastereoisomer), $1.92-1.87\left(\mathrm{~m}, 1 \mathrm{H}\right.$, tetrahydropyran- $\mathrm{CH}_{2}$, major diastereoisomer), $1.56-1.43\left(\mathrm{~m}, 1 \mathrm{H}\right.$, tetrahydropyran- $\mathrm{CH}_{2}$, minor diastereoisomer).

${ }^{13}$ C NMR (101 MHz, Chloroform- $d$ ) $\delta$ 170.0, 169.6, 169.0, 168.3, 163.7, 163.7, 150.2, 150.1, 137.8, 136.5, 135.3, 135.3, 132.7, 128.8, 128.8, 128.6, 128.1, 127.1, 126.8, 125.2, 120.4, 111.6, 111.4, 81.8, 81.7, $76.1,57.0,56.6,53.4,53.2,53.0,52.6,30.7,27.5,26.8,24.1,12.8 .^{21}$

IR 3730 (w), 2955 (w), 2361 (s), 2336 (s), 1744 (m), 1699 (s), 1666 (m), 1442 (w), 1275 (m), 1099 (w), $976(\mathrm{w}), 914(\mathrm{w}), 736(\mathrm{~m})$.

HRMS (ESI) calcd for $\mathrm{C}_{22} \mathrm{H}_{24} \mathrm{~N}_{2} \mathrm{NaO}_{7}^{+}[\mathrm{M}+\mathrm{Na}]^{+}$451.1476; found 451.1474.

\section{Dimethyl (5-fluoro-2,4-dioxo-3,4-dihydropyrimidin-1(2H)-yl)-2-phenyldihydro-2H-pyran-3,3(4H)- dicarboxylate (6fa)}

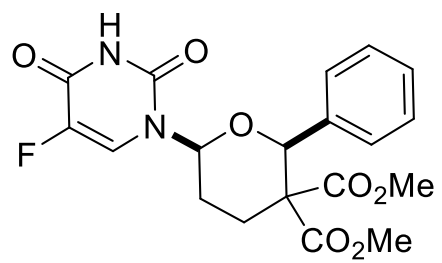

Following the general procedure GP5, using benzaldehyde (5a) $(46 \mu \mathrm{L}, 0.45 \mathrm{mmol}, 1.5 \mathrm{eq})$ and cyclobutane $4 \mathbf{f}(121 \mathrm{mg}, 0.300 \mathrm{mmol}, 1.0 \mathrm{eq})$, a mixture of diastereoisomers (13:1 by integration of methyl esters at $3.52 \mathrm{ppm}$ and $3.48 \mathrm{ppm}) \mathbf{6 f a}\left(78 \mathrm{mg}, 0.19 \mathrm{mmol}, 64 \% \mathrm{NMR}\right.$ yield ${ }^{20}$ was obtained after column chromatography as a white foam. After recrystallization in ethanol, the pure product $\mathbf{6} \mathbf{f a}(68 \mathrm{mg}$, $0.17 \mathrm{mmol}, 56 \%$ yield) was obtained as white foam and as a mixture of diastereoisomers ( $>20: 1$ by integration of methyl esters at $3.52 \mathrm{ppm}$ and $3.48 \mathrm{ppm})$.

$\mathbf{R}_{f}$ 0.32 (5:5 Pentane/Ethyl acetate).

Mp 80.1- $81.7^{\circ} \mathrm{C}$.

${ }^{1}$ H NMR (400 MHz, Chloroform-d, major diastereoisomer) $\delta 9.95$ (s, $\left.1 \mathrm{H}, \mathrm{NH}\right), 7.63$ (d, $J=5.8 \mathrm{~Hz}, 1 \mathrm{H}$, Ar-H), $7.39-7.34$ (m, 2 H, Ar-H), $7.33-7.27$ (m, $3 \mathrm{H}$, Ar-H and F-uracil C=C-H), $6.01-5.93(\mathrm{~m}, 1 \mathrm{H}$, tetrahydropyran- $\mathrm{NCH}), 5.23(\mathrm{~s}, 1 \mathrm{H}$, tetrahydropyran- $\mathrm{CH}), 3.67\left(\mathrm{~s}, 3 \mathrm{H}\right.$, ester $\left.\mathrm{CH}_{3}\right), 3.52(\mathrm{~s}, 3 \mathrm{H}$, ester $\left.\mathrm{CH}_{3}\right), 2.71-2.62\left(\mathrm{~m}, 1 \mathrm{H}\right.$, tetrahydropyran- $\left.\mathrm{CH}_{2}\right), 2.40-2.19\left(\mathrm{~m}, 2 \mathrm{H}\right.$, tetrahydropyran- $\left.\mathrm{CH}_{2}\right), 2.02-1.95$ $\left(\mathrm{m}, 1 \mathrm{H}\right.$, tetrahydropyran- $\left.\mathrm{CH}_{2}\right)$.

\footnotetext{
213 carbons of the minor diastereoisomer are not resolved.
} 
${ }^{13}$ C NMR (101 MHz, Chloroform-d, major diastereoisomer) $\delta 170.1,169.1,156.98(\mathrm{~d}, J=26.6 \mathrm{~Hz})$, $148.9,140.93(\mathrm{~d}, J=238.5 \mathrm{~Hz}), 137.2,128.5,127.7,127.4,124.12(\mathrm{~d}, J=33.6 \mathrm{~Hz}), 82.2,82.1,56.9,52.8$, $52.1,31.2,26.5$.

IR 3204 (w), 3064 (w), 2955 (w), 2361 (m), 2257 (w), 1717 (s), 1457 (w), 1368 (w), 1265 (s), 1103 (m), $1043(\mathrm{w}), 912(\mathrm{~s}), 731$ (s).

HRMS (ESI) calcd for $\mathrm{C}_{19} \mathrm{H}_{19} \mathrm{FN}_{2} \mathrm{NaO}_{7}^{+}[\mathrm{M}+\mathrm{Na}]^{+} 429.1068$; found 429.1065 .

\section{Dimethyl -2-(tert-butyldimethylsilyl)oxy)-4-(1,3-dioxoisoindolin-2-yl)-2-phenylcyclohexane-1,1- dicarboxylate (8aa)}

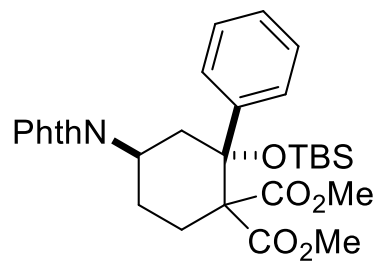

Following the general procedure GP6, using dimethyl 2-(1,3-dioxoisoindolin-2-yl)cyclobutane-1,1dicarboxylate (4a) $(63.5 \mathrm{mg}, 0.200 \mathrm{mmol}, 1 \mathrm{eq})$ and tert-butyldimethyl(1-phenylvinyl)oxy)silane (7a) (70.4 mg, $0.300 \mathrm{mmol}, 1.5 \mathrm{eq}$ ), dimethyl -2-(tert-butyldimethylsilyl)oxy)-4-(1,3-dioxoisoindolin-2-yl)-2phenylcyclohexane-1,1-dicarboxylate (8aa) (105 mg, $0.190 \mathrm{mmol}, 95 \%$ yield) was obtained as a colorless solid.

Mp 188.8-190.0 ${ }^{\circ} \mathrm{C}$.

${ }^{1} \mathbf{H}$ NMR (400 MHz, $\left.\mathrm{CDCl}_{3}\right) \delta 7.83(\mathrm{dd}, J=5.5,3.0 \mathrm{~Hz}, 2 \mathrm{H}, \mathrm{Phth}), 7.72$ (dd, $\left.J=5.4,3.1 \mathrm{~Hz}, 2 \mathrm{H}, \mathrm{Phth}\right)$, $7.44-7.38$ (m, $2 \mathrm{H}, \mathrm{Ar}), 7.26-7.21$ (m, $3 \mathrm{H}, \mathrm{Ar}), 4.86$ (tt, $J=12.5,4.7 \mathrm{~Hz}, 1 \mathrm{H}, \mathrm{N}-\mathrm{C}-\mathrm{H}), 3.96$ (dd, $J=$ 13.6, $\left.12.6 \mathrm{~Hz}, 1 \mathrm{H}, \mathrm{CH}_{2}\right), 3.66$ (s, $\left.3 \mathrm{H}, \mathrm{OMe}\right), 3.64$ (s, $\left.3 \mathrm{H}, \mathrm{OMe}\right), 2.91\left(\mathrm{td}, J=14.2,3.7 \mathrm{~Hz}, 1 \mathrm{H}, \mathrm{CH}_{2}\right.$ cyclohexane), 2.31 (dt, $\left.J=13.8,3.5 \mathrm{~Hz}, 1 \mathrm{H}, \mathrm{CH}_{2}\right), 2.23-2.01\left(\mathrm{~m}, 2 \mathrm{H}, \mathrm{CH}_{2}\right.$ cyclohexane), $1.88-1.74$ (m, $1 \mathrm{H}, \mathrm{CH}_{2}$ cyclohexane), 1.06 (s, $\left.9 \mathrm{H}, \mathrm{Si}-{ }^{t} \mathrm{Bu}\right), 0.14$ (s, $\left.3 \mathrm{H}, \mathrm{Si}-\mathrm{Me}\right),-0.55$ (s, $3 \mathrm{H}, \mathrm{Si}-\mathrm{Me}$ ).

${ }^{13}$ C NMR $\left(101 \mathrm{MHz}, \mathrm{CDCl}_{3}\right) \delta 170.1,169.9,168.4,143.0,134.1,132.1,128.8,127.8,126.5,123.3,79.7$, 64.6, 52.3, 52.0, 46.6, 36.7, 29.4, 26.4, 25.3, 19.3, -1.3, -2.5.

IR 2953 (w), 2890 (w), 2856 (w), 2361 (w), 2339 (w), 1735 (s), 1714 (s), 1373 (m), 1274 (m), 1245 (m), 1039 (m), $1038(\mathrm{~m})$.

The data for 8 aa matches the reported values. ${ }^{5}$

Dimethyl-2-(tert-butyldimethylsilyl)oxy)-4-(1,3-dioxoisoindolin-2-yl)-2-(4methoxyphenyl)cyclohexane-1,1-dicarboxylate (8ab)

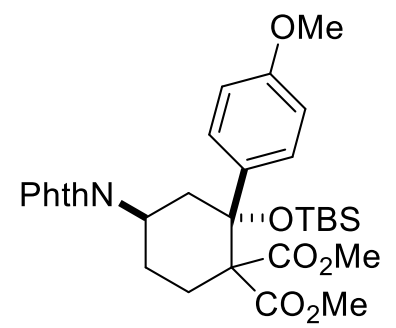

Following the general procedure GP6, using dimethyl 2-(1,3-dioxoisoindolin-2-yl)cyclobutane-1,1dicarboxylate (4a) $\quad(65.5 \quad \mathrm{mg}, \quad 0.200 \quad \mathrm{mmol}, \quad 1 \quad$ eq) and tert-butyl(1-(4methoxyphenyl)vinyl)oxy)dimethylsilane (7b) $(79.0 \mathrm{mg}, 0.300 \mathrm{mmol}, 1.5 \mathrm{eq})$, dimethyl -2-(tert- 
butyldimethylsilyl)oxy)-4-(1,3-dioxoisoindolin-2-yl)-2-(4-methoxyphenyl)cyclohexane-1,1-dicarboxylate (8ab) (105.2 mg, $0.181 \mathrm{mmol}, 90 \%)$ was obtained as a colorless solid.

${ }^{1} \mathbf{H}$ NMR $\left(400 \mathrm{MHz}, \mathrm{CDCl}_{3}\right) \delta 7.83(\mathrm{dd}, J=5.5,3.0 \mathrm{~Hz}, 2 \mathrm{H}, \mathrm{Ar} \mathrm{Phth}), 7.71(\mathrm{dd}, J=5.4,3.1 \mathrm{~Hz}, 2 \mathrm{H}, \mathrm{Ar}$ Phth), $7.34-7.29$ (m, 2 H, Ar), $6.91-6.64$ (m, 2 H, Ar), $5.05-4.69$ (m, $1 \mathrm{H}, \mathrm{N}-\mathrm{C}-\mathrm{H}), 3.92$ (dd, $J=13.5$, $12.6 \mathrm{~Hz}, 1 \mathrm{H}, \mathrm{CH}_{2}$ ), 3.79 (s, $3 \mathrm{H}, \mathrm{OMe}$ ), 3.67 (s, $3 \mathrm{H}, \mathrm{OMe}$ ), 3.64 (s, $3 \mathrm{H}, \mathrm{OMe}$ ), 2.89 (td, J = 14.2, $3.7 \mathrm{~Hz}$, $\left.1 \mathrm{H}, \mathrm{CH}_{2}\right), 2.30$ (dt, $\left.J=13.8,3.5 \mathrm{~Hz}, 1 \mathrm{H}, \mathrm{CH}_{2}\right), 2.22-1.96\left(\mathrm{~m}, 2 \mathrm{H}, \mathrm{CH}_{2}\right), 1.90-1.69\left(\mathrm{~m}, 1 \mathrm{H}, \mathrm{CH}_{2}\right)$, 1.05 (s, 9 H, OTBS), 0.14 (s, 3 H, OTBS), -0.50 (s, 3 H, OTBS).

${ }^{13}$ C NMR $\left(101 \mathrm{MHz}, \mathrm{CDCl}_{3}\right) \delta 171.5,171.2,169.7,160.12,136.5,135.4,133.4,131.2,124.6,113.0$, $80.7,65.8,56.5,53.6,53.2,47.8,38.2,30.7,27.7,26.6,20.6,-0.0,-1.1$.

IR 2953 (w), 2933 (w), 2856 (w), 1731 (s), 1712 (s), 1612 (w), 1373 (m), 1373 (m), 1254 (m), 1037 (s), $840(\mathrm{~m})$.

HRMS (ESI) calcd for $\mathrm{C}_{31} \mathrm{H}_{40} \mathrm{NO}_{8} \mathrm{Si}^{+}[\mathrm{M}+\mathrm{H}]^{+}$582.2518; found 582.2500.

\section{Dimethyl-2-(tert-butyldimethylsilyl)oxy)-4-(1,3-dioxoisoindolin-2-yl)-2-(4-fluorophenyl)cyclohexane-} 1,1-dicarboxylate (8ac)

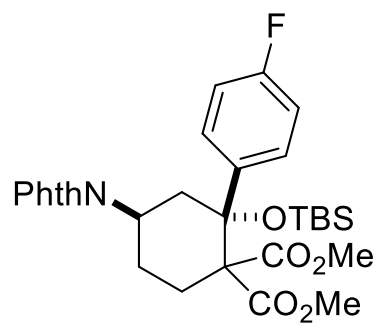

Following the general procedure GP6, using dimethyl 2-(1,3-dioxoisoindolin-2-yl)cyclobutane-1,1dicarboxylate (4a) $(63.5 \mathrm{mg}, 0.200 \mathrm{mmol}, 1 \mathrm{eq})$ and tert-butyl(1-(4-fluorophenyl)vinyl)oxy)dimethylsilane (7c) $(76.0 \mathrm{mg}, 0.300 \mathrm{mmol}, 1.5 \mathrm{eq})$, dimethyl -2-(tert-butyldimethylsilyl)oxy)-4-(1,3-dioxoisoindolin-2yl)-2-(4-fluorophenyl)cyclohexane-1,1-dicarboxylate (8ac) (113 mg, $0.198 \mathrm{mmol}, 99 \%)$ was obtained as a colorless solid.

${ }^{1} \mathbf{H}$ NMR $\left(400 \mathrm{MHz}, \mathrm{CDCl}_{3}\right) \delta 7.83(\mathrm{dd}, J=5.5,3.0 \mathrm{~Hz}, 2 \mathrm{H}, \mathrm{Ar} \mathrm{Phth}), 7.72(\mathrm{dd}, J=5.4,3.0 \mathrm{~Hz}, 2 \mathrm{H}, \mathrm{Ar}$ Phth), $7.48-7.34$ (m, 2 H, Ar), 6.93 (dd, $J=9.3,8.2 \mathrm{~Hz}, 2 \mathrm{H}, \mathrm{Ar}), 4.84$ (tt, $J=12.6,4.6 \mathrm{~Hz}, 1 \mathrm{H}, \mathrm{N}-\mathrm{C}-\mathrm{H}$ ), 3.97 (dd, $J=13.6,12.6 \mathrm{~Hz}, 1 \mathrm{H}, \mathrm{CH}_{2}$ ), 3.67 (s, $3 \mathrm{H}, \mathrm{OMe}$ ), 3.64 (s, $\left.3 \mathrm{H}, \mathrm{OMe}\right), 2.88$ (td, $J=14.2,3.7 \mathrm{~Hz}$, $\left.1 \mathrm{H}, \mathrm{CH}_{2}\right), 2.31\left(\mathrm{dt}, J=13.9,3.4 \mathrm{~Hz}, 1 \mathrm{H}, \mathrm{CH}_{2}\right), 2.22-1.94\left(\mathrm{~m}, 2 \mathrm{H}, \mathrm{CH}_{2}\right), 1.91-1.74\left(\mathrm{~m}, 1 \mathrm{H}, \mathrm{CH}_{2}\right)$, 1.05 (s, 9 H, OTBS), 0.17 (s, 3 H, OTBS), -0.51 (s, 3 H, OTBS).

${ }^{13}$ C NMR $\left(101 \mathrm{MHz}, \mathrm{CDCl}_{3}\right) \delta 169.8,169.7,168.3,134.0,132.0,130.5(\mathrm{~d}, J=8.0 \mathrm{~Hz}), 123.2,113.1(\mathrm{~d}, J$ $=21.1 \mathrm{~Hz}), 79.2,64.4,52.2,51.8,46.3,36.7,29.2,26.2,25.1,19.1,-1.4,-2.5 .^{22}$

IR 2953 (w), 2952 (w), 2857 (w), 2856 (w), 1735 (s), 1715 (s), 1375 (m), 1245 (m), 1244 (m), 1039 (m).

HRMS (ESI) calcd for $\mathrm{C}_{30} \mathrm{H}_{36} \mathrm{FNNaO}_{7} \mathrm{Si}^{+}[\mathrm{M}+\mathrm{Na}]^{+}$592.2137; found 592.2142.

\section{Dimethyl-2-(tert-butyldimethylsilyl)oxy)-4-(1,3-dioxoisoindolin-2-yl)-2-(4- (methoxycarbonyl)phenyl)cyclohexane-1,1-dicarboxylate (8ad)}

\footnotetext{
${ }^{22}$ Carbon with fluoride not resolved.
} 


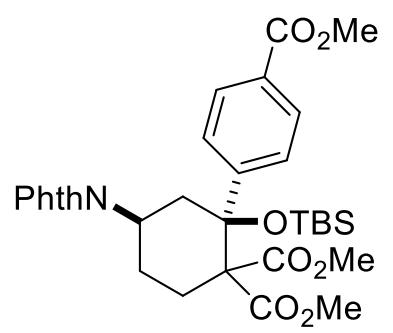

Following the general procedure GP6, using dimethyl 2-(1,3-dioxoisoindolin-2-yl)cyclobutane-1,1dicarboxylate (4a) $\quad(63.5 \mathrm{mg}, \quad 0.200 \mathrm{mmol}, 1 \mathrm{eq})$ and methyl 4-(1-(tertbutyldimethylsilyl)oxy)vinyl)benzoate (7d) $(88.0 \mathrm{mg}, 0.300 \mathrm{mmol}, 1.5 \mathrm{eq})$. A mixture of two diastereomers of dimethyl -2-(tert-butyldimethylsilyl)oxy)-4-(1,3-dioxoisoindolin-2-yl)-2-(4methoxycarbonyl)phenyl)cyclohexane-1,1-dicarboxylate (8ad) $(99.6 \mathrm{mg}, 0.163 \mathrm{mmol}, 82 \%)$ was obtained as a colorless solid.

$\mathrm{dr}=2: 1$, Diastereoisomeric ratio was calculated in ${ }^{1} \mathrm{H}$ crude NMR integrating peaks at 0.17 and 0.12 . Analysis of isolated fractions of the chromatography column allowed the characterization of major cis diastereomer.

${ }^{1}$ H NMR $\left(400 \mathrm{MHz}, \mathrm{CDCl}_{3}\right) \delta 7.97-7.91$ (m, $\left.2 \mathrm{H}, \mathrm{Ar}\right), 7.84$ (dd, $J=5.4,3.0 \mathrm{~Hz}, 2 \mathrm{H}$, Ar Phth), 7.72 (dd, $J=5.4,3.0 \mathrm{~Hz}, 2 \mathrm{H}$, Ar Phth), $7.55-7.48$ (m, $2 \mathrm{H}, \mathrm{Ar}), 4.85$ (tt, $J=12.5,4.7 \mathrm{~Hz}, 1 \mathrm{H}, \mathrm{N}-\mathrm{C}-\mathrm{H}), 3.99$ (dd, $\left.J=13.6,12.6 \mathrm{~Hz}, 1 \mathrm{H}, \mathrm{CH}_{2}\right), 3.90(\mathrm{~s}, 3 \mathrm{H}, \mathrm{OMe}), 3.66(\mathrm{~s}, 3 \mathrm{H}, \mathrm{OMe}), 3.63(\mathrm{~s}, 3 \mathrm{H}, \mathrm{OMe}), 2.90(\mathrm{td}, J=$ $\left.14.2,3.7 \mathrm{~Hz}, 1 \mathrm{H}, \mathrm{CH}_{2}\right), 2.32\left(\mathrm{dt}, J=13.8,3.4 \mathrm{~Hz}, 1 \mathrm{H}, \mathrm{CH}_{2}\right), 2.21-2.01\left(\mathrm{~m}, 2 \mathrm{H}, \mathrm{CH}_{2}\right), 1.83(\mathrm{dd}, J=$ 13.1, 3.5 Hz, $1 \mathrm{H}, \mathrm{CH}_{2}$ ), 1.05 (s, $9 \mathrm{H}, \mathrm{OTBS}$ ), 0.17 (s, $3 \mathrm{H}$, OTBS), -0.56 (s, $3 \mathrm{H}$, OTBS).

${ }^{13} \mathrm{C}$ NMR $\left(101 \mathrm{MHz}, \mathrm{CDCl}_{3}\right) \delta 169.6,169.6,168.3,166.9,148.1,134.0,131.9,129.2,128.8,127.6$, $123.2,79.4,64.4,52.2,52.0,51.9,46.2,36.5,29.2,26.2,25.1,19.1,-1.4,-2.5$.

IR 2955 (w), 2955 (w), 2902 (w), 2902 (w), 2901 (w), 2901 (w), 2860 (w), 1716 (s), 1375 (m), 1375 (m), $1281(\mathrm{~m}), 1281(\mathrm{~m}), 1246(\mathrm{w}), 1040(\mathrm{~m}), 1040(\mathrm{~m})$.

HRMS (ESI) calcd for $\mathrm{C}_{32} \mathrm{H}_{39} \mathrm{NNaO}_{9} \mathrm{Si}^{+}[\mathrm{M}+\mathrm{Na}]^{+}$632.2286; found 632.2282.

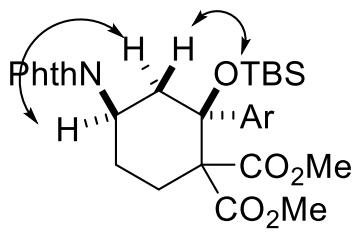

ROESY

Dimethyl-2-(tert-butyldimethylsilyl)oxy)-4-(1,3-dioxoisoindolin-2-yl)-3-methyl-2-phenylcyclohexane1,1-dicarboxylate (8ae)

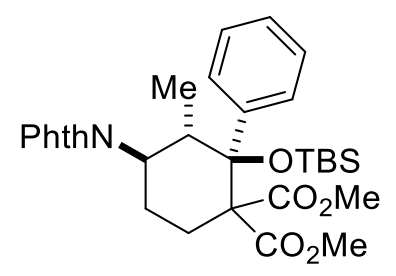

Following the general procedure GP6, using dimethyl 2-(1,3-dioxoisoindolin-2-yl)cyclobutane-1,1dicarboxylate (4a) $(63.5 \mathrm{mg}, 0.200 \mathrm{mmol}, 1 \mathrm{eq})$ and (Z)-tert-butyldimethyl(1-phenylprop-1-en-1yl)oxy)silane (7e) $(74.6 \mathrm{mg}, 0.300 \mathrm{mmol}, 1.5 \mathrm{eq})$, a mixture of two diastereomers of dimethyl -2-(tertbutyldimethylsilyl)oxy)-4-(1,3-dioxoisoindolin-2-yl)-3-methyl-2-phenylcyclohexane-1,1-dicarboxylate (8ae) (102 mg, $0.181 \mathrm{mmol}, 90 \%)$ was obtained as a colorless solid. 
$\mathrm{dr}=2: 1$, Diastereoisomeric ratio was calculated in ${ }^{1} \mathrm{H}$ crude NMR integrating peaks at 4.75 and 4.33 . Analysis of isolated fractions of the chromatography column allowed the characterization of each diastereomer.

Major cis-diastereoisomer

${ }^{1} \mathbf{H}$ NMR (400 MHz, $\left.\mathrm{CDCl}_{3}\right) \delta 7.99$ - 7.86 (m, $2 \mathrm{H}$, Ar Phth), 7.86 - 7.73 (m, $2 \mathrm{H}$, Ar Phth), 7.70 - 7.59 $(\mathrm{m}, 2 \mathrm{H}, \mathrm{Ar}), 7.32-7.24(\mathrm{~m}, 3 \mathrm{H}, \mathrm{Ar}), 4.62(\mathrm{td}, J=12.0,4.7 \mathrm{~Hz}, 1 \mathrm{H}, \mathrm{N}-\mathrm{C}-\mathrm{H}), 4.38(\mathrm{dq}, J=11.5,6.8 \mathrm{~Hz}$, $1 \mathrm{H}, \mathrm{CH}-\mathrm{Me}$ ), 3.71 (s, $3 \mathrm{H}, \mathrm{OMe}), 3.53$ (s, $3 \mathrm{H}, \mathrm{OMe}), 3.03-2.80\left(\mathrm{~m}, 1 \mathrm{H}, \mathrm{CH}_{2}\right), 2.42-2.25(\mathrm{~m}, 2 \mathrm{H}$, $\mathrm{CH}_{2}$ ), $1.93-1.80\left(\mathrm{~m}, 1 \mathrm{H}, \mathrm{CH}_{2}\right), 1.17$ (s, $\left.9 \mathrm{H}, \mathrm{OTBS}\right), 0.92$ (d, $\left.J=6.8 \mathrm{~Hz}, 3 \mathrm{H}, \mathrm{CH}_{3}\right), 0.42(\mathrm{~s}, 3 \mathrm{H}$, OTBS), 0.00 (s, $3 \mathrm{H}$, OTBS).

${ }^{13} \mathbf{C ~ N M R}^{23}\left(101 \mathrm{MHz}, \mathrm{CDCl}_{3}\right) \delta 169.8,169.7,168.6,140.7,134.1,132.3,131.6,129.7,127.2,123.4$, $85.5,65.8,52.2,52.2,51.5,36.3,29.4,26.6,25.4,20.4,13.6,-0.1,-1.6$.

IR 2951 (w), 2950 (w), 2857 (w), 1736 (s), 1713 (s), 1372 (m), 1371 (m), 1276 (m), 1240 (m), 1134 (m), $1051(\mathrm{~m}), 836(\mathrm{w})$.

HRMS (ESI) calcd for $\mathrm{C}_{31} \mathrm{H}_{40} \mathrm{NO}_{7} \mathrm{Si}^{+}[\mathrm{M}+\mathrm{H}]^{+}$566.2569; found 566.2563.

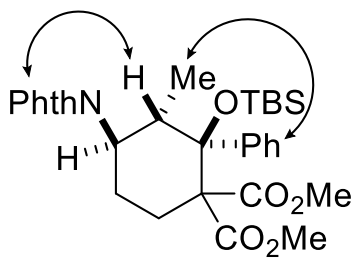

ROESY

Minor trans-diastereoisomer

${ }^{1} \mathbf{H}$ NMR $\left(400 \mathrm{MHz}, \mathrm{CDCl}_{3}\right) \delta 8.11-8.01$ (m, $2 \mathrm{H}, \mathrm{Ar}$ Phth), 7.81 (ddd, $J=7.7,1.3,0.7 \mathrm{~Hz}, 1 \mathrm{H}, \mathrm{Ar}$ ), 7.63 (ddd, $J=7.0,1.6,0.7 \mathrm{~Hz}, 1 \mathrm{H}, \mathrm{Ar}), 7.56-7.37$ (m, $5 \mathrm{H}, \mathrm{Ar}+$ Ar Phth), 4.76 (dd, $J=9.4,7.2 \mathrm{~Hz}, 1$ H, CH-Me), 4.15 (ddd, $J=12.3,9.4,2.9 \mathrm{~Hz}, 1 \mathrm{H}, \mathrm{N}-\mathrm{C}-\mathrm{H}$ ), 3.87 (s, $3 \mathrm{H}, \mathrm{OMe}$ ), 3.40 (s, $3 \mathrm{H}, \mathrm{OMe}$ ), 2.88 $\left(\mathrm{td}, J=14.2,3.9 \mathrm{~Hz}, 1 \mathrm{H}, \mathrm{CH}_{2}\right), 2.42\left(\mathrm{dt}, J=14.2,3.4 \mathrm{~Hz}, 1 \mathrm{H}, \mathrm{CH}_{2}\right), 2.00(\mathrm{dd}, J=13.3,3.3 \mathrm{~Hz}, 1 \mathrm{H}$, $\mathrm{CH}_{2}$ ), $1.63-1.46\left(\mathrm{~m}, 1 \mathrm{H}, \mathrm{CH}_{2}\right), 1.18\left(\mathrm{~d}, J=7.2 \mathrm{~Hz}, 3 \mathrm{H}, \mathrm{CH}_{3}\right), 0.94$ (s, $\left.9 \mathrm{H}, \mathrm{OTBS}\right),-0.03$ (s, $3 \mathrm{H}$, OTBS), -0.34 (s, 3 H, OTBS).

${ }^{13} \mathbf{C}$ NMR the sample was degraded and a mixture of the product and a side product was present (probably the open product as there is a peak at $200 \mathrm{ppm})$.

IR 2955 (w), 2859 (w), 1740 (m), 1703 (s), 1702 (s), 1329 (w), 1272 (m), 1221 (w), 1093 (m), 1072 (m), $863(\mathrm{w}), 842(\mathrm{w})$.

HRMS (ESI). calcd for $\mathrm{C}_{31} \mathrm{H}_{40} \mathrm{NO}_{7} \mathrm{Si}^{+}[\mathrm{M}+\mathrm{H}]^{+}$566.2569; found 566.2568.

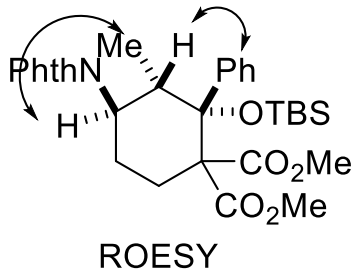

${ }^{23}$ Peaks of impurities are present in the aromatic area. 
5. Spectra of New Compounds 


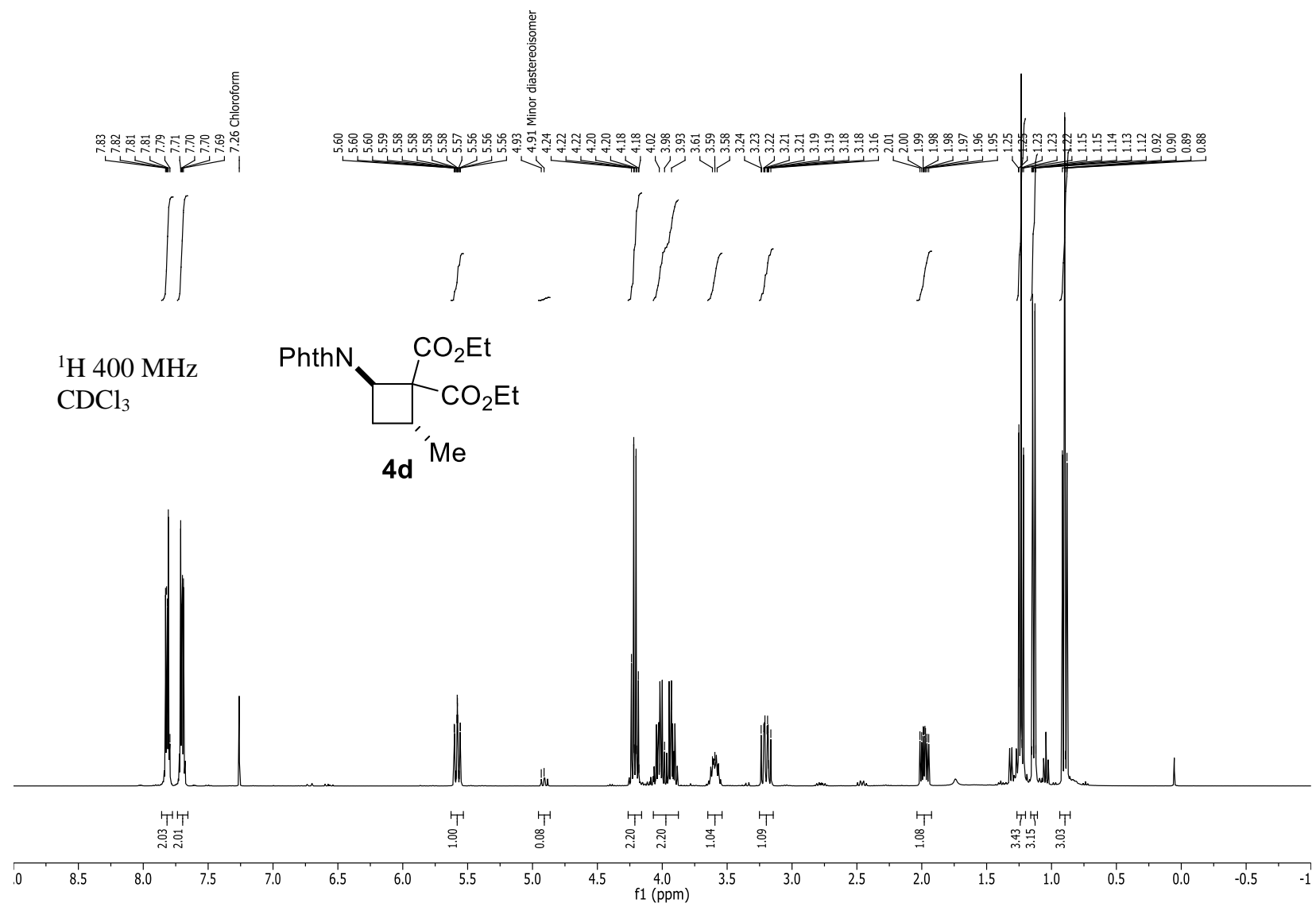

${ }^{13} \mathrm{C} 100 \mathrm{MHz}$

$\mathrm{CDCl}_{3}$

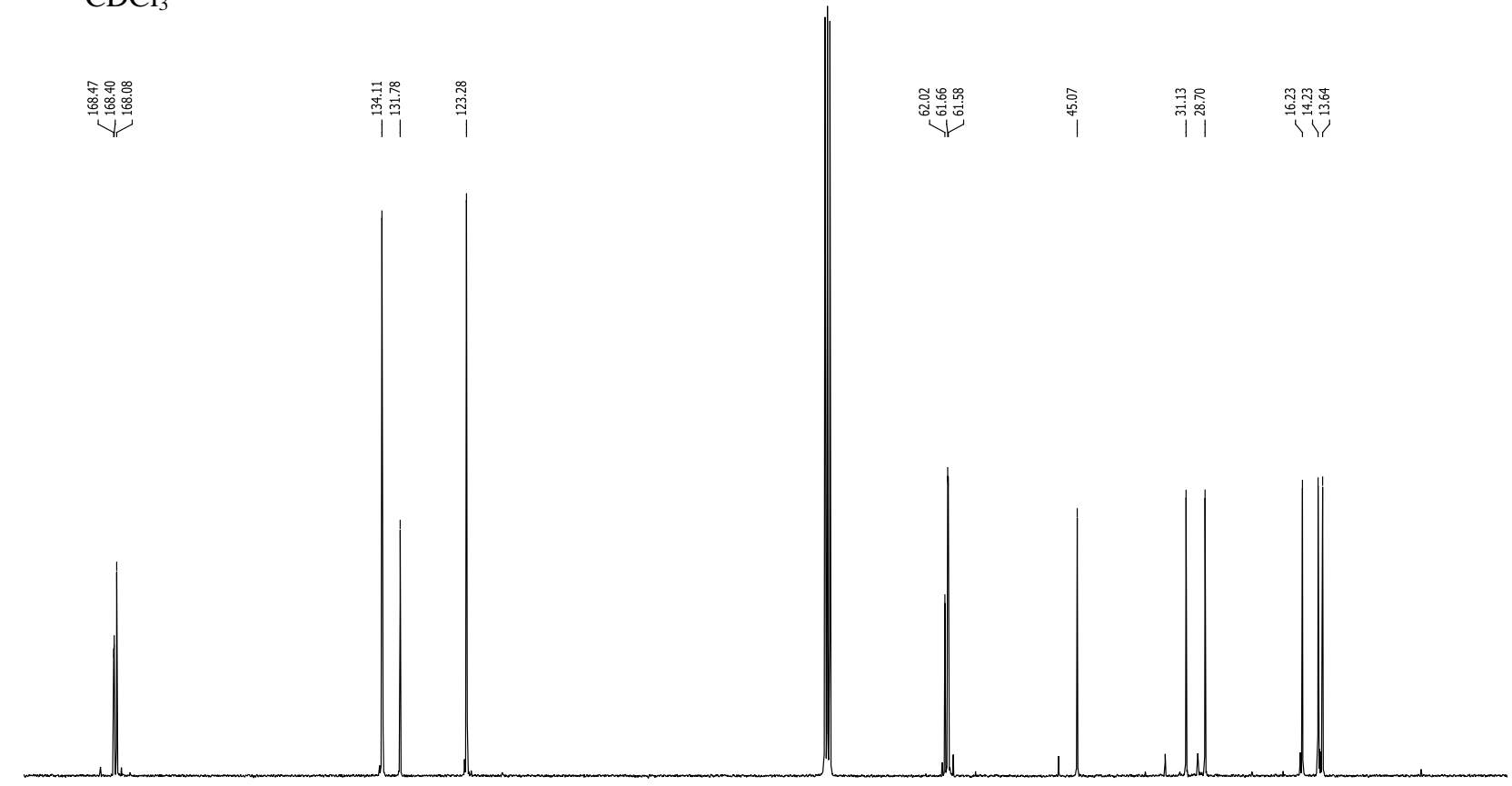

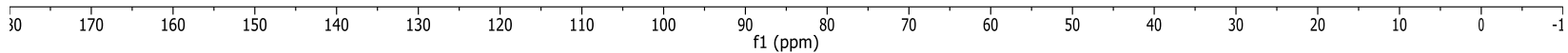



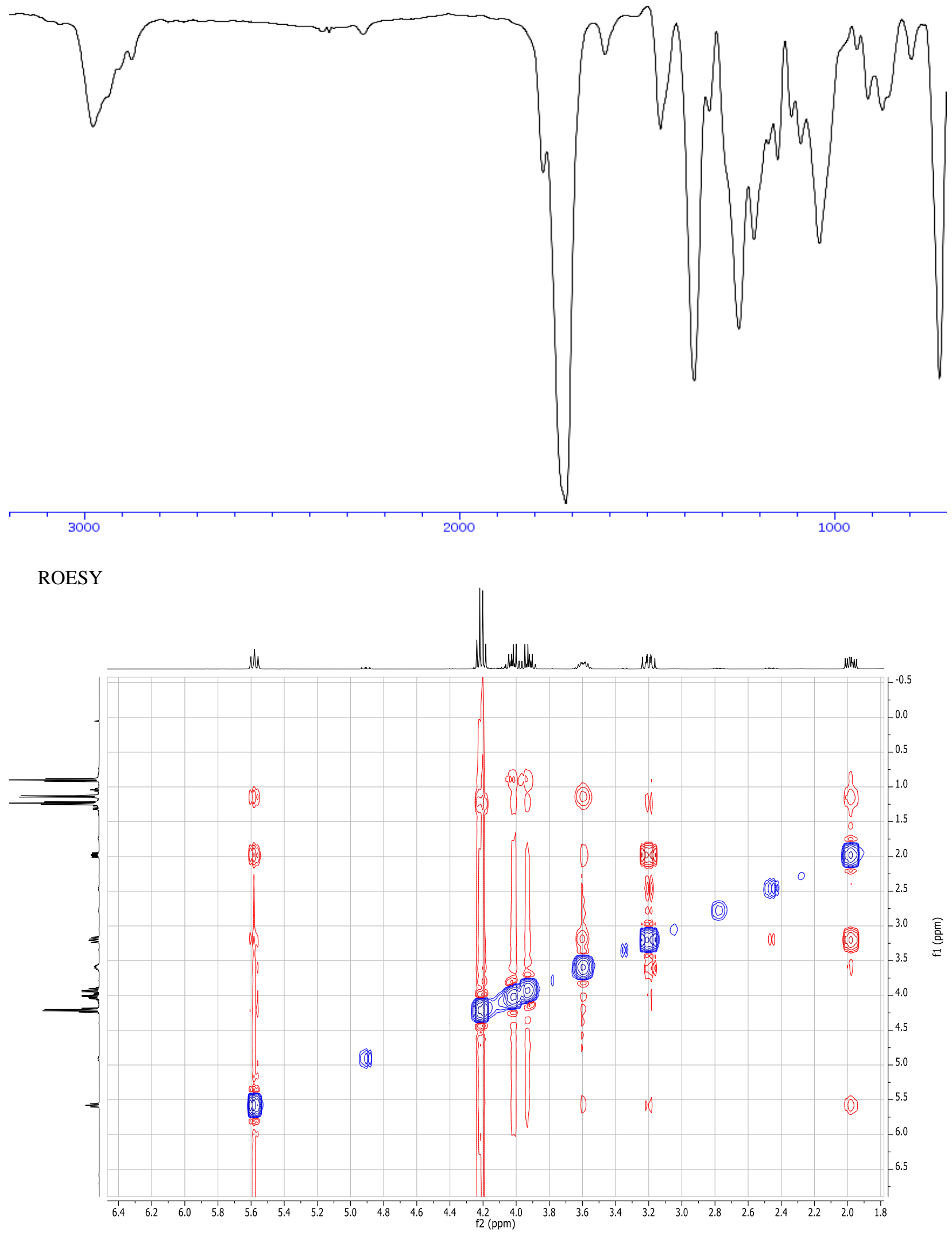

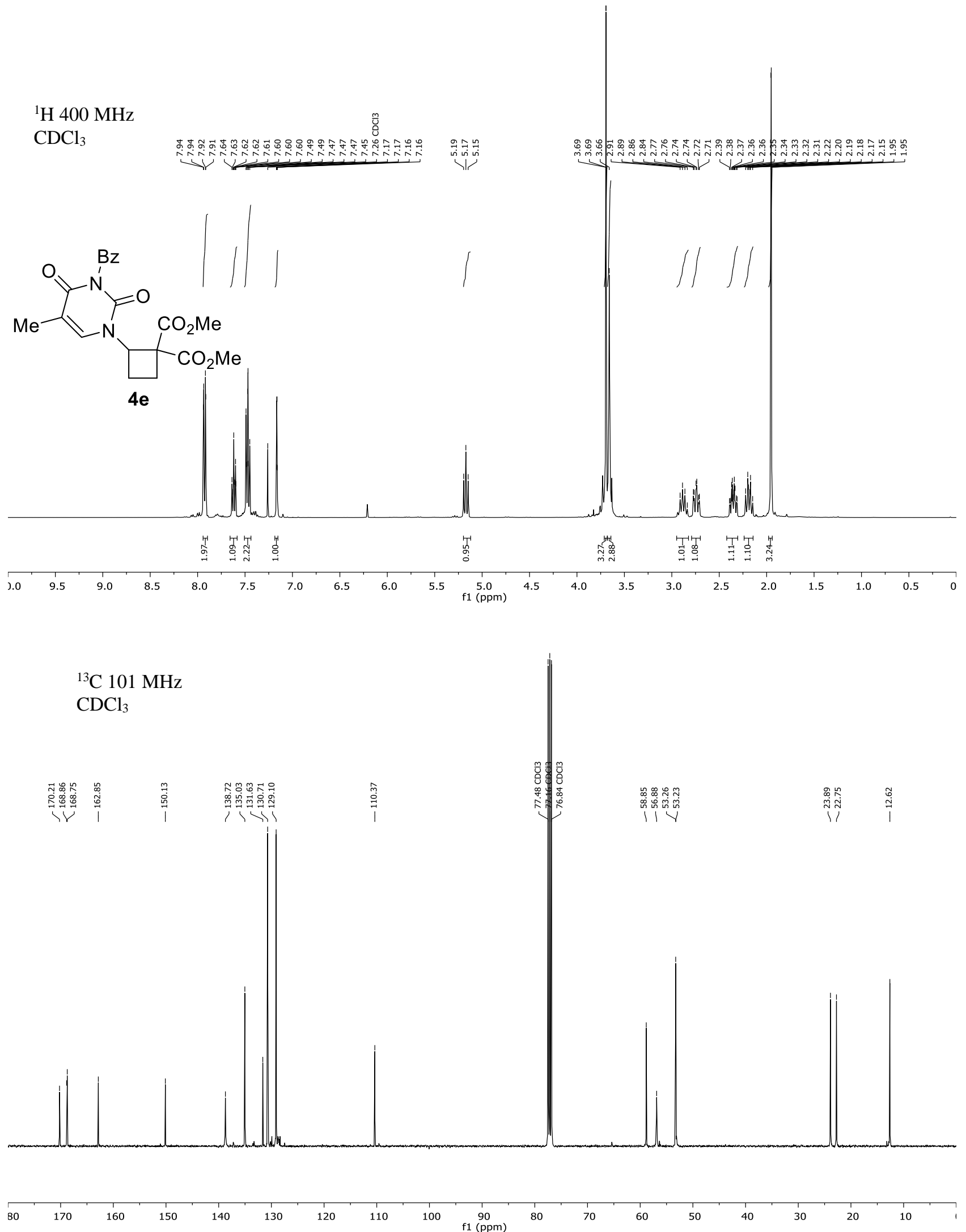


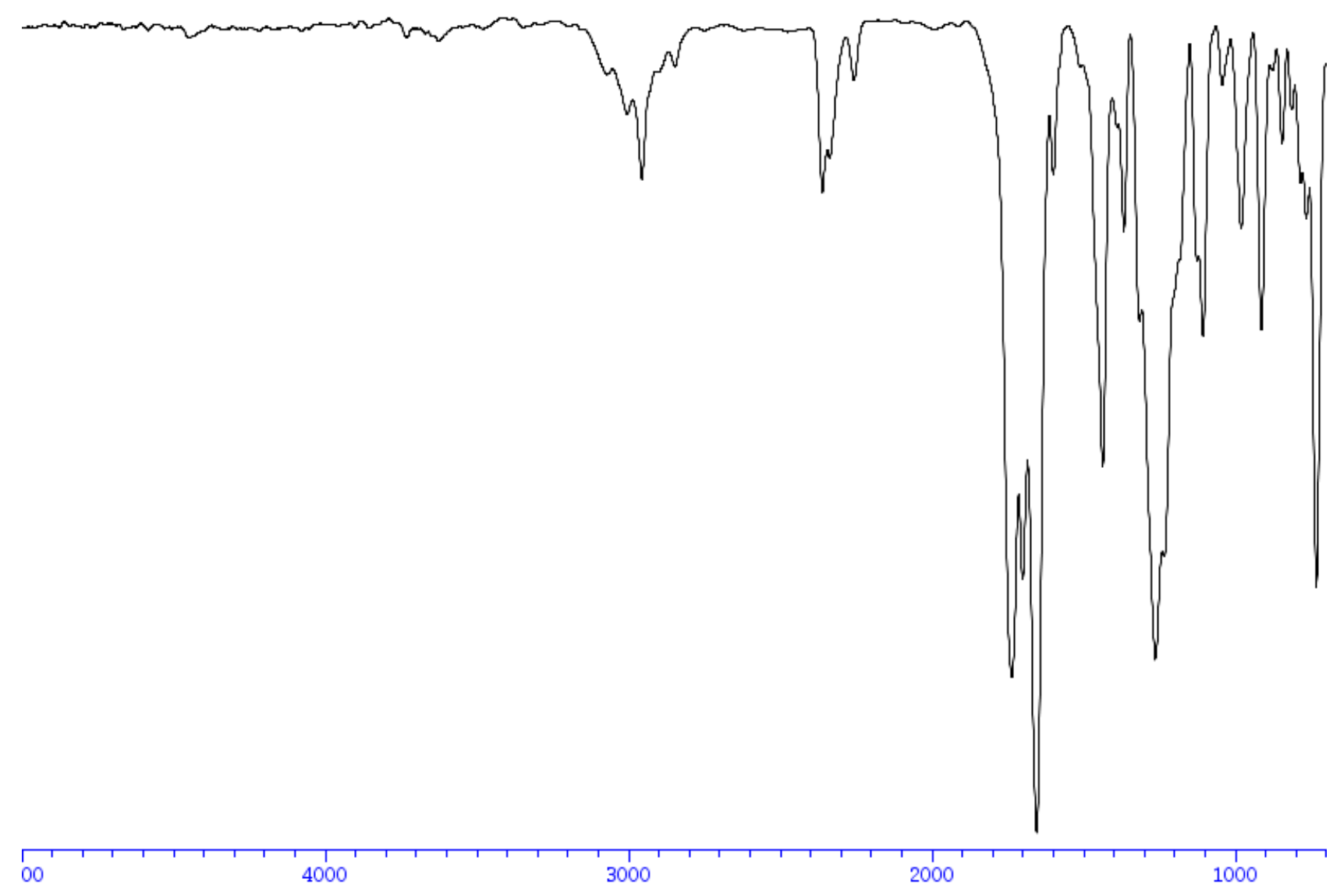




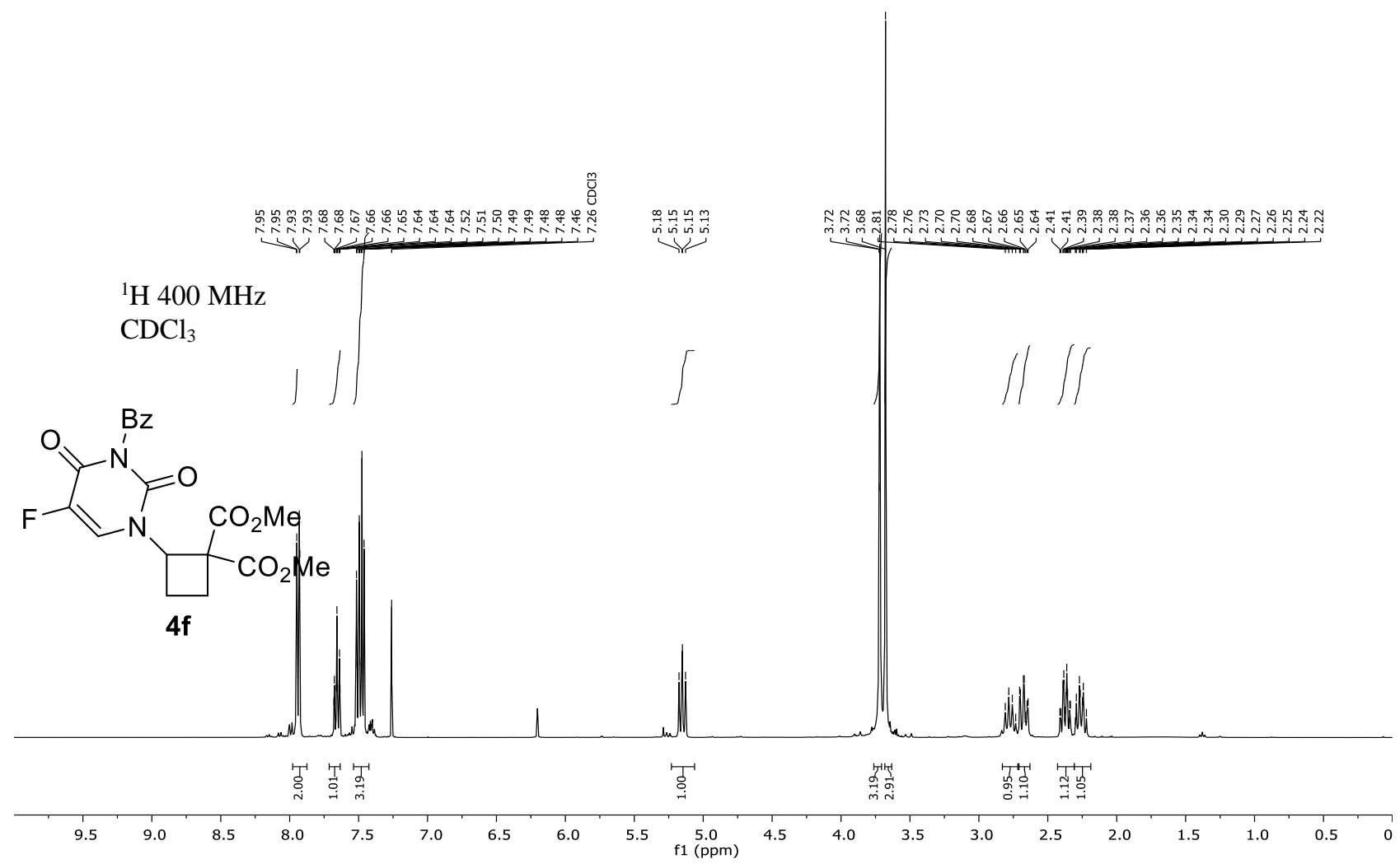

${ }^{13} \mathrm{C} 101 \mathrm{MHz}$

$\mathrm{CDCl}_{3}$

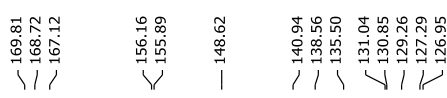

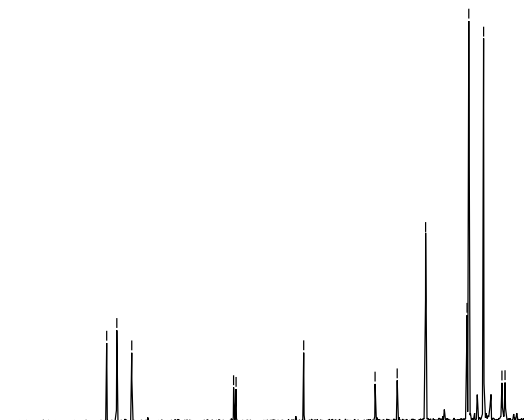

कำ

$11 Y$

㲽
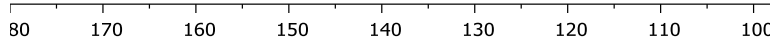

$\stackrel{90}{\mathrm{f} 1} \stackrel{\mathrm{ppm}}{ }$ 


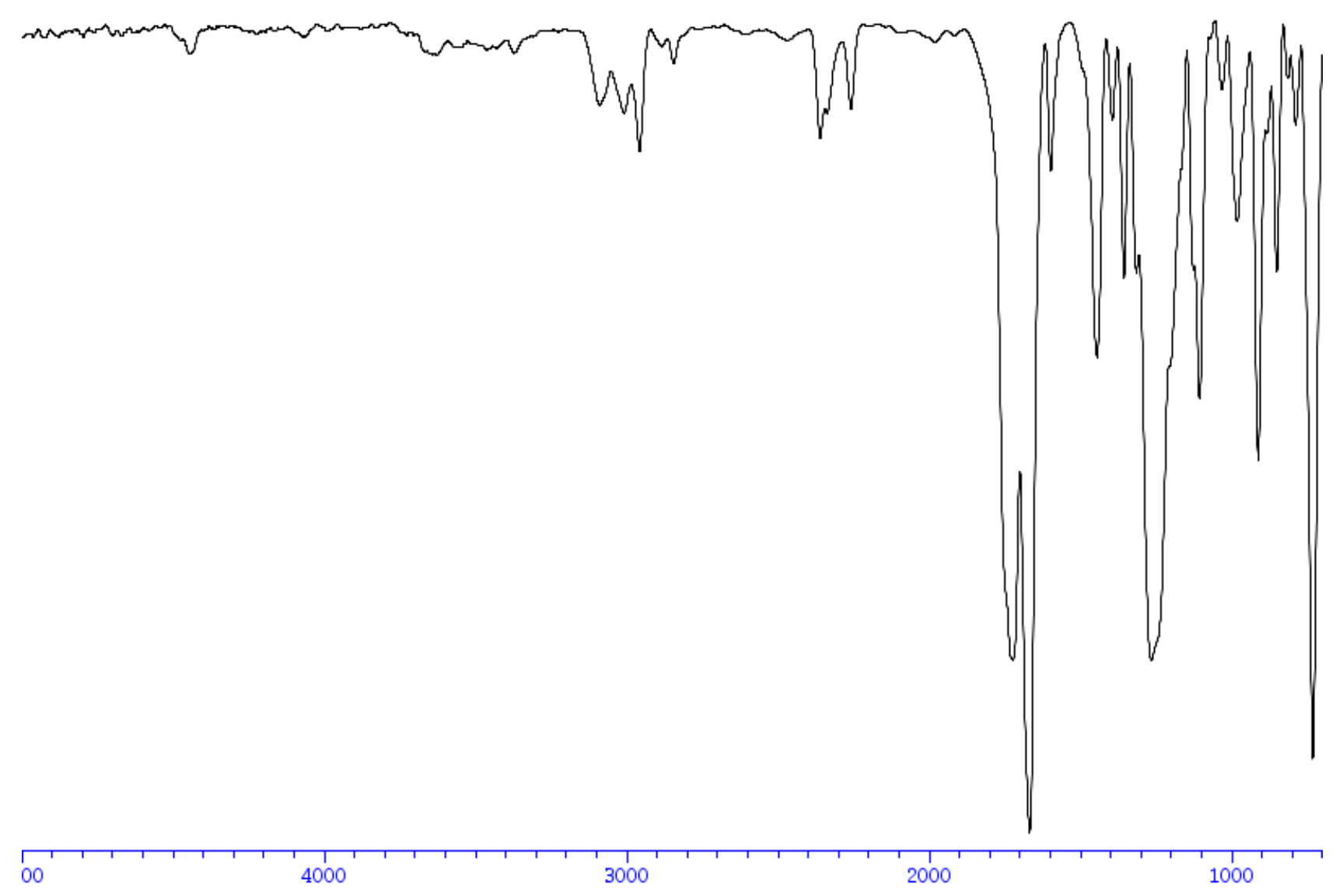




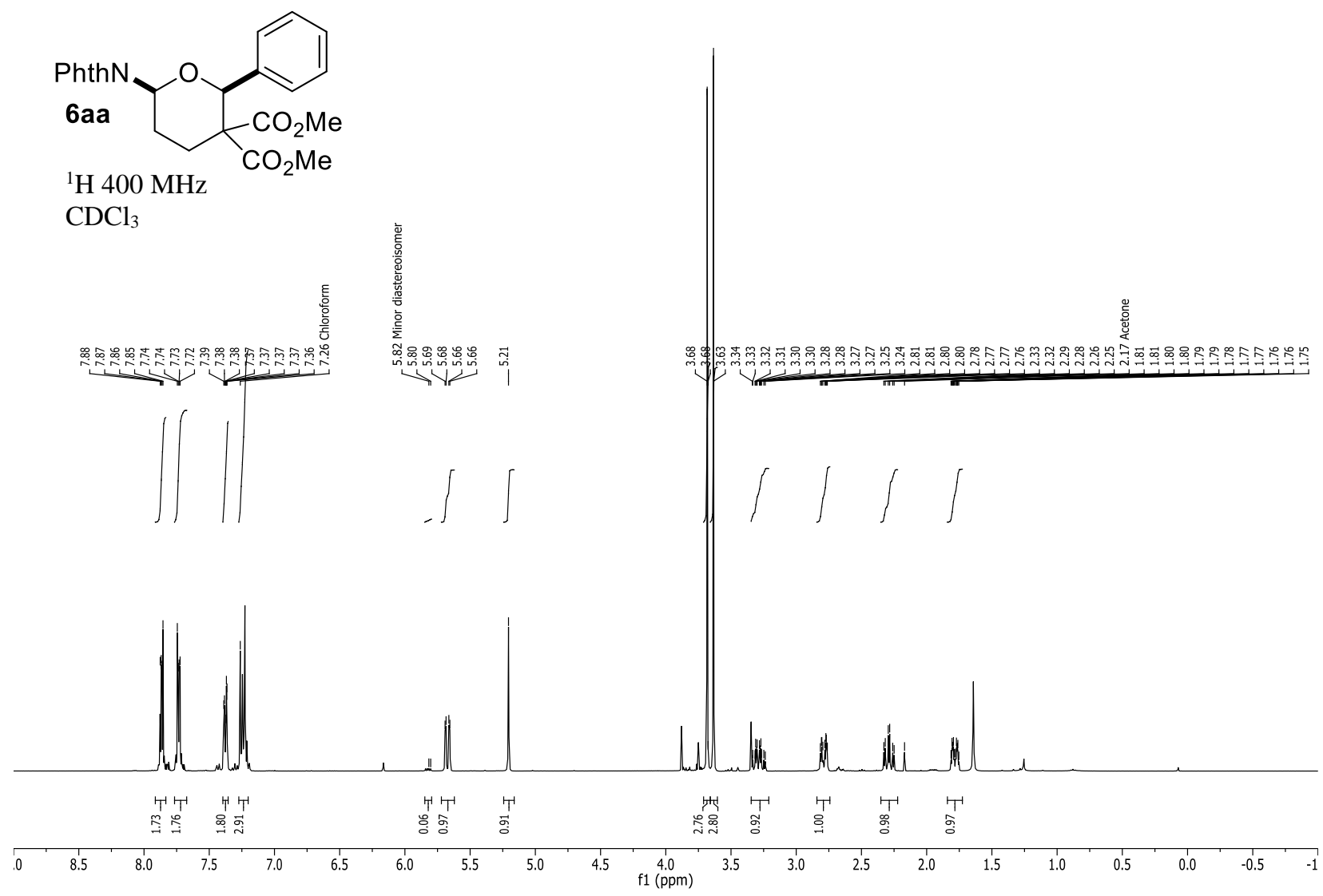

${ }^{13} \mathrm{C} 100 \mathrm{MHz}$ $\mathrm{CDCl}_{3}$
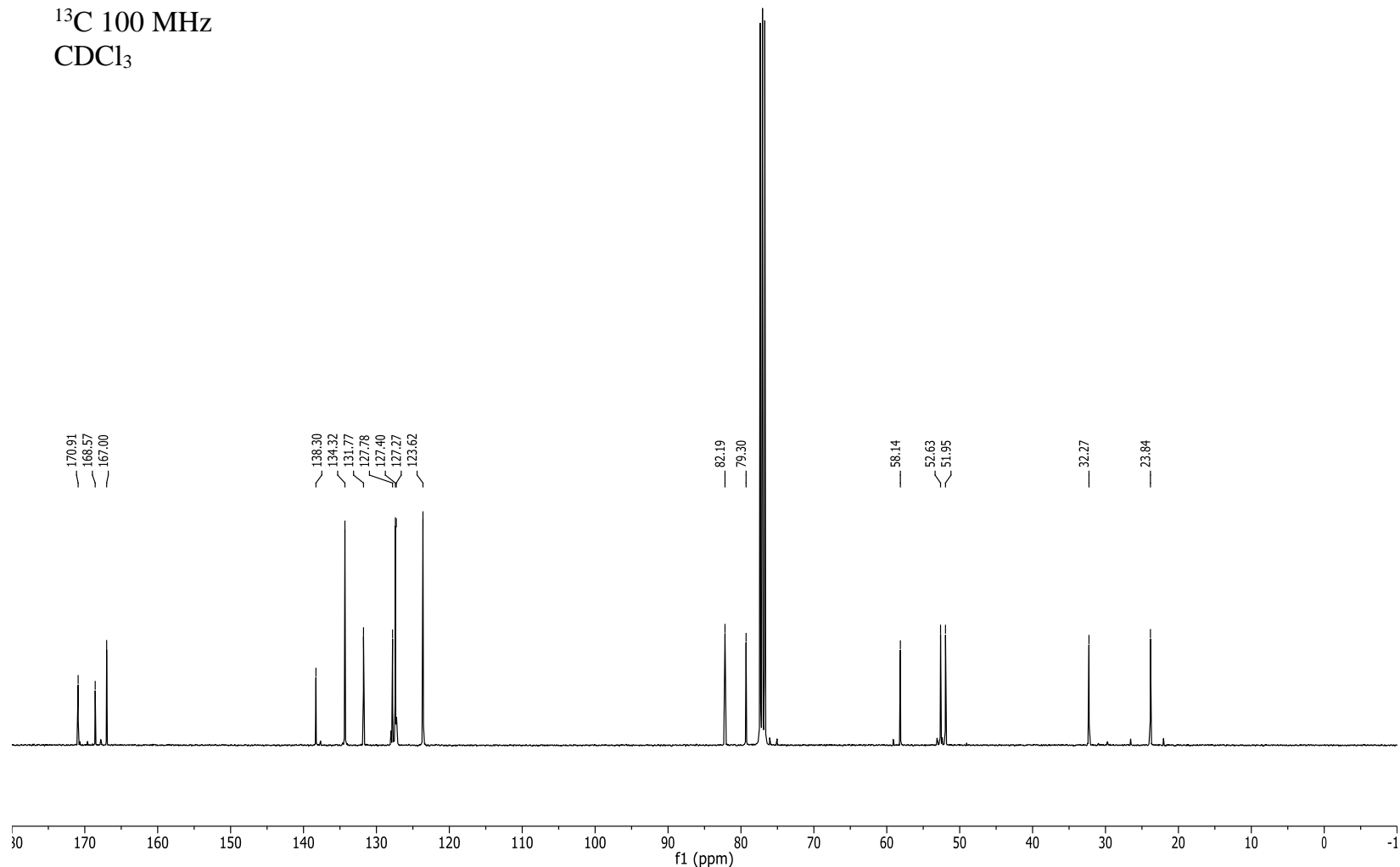

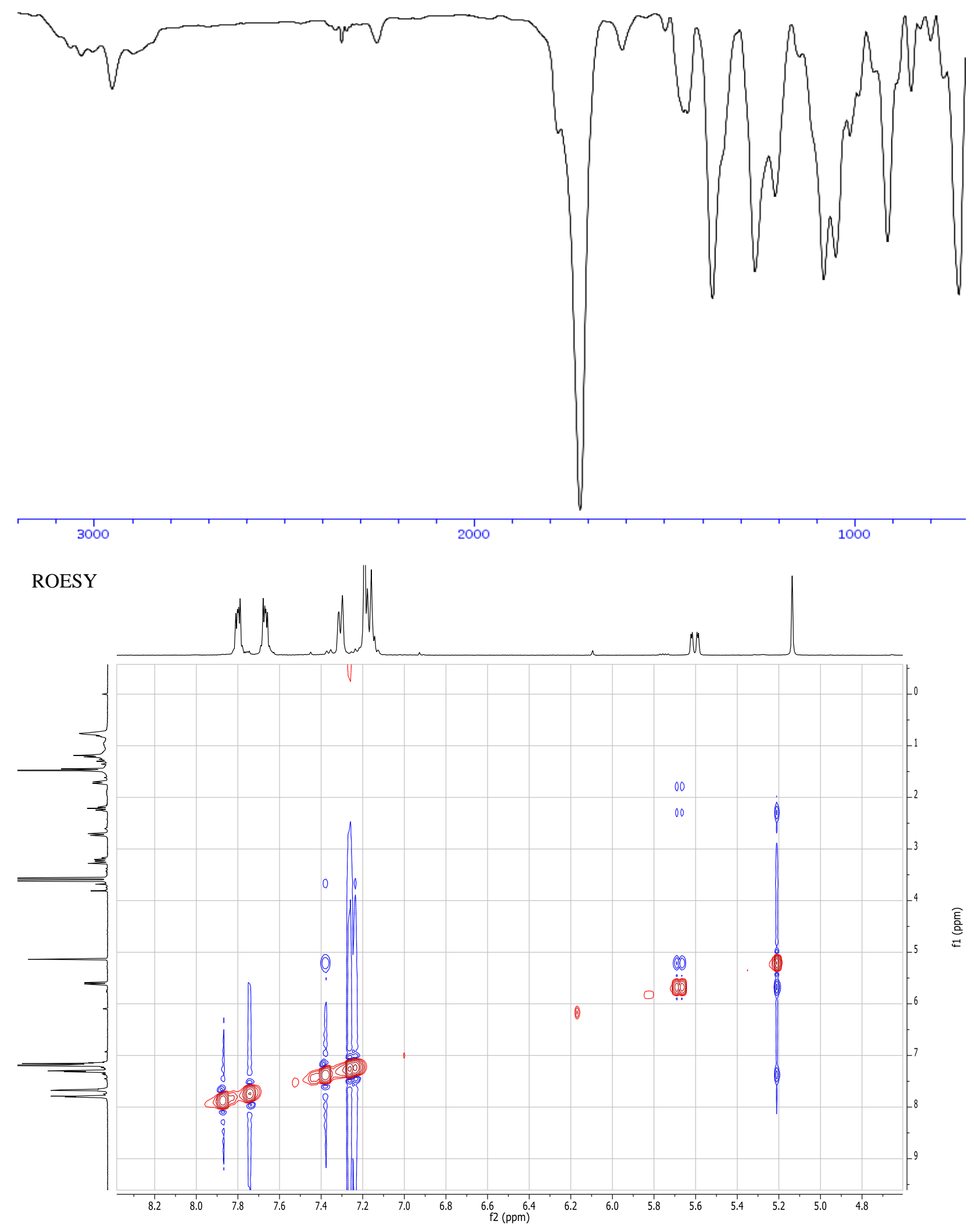


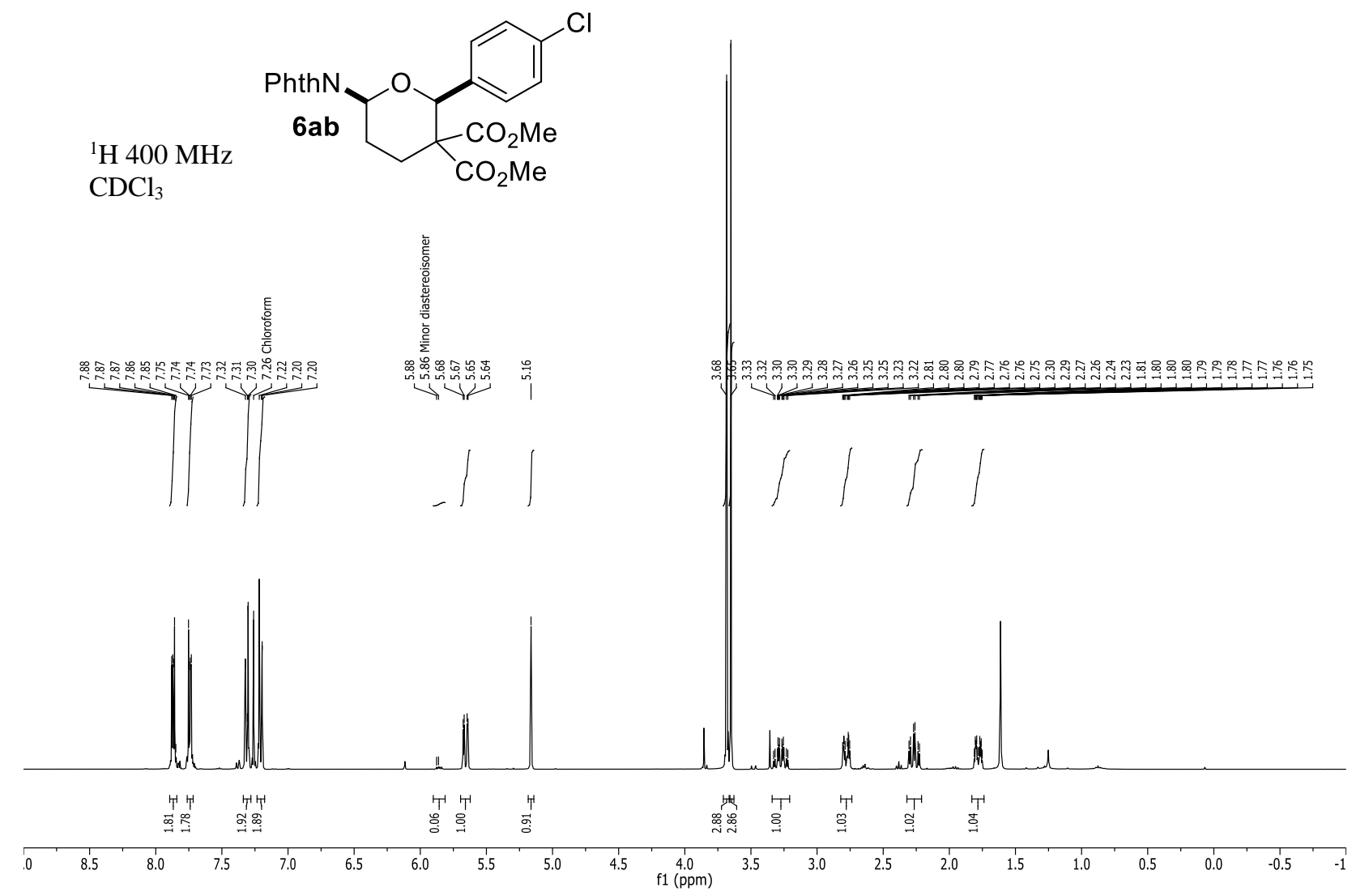

${ }^{13} \mathrm{C} 100 \mathrm{MHz}$

$\mathrm{CDCl}_{3}$

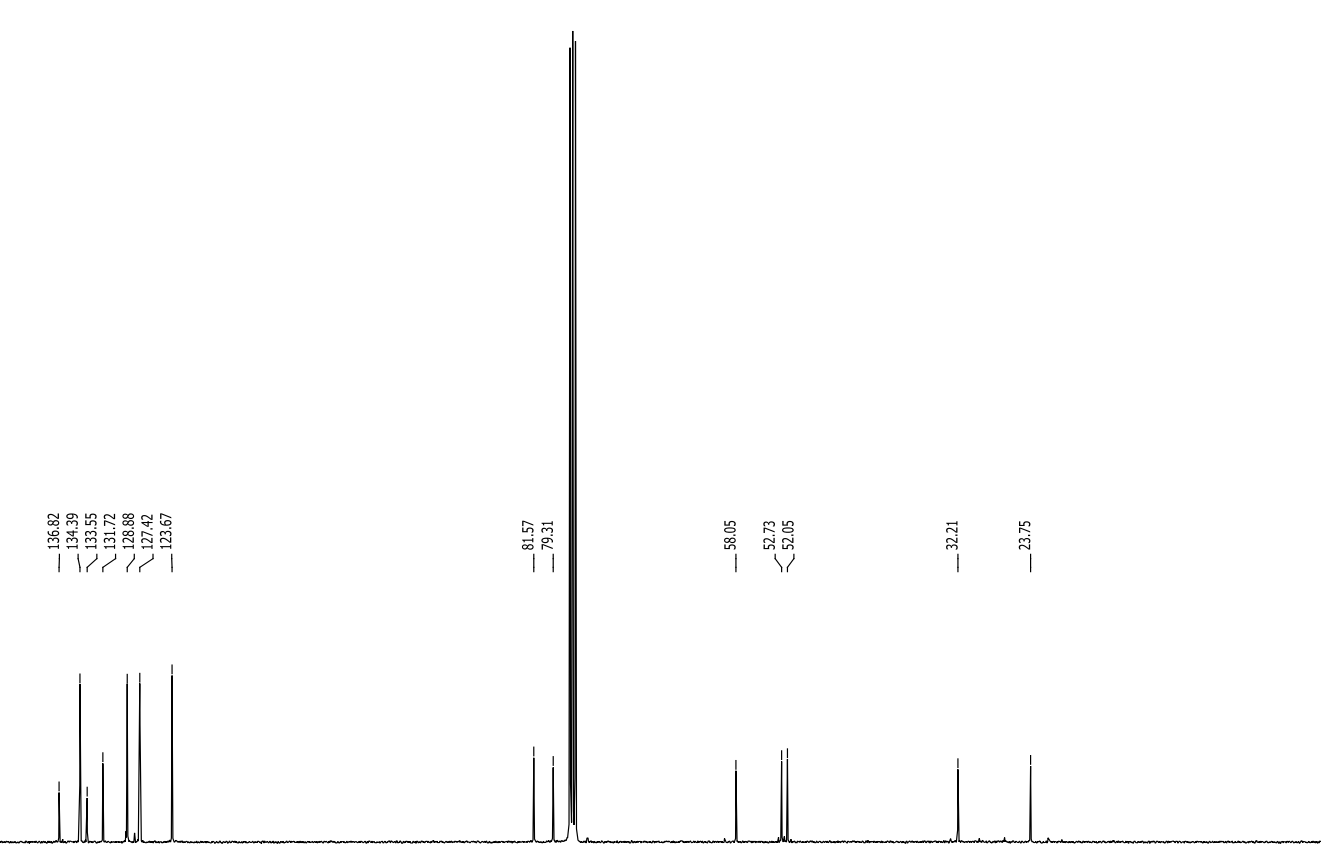

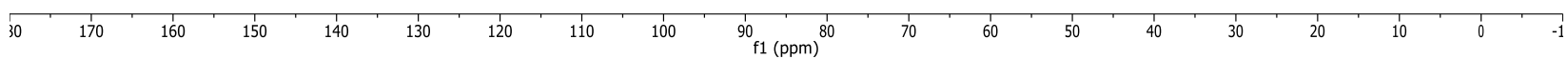




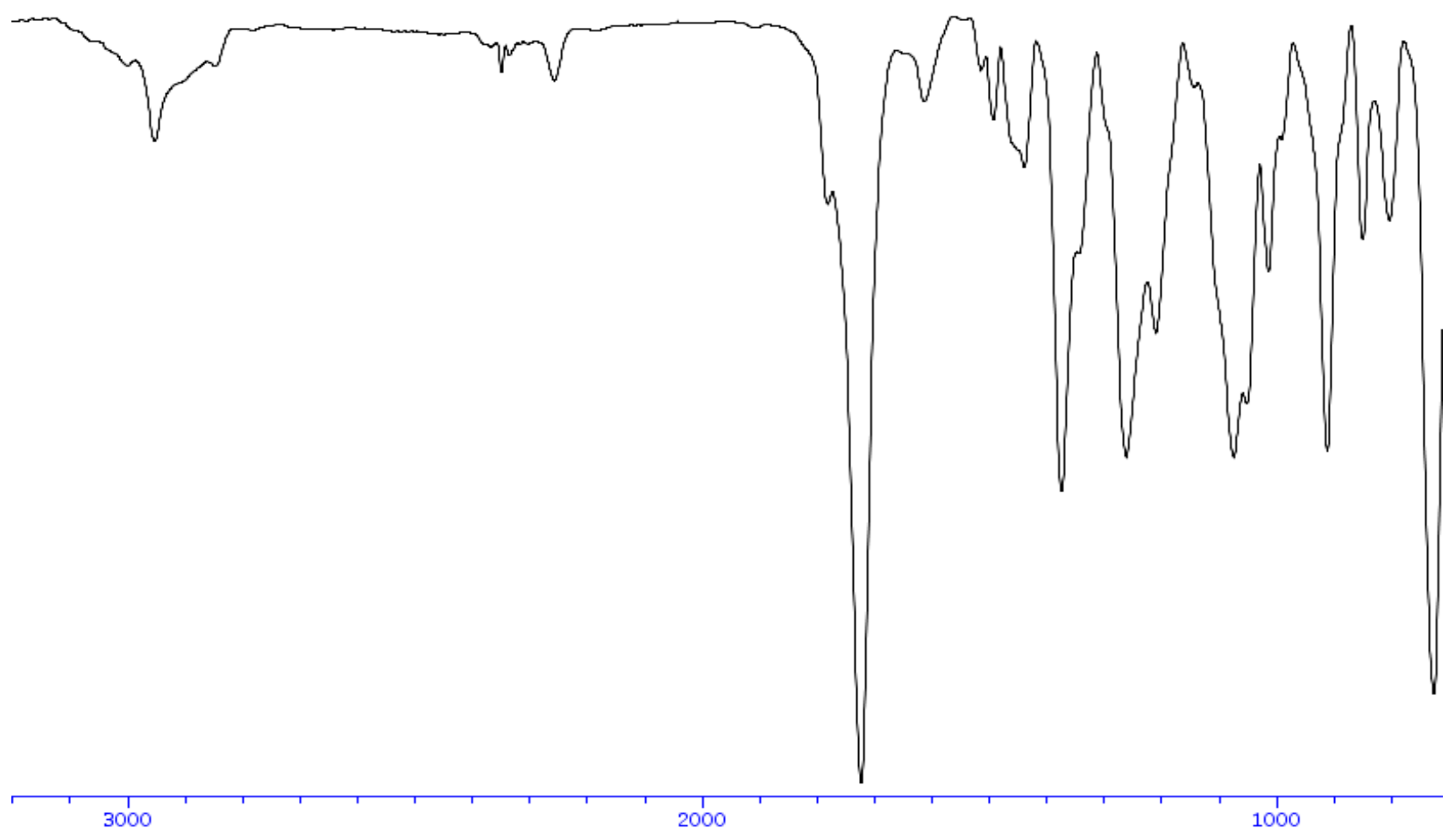




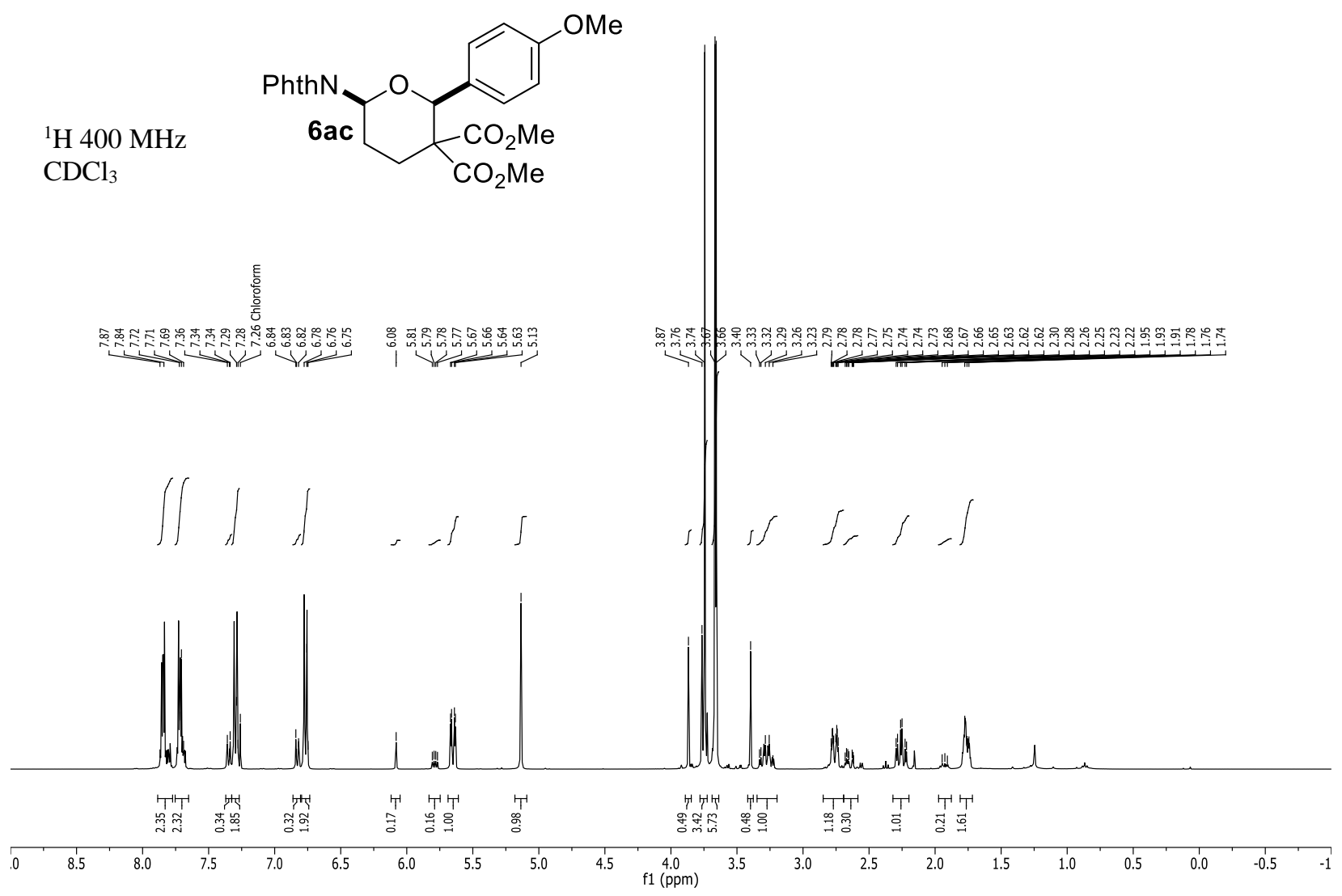

${ }^{13} \mathrm{C} 100 \mathrm{MHz}$ $\mathrm{CDCl}_{3}$

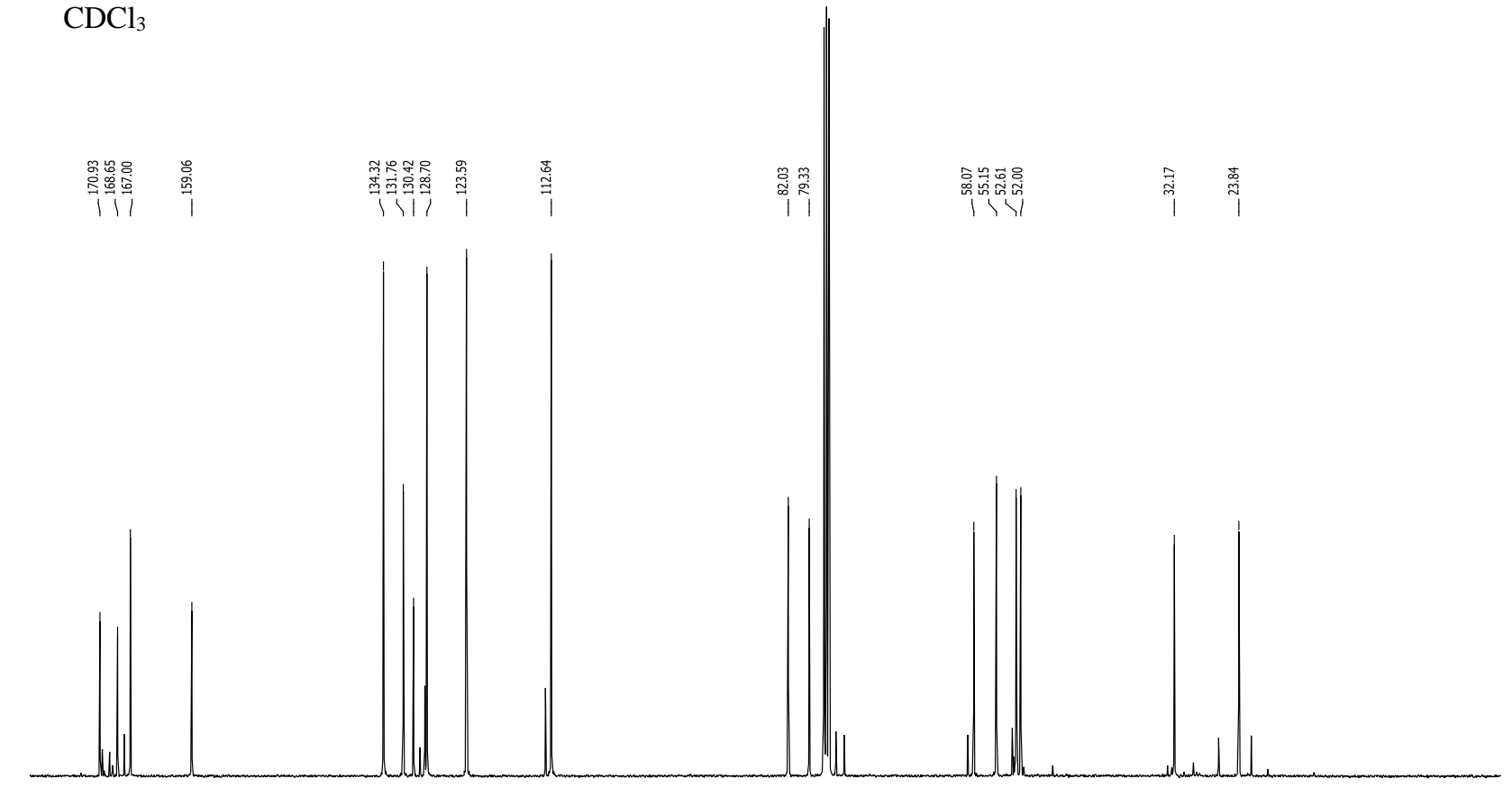

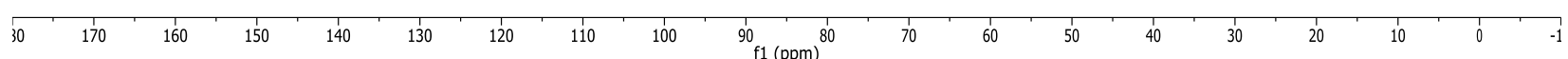




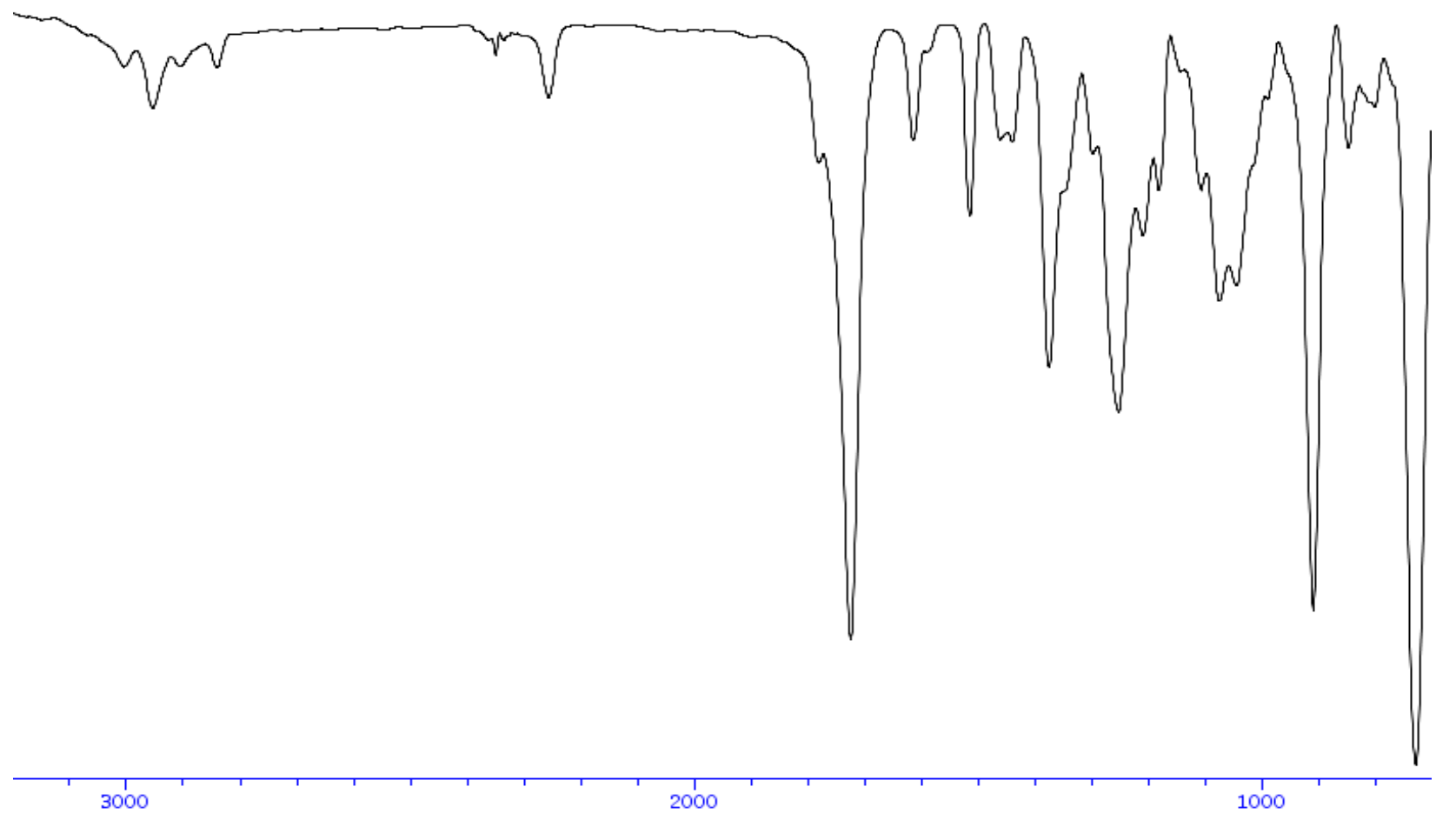




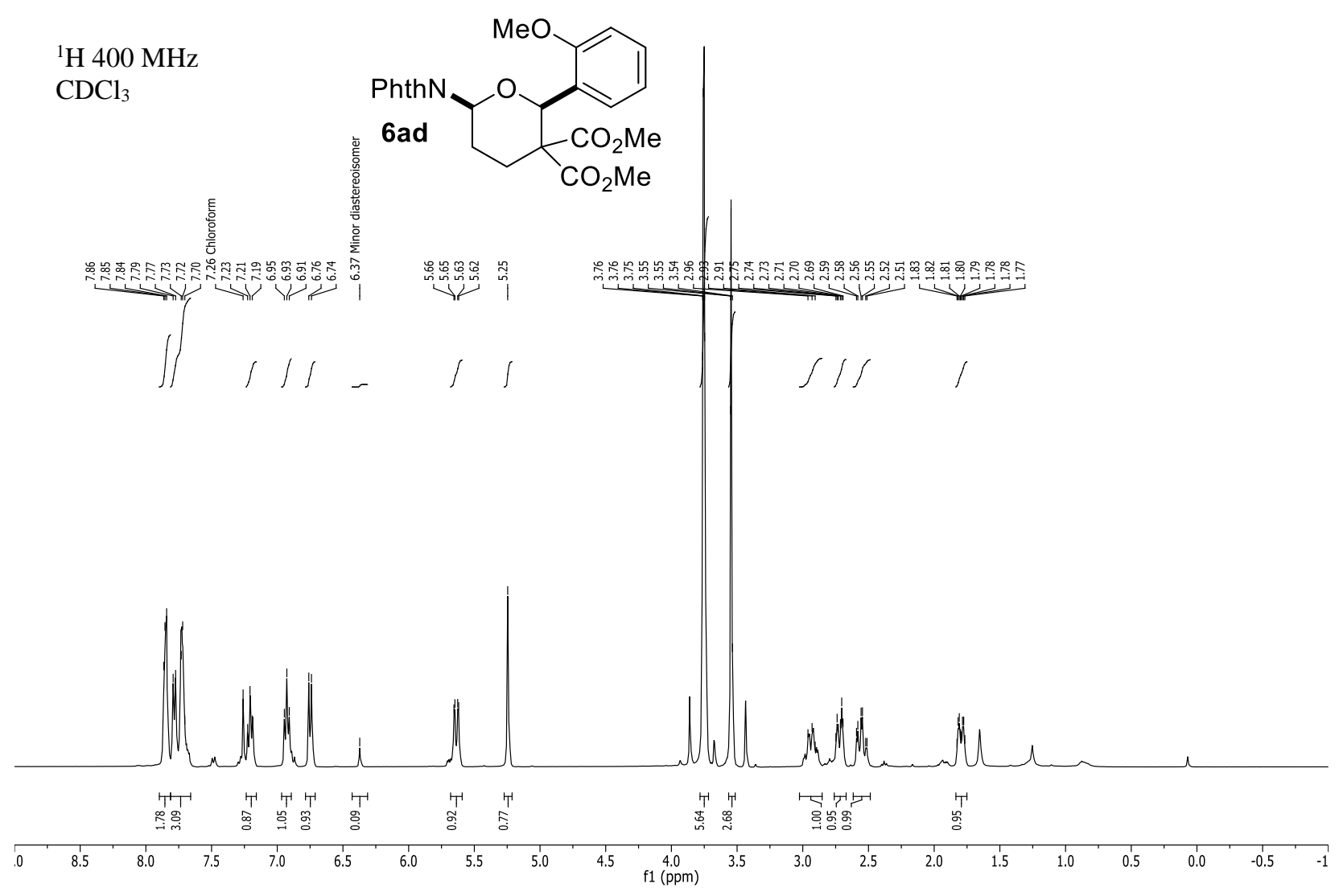

${ }^{13} \mathrm{C} 100 \mathrm{MHz}$

$\mathrm{CDCl}_{3}$

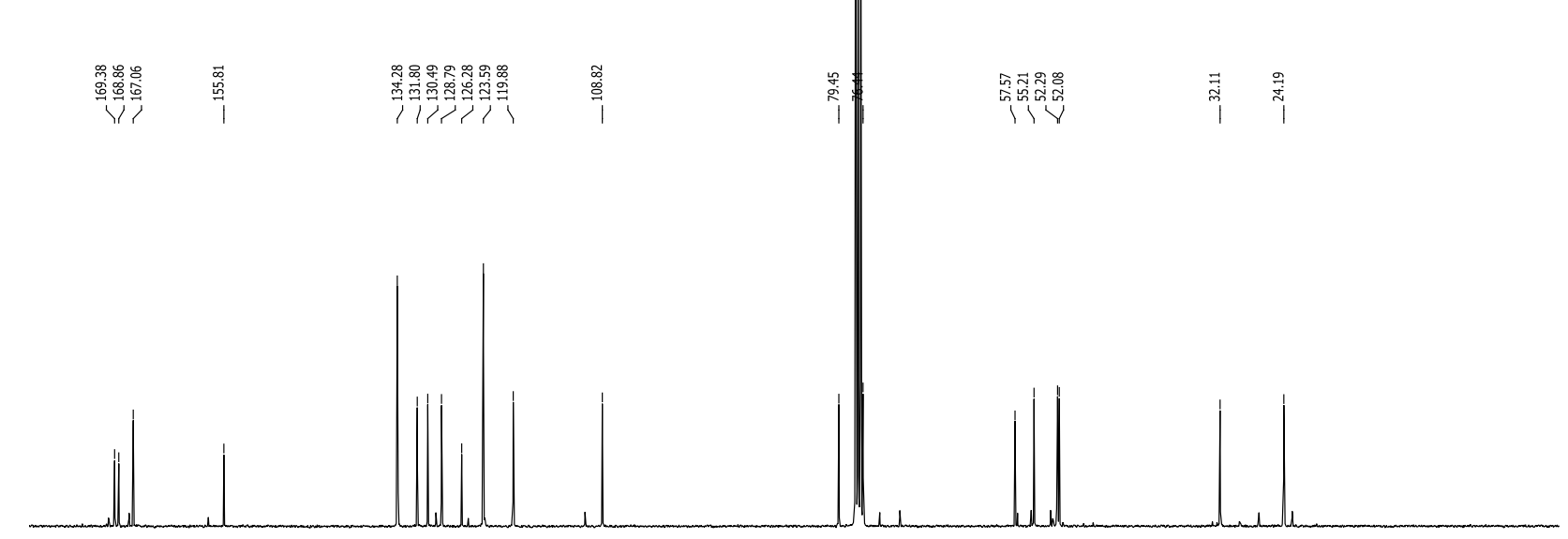

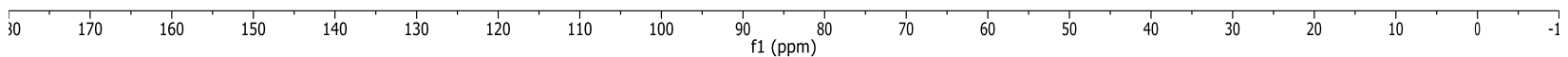




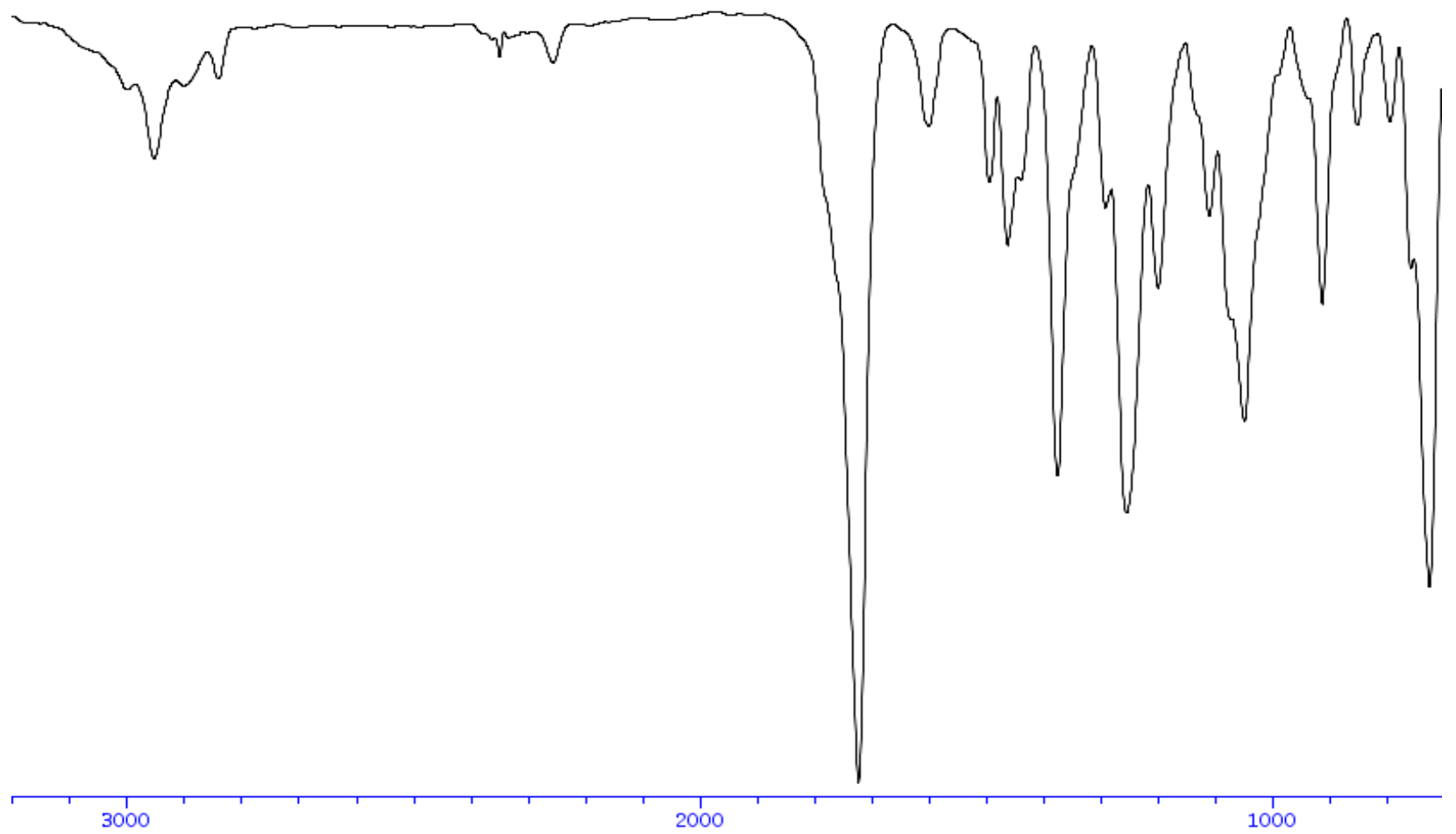




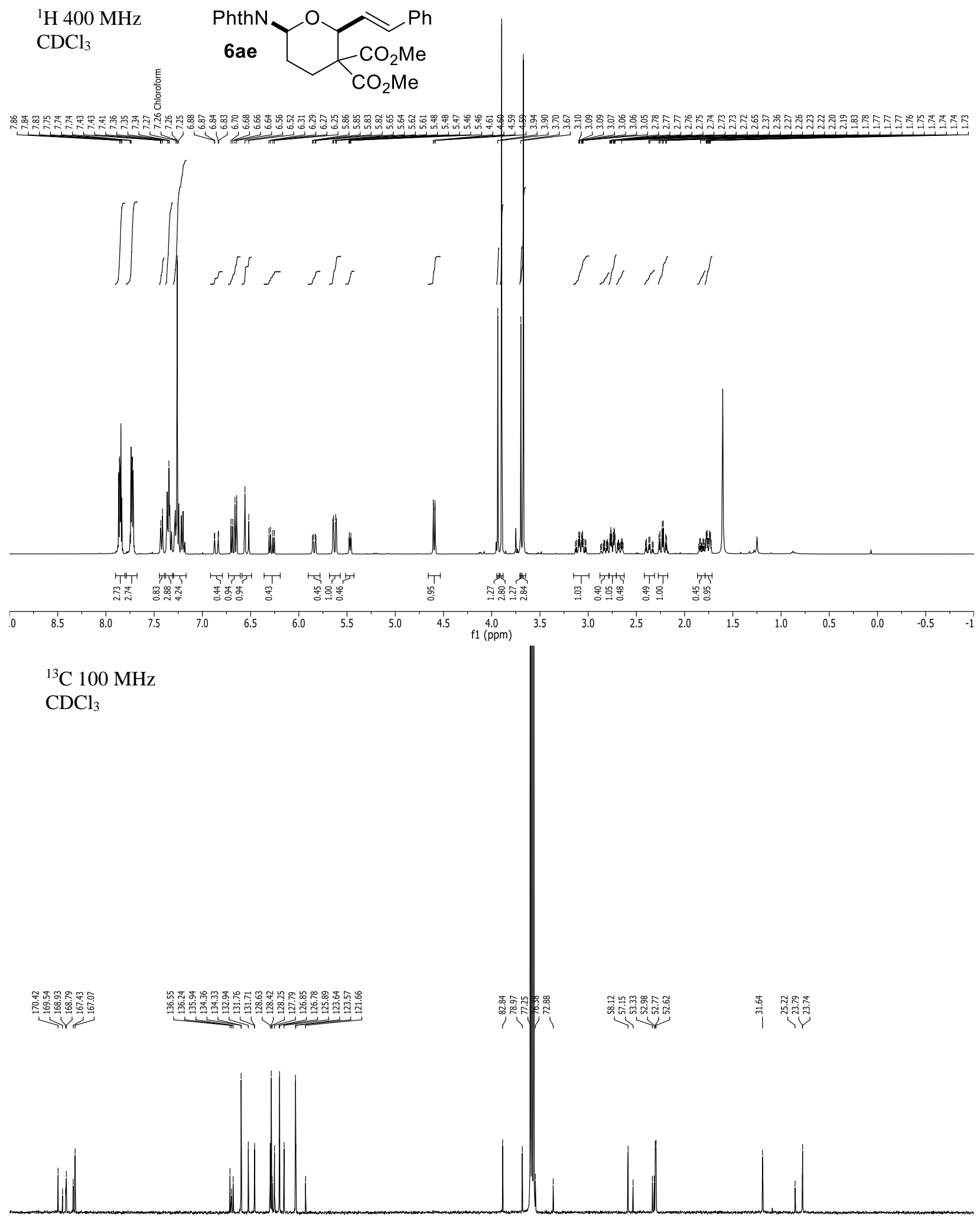

\begin{tabular}{llllllllll}
\hline 1 & 170 & 160 & 150 & 140 & 130 & 120 & 110 & 100 & 90 \\
$\mathrm{f}_{1}(\mathrm{ppm})$ & ${ }^{1}{ }^{1}$
\end{tabular} 


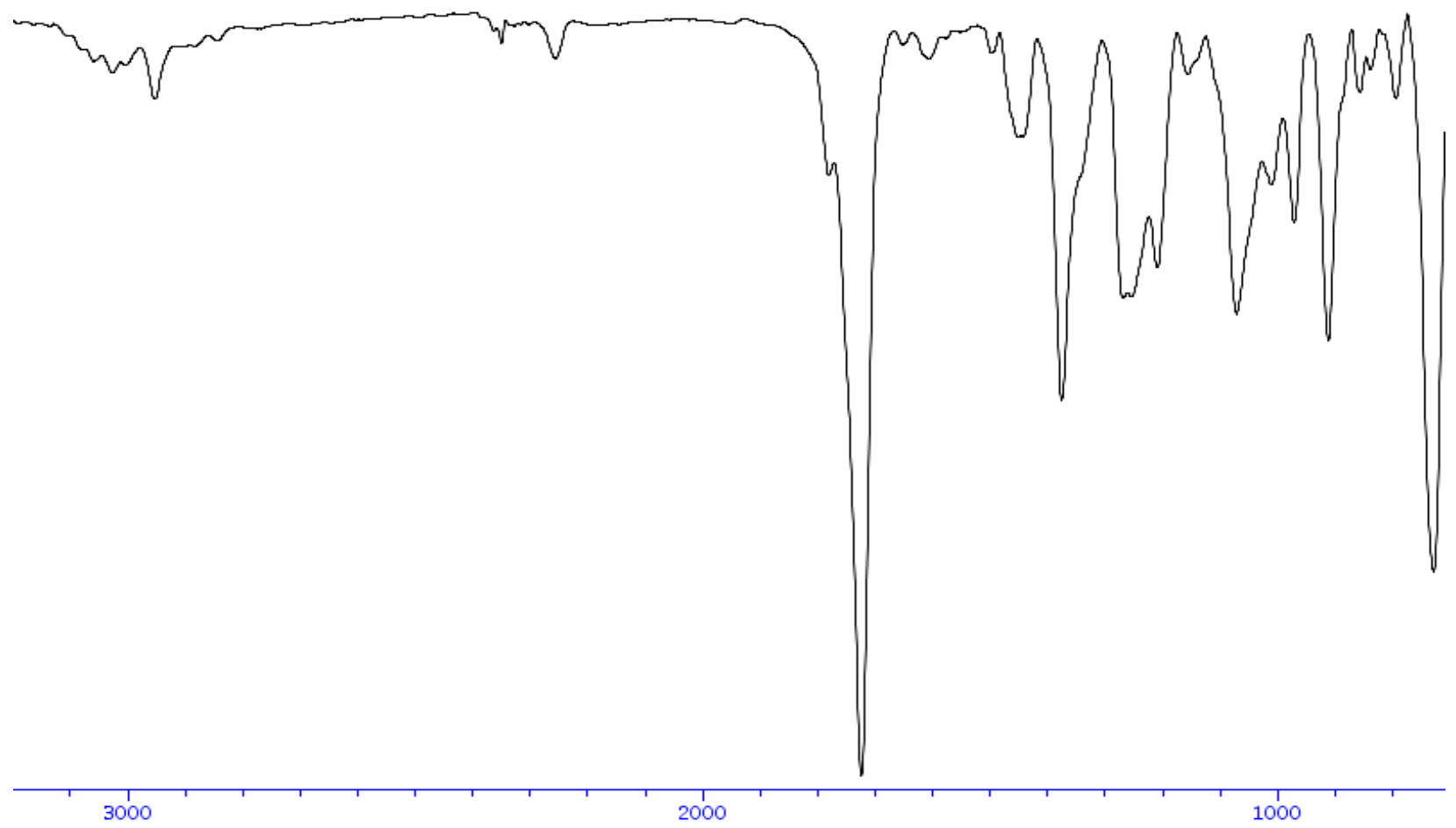




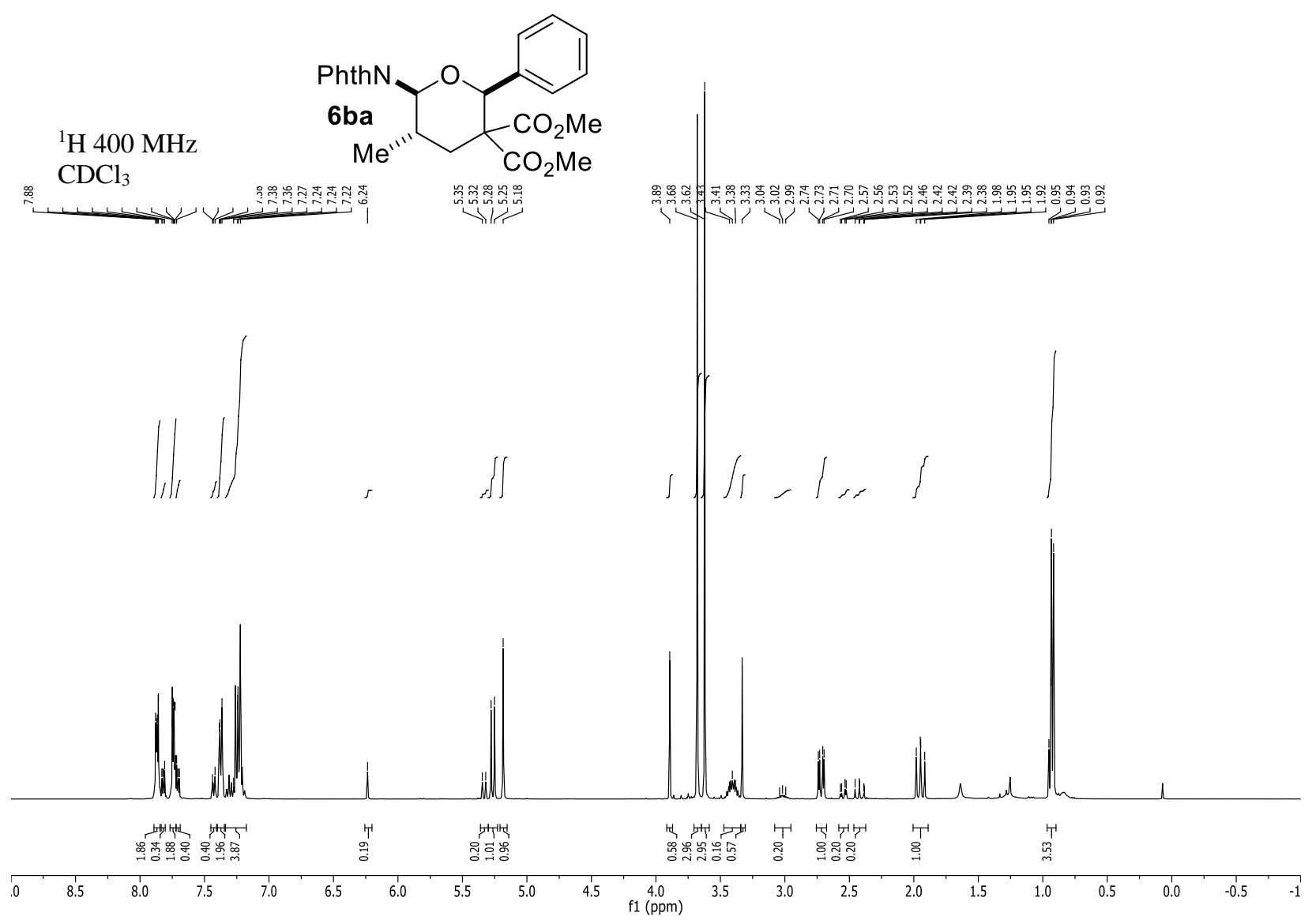

${ }^{13} \mathrm{C} 100 \mathrm{MHz}$ $\mathrm{CDCl}_{3}$

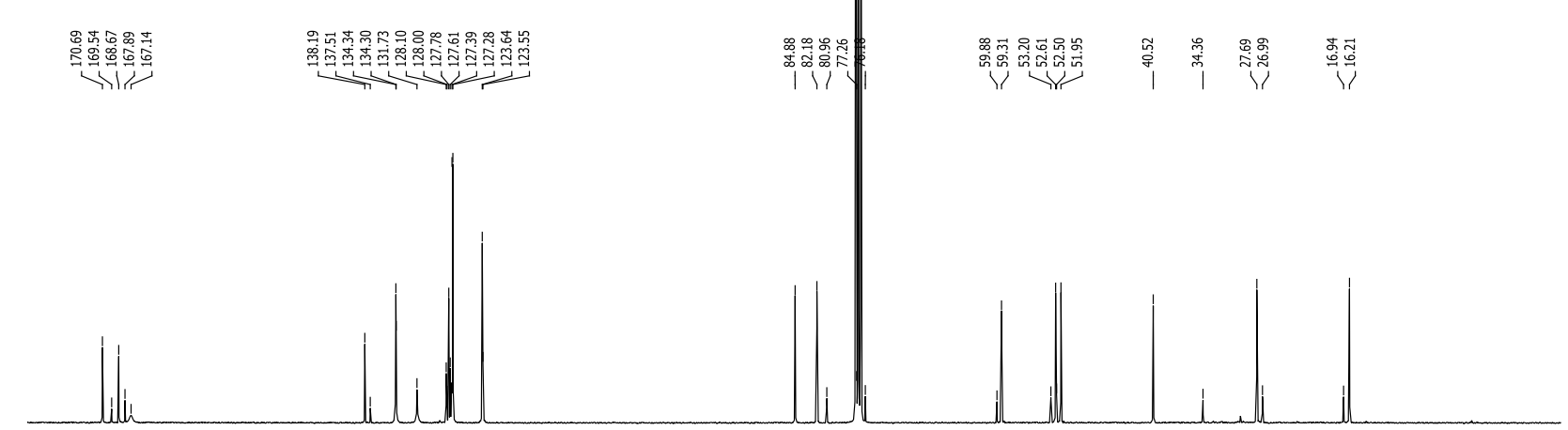

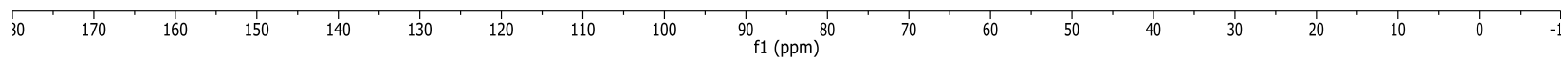




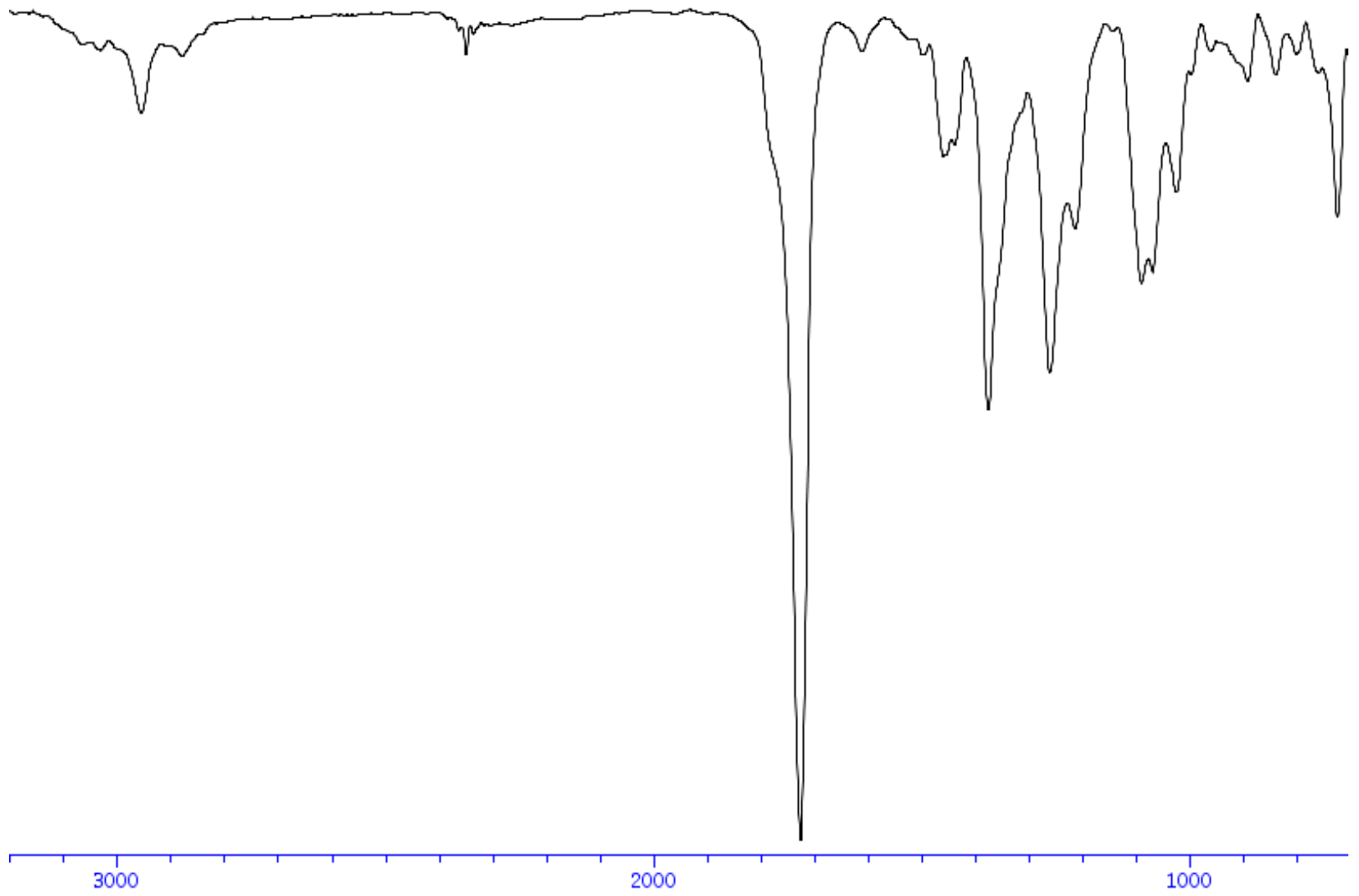




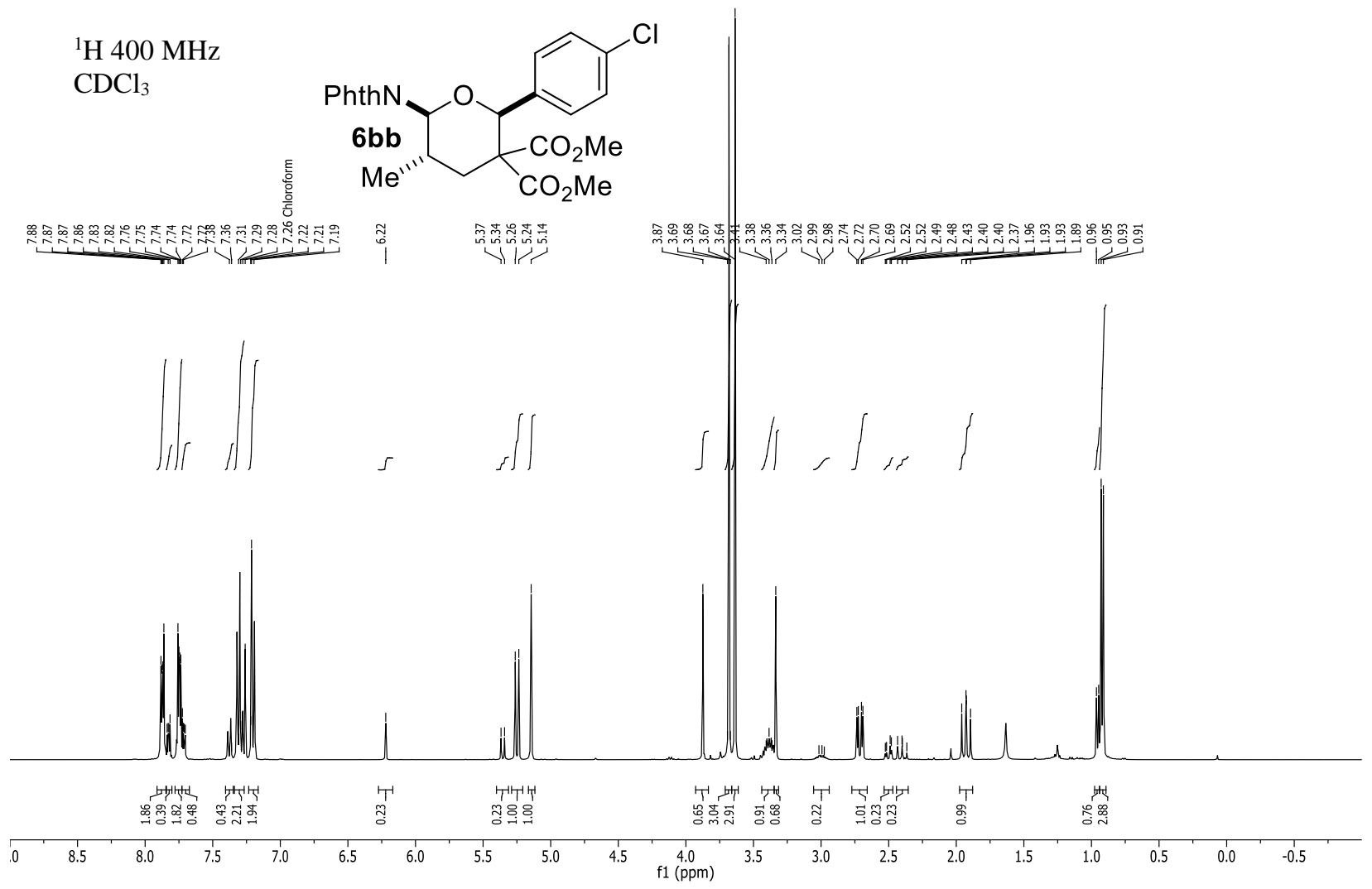

${ }^{13} \mathrm{C} 100 \mathrm{MHz}$ $\mathrm{CDCl}_{3}$

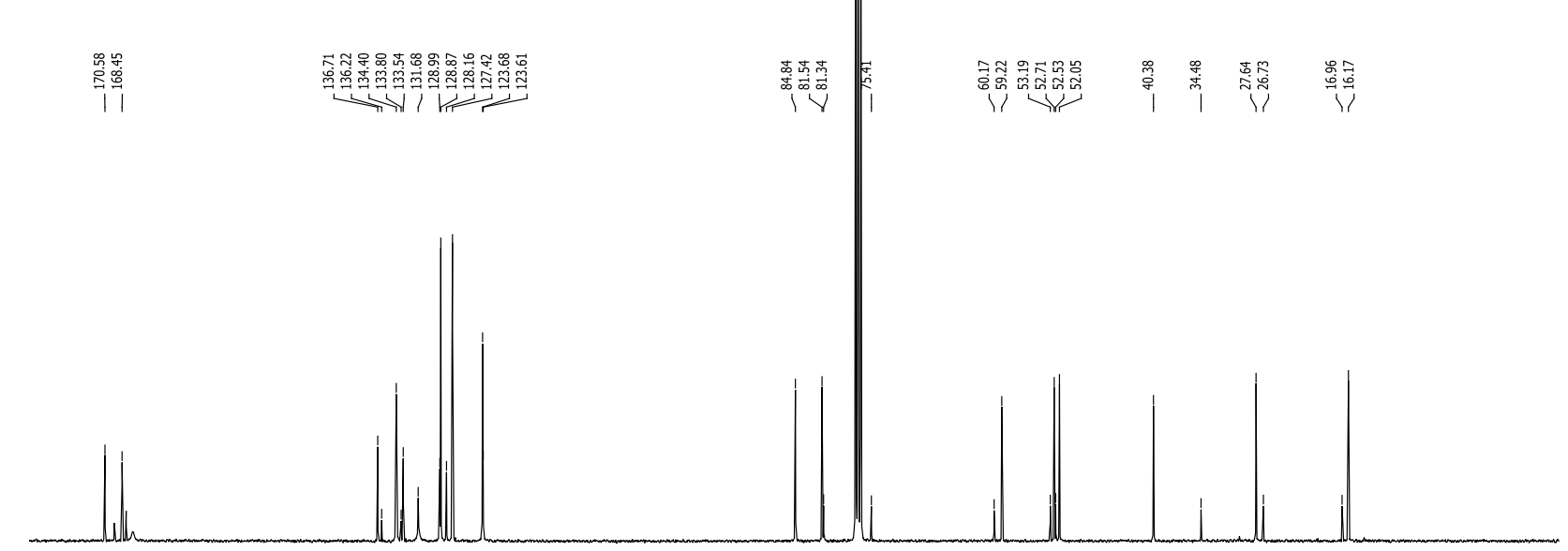

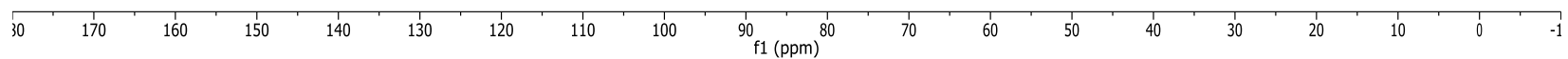




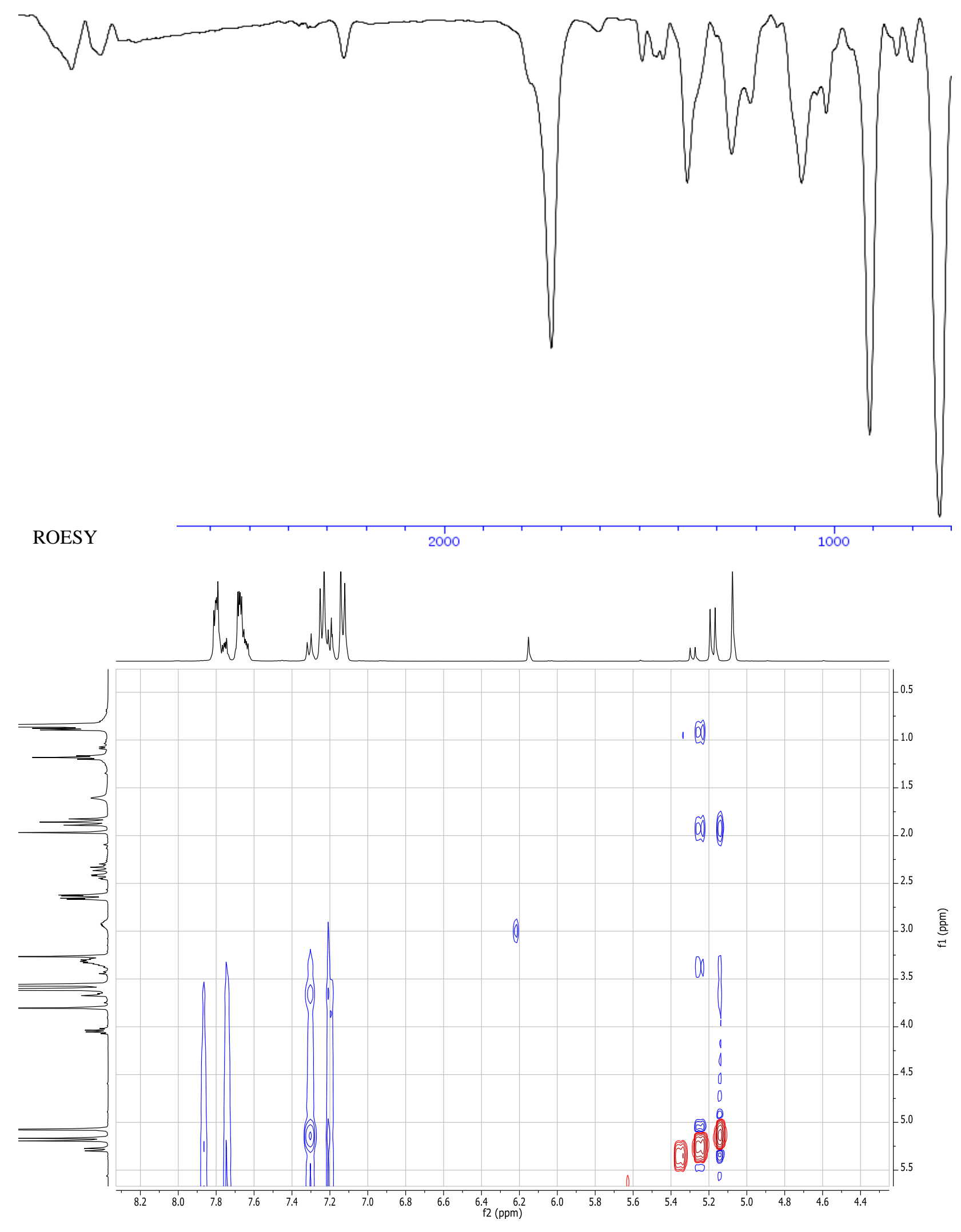




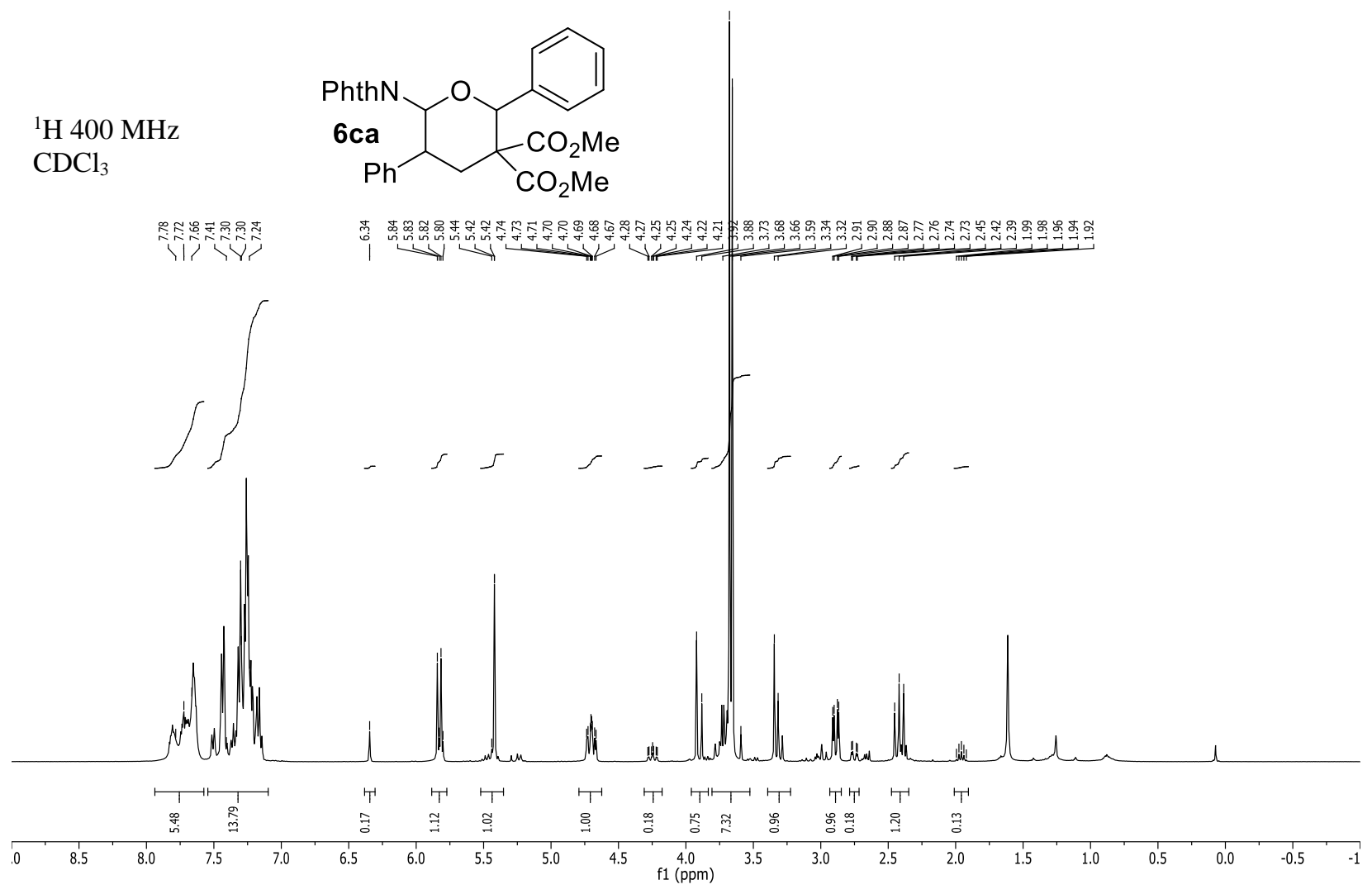

${ }^{13} \mathrm{C} 100 \mathrm{MHz}$

$\mathrm{CDCl}_{3}$

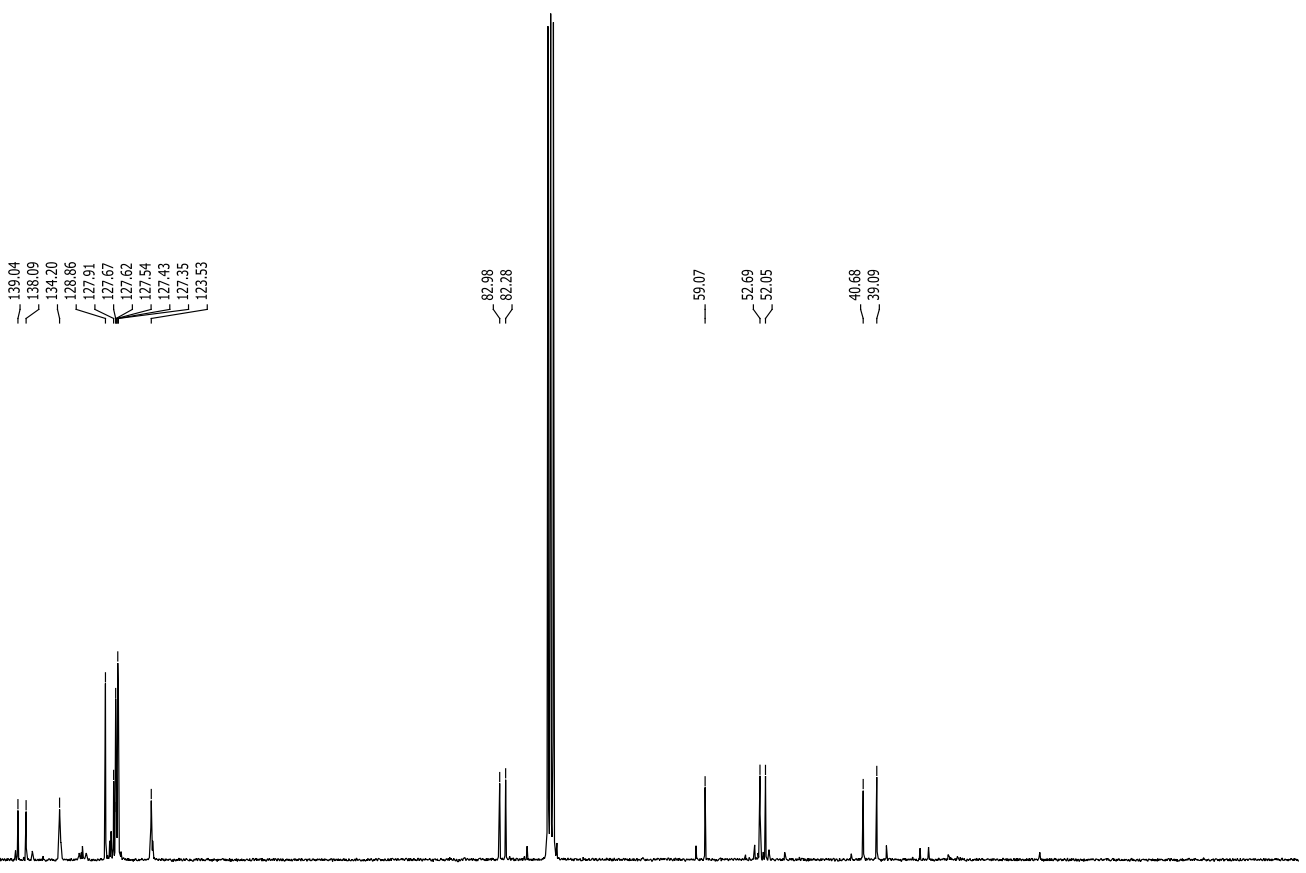

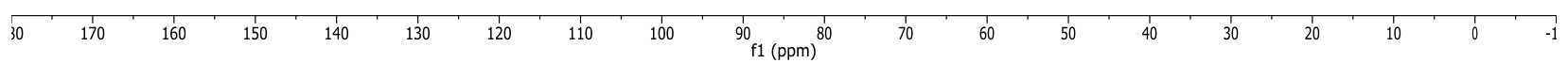




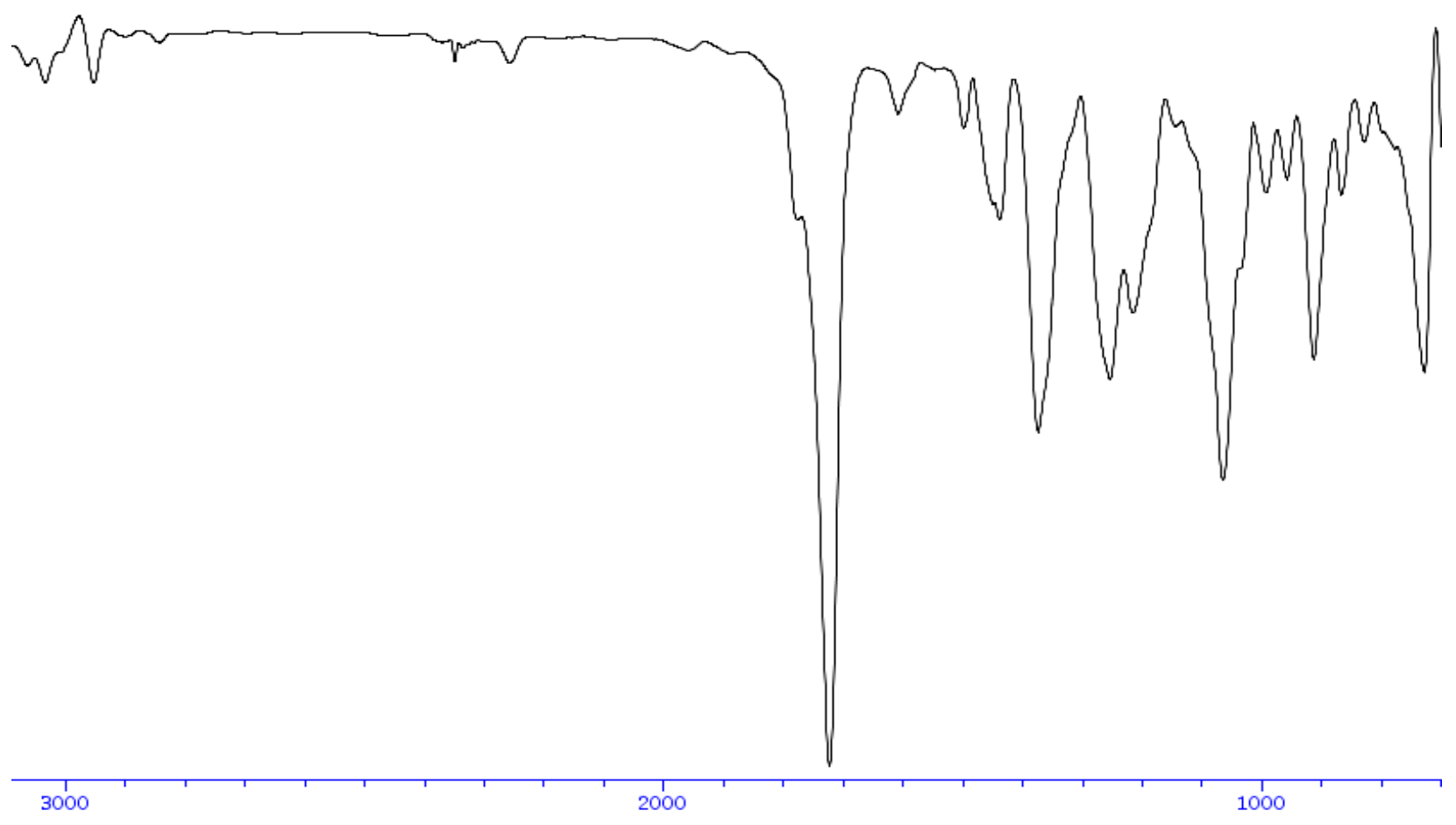




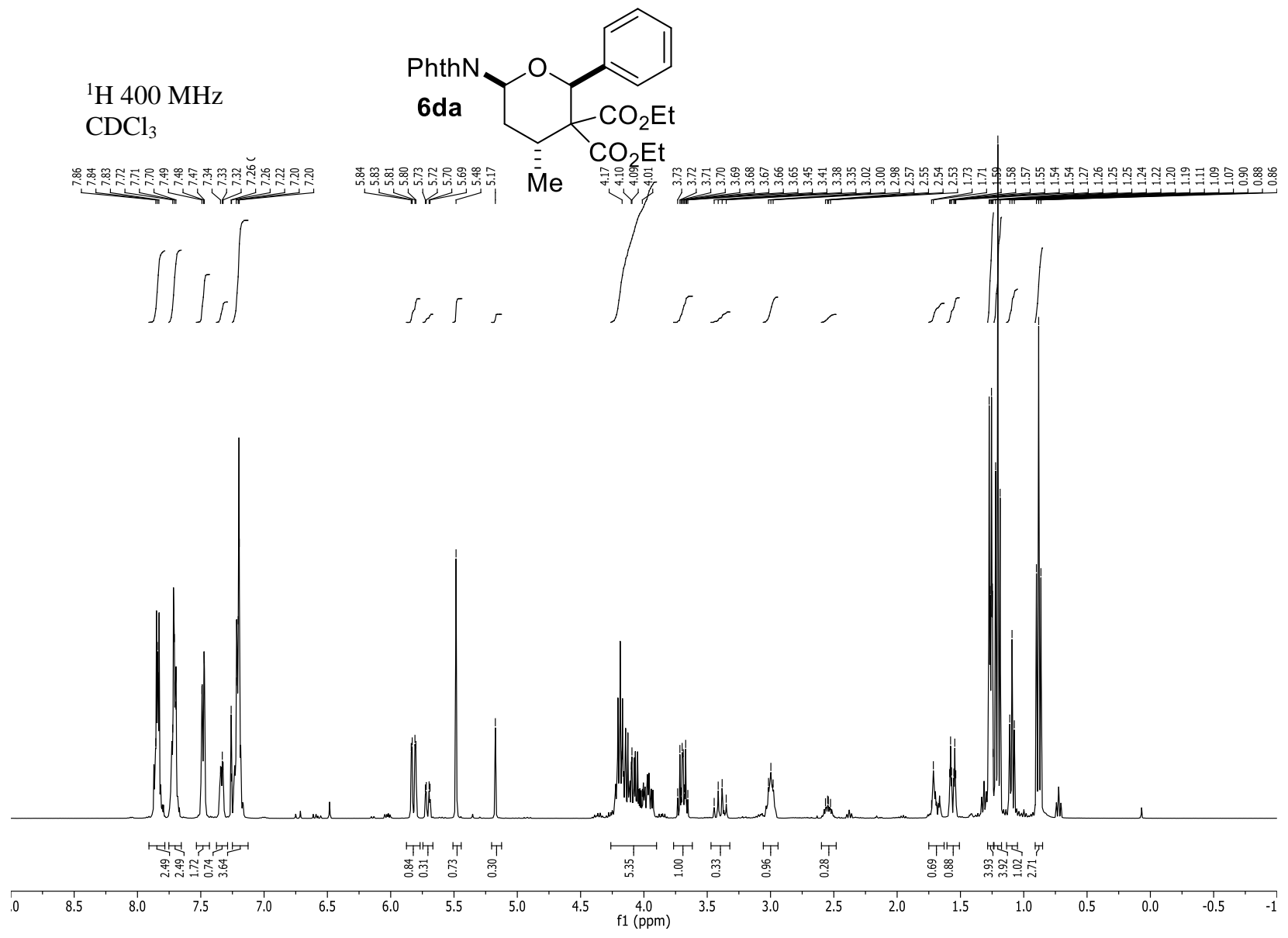

${ }^{13} \mathrm{C} 100 \mathrm{MHz}$ $\mathrm{CDCl}_{3}$

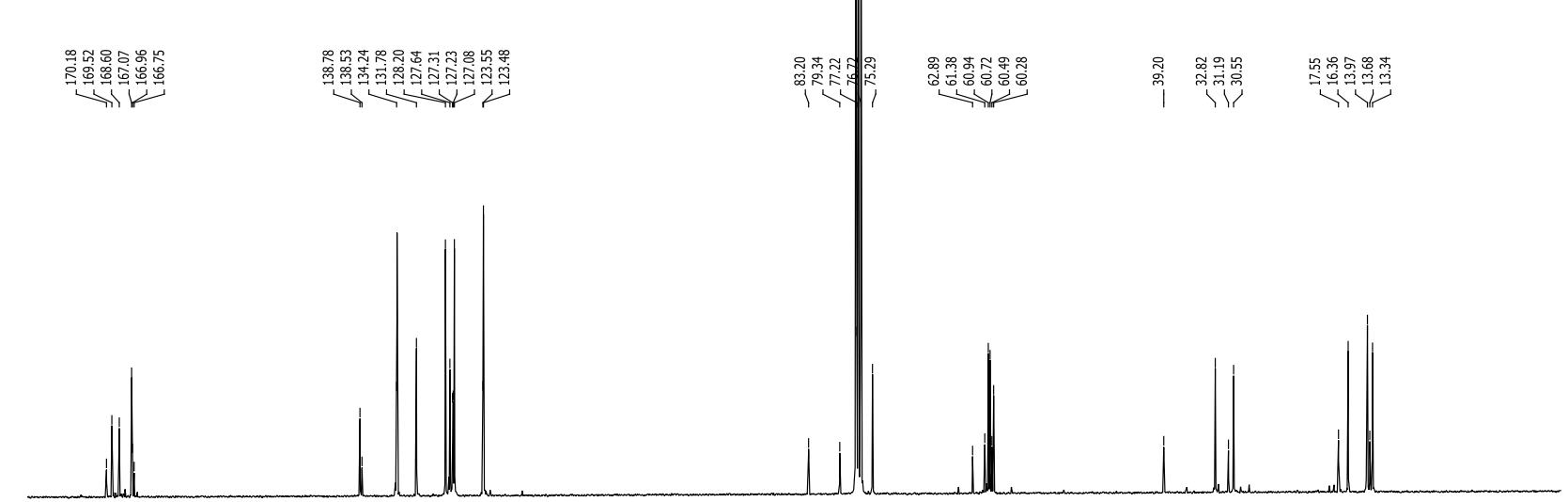

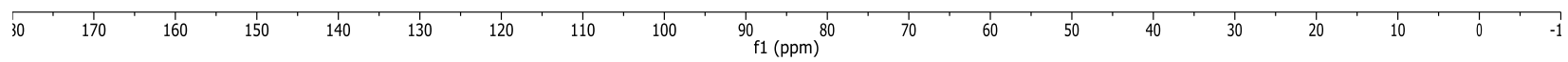



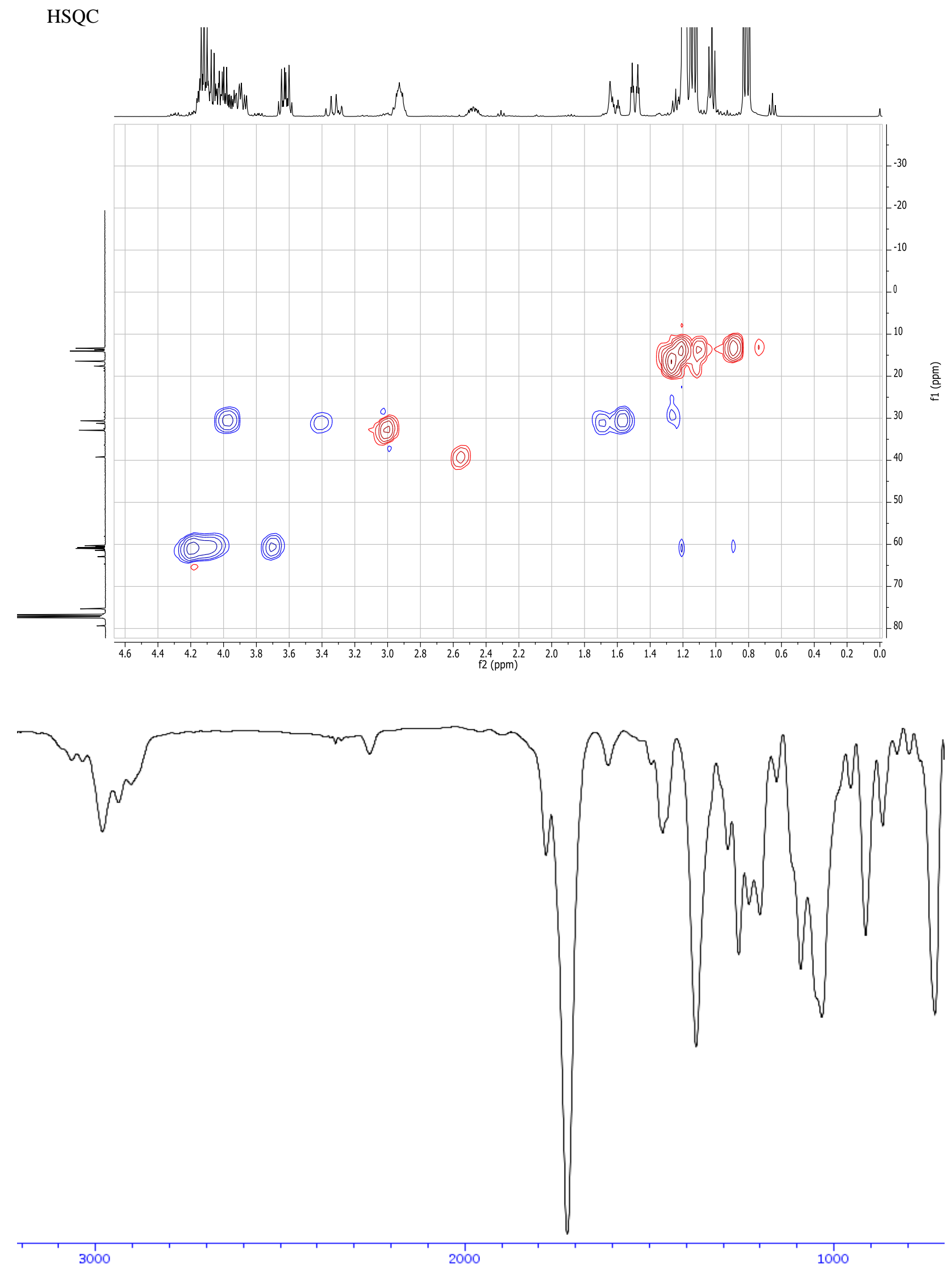


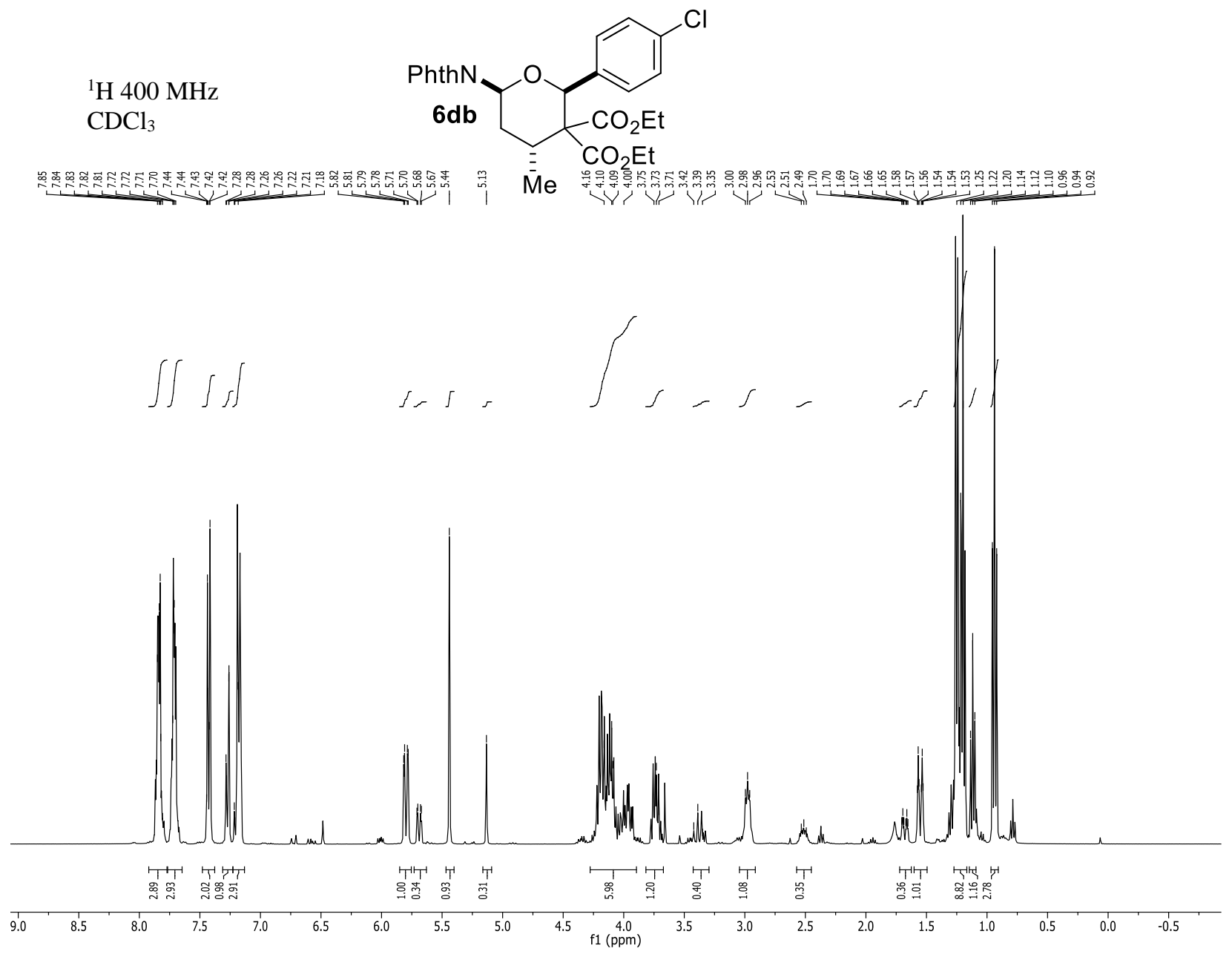

${ }^{13} \mathrm{C} 100 \mathrm{MHz}$ $\mathrm{CDCl}_{3}$
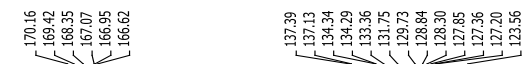

네

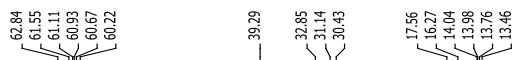
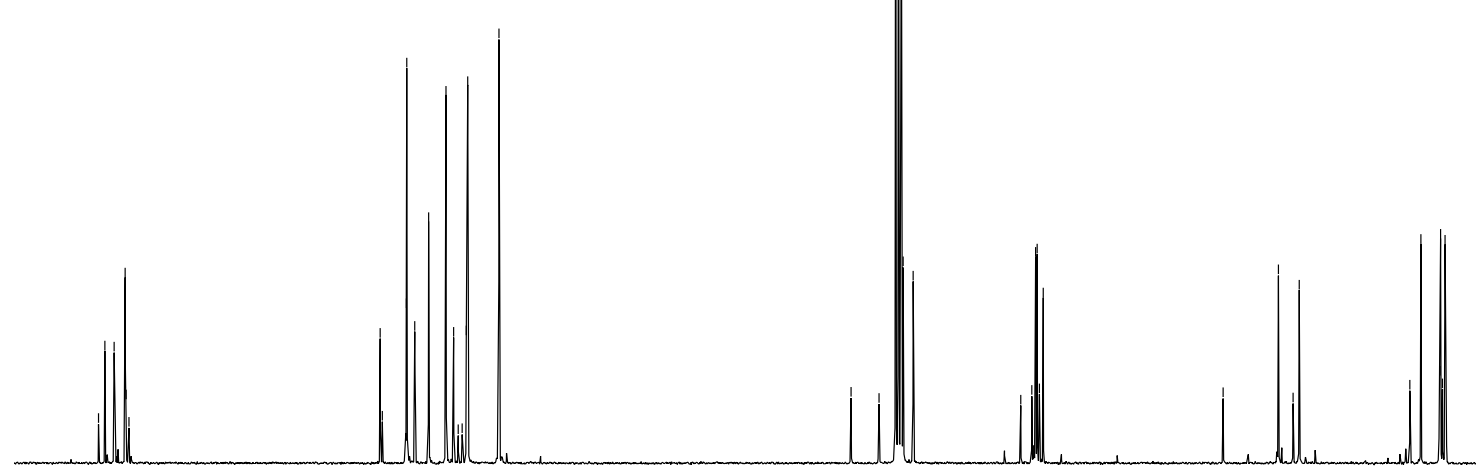

30

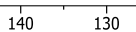

${ }^{90} 1(\mathrm{ppm}){ }^{80}$ 
HSQC
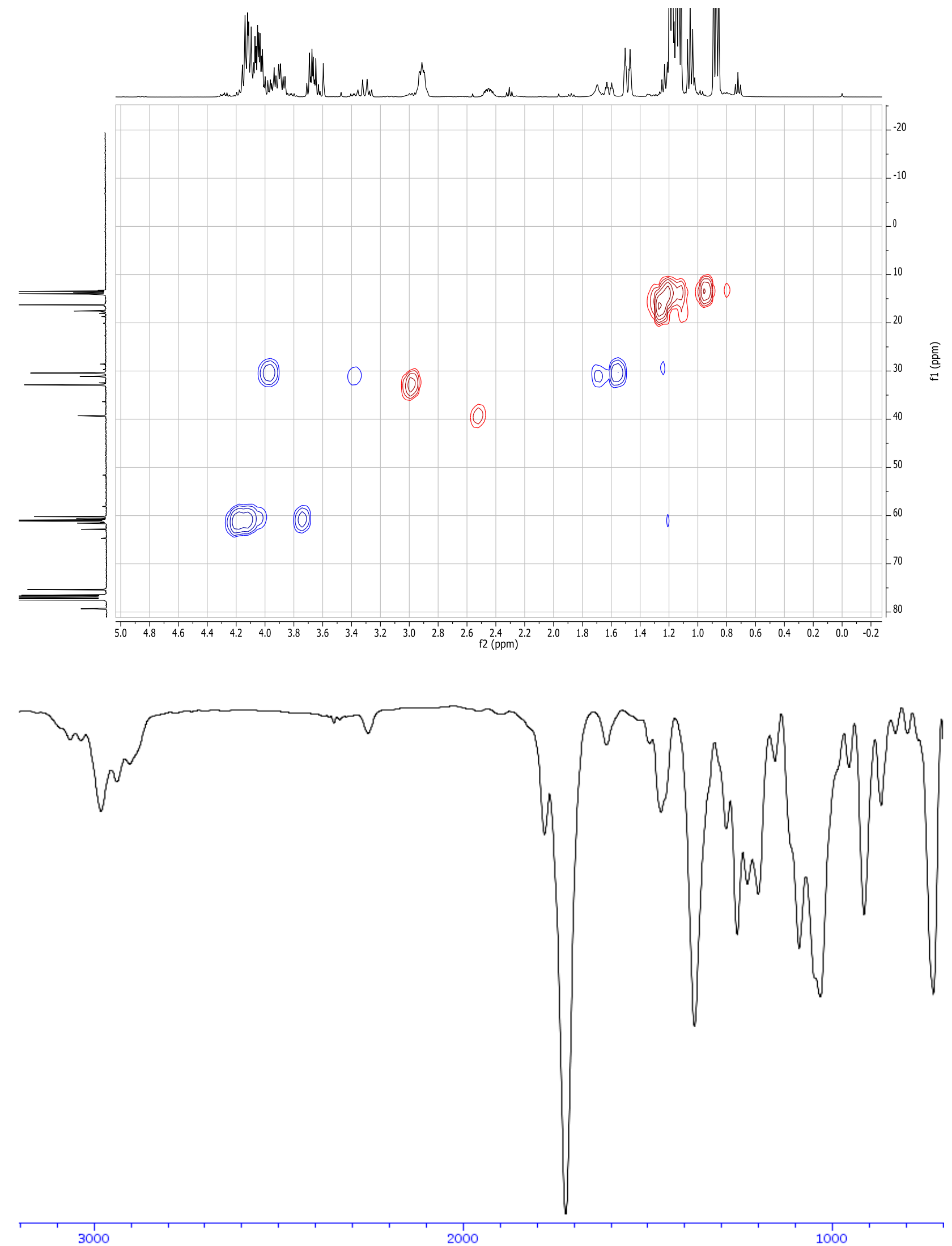
ROESY

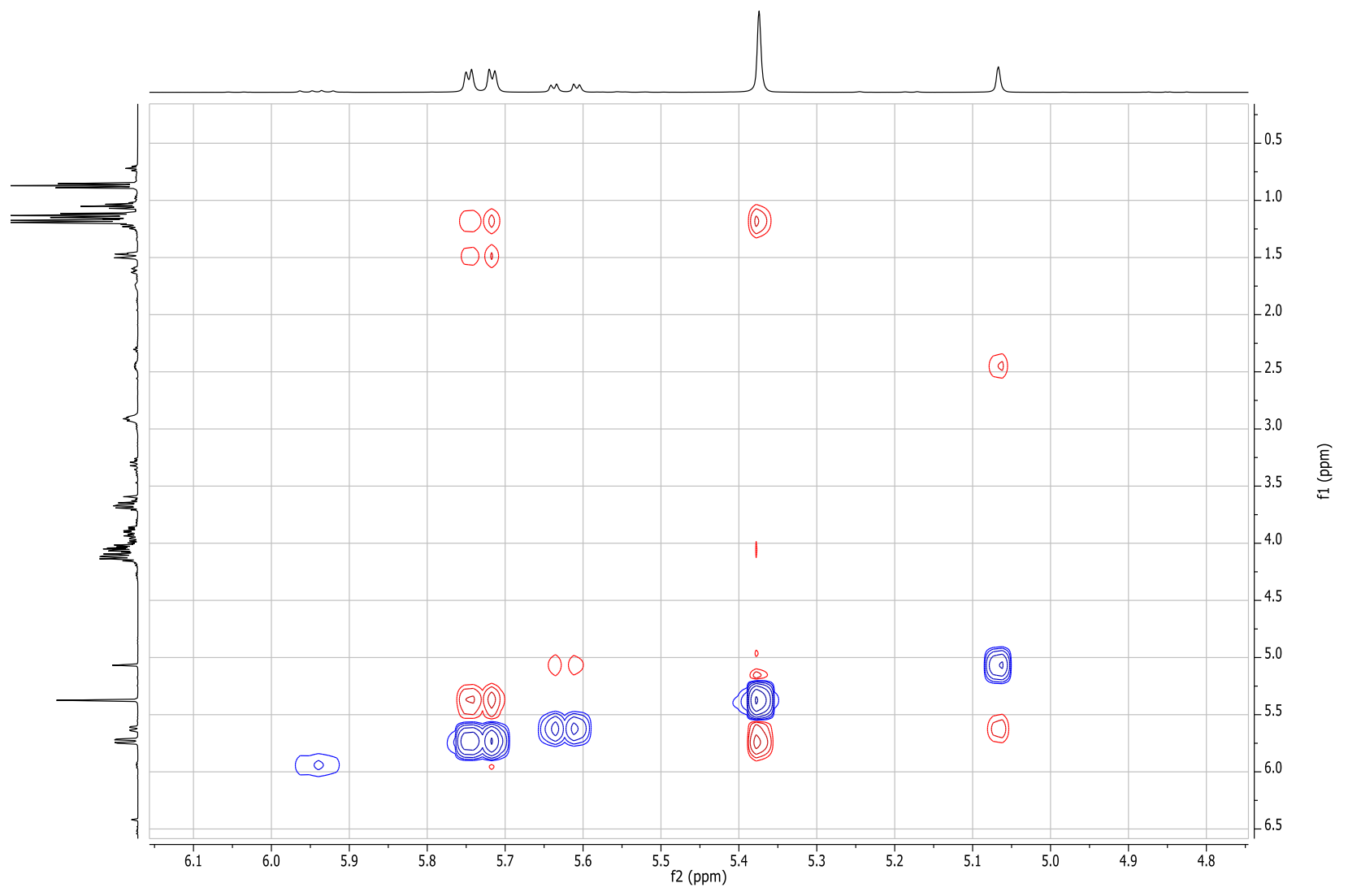




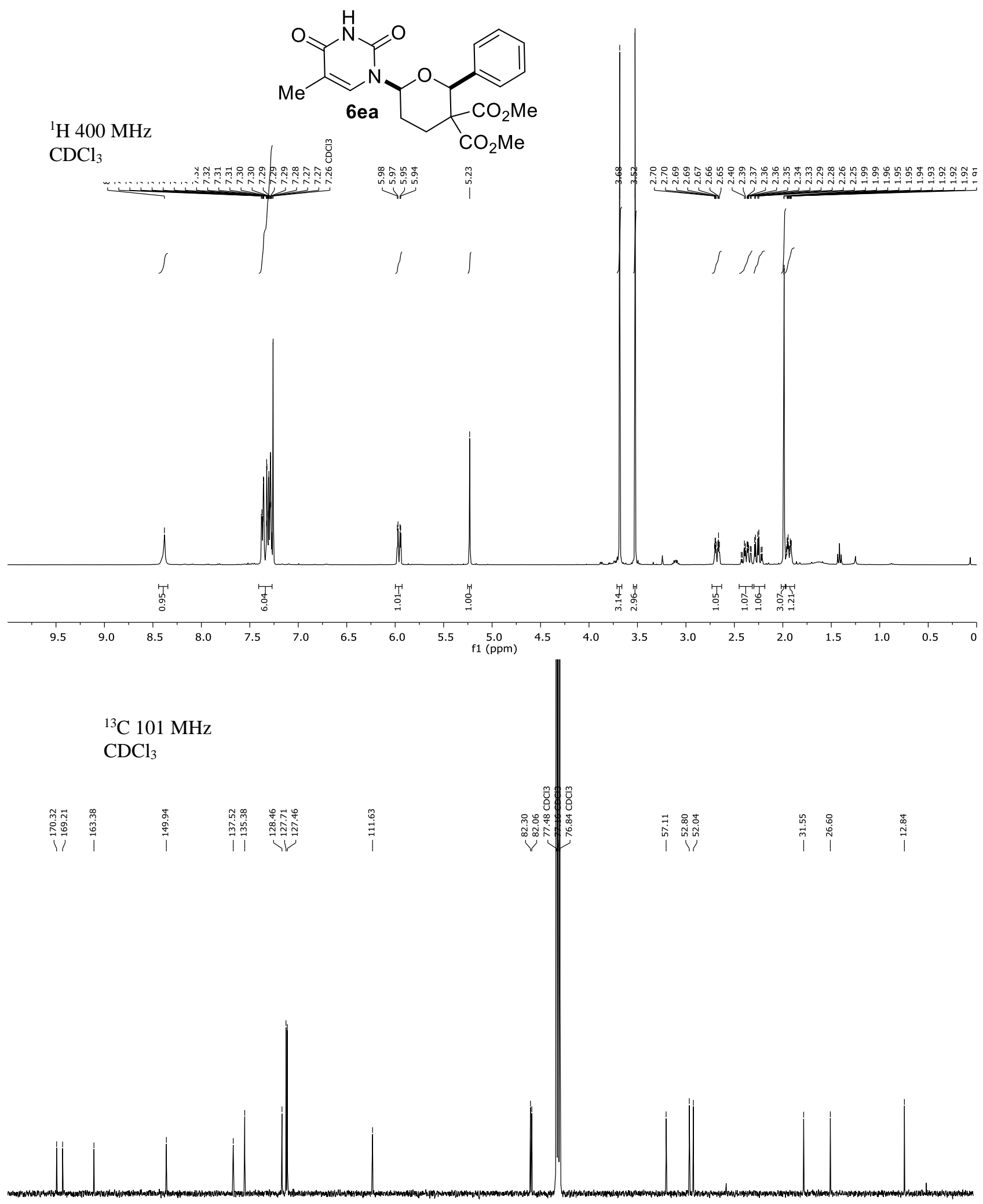




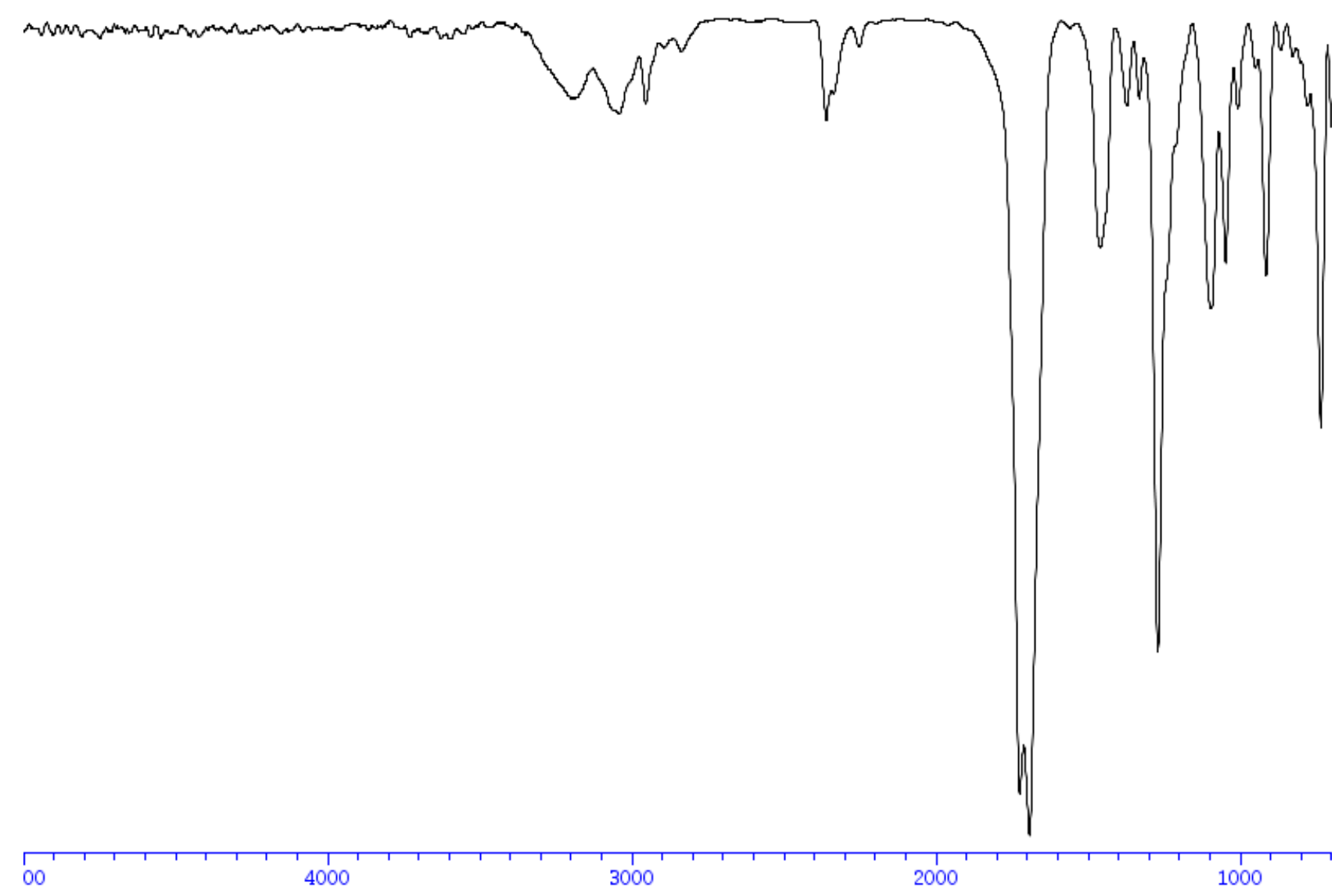




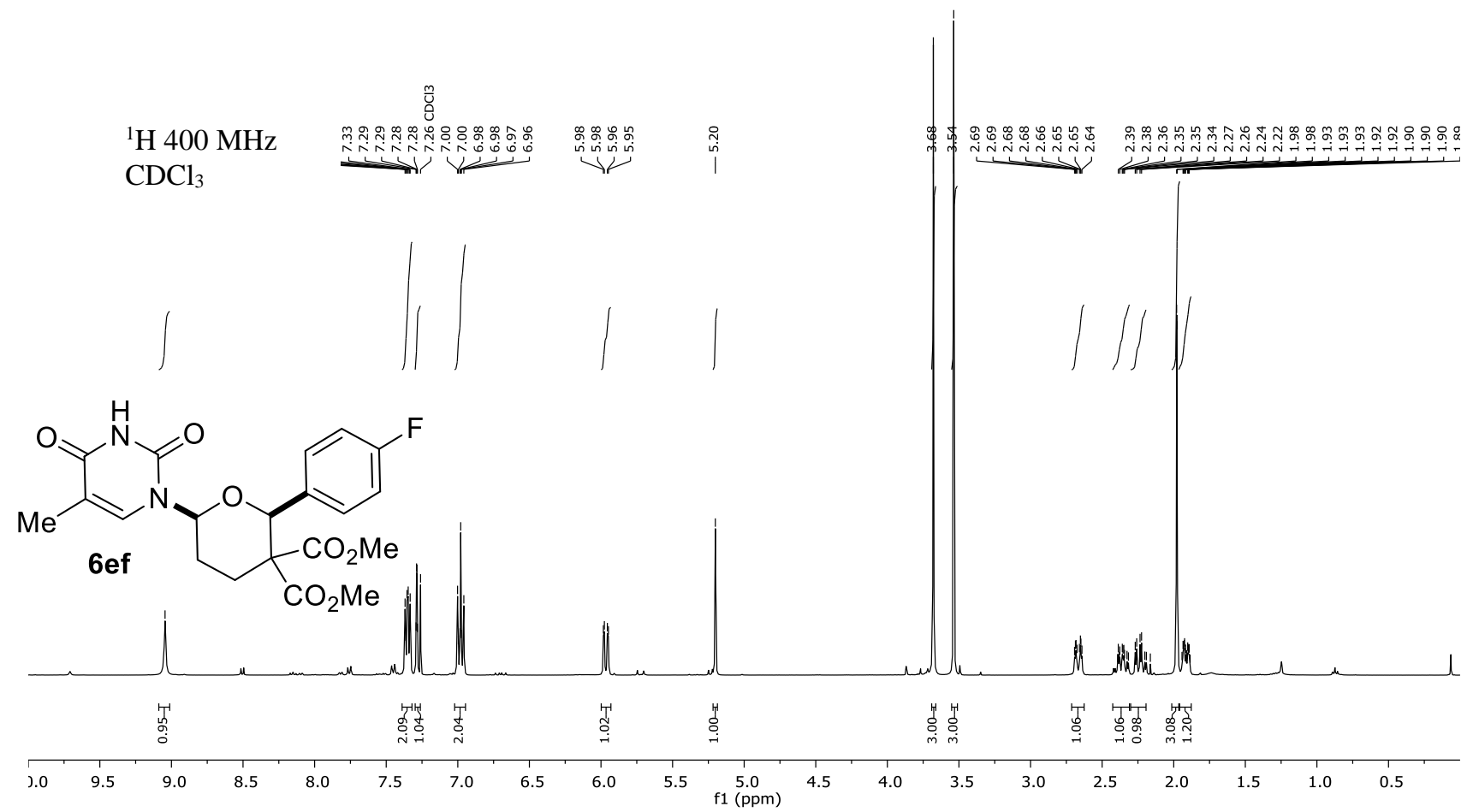

${ }^{13} \mathrm{C} 101 \mathrm{MHz}$ $\mathrm{CDCl}_{3}$

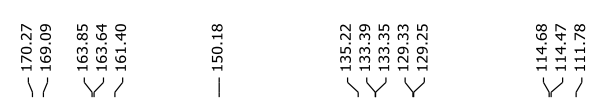

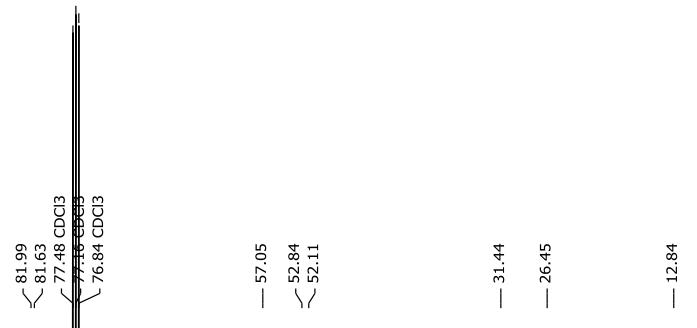

\begin{tabular}{|c|c|c|c|c|c|c|c|c|c|c|c|c|c|c|c|c|c|}
\hline 80 & 170 & 160 & $\begin{array}{l}1 \\
150\end{array}$ & 140 & 130 & $\begin{array}{l}1 \\
120\end{array}$ & 110 & 100 & 90 & 80 & $\begin{array}{l}1 \\
70\end{array}$ & 60 & $\begin{array}{l}1 \\
50\end{array}$ & 40 & 30 & 20 & 10 \\
\hline
\end{tabular}




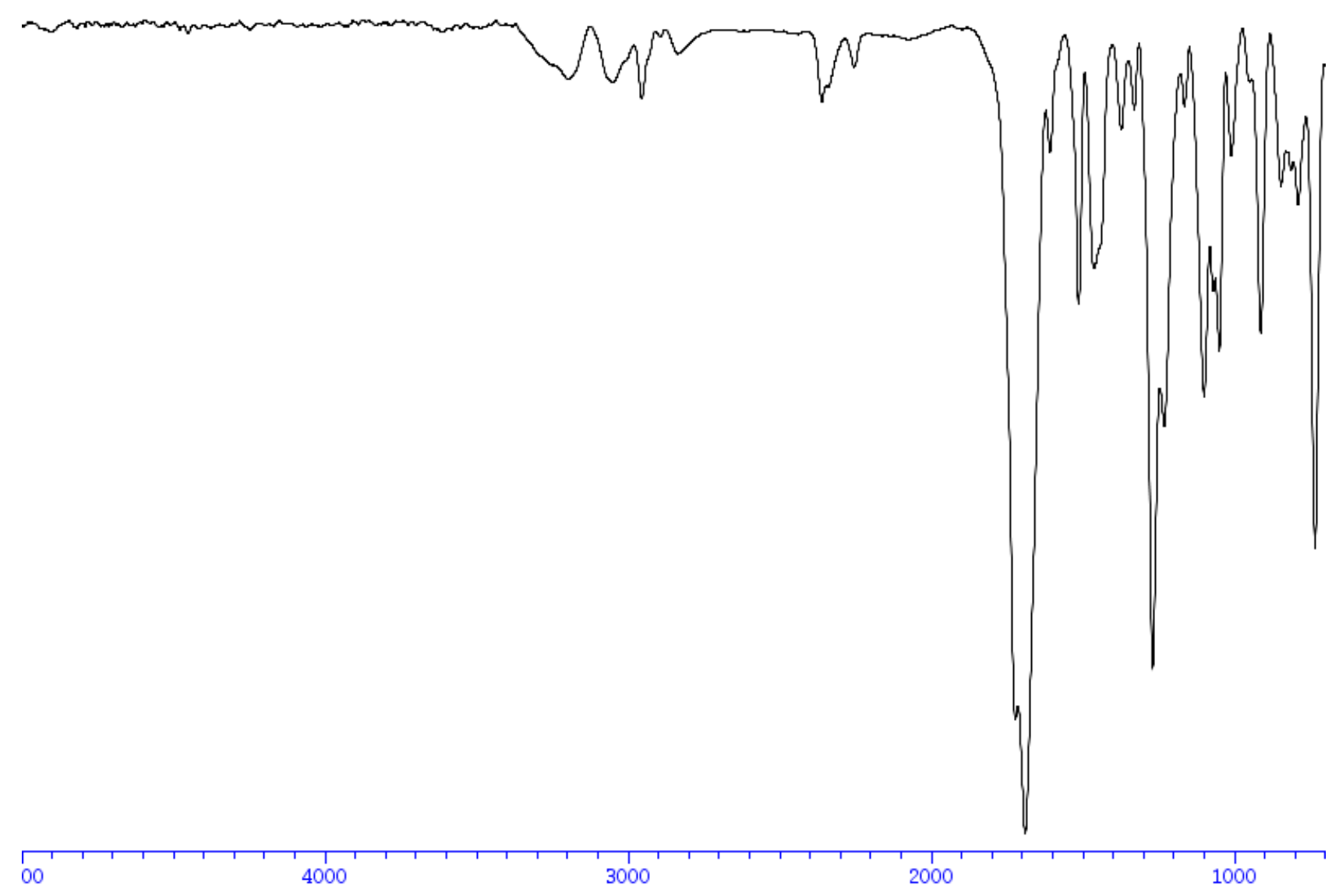




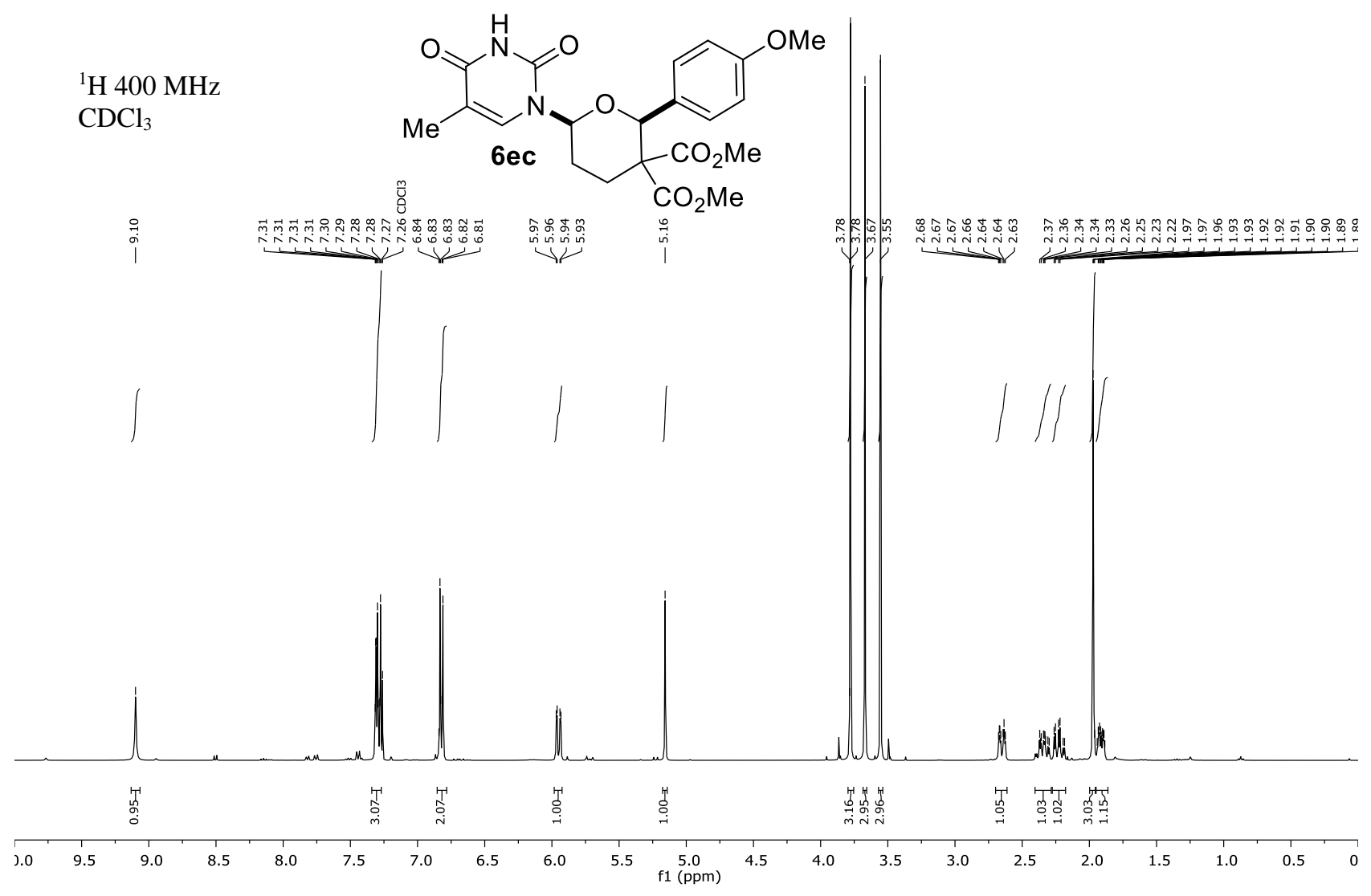

${ }^{13} \mathrm{C} 101 \mathrm{MHz}$ $\mathrm{CDCl}_{3}$

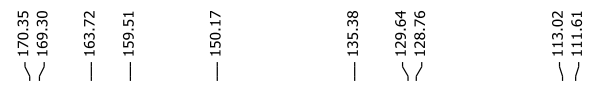
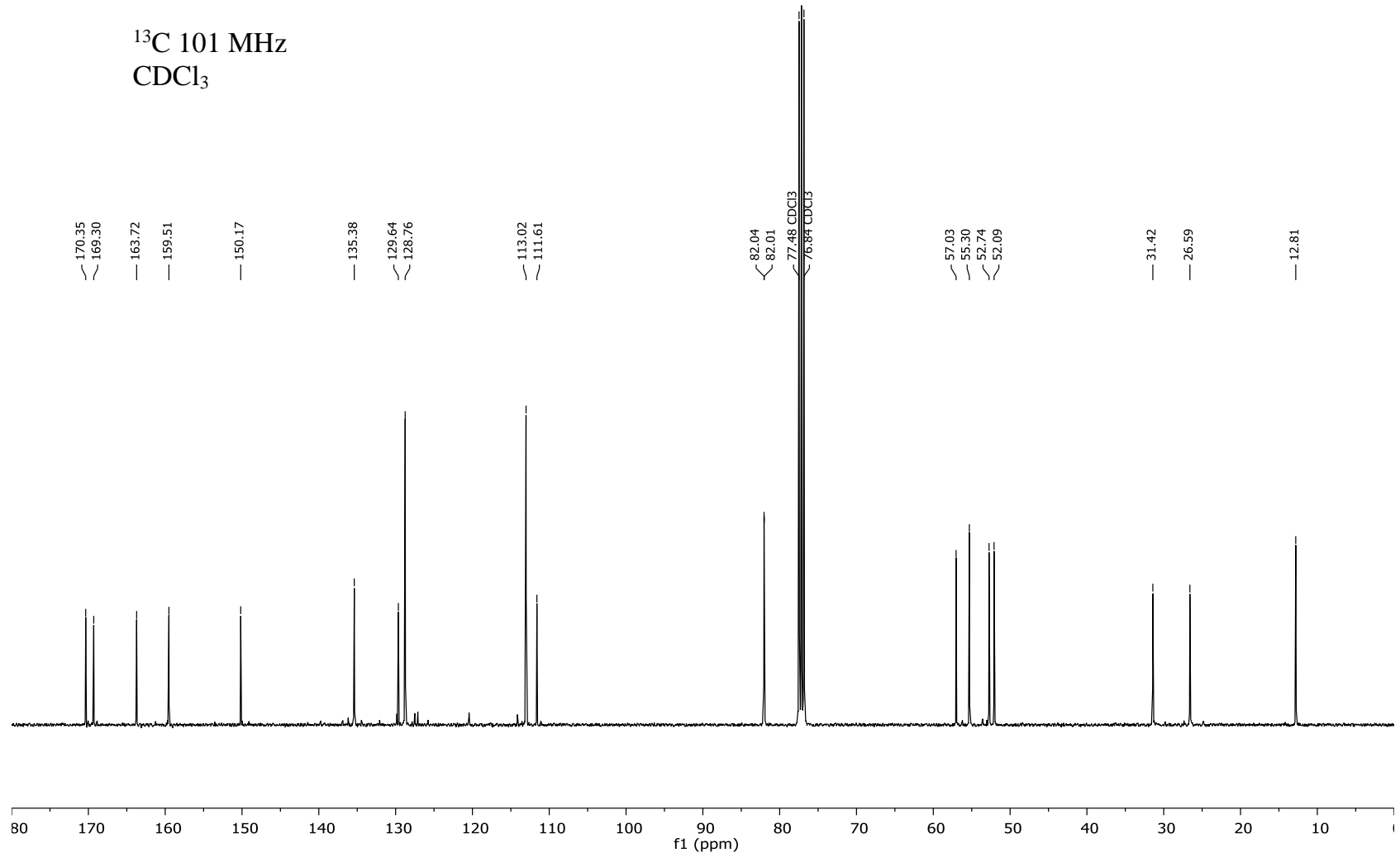


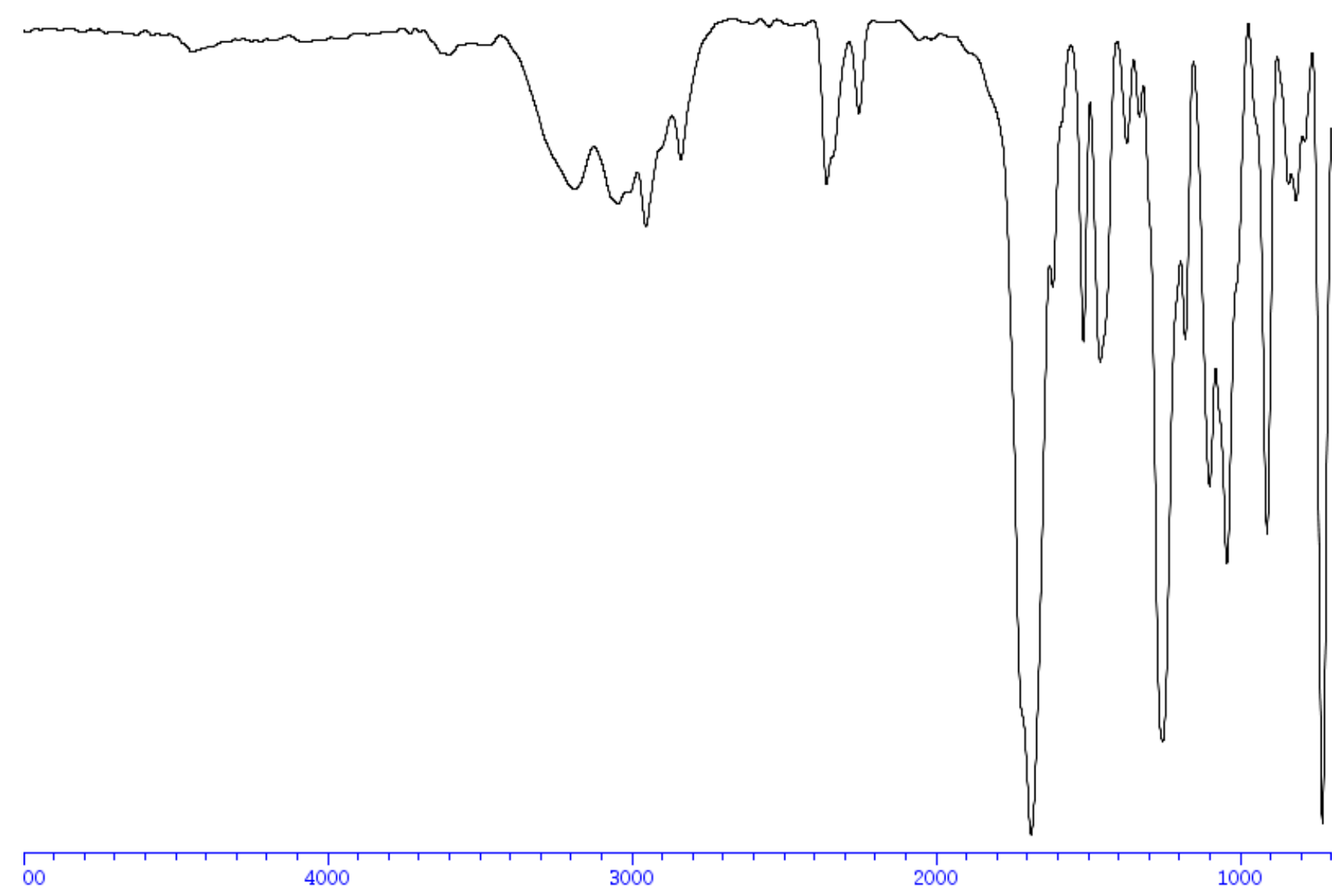



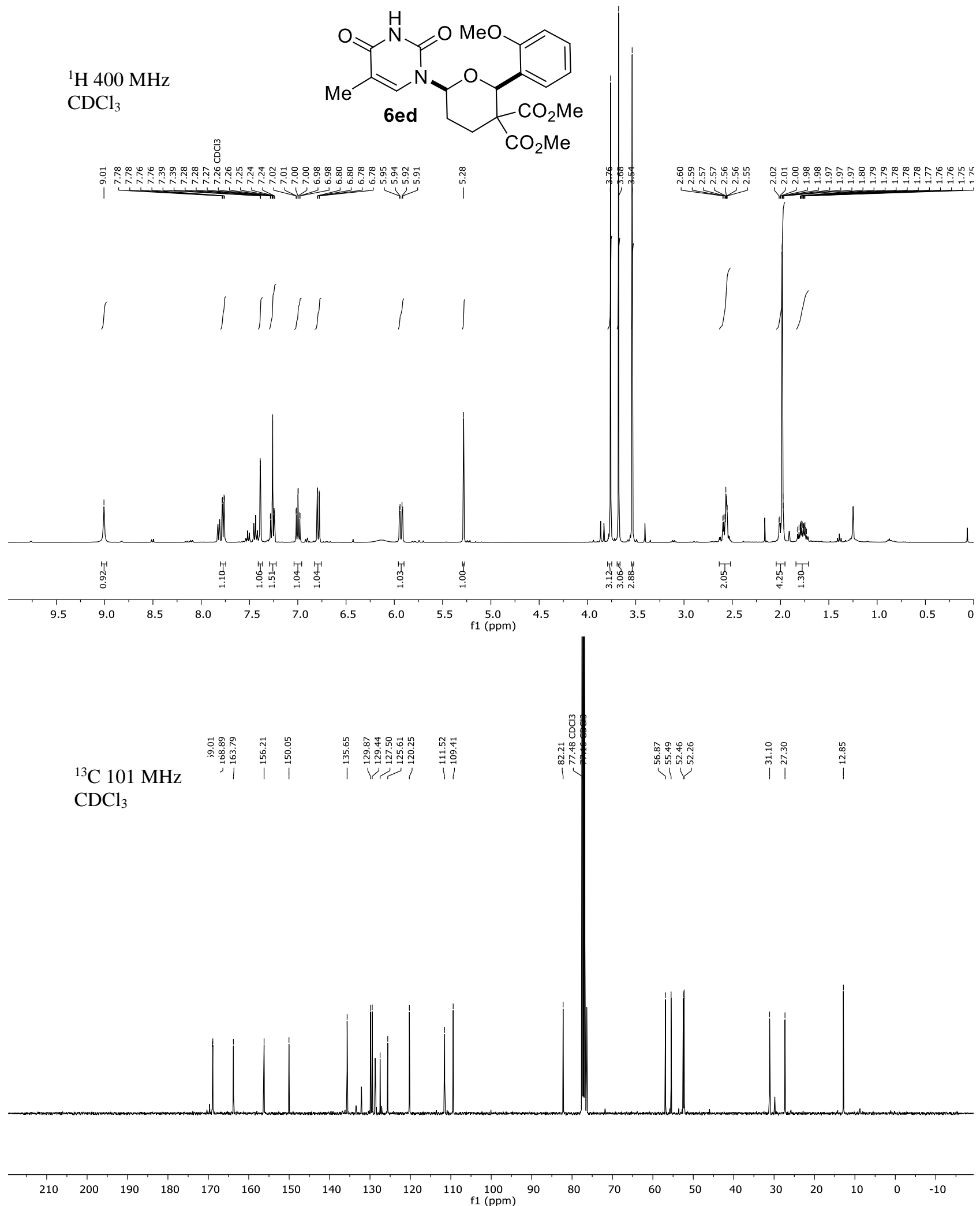


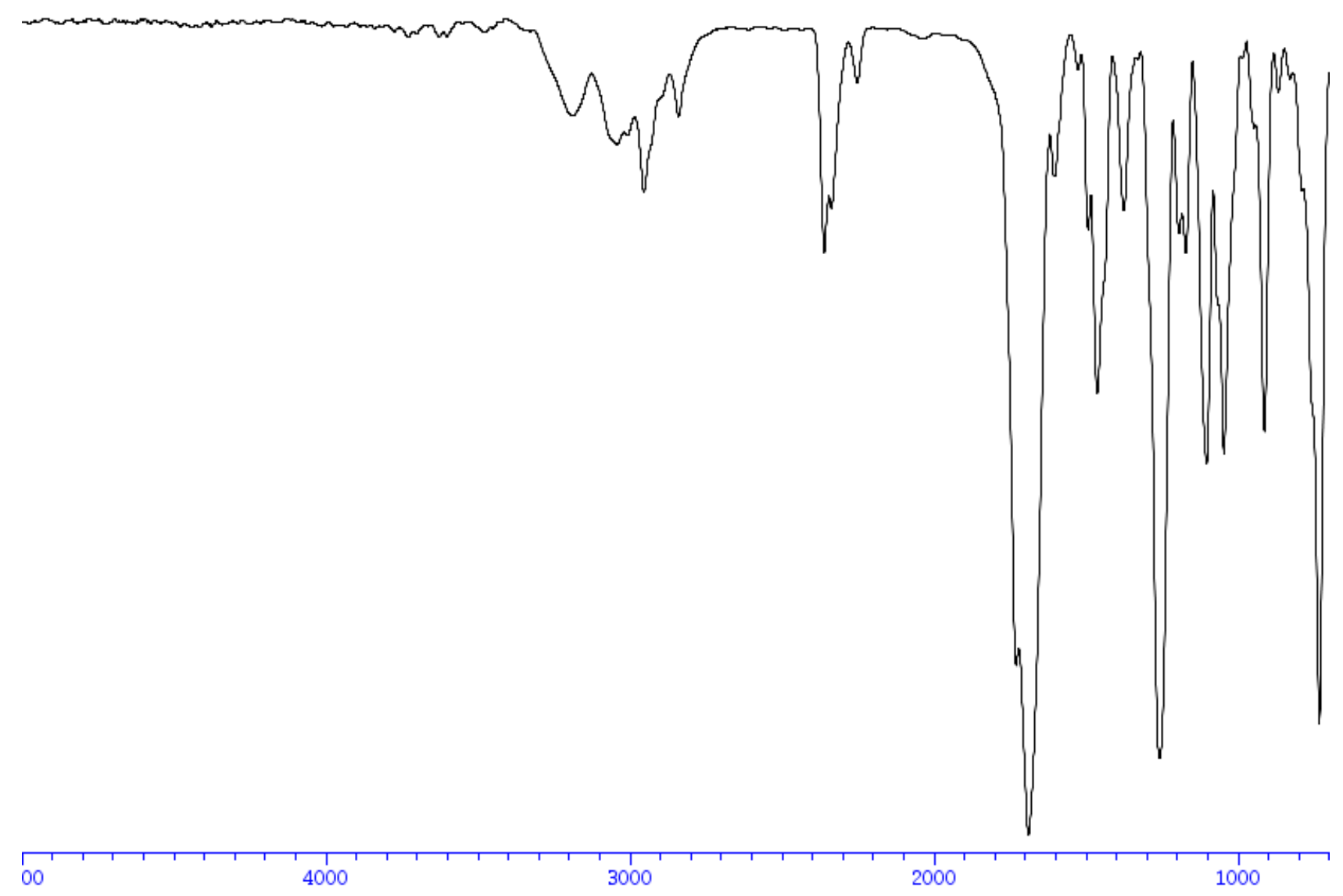



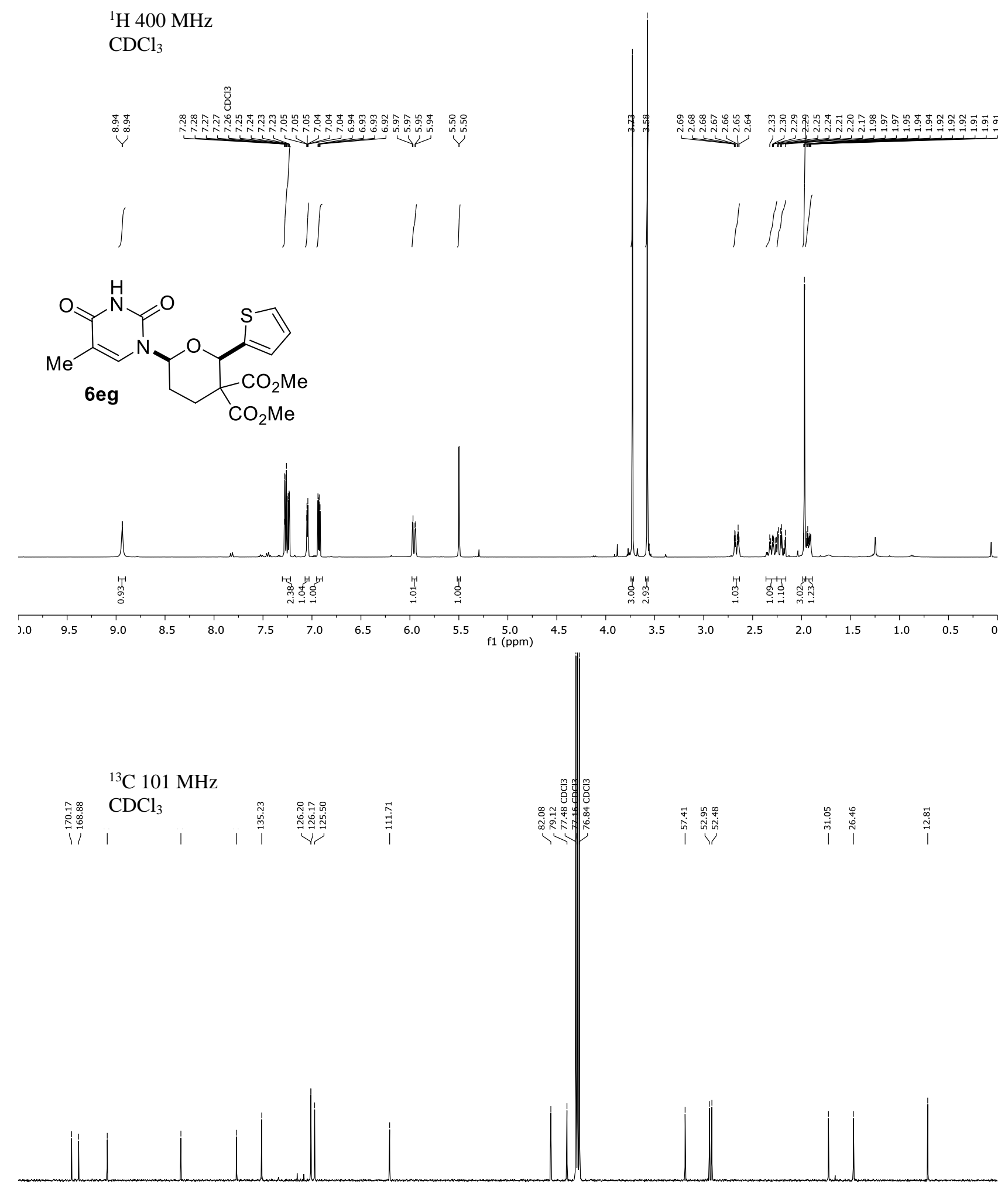

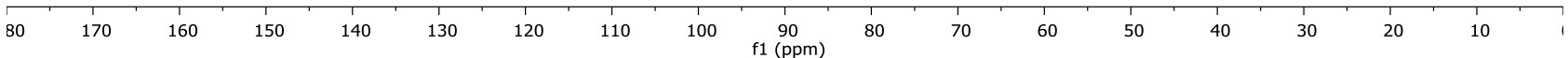




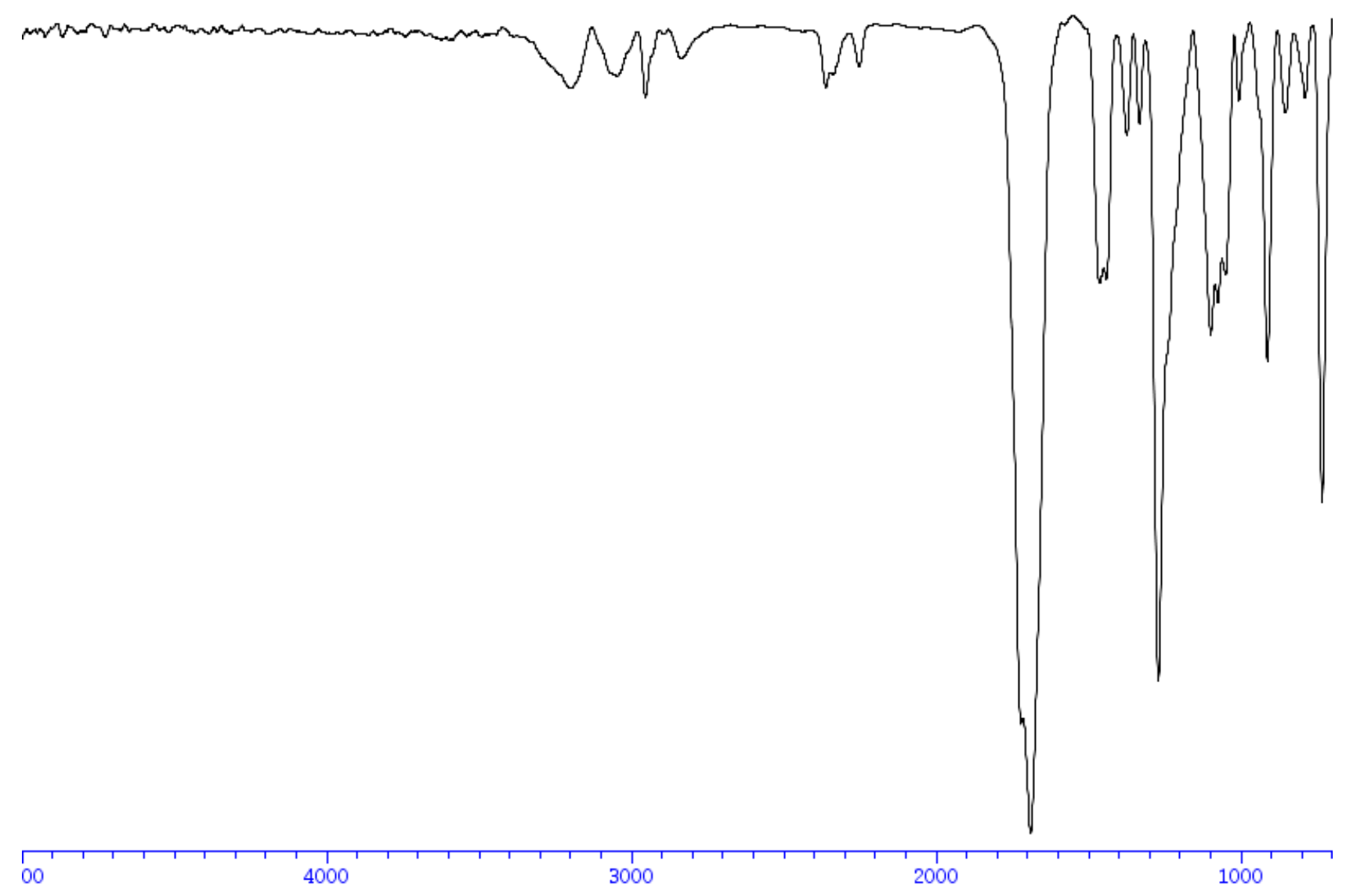




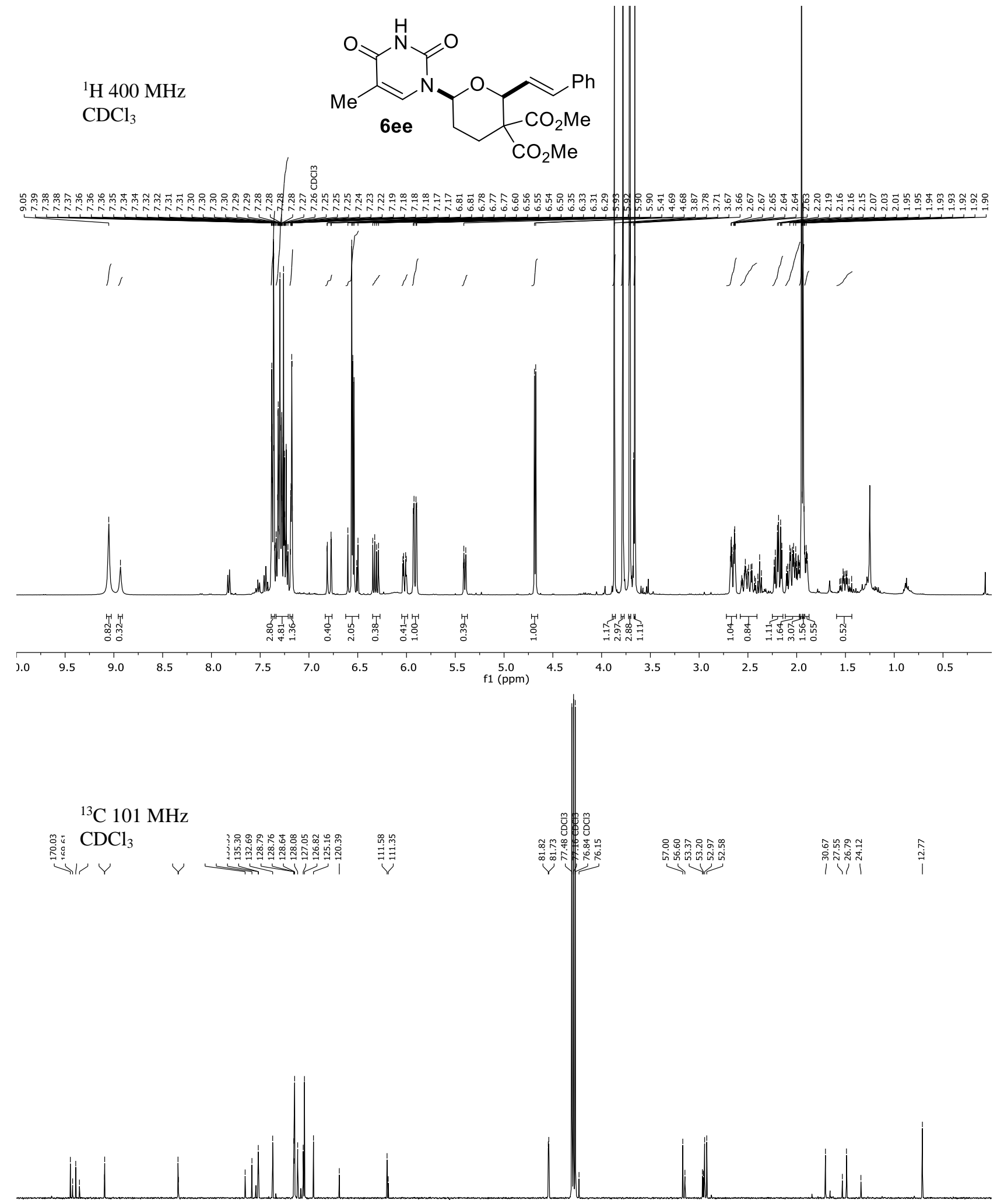

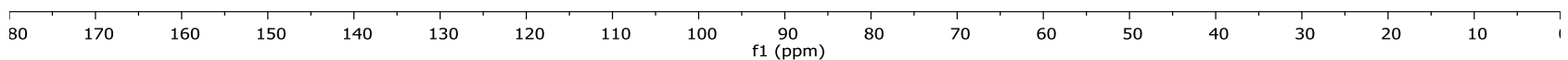




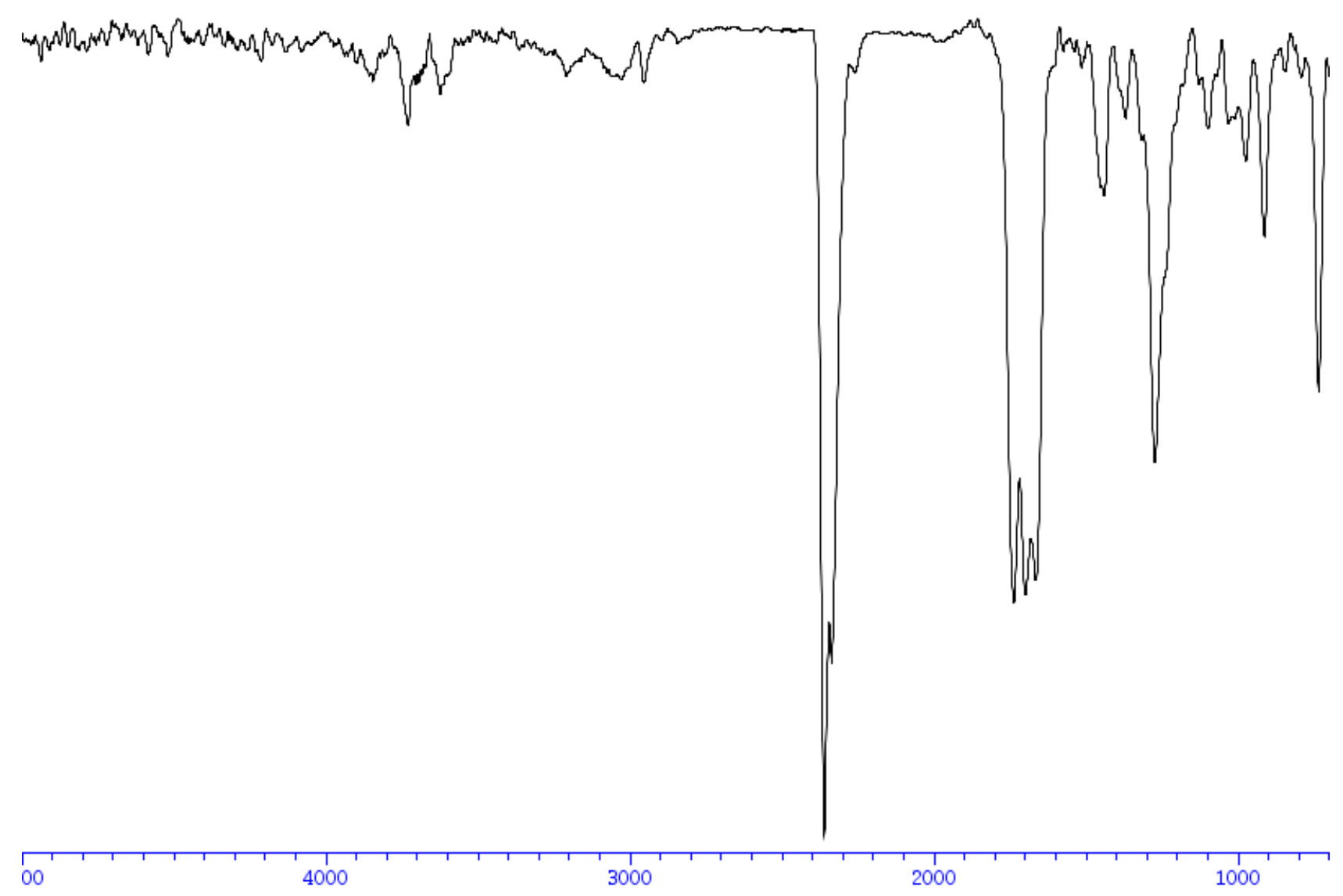




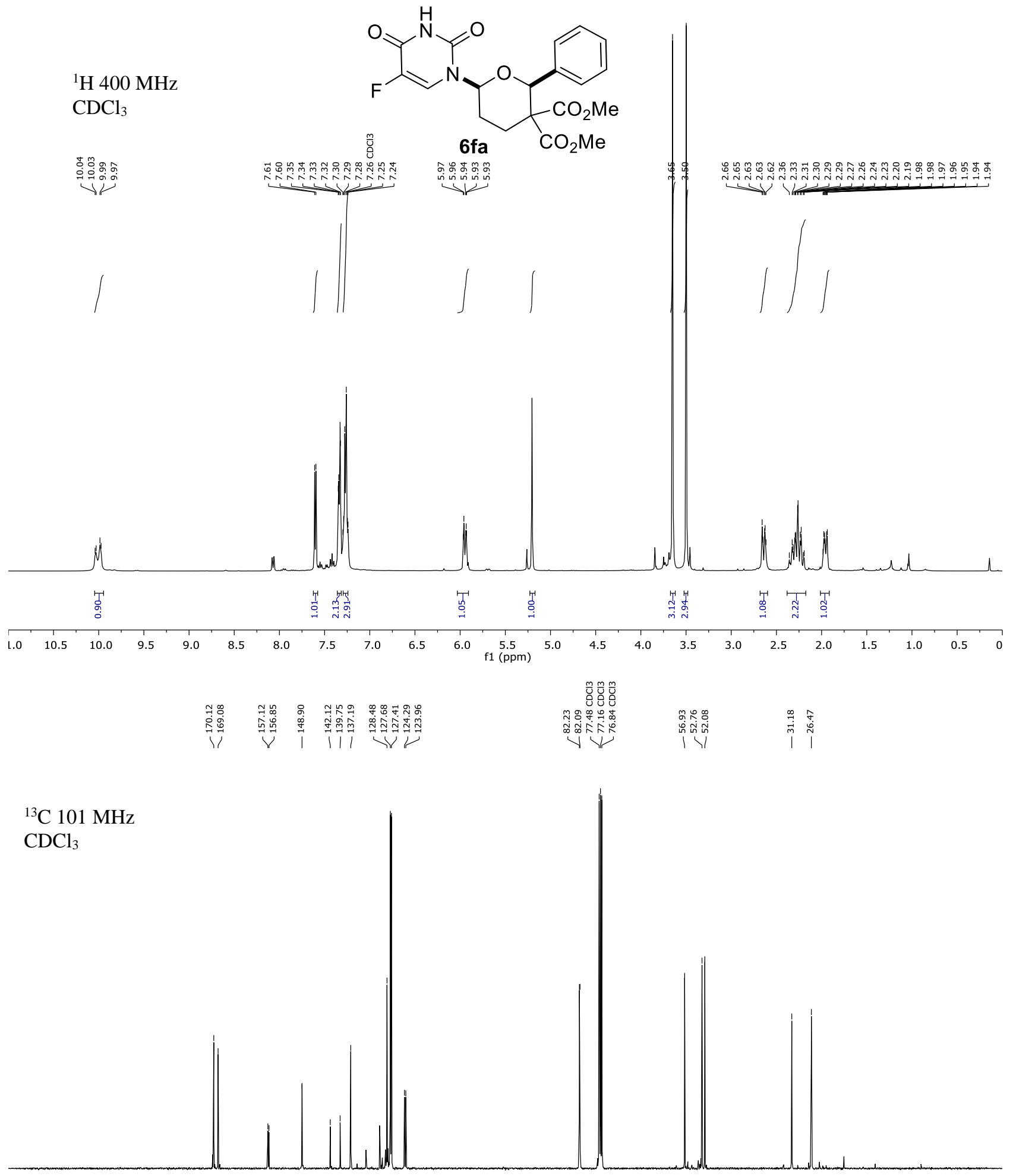




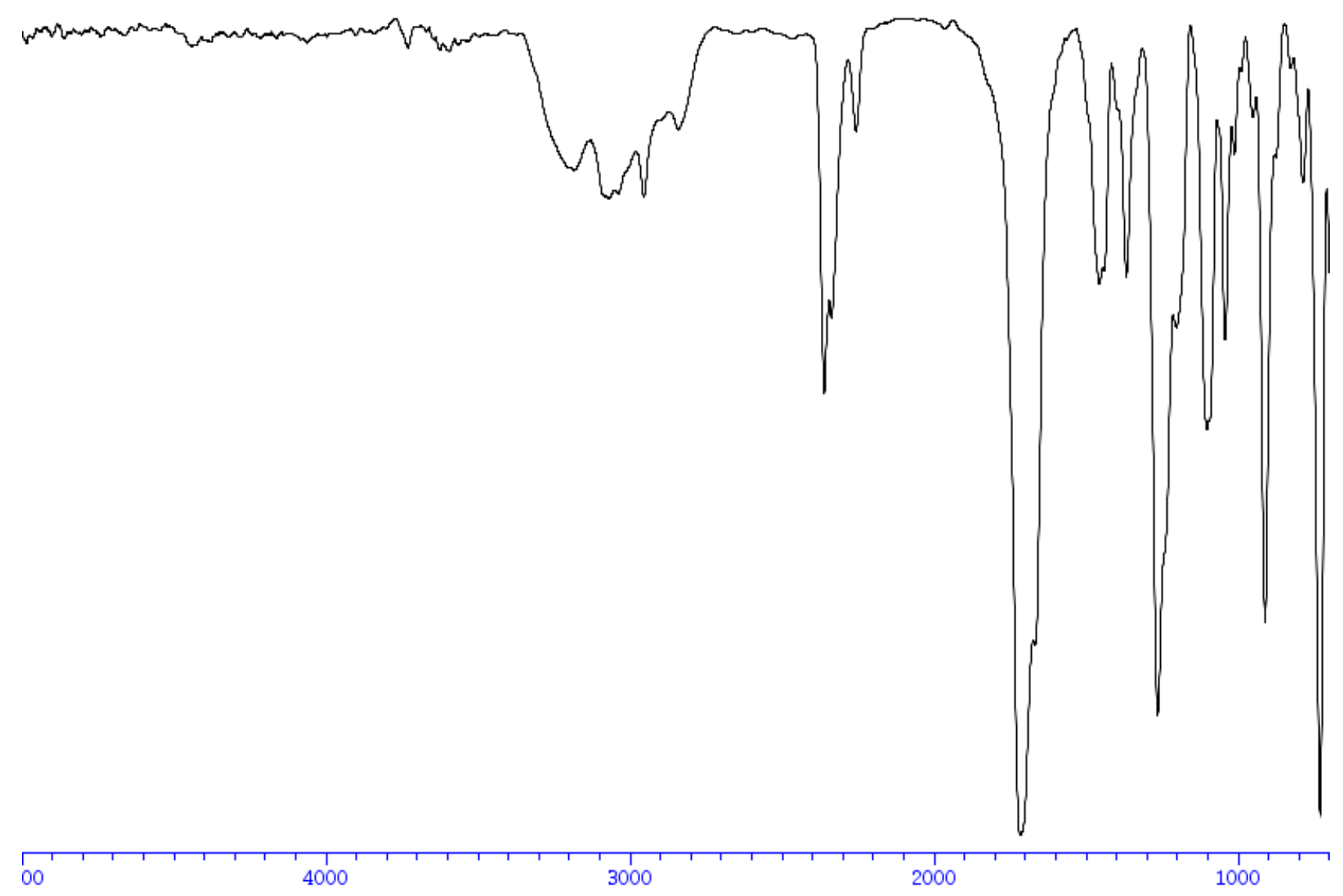



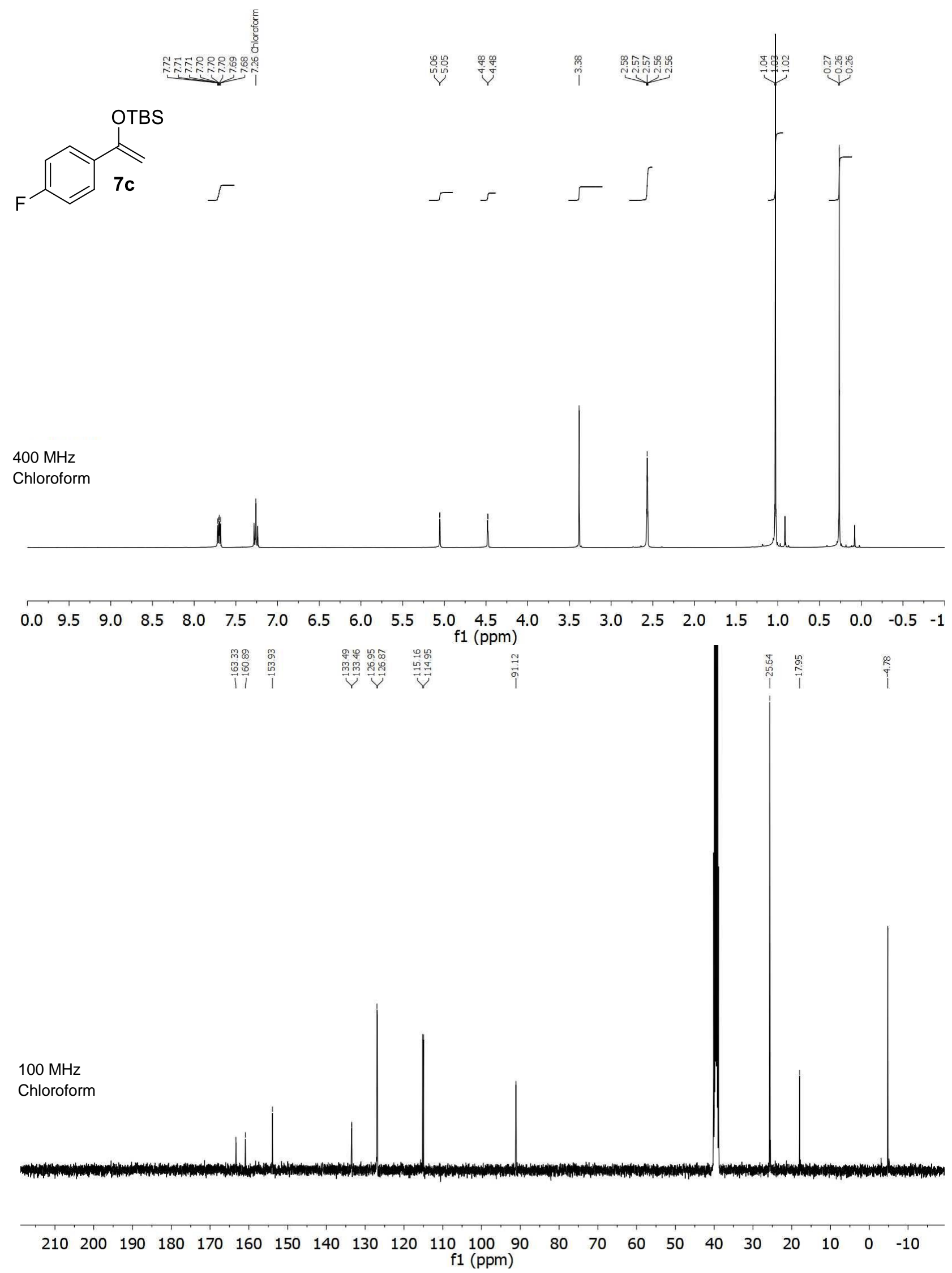


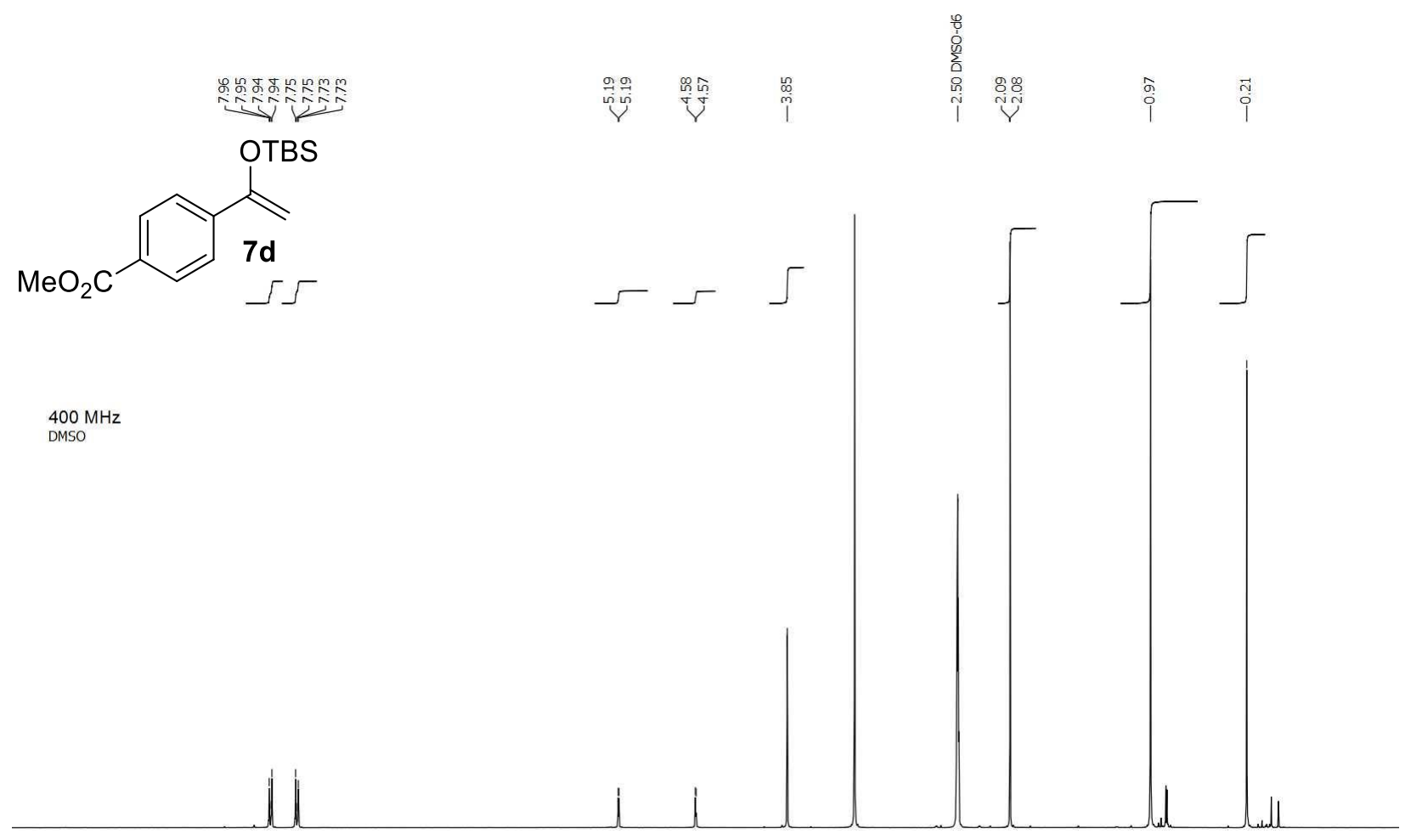

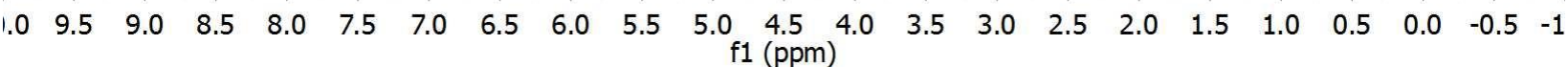

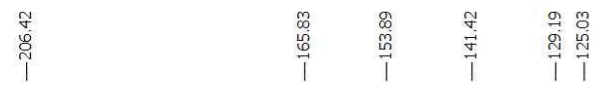

$100 \mathrm{MHz}$
DMSO

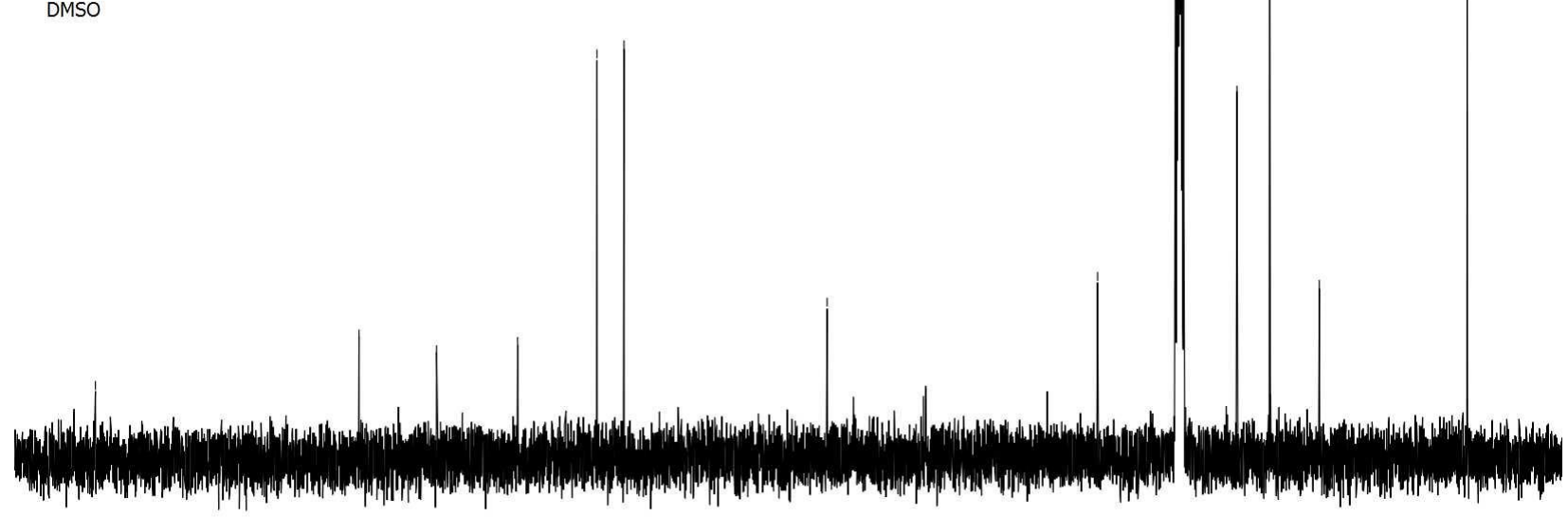

$\begin{array}{lllllllllllllllllllllll}210 & 200 & 190 & 180 & 170 & 160 & 150 & 140 & 130 & 120 & 110 & 100 & 90 & 80 & 70 & 60 & 50 & 40 & 30 & 20 & 10 & 0 & -10\end{array}$ 

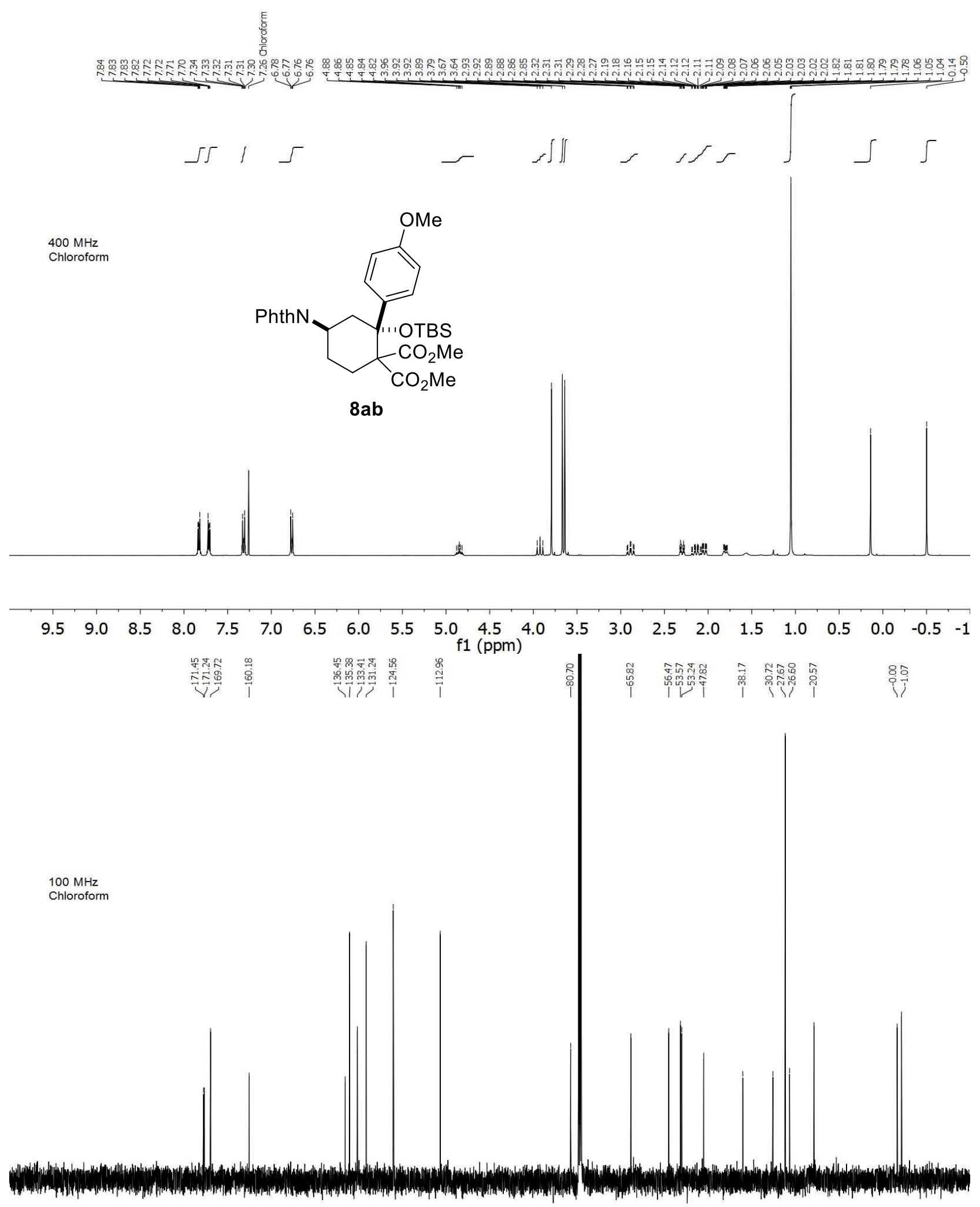

$\begin{array}{lllllllllllllllllllllll}210 & 200 & 190 & 180 & 170 & 160 & 150 & 140 & 130 & 120 & 110 & 100 & 90 & 80 & 70 & 60 & 50 & 40 & 30 & 20 & 10 & 0 & -10\end{array}$ 


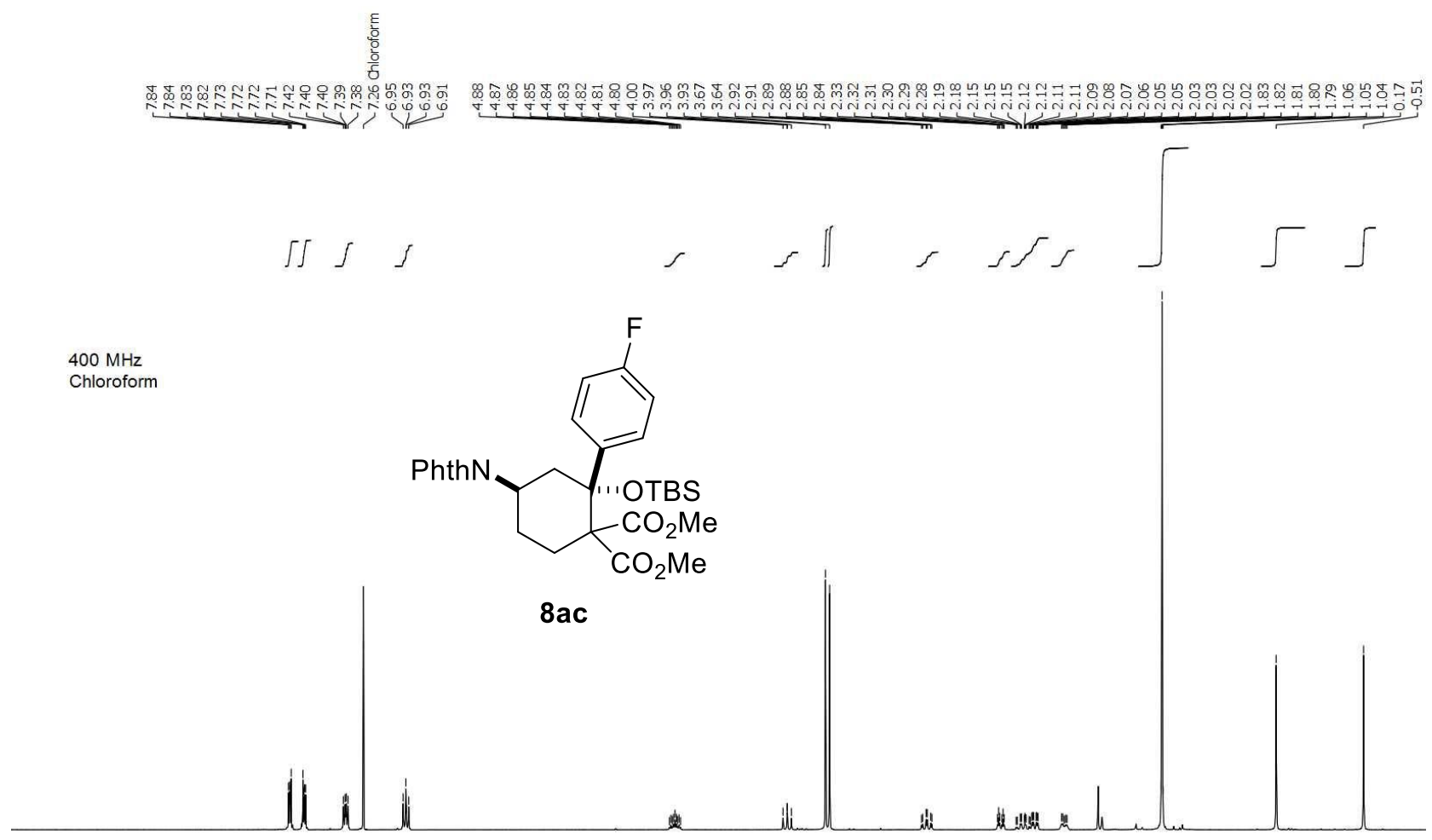

$\begin{array}{lllllllllllllllllllll}9.5 & 9.0 & 8.5 & 8.0 & 7.5 & 7.0 & 6.5 & 6.0 & 5.5 & \begin{array}{c}5.0 \\ \mathrm{f} 1(\mathrm{ppm})\end{array} \underset{(\mathrm{pm})}{4.0} & 3.5 & 3.0 & 2.5 & 2.0 & 1.5 & 1.0 & 0.5 & 0.0 & -0.5\end{array}$

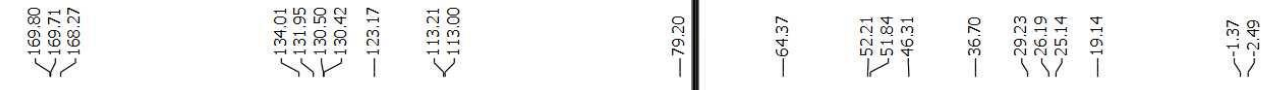

$100 \mathrm{MHz}$

Chloroform

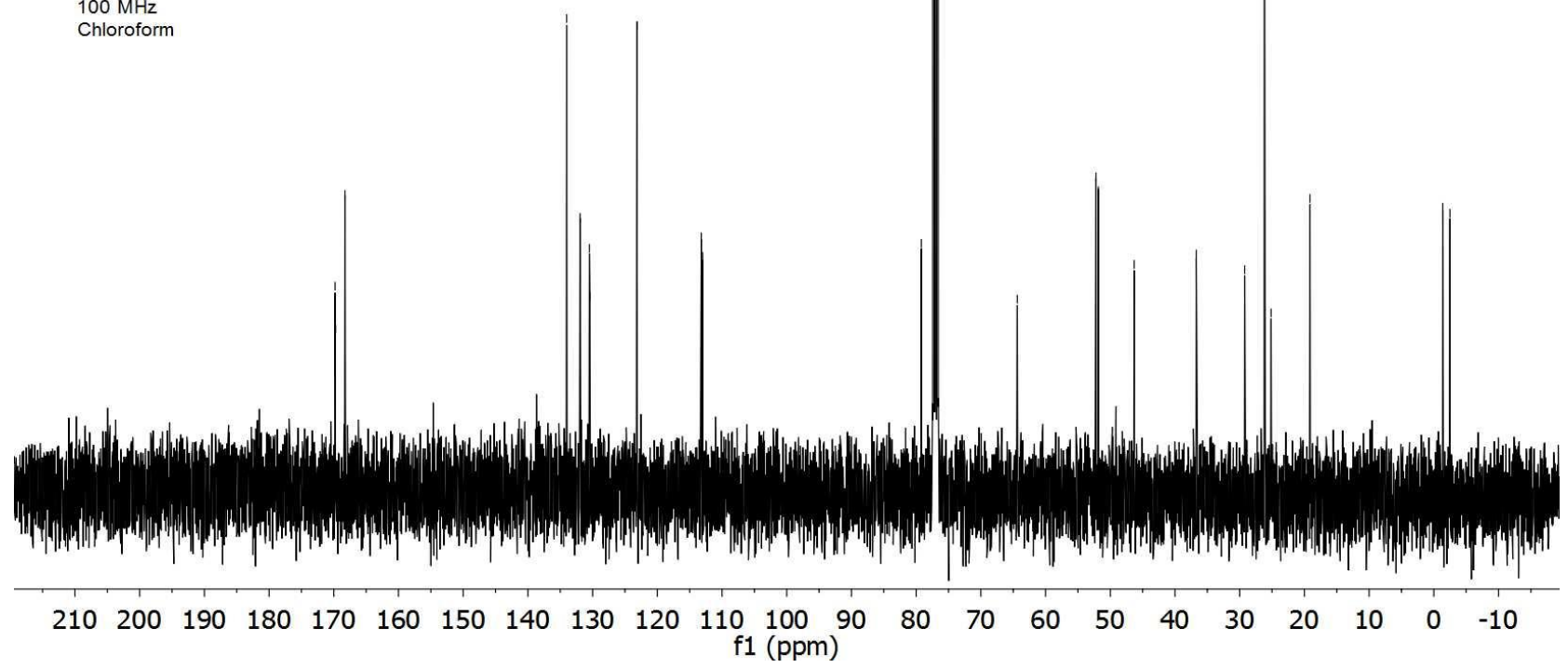




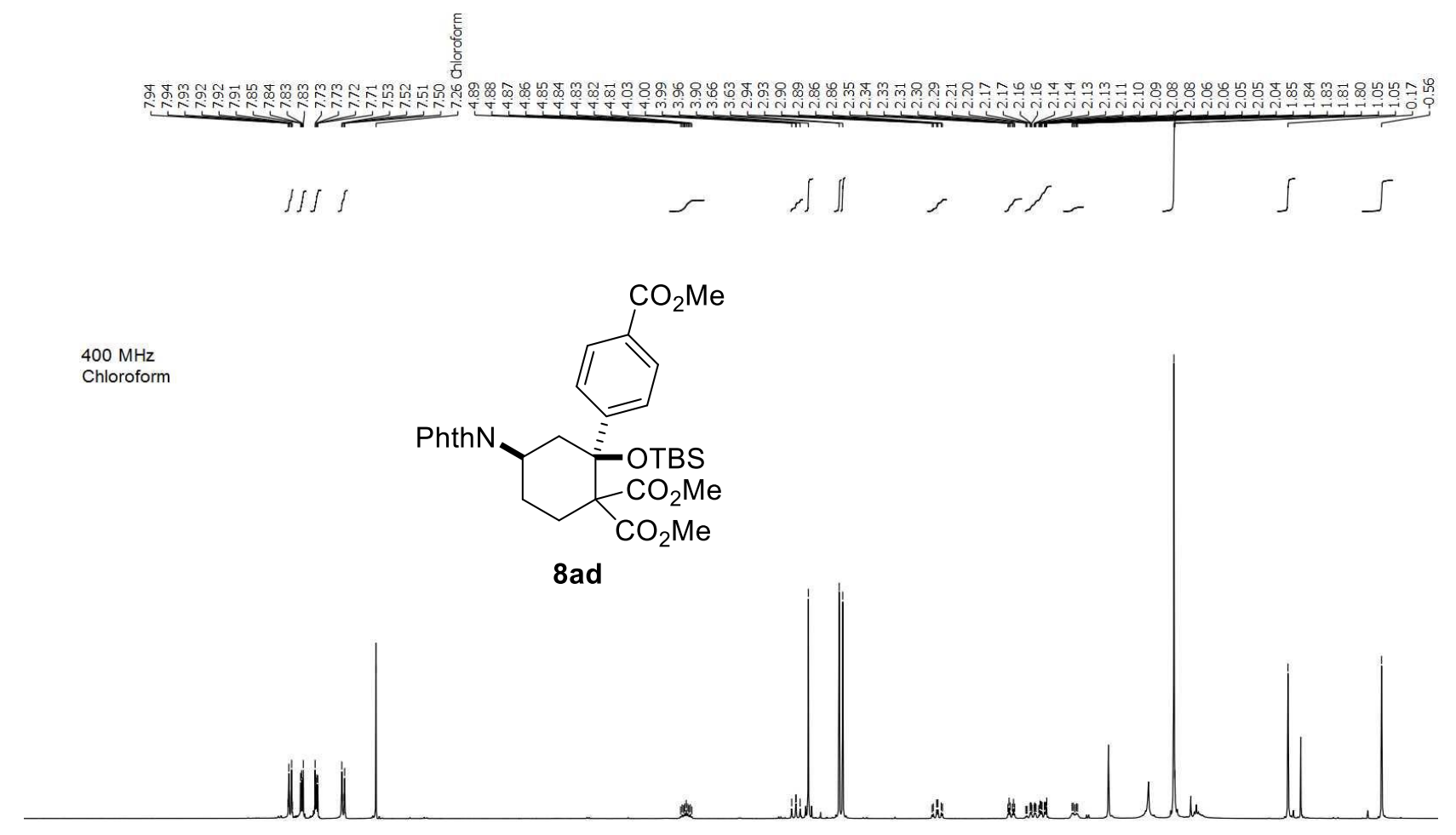

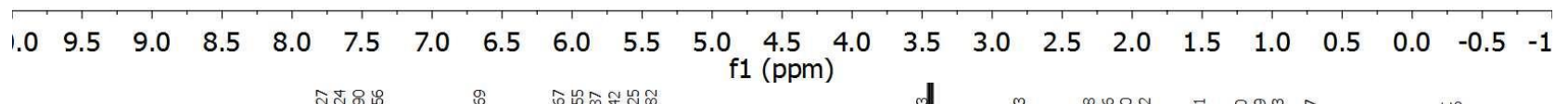

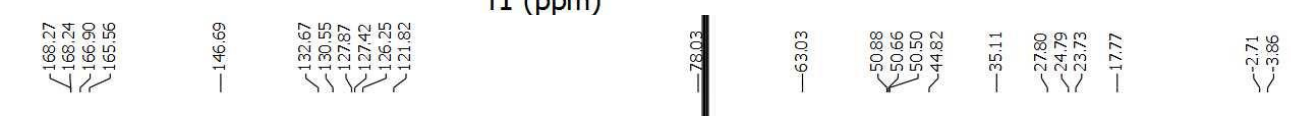

$100 \mathrm{MHz}$

Chloroform

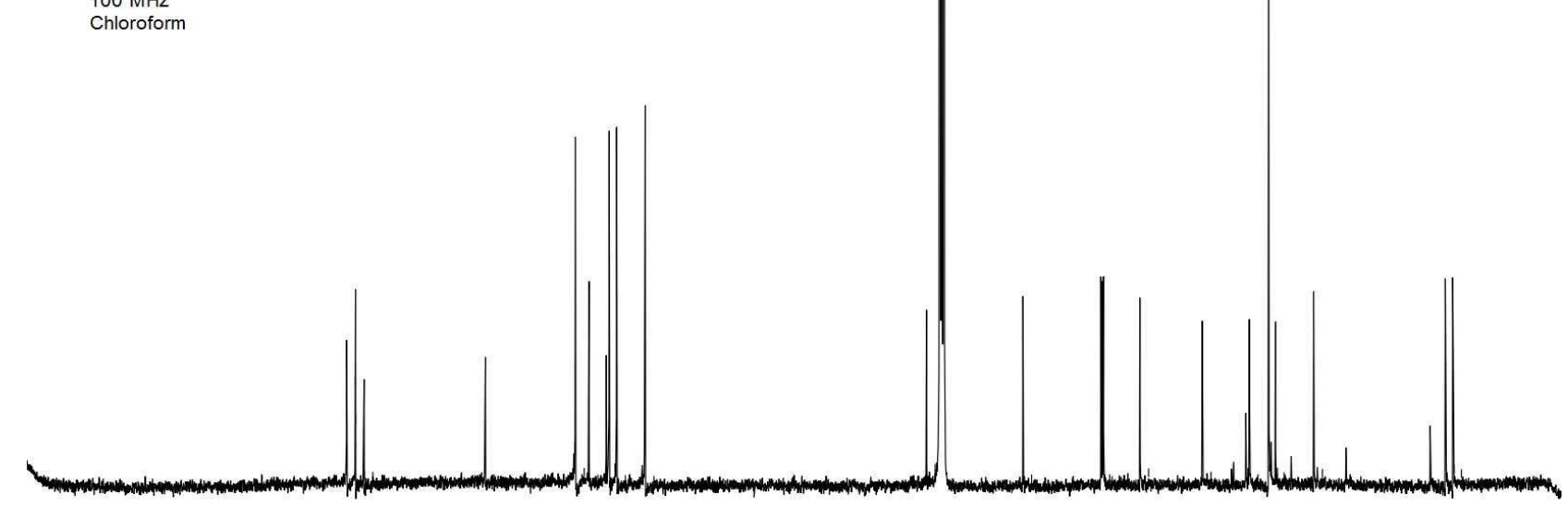

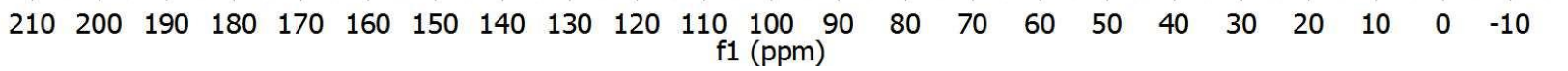



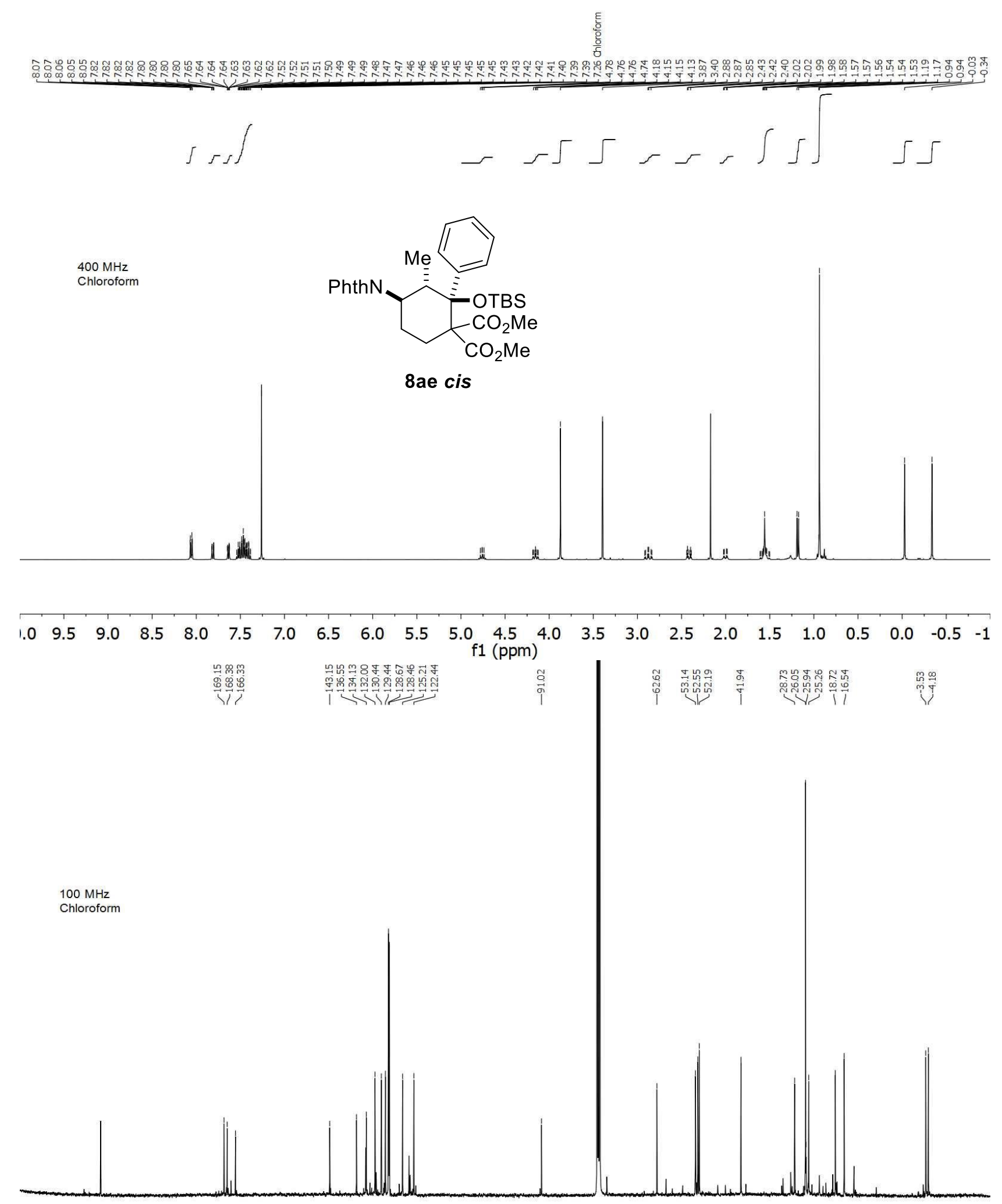

$\begin{array}{lllllllllllllllllllllllll}210 & 200 & 190 & 180 & 170 & 160 & 150 & 140 & 130 & 120 & 110 & 100 & 90 & 80 & 70 & 60 & 50 & 40 & 30 & 20 & 10 & 0 & -10\end{array}$ 


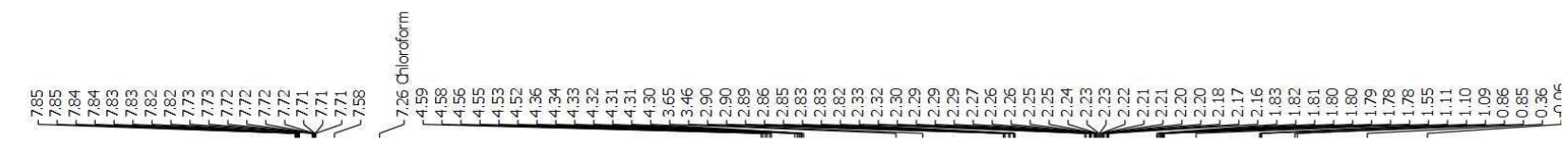

$\iiint$

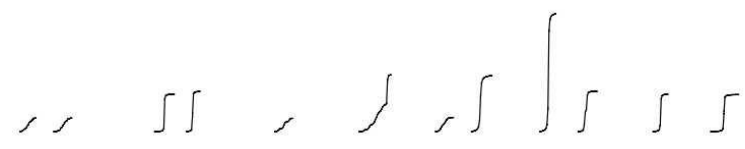

$400 \mathrm{MHz}$

Chloroform

un

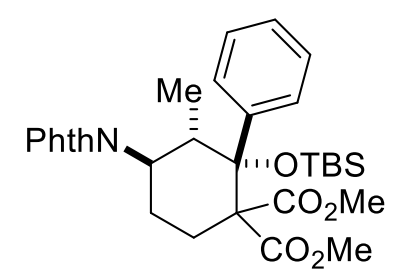

8 ae trans
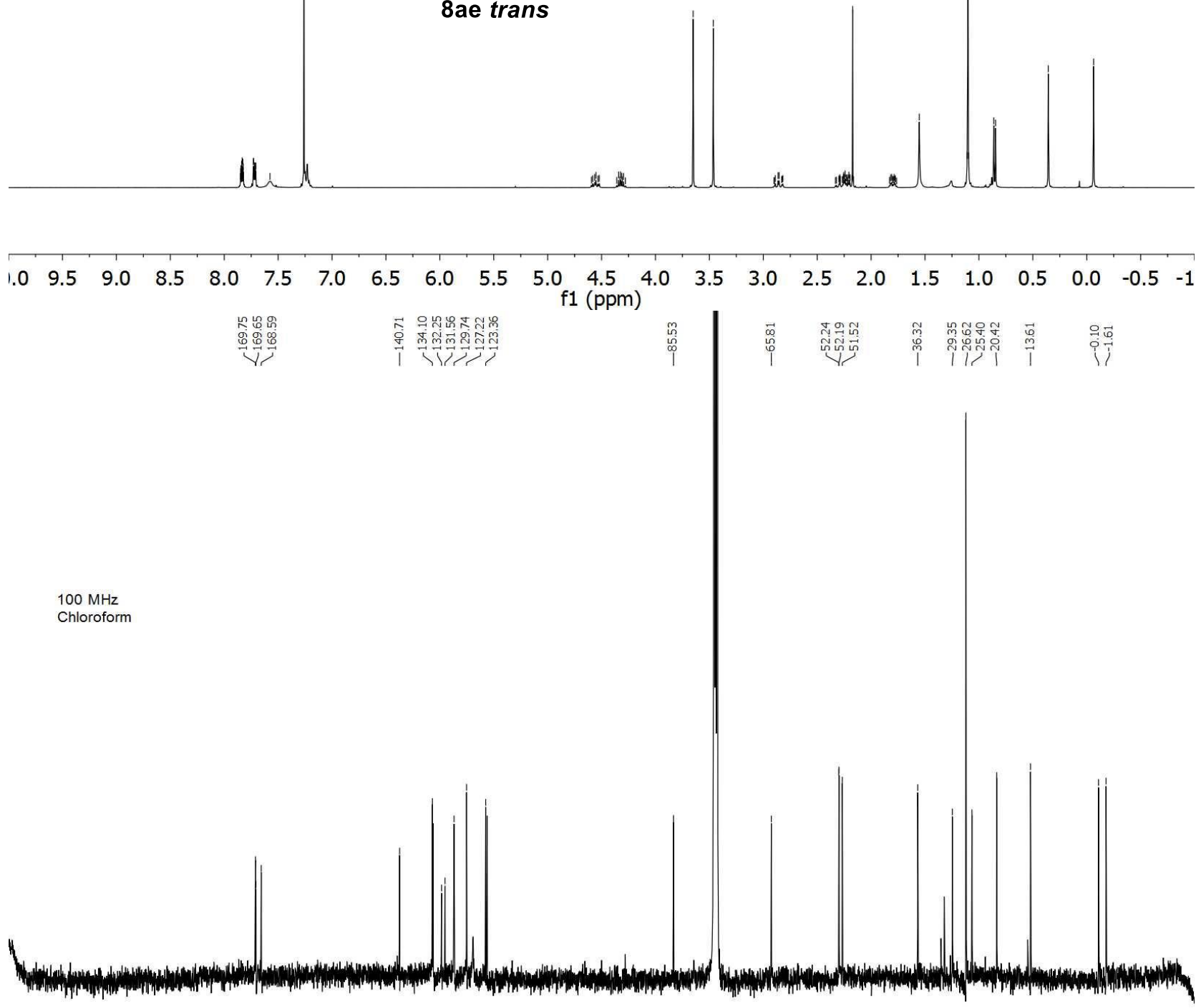

$\begin{array}{lllllllllllllllllllllll}210 & 200 & 190 & 180 & 170 & 160 & 150 & 140 & 130 & 120 & 110 & 100 & 90 & 80 & 70 & 60 & 50 & 40 & 30 & 20 & 10 & 0 & -10\end{array}$ f1 (ppm) 

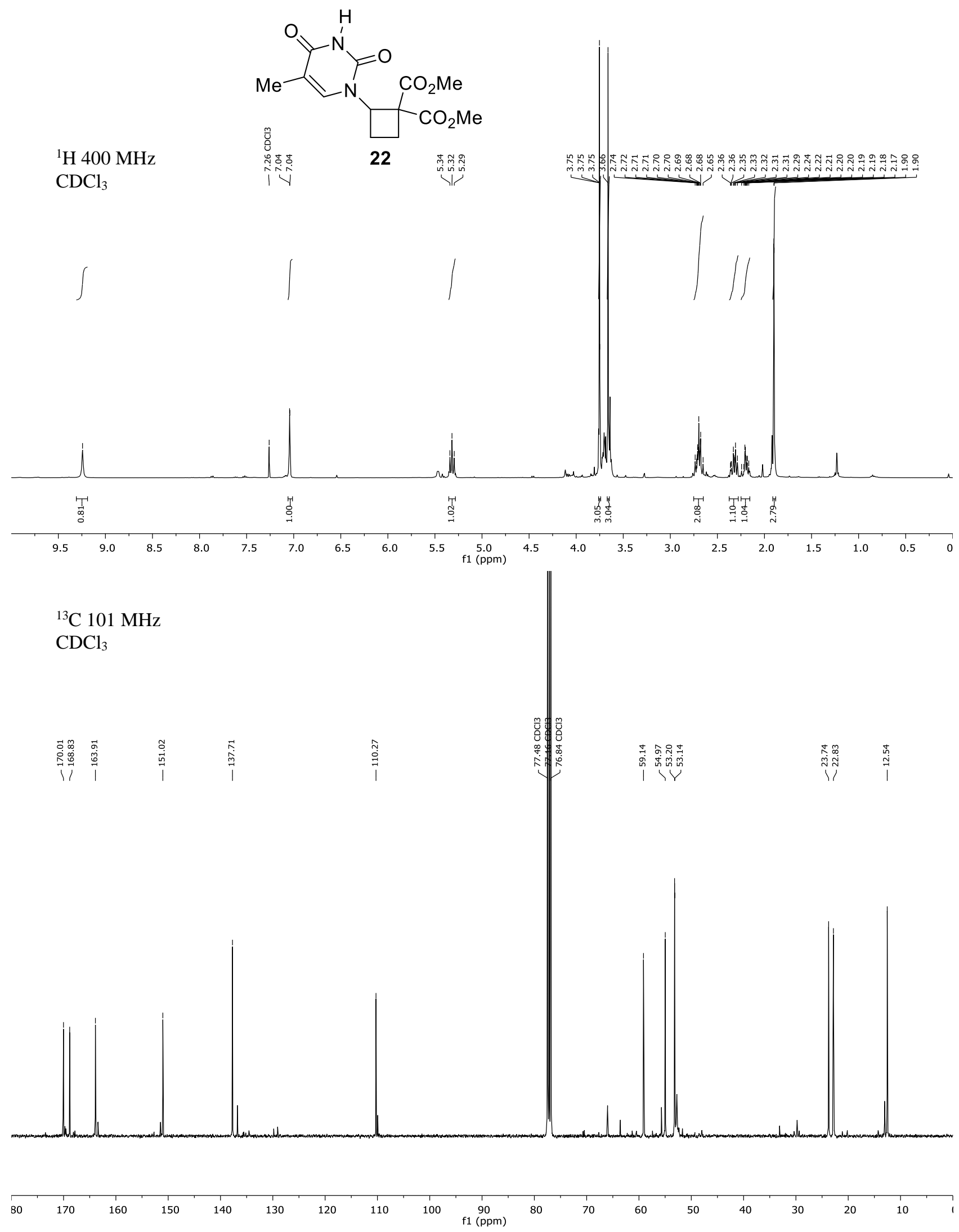

S86 


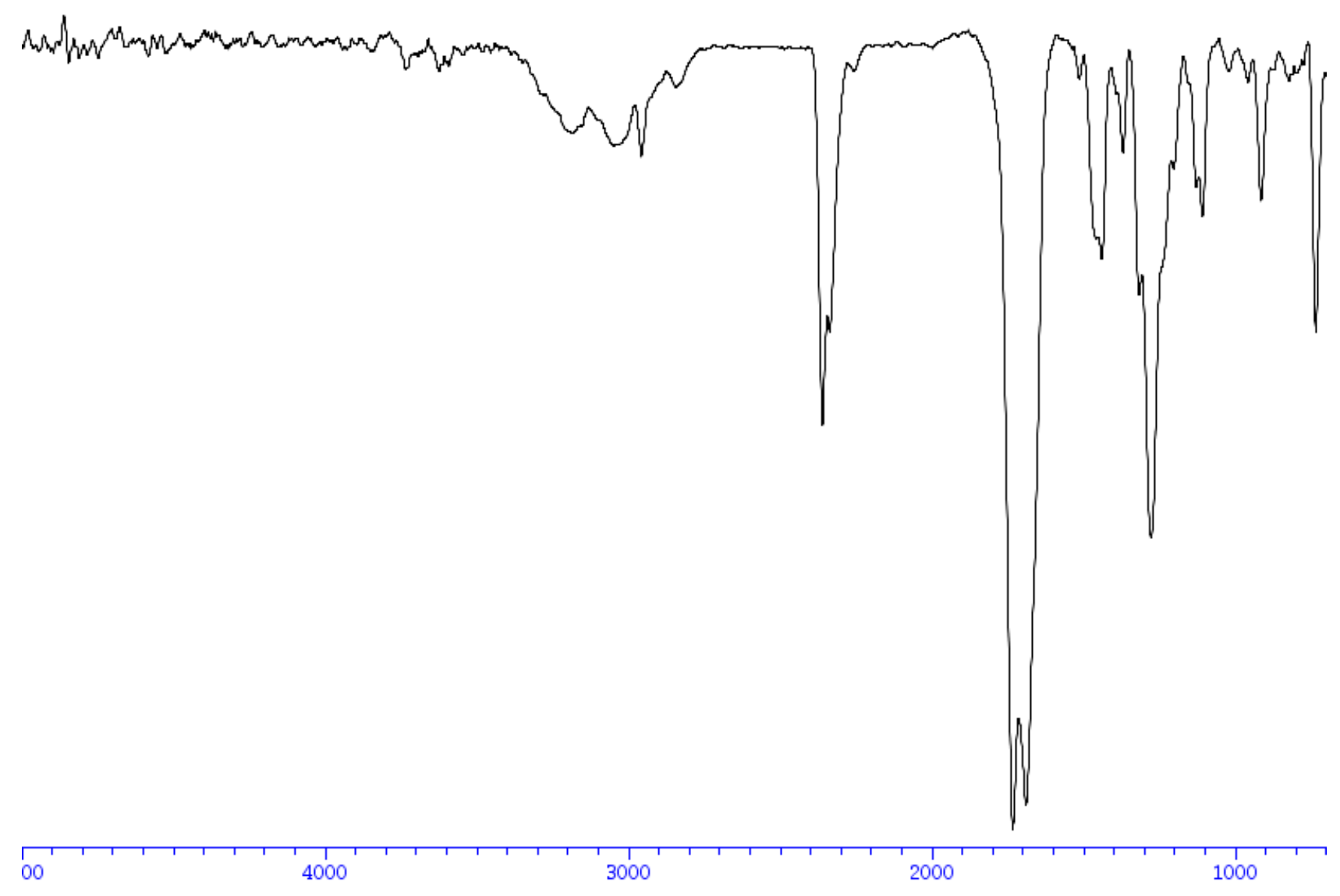



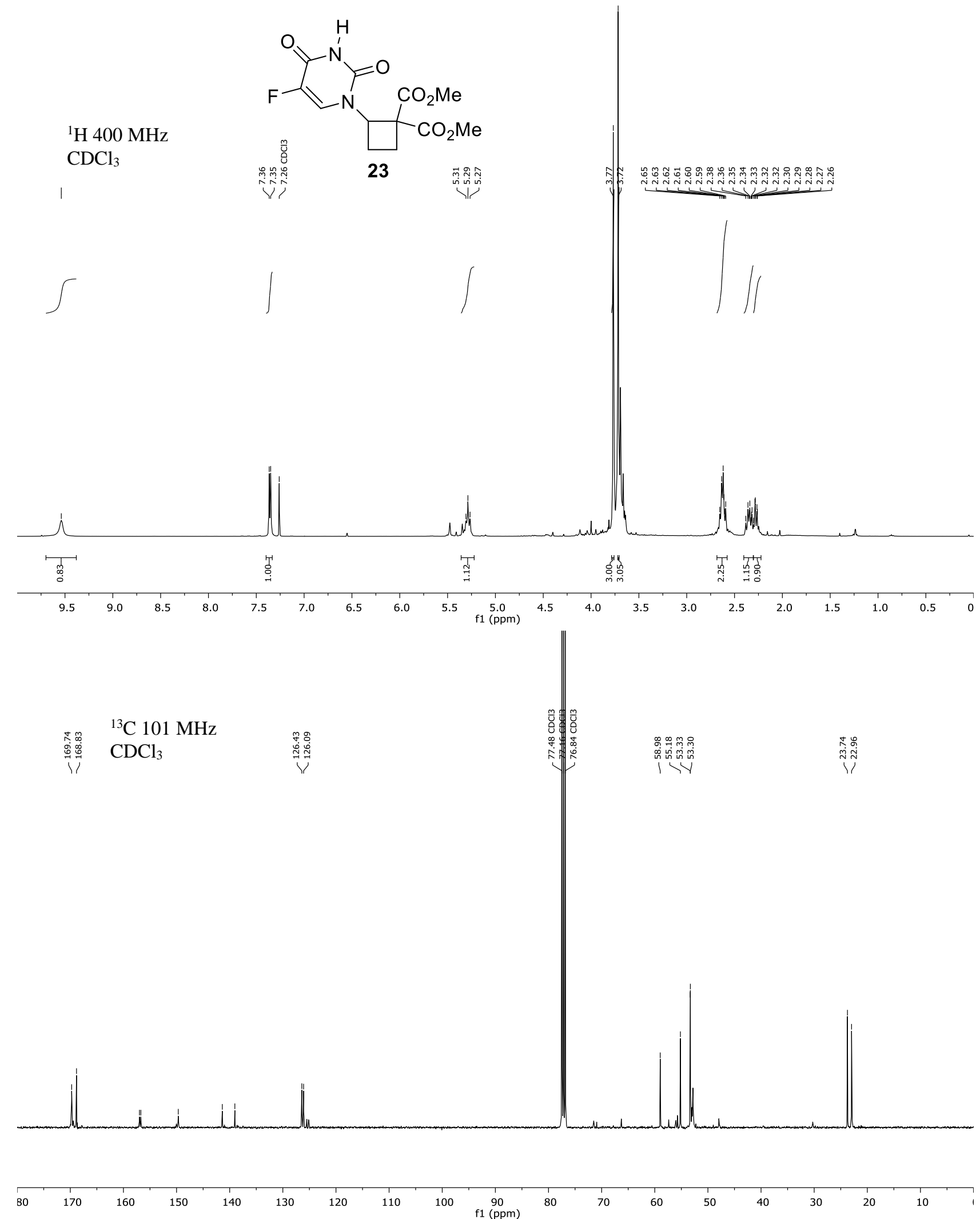


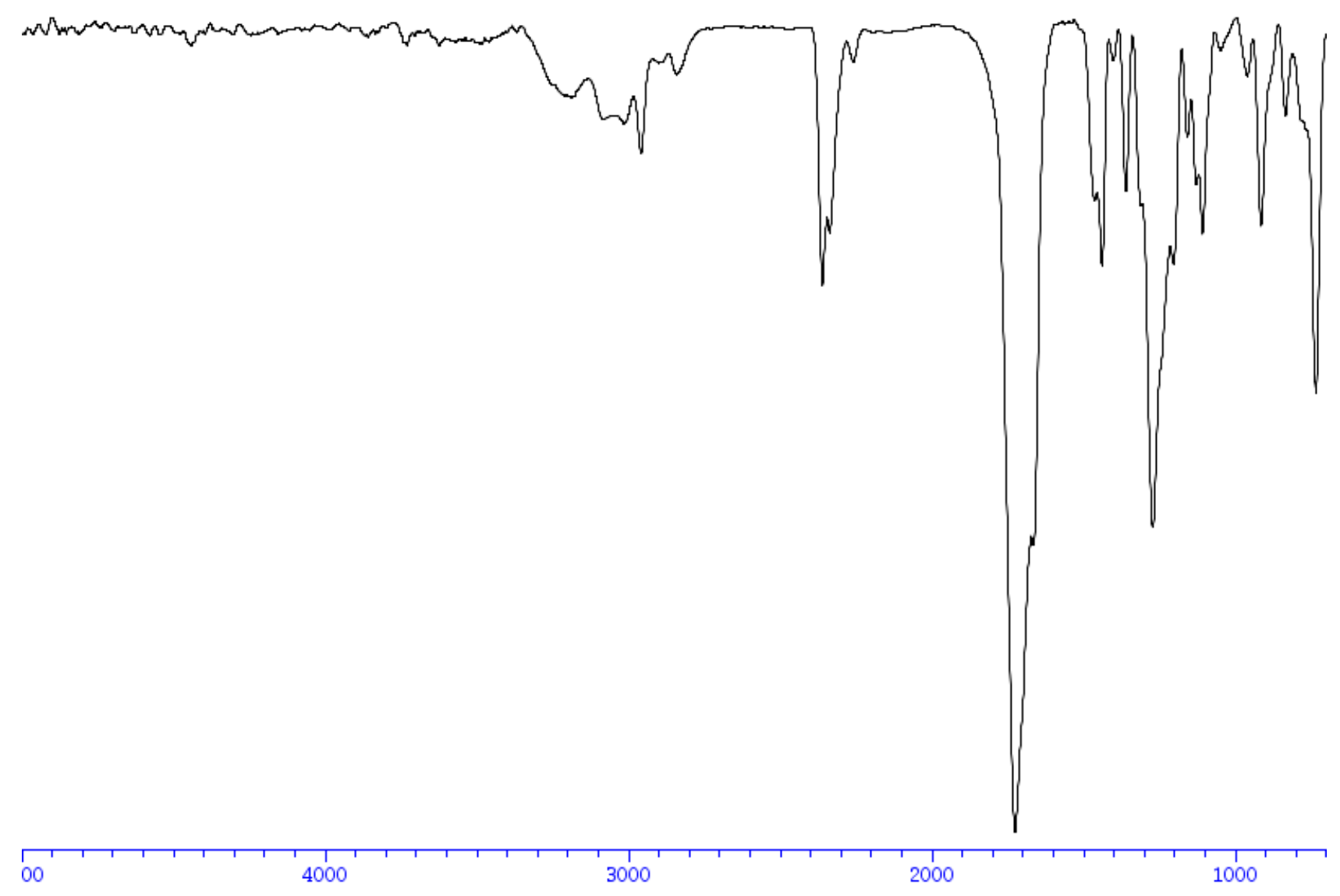

